\title{
Enantioselective Total Synthesis of (+)-Psiguadial B
}

\author{
Lauren M. Chapman, Jordan C. Beck, Linglin Wu, and Sarah E. Reisman* \\ The Warren and Katharine Schlinger Laboratory of Chemistry and Chemical Engineering \\ Division of Chemistry and Chemical Engineering, California Institute of Technology \\ Pasadena, California 91125 \\ reisman@caltech.edu
}

Supporting Information 1 (Experimental Procedures) 
General Procedures. Unless otherwise stated, reactions were performed under a nitrogen atmosphere using freshly dried solvents. Tetrahydrofuran (THF), methylene chloride (DCM), acetonitrile (MeCN), tert-butyl methyl ether (TBME), benzene $(\mathrm{PhH})$, and toluene $(\mathrm{PhMe})$ were dried by passing through activated alumina columns. Triethylamine $\left(\mathrm{Et}_{3} \mathrm{~N}\right), \mathrm{N}, \mathrm{N}$-diisopropylethylamine (DIPEA), and methanol $(\mathrm{MeOH})$ was distilled over calcium hydride prior to use. Unless otherwise stated, chemicals and reagents were used as received. All reactions were monitored by thin-layer chromatography using EMD/Merck silica gel 60 F254 pre-coated plates $(0.25 \mathrm{~mm})$ and were visualized by UV, $p$-anisaldehyde, or 2,4dinitrophenylhydrazine staining. Flash column chromatography was performed either as described by Still et al. ${ }^{1}$ using silica gel (particle size 0.032-0.063) purchased from Silicycle or using pre-packaged RediSep ${ }^{\circledR}$ Rf columns on a CombiFlash Rf system (Teledyne ISCO Inc.). Optical rotations were measured on a Jasco P-2000 polarimeter using a $100 \mathrm{~mm}$ path-length cell at $589 \mathrm{~nm} .{ }^{1} \mathrm{H}$ and ${ }^{13} \mathrm{C}$ NMR spectra were recorded on a Bruker Avance III HD with Prodigy cryoprobe (at $400 \mathrm{MHz}$ and $101 \mathrm{MHz}$ respectively), a Varian $400 \mathrm{MR}$ (at $400 \mathrm{MHz}$ and $101 \mathrm{MHz}$, respectively), a Varian Inova 500 (at $500 \mathrm{MHz}$ and 126 $\mathrm{MHz}$, respectively), or a Varian Inova 600 (at $600 \mathrm{MHz}$ and $150 \mathrm{MHz}$, respectively), and are reported relative to internal $\mathrm{CHCl}_{3}\left({ }^{1} \mathrm{H}, \delta=7.26\right)$, and $\mathrm{CDCl}_{3}\left({ }^{13} \mathrm{C}, \delta=77.1\right)$ or $\mathrm{C}_{6} \mathrm{H}_{5}\left({ }^{1} \mathrm{H}, \delta=7.16\right)$, and $\mathrm{C}_{6} \mathrm{D}_{6}\left({ }^{13} \mathrm{C}\right.$, $\delta=128$ ). Data for ${ }^{1} \mathrm{H}$ NMR spectra are reported as follows: chemical shift ( $\delta \mathrm{ppm}$ ) (multiplicity, coupling constant $(\mathrm{Hz})$, integration). Multiplicity and qualifier abbreviations are as follows: $\mathrm{s}=$ singlet, $\mathrm{d}=$ doublet, $\mathrm{t}=$ triplet, $\mathrm{q}=$ quartet, $\mathrm{m}=$ multiplet, $\mathrm{br}=$ broad, $\mathrm{app}=$ apparent. IR spectra were recorded on a Perkin Elmer Paragon 1000 spectrometer and are reported in frequency of absorption $\left(\mathrm{cm}^{-1}\right)$. HRMS were acquired using an Agilent 6200 Series TOF with an Agilent G1978A Multimode source in electrospray ionization (ESI), atmospheric pressure chemical ionization (APCI), or mixed (MM) ionization mode, or obtained from the Caltech Mass Spectral Facility in fast-atom bombardment mode (FAB). Analytical SFC was performed with a Mettler SFC supercritical $\mathrm{CO}_{2}$ analytical chromatography system with a Chiralcel AD-H column $(4.6 \mathrm{~mm} \times 25 \mathrm{~cm})$. 


\section{Large-scale preparation of enantioenriched cyclobutane 5.}

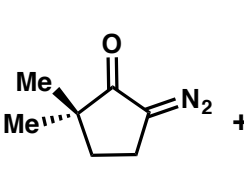

7<smiles>Nc1cccc2cccnc12</smiles>

8 $\frac{\text { (+)-cinchonine }(10 \mathrm{~mol} \%)}{h v(254 \mathrm{~nm}) \mathrm{THF}, \mathrm{rt}}$

$(62 \%$ yield, $79 \%$ ee)

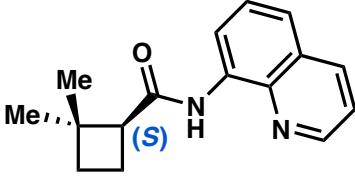

5

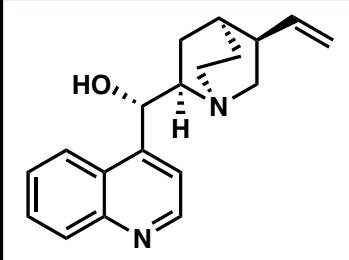

(+)-cinchonine (C4)

To a flame-dried, $1 \mathrm{~L}$ quartz flask was added 8-aminoquinoline (8) (12.9 g, $89.5 \mathrm{mmol}, 3.00$ equiv) and (+)-cinchonine (C4) $(879 \mathrm{mg}, 2.99 \mathrm{mmol}, 0.100$ equiv). The flask was evacuated and backfilled with $\mathrm{N}_{2}$ three times and dry THF $(600 \mathrm{~mL})$ was then added via cannula. Diazoketone $7^{2}(4.12$ $\mathrm{g}, 29.8 \mathrm{mmol}, 1.00$ equiv) was added last via syringe and the reaction was irradiated with stirring using a Honeywell $254 \mathrm{~nm}$ lamp at room temperature. Reaction progress was monitored by TLC (72-168 hours are typically required for complete conversion on this scale, and rotation of the flask every day provided faster conversion). ${ }^{3}$ Upon completion, the reaction mixture was concentrated in vacuo, the solids (C4) were taken up in DCM, and the suspension filtered. The filter cake was washed with DCM three times and the filtrate was concentrated in vacuo to give a crude residue that was purified by silica gel flash chromatography (isocratic: 6\% EtOAc/hexane) to provide 5 (4.69 g, 62\%) as a pale-yellow solid. The enantiomeric excess was determined to be $79 \%$ by chiral SFC analysis (AD-H, $2.5 \mathrm{~mL} / \mathrm{min}, 20 \% \mathrm{IPA}$ in $\mathrm{CO}_{2}, \lambda$ $=254 \mathrm{~nm}): \mathrm{t}_{R}$ (major) $=4.23 \mathrm{~min}, \mathrm{t}_{R}($ minor $)=5.64 \mathrm{~min}$. $[\alpha]_{D}^{25.0}=-66.0^{\circ}\left(\mathrm{c}=0.560, \mathrm{CHCl}_{3}\right)$.

\section{Recrystallization procedure:}

Enantioenriched cyclobutane $\mathbf{5}$ was dissolved in a minimal amount of DCM in a $100 \mathrm{~mL}$ round-bottom flask. An equal amount of hexanes was carefully layered on top of the DCM to form a biphasic mixture. The layers were allowed to diffuse overnight to provide $\mathbf{5}$ as white needles. The supernatant was concentrated under reduced pressure and this process was repeated again to provide additional $\mathbf{5}$ (3.50 g total, $83 \%$ recovery of theoretical total of the desired

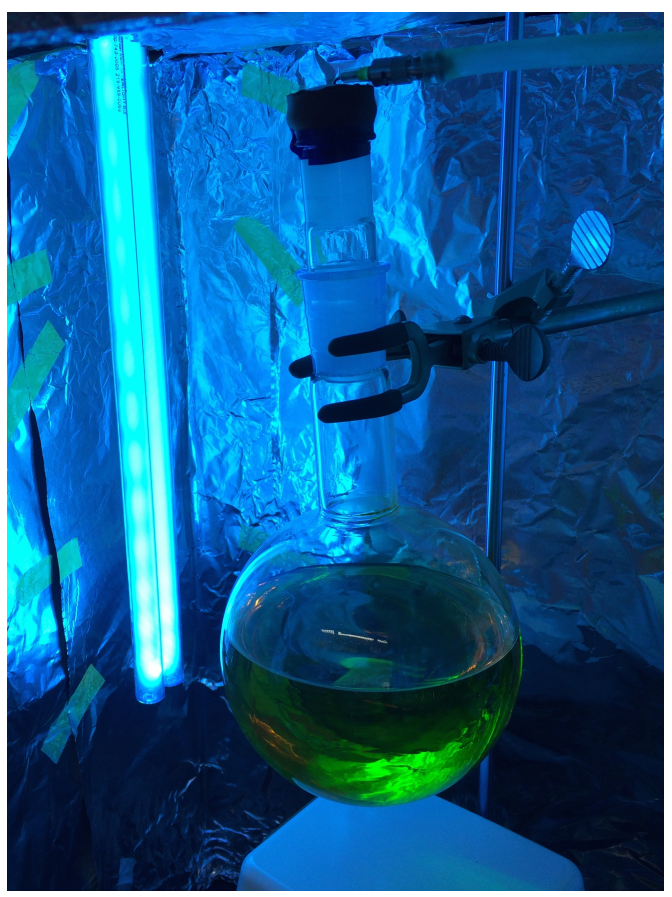
enantiomer, $46 \%$ overall from 7): $[\alpha]_{D}^{25.0}=-109^{\circ}\left(\mathrm{c}=0.720, \mathrm{CHCl}_{3}\right)$. 
${ }^{1}$ H NMR $\left(400 \mathrm{MHz}, \mathrm{CDCl}_{3}\right) \delta 9.68(\mathrm{~s}, 1 \mathrm{H}), 8.80(\mathrm{t}, J=1.8 \mathrm{~Hz}, 1 \mathrm{H}), 8.79(\mathrm{dd}, J=13.6,1.6 \mathrm{~Hz}, 1 \mathrm{H}), 8.15$ $(\mathrm{dd}, J=8.3,1.7 \mathrm{~Hz}, 1 \mathrm{H}), 7.52$ (q, $J=8.2,7.5 \mathrm{~Hz}, 1 \mathrm{H}), 7.48(\mathrm{dd}, J=8.3,1.6 \mathrm{~Hz}, 1 \mathrm{H}), 7.45(\mathrm{dd}, J=8.3$, $4.2 \mathrm{~Hz}, 1 \mathrm{H}), 3.07$ (ddd, $J=9.1,8.2,0.9 \mathrm{~Hz}, 1 \mathrm{H}), 2.48$ (dq, $J=11.4,9.4 \mathrm{~Hz}, 1 \mathrm{H}$ ), 2.06 (dtd, $J=11.6,8.6$, $3.3 \mathrm{~Hz}, 1 \mathrm{H}), 1.85$ (dt, $J=10.8,9.1 \mathrm{~Hz}, 1 \mathrm{H}), 1.74(\mathrm{dddd}, J=10.7,9.5,3.3,0.9 \mathrm{~Hz}, 1 \mathrm{H}), 1.39$ (s, $3 \mathrm{H}), 1.14$ $(\mathrm{s}, 3 \mathrm{H})$.

${ }^{13}$ C NMR $\delta 171.8,148.3,138.6,136.4,134.7,128.1,127.6,121.7,121.3,116.4,51.0,40.4,32.3,30.9$, 23.4, 17.4 .

FTIR $(\mathrm{NaCl}$, thin film) 3353, 3047, 2952, 2861, 1685, 1595, 1577, 1526, 1485, 1460, 1424, 1385, 1324, $1261,1239,1187,1169,1153,825,791,756 \mathrm{~cm}^{-1}$

HRMS (MM) calc'd for $\mathrm{C}_{16} \mathrm{H}_{19} \mathrm{~N}_{2} \mathrm{O}[\mathrm{M}+\mathrm{H}]^{+} 255.1492$, found 255.1501 .

\section{SFC data for racemic 5:}

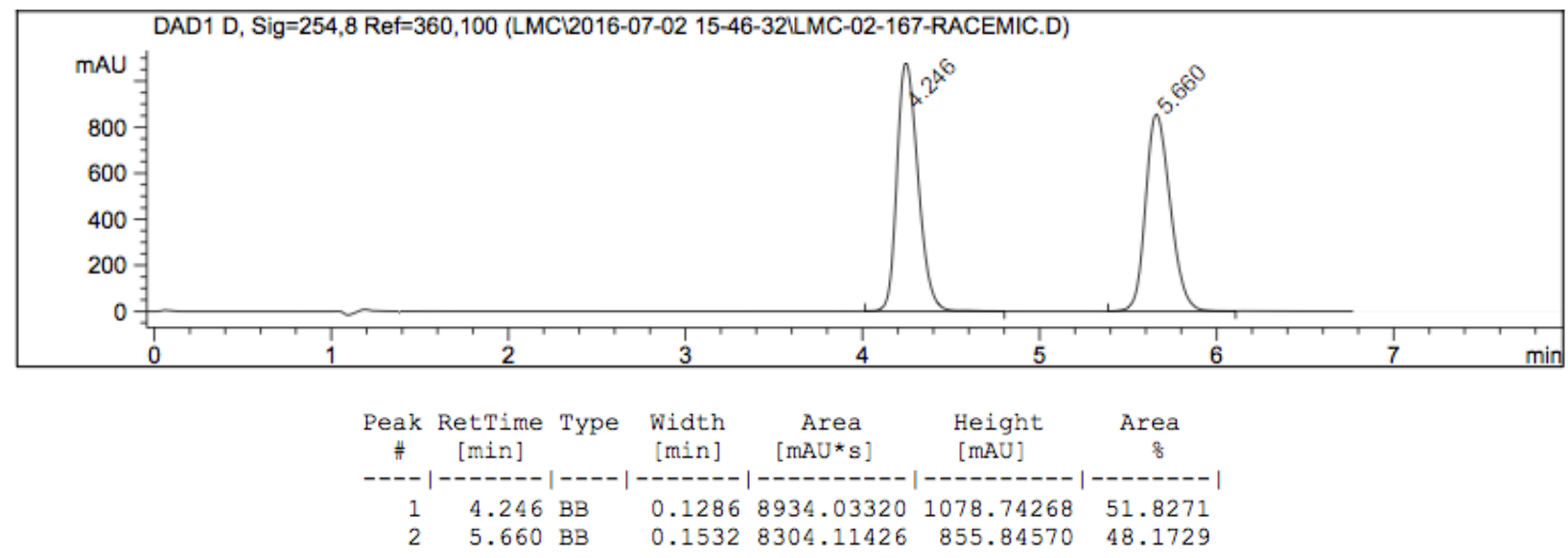

Enantioenriched 5 isolated directly from reaction:

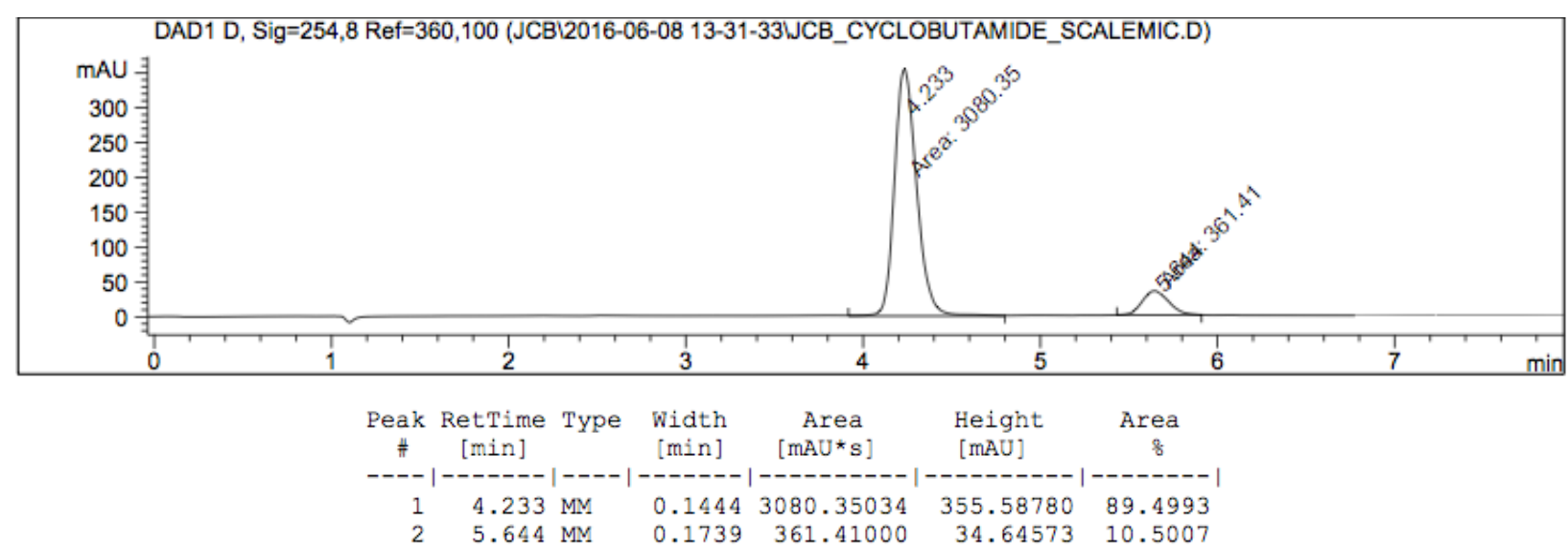


Enantiopure 5 after a single recrystallization:

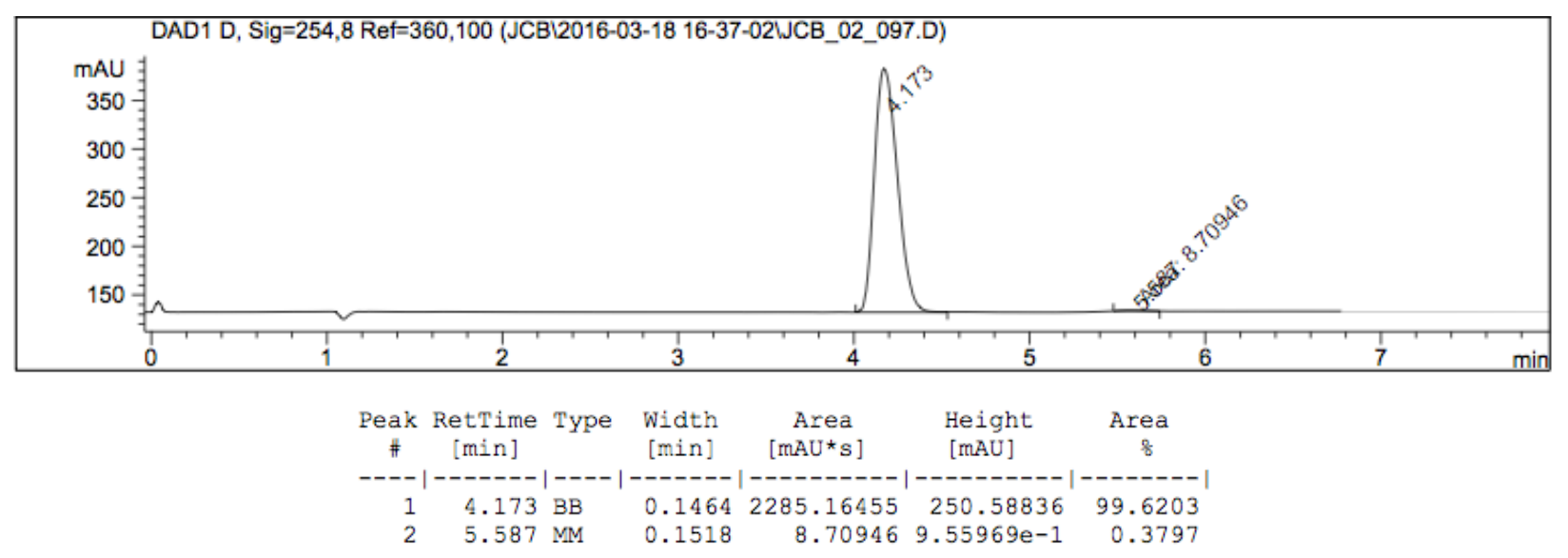

\section{Small-scale screening protocol for cyclobutane 5.}

Inside a $\mathrm{N}_{2}$-filled glovebox, six oven-dried quartz tubes were charged with aminoquinoline (14.4 $\mathrm{mg}, 0.100 \mathrm{mmol}, 1.00$ equiv) and catalyst (20-50 mol \%). Diazoketone 7 (13.8 $\mathrm{mg}, 0.100 \mathrm{mmol})$ was then added to each as a solution in $1.00 \mathrm{~mL}$ THF and the reactions fitted with a 19/38 rubber septum around the outside of each tube and sealed with electrical tape. The reactions were brought out of the glovebox and placed in a bottomless test tube rack in front of a Honeywell $254 \mathrm{~nm}$ lamp. The reactions were irradiated with stirring at room temperature for 24 hours. The reactions were then concentrated in vacuo and the crude reactions mixtures either analyzed by ${ }^{1} \mathrm{H}$ NMR with an added internal standard, or purified by silica gel flash chromatography (isocratic: $6 \%$ EtOAc/hexane) to provide $\mathbf{5}$ in varying yields and enantiopurities.

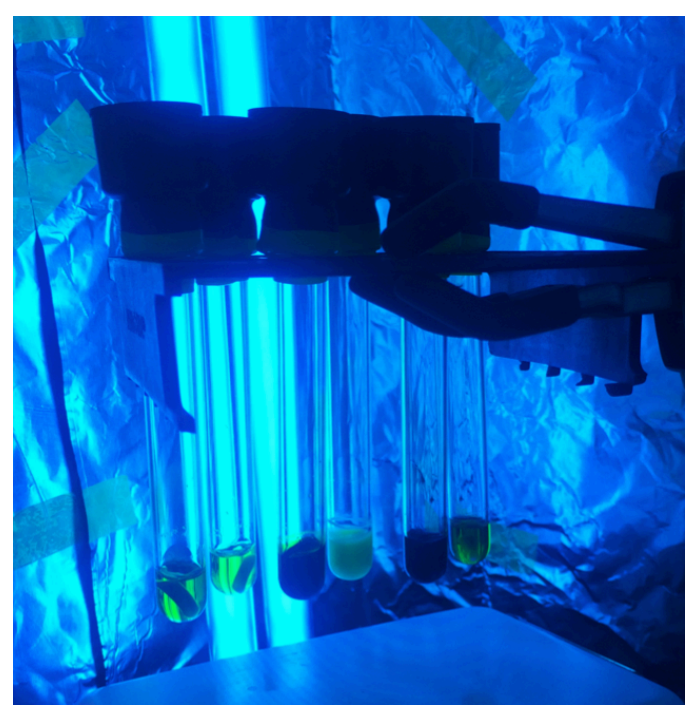


Additional experimental results for optimization of asymmetric Wolff rearrangement:

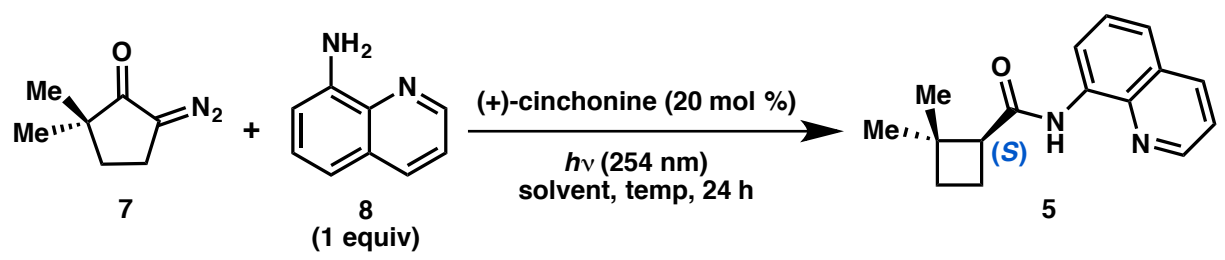

\begin{tabular}{ccccccc}
\hline entry & solvent & temp $^{\circ} \mathrm{C}$ & vessel & atm & $\%$ yielda $^{a}$ & ee $^{b}$ \\
\hline 1 & THF & 25 & quartz & $\mathrm{N}_{2}$ & 63 & 79 \\
2 & DMF & 25 & quartz & $\mathrm{N}_{2}$ & 76 & 50 \\
3 & DCM & 25 & quartz & $\mathrm{N}_{2}$ & 62 & 47 \\
4 & toluene & 25 & quartz & $\mathrm{N}_{2}$ & 65 & 59 \\
5 & dioxane & 25 & quartz & $\mathrm{N}_{2}$ & 69 & 79 \\
6 & MeCN & 25 & quartz & $\mathrm{N}_{2}$ & 43 & 74 \\
7 & THF & 0 & quartz & $\mathrm{N}_{2}$ & 28 & 79 \\
8 & THF & 25 & quartz & air & 18 & 75 \\
9 & THF & 25 & quartz & $\mathrm{CO}$ & 33 & 69 \\
10 & THF & 25 & vycor & $\mathrm{N}_{2}$ & 29 & 79 \\
11 & THF & 25 & pyrex & $\mathrm{N}_{2}$ & 20 & 75 \\
\hline
\end{tabular}

a Determined by ${ }^{1} \mathrm{H}$ NMR via integration relative to an added internal standard. ${ }^{b}$ Determined by SFC using a chiral stationary phase.

Preparation of cis-cyclobutane 10.
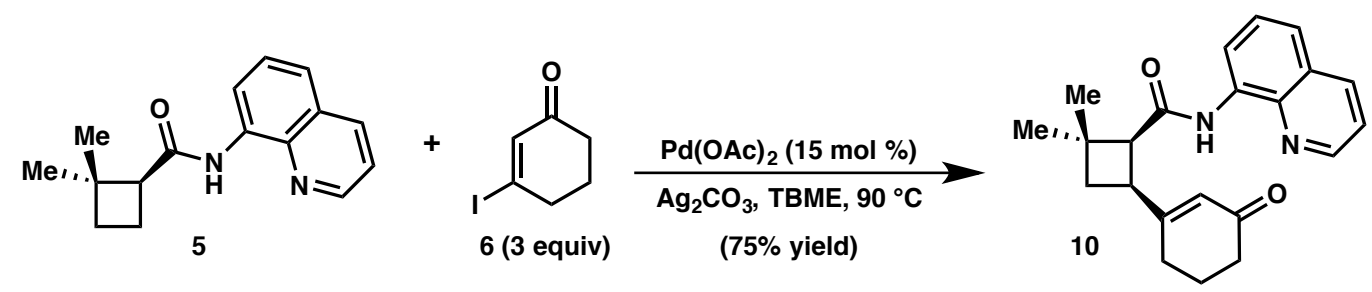

To a flame-dried $150 \mathrm{~mL}$ pressure vessel were added cyclobutane 5 (2.87 g, $11.3 \mathrm{mmol})$, vinyl iodide $6^{4}$ (7.50 g, $33.8 \mathrm{mmol}, 3.00$ equiv), $\mathrm{Pd}(\mathrm{OAc})_{2}$ (379 mg, $1.69 \mathrm{mmol}, 0.150$ equiv), and $\mathrm{Ag}_{2} \mathrm{CO}_{3}$ ( $3.11 \mathrm{~g}, 11.3 \mathrm{mmol}, 1.00$ equiv). The reagents were suspended in TBME $(56.0 \mathrm{~mL})$ and the vessel sealed under ambient conditions. The reaction was heated to $90{ }^{\circ} \mathrm{C}$ for 16 hours, then cooled to room temperature and filtered over a pad of celite. The filtrate was concentrated directly onto celite and purified by silica gel flash chromatography (20-40\% EtOAc/hexane) to afford cis-cyclobutane 10 (2.95 g, 75\%) as a pale yellow foam: $[\alpha]_{D}^{25.0}=+84.4^{\circ}\left(\mathrm{c}=0.350, \mathrm{CHCl}_{3}\right)$.

${ }^{1}$ H NMR $\left(500 \mathrm{MHz}, \mathrm{CDCl}_{3}\right) \delta 9.73(\mathrm{~s}, 1 \mathrm{H}), 8.78(\mathrm{dd}, J=12.4,2.1 \mathrm{~Hz}, 1 \mathrm{H}), 8.78(\mathrm{t}, J=1.8 \mathrm{~Hz}, 1 \mathrm{H}), 8.16$ $(\mathrm{dd}, J=8.3,1.7 \mathrm{~Hz}, 1 \mathrm{H}), 7.51(\mathrm{dd}, J=8.3,5.0 \mathrm{~Hz}, 1 \mathrm{H}), 7.50(\mathrm{~d}, J=0.9 \mathrm{~Hz}, 1 \mathrm{H}), 7.45(\mathrm{dd}, J=8.3,4.2$ $\mathrm{Hz}, 1 \mathrm{H}), 6.00$ (q, $J=1.6 \mathrm{~Hz}, 1 \mathrm{H}), 3.45$ (ddddd, $J=10.8,8.5,7.6,2.1,1.0 \mathrm{~Hz}, 1 \mathrm{H}$ ), 3.27 (ddd, $J=8.8,2.8$, $0.8 \mathrm{~Hz}, 1 \mathrm{H}), 2.48(\mathrm{t}, J=10.8 \mathrm{~Hz}, 1 \mathrm{H}), 2.31(\mathrm{ddd}, J=7.5,5.7,3.5 \mathrm{~Hz}, 2 \mathrm{H}), 2.20(\mathrm{qd}, J=6.0,5.5,1.1 \mathrm{~Hz}$, 2H), 2.01 (ddd, $J=11.0,8.3,2.8 \mathrm{~Hz}, 1 \mathrm{H}), 1.95-1.84$ (m, 2H), $1.46(\mathrm{~s}, 3 \mathrm{H}), 1.13$ (s, 3H). 
${ }^{13}$ C NMR $\left(126 \mathrm{MHz}, \mathrm{CDCl}_{3}\right) \delta 199.5,170.2,166.5,148.3,138.4,136.3,134.4,127.9,127.4,124.9$, $121.6,121.5,116.5,56.9,37.5,37.5,36.8,35.7,29.9,27.8,24.9,22.6$.

FTIR ( $\mathrm{NaCl}$, thin film) 3348, 2929, 2865, 1662, 1623, 1595, 1576, 1522, 1485, 1424, 1386, 1347, 1322 , $1258,1191,1165,1132,827,793 \mathrm{~cm}^{-1}$

HRMS (MM) calc'd for $\mathrm{C}_{22} \mathrm{H}_{25} \mathrm{~N}_{2} \mathrm{O}_{2}[\mathrm{M}+\mathrm{H}]^{+} 349.1911$, found 349.1910.

Preparation of trans-cyclobutane 11 and spirolactam S1.
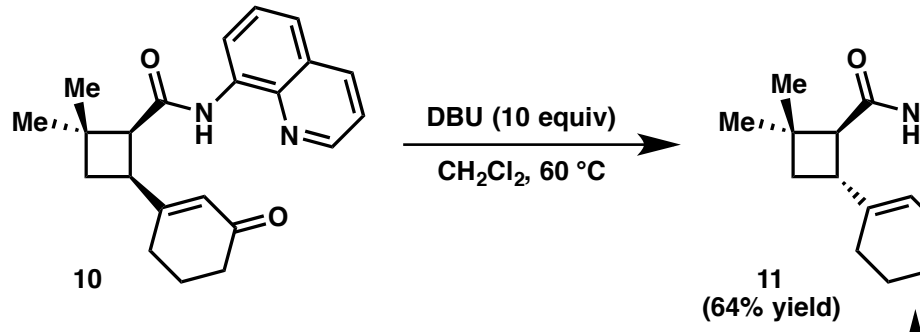

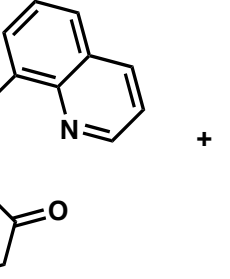

DBU, $\mathrm{CH}_{2} \mathrm{Cl}_{2}, 60^{\circ} \mathrm{C}$

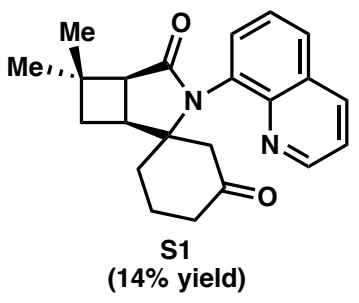

$(14 \%$ yield)

To a $150 \mathrm{~mL}$ pressure vessel were added cis-cyclobutane $10(2.74 \mathrm{~g}, 7.86 \mathrm{mmol})$ and wet DCM $(27.5 \mathrm{~mL})$. The colorless solution was treated with DBU $(11.7 \mathrm{~mL}, 78.6 \mathrm{mmol}, 10.0$ equiv) and a bright yellow color was observed immediately. The vessel was sealed under ambient conditions and heated to 60 ${ }^{\circ} \mathrm{C}$ for 20 hours. The reaction mixture was diluted with $100 \mathrm{~mL}$ of water and $100 \mathrm{~mL}$ of DCM. The layers were separated, and the aqueous layer was extracted with DCM (3 x $50 \mathrm{~mL})$. The combined organic layers were dried over $\mathrm{Na}_{2} \mathrm{SO}_{4}$, filtered, and concentrated in vacuo. The crude residue was purified by silica gel flash chromatography (isocratic: 40\% EtOAc/hexane until 11 eluted completely, then 10\% $\mathrm{MeOH} / \mathrm{DCM})$ to afford $\mathbf{1 1}$ (1.74 g, 64\% yield) and $\mathbf{S 1}$ (367 mg, 14\% yield), each as a pale yellow solid.

Data for trans-cyclobutane 11: $[\alpha]_{D}^{25.0}=-129.0^{\circ}\left(\mathrm{c}=1.43, \mathrm{CHCl}_{3}\right)$.

${ }^{1}$ H NMR $\left(500 \mathrm{MHz}, \mathrm{CDCl}_{3}\right) \delta 9.68(\mathrm{~s}, 1 \mathrm{H}), 8.79(\mathrm{dd}, J=4.2,1.7 \mathrm{~Hz}, 1 \mathrm{H}), 8.73(\mathrm{dd}, J=7.2,1.8 \mathrm{~Hz}, 1 \mathrm{H})$, $8.15(\mathrm{dd}, J=8.3,1.7 \mathrm{~Hz}, 1 \mathrm{H}), 7.52(\mathrm{dd}, J=8.3,7.2 \mathrm{~Hz}, 1 \mathrm{H}), 7.49(\mathrm{dd}, J=8.3,1.8 \mathrm{~Hz}, 1 \mathrm{H}), 7.44(\mathrm{dd}, J=$ $8.3,4.2 \mathrm{~Hz}, 1 \mathrm{H}), 5.92(\mathrm{q}, J=1.5 \mathrm{~Hz}, 1 \mathrm{H}), 3.58(\mathrm{ddq}, J=18.5,8.7,1.6,0.8,0.8 \mathrm{~Hz}, 1 \mathrm{H}), 2.97(\mathrm{dd}, J=9.8$, $0.7 \mathrm{~Hz}, 1 \mathrm{H}), 2.41-2.29(\mathrm{~m}, 4 \mathrm{H}), 2.05-1.92(\mathrm{~m}, 3 \mathrm{H}), 1.85(\mathrm{t}, J=10.4 \mathrm{~Hz}, 1 \mathrm{H}), 1.40(\mathrm{~s}, 3 \mathrm{H}), 1.19(\mathrm{~s}$, $3 \mathrm{H})$.

${ }^{13}$ C NMR $\left(126 \mathrm{MHz}, \mathrm{CDCl}_{3}\right) \delta 199.8,169.6,167.5,148.3,138.3,136.3,134.2,127.9,127.3,123.9$, $121.7,121.5,116.3,55.5,37.5,36.8,36.4,36.3,30.7,27.6,23.1,22.6$.

FTIR ( $\mathrm{NaCl}$, thin film) 3344, 3046, 2952, 2865, 2246, 1669, 1623, 1595, 1577, 1526, 1485, 1461, 1424, $1323,1346,1326,1292,1253,1191,1161,1133,915,884,827,792,757,731 \mathrm{~cm}^{-1}$ 
HRMS (MM) calc'd for $\mathrm{C}_{22} \mathrm{H}_{25} \mathrm{~N}_{2} \mathrm{O}_{2}[\mathrm{M}+\mathrm{H}]^{+} 349.1911$, found 349.1919.

XRCD: A suitable crystal of $\mathrm{C}_{22} \mathrm{H}_{24} \mathrm{~N}_{2} \mathrm{O}_{2}(\mathbf{1 1})$ was selected for analysis. All measurements were made on a Bruker APEX-II CCD with filtered $\mathrm{Cu}-\mathrm{K} \alpha$ radiation at a temperature of $120 \mathrm{~K}$. Using Olex $2,{ }^{5}$ the structure was solved with the ShelXS ${ }^{6}$ structure solution program using Direct Methods and refined with the ShelXL refinement package using Least Squares minimization. The absolute stereochemistry
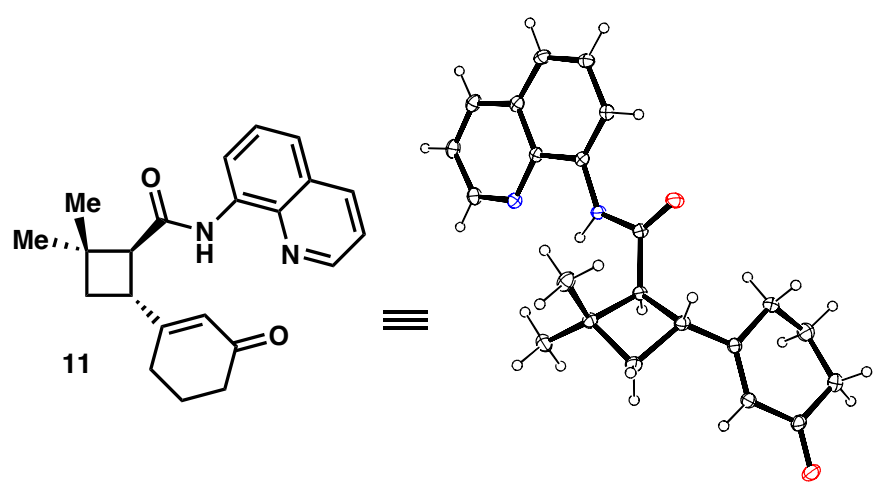
was determined on the basis of the absolute structure parameter, $-0.04(4)$.

Data for spirolactam S1, 2.5:1 mixture of diastereomers: $[\alpha]_{D}^{25.0}=-56.5^{\circ}\left(\mathrm{c}=1.085, \mathrm{CHCl}_{3}\right)$.

${ }^{1}$ H NMR (asterisk denotes minor diast., $\left.400 \mathrm{MHz}, \mathrm{CDCl}_{3}\right) \delta 8.92(\mathrm{dd}, J=4.1,1.8 \mathrm{~Hz}, 1 \mathrm{H}), 8.85^{*}(\mathrm{dd}, J=$ 4.1, $1.8 \mathrm{~Hz}, 1 \mathrm{H}), 8.17(\mathrm{dd}, J=8.3,1.8 \mathrm{~Hz}, 1 \mathrm{H}), 8.13^{*}(\mathrm{dd}, J=8.3,1.8 \mathrm{~Hz}, 1 \mathrm{H}), 7.87$ (dd, $J=8.3,1.5 \mathrm{~Hz}$, 1H), $7.63-7.53(\mathrm{~m}, 2 \mathrm{H}), 7.49(\mathrm{dd}, J=7.2,1.5 \mathrm{~Hz}, 1 \mathrm{H}), 7.42(\mathrm{dd}, J=8.3,4.1 \mathrm{~Hz}, 1 \mathrm{H}), 7.37^{*}(\mathrm{dd}, J=8.3$, $4.1 \mathrm{~Hz}, 1 \mathrm{H}), 3.07 *$ (ddd, $J=7.3,3.2,0.9 \mathrm{~Hz}, 1 \mathrm{H}), 2.93(\mathrm{dd}, J=6.0,3.3 \mathrm{~Hz}, 1 \mathrm{H}), 2.86(\mathrm{~d}, J=13.2 \mathrm{~Hz}$, 1H), $2.83-2.73(\mathrm{~m}, 1 \mathrm{H}), 2.53(\mathrm{dt}, J=13.1,2.4 \mathrm{~Hz}, 1 \mathrm{H}), 2.41-2.33(\mathrm{~m}, 1 \mathrm{H}), 2.27(\mathrm{ddq}, J=15.1,11.3$, $2.1 \mathrm{~Hz}, 1 \mathrm{H}), 2.15-1.94(\mathrm{~m}, 2 \mathrm{H}), 1.94-1.83(\mathrm{~m}, 1 \mathrm{H}), 1.71(\mathrm{dtd}, J=13.2,8.6,7.6,3.0 \mathrm{~Hz}, 1 \mathrm{H}), 1.52-$ $1.38^{*}(\mathrm{~m}, 1 \mathrm{H}), 1.35(\mathrm{~s}, 3 \mathrm{H}), 1.34^{*}(\mathrm{~s}, 3 \mathrm{H}), 1.31(\mathrm{~s}, 3 \mathrm{H}), 1.14^{*}(\mathrm{~s}, 3 \mathrm{H}), 1.03(\mathrm{td}, J=13.7,4.0 \mathrm{~Hz}, 1 \mathrm{H})$.

${ }^{13}$ C NMR (major diastereomer, $101 \mathrm{MHz}, \mathrm{CDCl}_{3}$ ) $\delta$ 209.2, 175.5, 150.7, 146.3, 136.1, 134.2, 130.7, 129.6, 129.1, 126.2, 121.9, 69.2, 53.2, 49.9, 40.7, 35.8, 34.8, 34.1, 30.2, 29.0, 25.6, 20.1.

${ }^{13} \mathbf{C}$ NMR (minor diastereomer, $101 \mathrm{MHz}, \mathrm{CDCl}_{3}$ ) $\delta$ 210.0, 175.5, 150.7, 145.6, 136.0, 134.2, 131.5, 129.6, $129.2,126.0,121.7,68.9,52.9,49.8,40.4,35.0,34.8,34.6,31.3,30.0,26.1,20.1$.

FTIR ( $\mathrm{NaCl}$, thin film) 3356, 3039, 2953, 2933, 2866, 1705, 1687, 1616, 1596, 1574, 1525, 1496, 1472 , 1426, 1391, 1341, 1312, 1279, 1250, 1223, 1134, 1124, 1038, 1027, 905, 831, 795, 753, 664, $643 \mathrm{~cm}^{-1}$

HRMS (MM) calc'd for $\mathrm{C}_{22} \mathrm{H}_{25} \mathrm{~N}_{2} \mathrm{O}_{2}[\mathrm{M}+\mathrm{H}]^{+} 349.1911$, found 349.1916. 
Preparation of dioxolanes S2 \& S3.

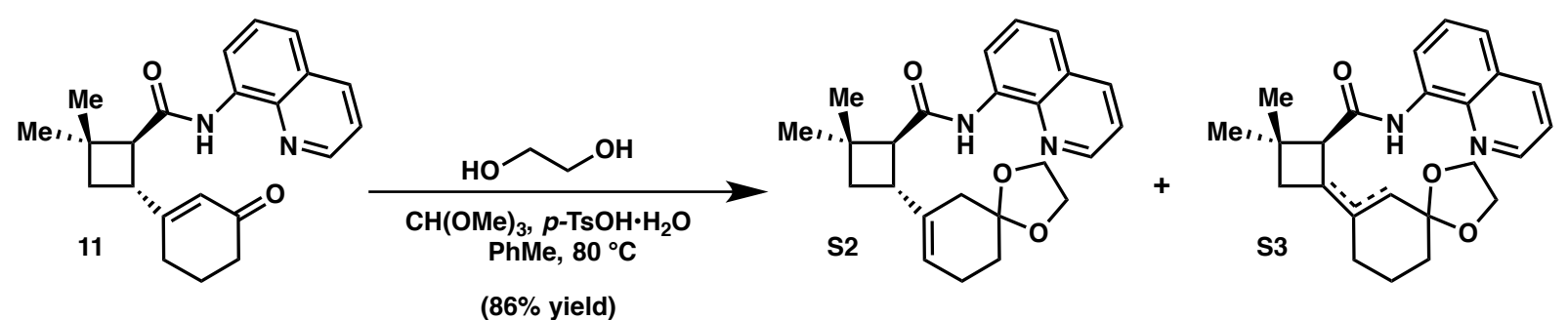

To a flame-dried $200 \mathrm{~mL}$ round-bottom flask was added trans-cyclobutane 11 (2.59 g, 7.43 $\mathrm{mmol}$ ) and the atmosphere was exchanged for $\mathrm{N}_{2}$ three times. Dry PhMe $(74 \mathrm{~mL})$ was then added, followed by ethylene glycol $(16.6 \mathrm{~mL}, 297 \mathrm{mmol}, 40.0$ equiv) and trimethyl orthoformate $(2.44 \mathrm{~mL}, 22.3$ mmol, 3.00 equiv) via syringe. Finally, $p$-toluenesulfonic acid monohydrate $(141 \mathrm{mg}, 0.743 \mathrm{mmol}, 0.10$ equiv) was added as a solid in one portion under a stream of $\mathrm{N}_{2}$. The reaction mixture was heated to $80{ }^{\circ} \mathrm{C}$ for 15 hours, at which point the reaction mixture was cooled to room temperature and quenched with a saturated solution of aqueous $\mathrm{NaHCO}_{3}$. The layers were separated and the aqueous layer was extracted with EtOAc three times. The combined organic extracts were dried over anhydrous $\mathrm{MgSO}_{4}$, filtered, and concentrated in vacuo. The crude residue was purified by silica gel flash chromatography (isocratic: $20 \%$ EtOAc/hexane $\left.+1 \% \mathrm{Et}_{3} \mathrm{~N}\right)$ to afford dioxolanes $\mathbf{S 2}$ and $\mathbf{S 3}(2.50 \mathrm{~g}, 86 \%$ yield) as a partially separable mixture of inconsequential olefin isomers. An analytically pure sample of the major dioxlane (S2) was obtained and a representative spectrum of the mixture as used in the next step is also provided.

Data for $\mathbf{S 2}$ (major product, peak 1): $[\alpha]_{D}^{25.0}=-80.5^{\circ}\left(\mathrm{c}=1.40, \mathrm{CHCl}_{3}\right)$

${ }^{1}$ H NMR $\left(400 \mathrm{MHz}, \mathrm{CDCl}_{3}\right) \delta 9.71(\mathrm{~s}, 1 \mathrm{H}), 8.78(\mathrm{dd}, J=11.0,1.6 \mathrm{~Hz}, 1 \mathrm{H}), 8.78(\mathrm{~d}, J=1.6 \mathrm{~Hz}, 1 \mathrm{H})$, $8.14(\mathrm{dd}, J=8.3,1.7 \mathrm{~Hz}, 1 \mathrm{H}), 7.50(\mathrm{dt}, J=15.8,8.2,7.5 \mathrm{~Hz}, 2 \mathrm{H}), 7.47$ (dd, $J=8.3,1.6 \mathrm{~Hz}, 1 \mathrm{H}), 7.43$ $(\mathrm{dd}, J=8.3,4.2 \mathrm{~Hz}, 1 \mathrm{H}), 5.54(\mathrm{dt}, J=3.6,1.8 \mathrm{~Hz}, 1 \mathrm{H}), 3.96$ (q, $J=4.4,3.9 \mathrm{~Hz}, 4 \mathrm{H}), 3.30$ (q, $J=9.4 \mathrm{~Hz}$, $1 \mathrm{H}), 2.88(\mathrm{~d}, J=9.8 \mathrm{~Hz}, 1 \mathrm{H}), 2.25(\mathrm{~d}, J=2.8 \mathrm{~Hz}, 4 \mathrm{H}), 1.87(\mathrm{dd}, J=10.5,8.6 \mathrm{~Hz}, 1 \mathrm{H}), 1.81-1.61(\mathrm{~m}$, $3 \mathrm{H}), 1.36(\mathrm{~s}, 3 \mathrm{H}), 1.17(\mathrm{~s}, 3 \mathrm{H})$.

${ }^{13}$ C NMR $\left(101 \mathrm{MHz} \mathrm{CDCl}_{3}\right) \delta 170.7,148.2,138.4,137.3,136.3,134.6,127.9,127.4,121.6,121.2$, $119.1,116.3,108.4,64.4,55.2,36.7,36.7,36.4,36.1,30.9,30.8,24.1,23.4$.

FTIR (NaCl, thin film) 3350, 3046, 2952, 2929, 2893, 2839, 1686, 1596, 1578, 1525, 1485, 1460, 1424, $1383,1368,1326,1248,1209,1161,1102,1059,1021,948,826,792,756 \mathrm{~cm}^{-1}$

HRMS (MM) calc'd for $\mathrm{C}_{24} \mathrm{H}_{29} \mathrm{~N}_{2} \mathrm{O}_{3}[\mathrm{M}+\mathrm{H}]^{+}$393.2173, found 393.2188. 


\section{Preparation of vinyl enone (-)-12.}

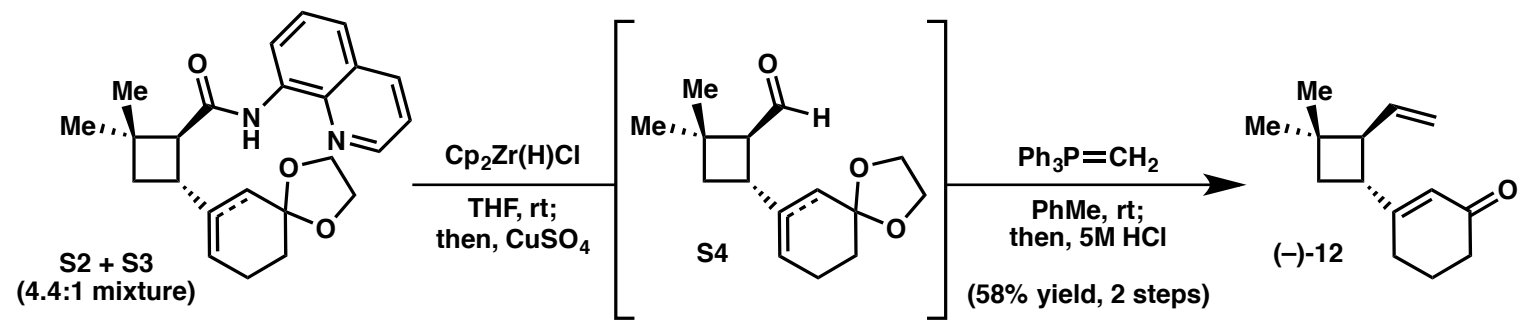

Inside a $\mathrm{N}_{2}$-filled glovebox, two flame-dried $200 \mathrm{~mL}$ round-bottom flasks were each charged with Schwartz's reagent (1.60 g, $6.21 \mathrm{mmol}, 2.04$ equiv) and sealed under $\mathrm{N}_{2}$. The flasks were removed from the glovebox and THF $(15.5 \mathrm{~mL})$ was added to each via syringe. To each of the milky-white suspensions was added a mixture of dioxolanes $\mathbf{S 2}$ and $\mathbf{S 3}(1.19 \mathrm{~g}, 3.04 \mathrm{mmol})$ as a solution in THF (16.0 $\mathrm{mL})$ in a quick drip at room temperature. The mixtures immediately began to turn yellow, darkening to orange over the course of 1 hour, at which point the reactions were quenched with saturated aqueous $\mathrm{NaHCO}_{3}$ and combined together. The layers were separated and the aqueous layer was extracted twice with EtOAc. The combined organic layers were washed twice with $100 \mathrm{~mL}$ of a $0.6 \mathrm{M}$ aqueous solution of $\mathrm{CuSO}_{4}$ to remove the liberated 8-aminoquinoline. The organic layer was then dried over $\mathrm{MgSO}_{4}$, filtered, and concentrated in vacuo. The crude aldehyde (S4, $1.70 \mathrm{~g})$ was dissolved in dry PhMe $(20 \mathrm{~mL})$ and treated with freshly prepared ylide ( $80 \mathrm{~mL}, 32.0 \mathrm{mmol}, 5.26$ equiv) at room temperature. The reaction was stirred for 2 hours and monitored by TLC. Upon complete conversion, the reaction was cooled to $0{ }^{\circ} \mathrm{C}$ and quenched with $5 \mathrm{M} \mathrm{HCl}$. The layers were separated and the aqueous layer was extracted twice with $\mathrm{Et}_{2} \mathrm{O}$. The combined organic layers were concentrated in vacuo and the solvent replaced with THF (30 mL). The dioxolane was hydrolyzed by stirring vigorously with $5 \mathrm{M} \mathrm{HCl}$ for 8 hours, at which time $\mathrm{Et}_{2} \mathrm{O}$ was added and the layers separated. The aqueous layer was extracted twice with $\mathrm{Et}_{2} \mathrm{O}$ and the combined organics washed with aqueous $\mathrm{NaHCO}_{3}$, dried over $\mathrm{MgSO}_{4}$ and concentrated in vacuo. The crude residue was purified by silica gel flash chromatography (isocratic: $30 \% \mathrm{Et}_{2} \mathrm{O} /$ hexanes) to afford vinyl enone (-)12 (715 mg, 58\% yield over 2 steps) as a clear oil: $[\alpha]_{D}^{25.0}=-100^{\circ}\left(\mathrm{c}=1.02, \mathrm{CHCl}_{3}\right)$.

${ }^{1}$ H NMR (400 MHz, $\left.\mathrm{CDCl}_{3}\right) \delta 5.84(\mathrm{q}, J=1.5 \mathrm{~Hz}, 1 \mathrm{H}), 5.81$ (dddt, $\left.J=16.8,10.6,7.9,0.5,0.5 \mathrm{~Hz}, 1 \mathrm{H}\right)$, $5.04(\mathrm{qd}, J=1.9,1.0 \mathrm{~Hz}, 1 \mathrm{H}), 5.01(\mathrm{ddd}, J=10.4,2.0,1.1 \mathrm{~Hz}, 1 \mathrm{H}), 2.88(\mathrm{q}, J=9.7,9.1,9.0 \mathrm{~Hz}, 1 \mathrm{H})$, 2.48 (ddq, $J=9.8,7.9,1.0 \mathrm{~Hz}, 1 \mathrm{H}), 2.34$ (t, $J=7.0,6.5 \mathrm{~Hz}, 2 \mathrm{H}), 2.21$ (qdd, $J=5.9,1.5,0.8 \mathrm{~Hz}, 2 \mathrm{H}$ ), 1.95 (dt, $J=7.7,6.1 \mathrm{~Hz}, 2 \mathrm{H}), 1.85(\mathrm{ddd}, J=10.7,8.3,0.8 \mathrm{~Hz}, 1 \mathrm{H}), 1.67$ (t, $J=10.4 \mathrm{~Hz}, 1 \mathrm{H}), 1.05$ (s, 3H), 1.04 $(\mathrm{s}, 3 \mathrm{H})$.

${ }^{13}$ C NMR $\left(101 \mathrm{MHz}, \mathrm{CDCl}_{3}\right) \delta 200.1,168.7,137.4,123.4,116.1,53.8,41.1,37.5,37.3,36.0,30.0,27.9$, 23.1, 22.6. 
FTIR ( $\mathrm{NaCl}$, thin film) 3320, 3076, 3039, 2953, 2934, 2891, 2866, 2827, 1671, 1622, 1456, 1428, 1417 , $1382,1368,1346,1324,1290,1251,1191,1124,995,968,942,912,886,755 \mathrm{~cm}^{-1}$

HRMS (MM) calc'd for $\mathrm{C}_{14} \mathrm{H}_{21} \mathrm{O}[\mathrm{M}+\mathrm{H}]^{+}$205.1587, found 205.1587.

\section{Preparation of Wittig ylide.}

Inside a $\mathrm{N}_{2}$-filled glovebox, methyltriphenylphosphonium bromide $(22.2 \mathrm{~g}, 62.1 \mathrm{mmol})$ and $\mathrm{KO} t$ $\mathrm{Bu}$ (7.36 g, $65.6 \mathrm{mmol}, 1.06$ equiv) were added to a flame-dried $500 \mathrm{~mL}$ round-bottom flask and sealed under nitrogen. The flask was brought out of the box and dry PhMe $(155 \mathrm{~mL})$ was added via syringe. The flask was fitted with a reflux condenser under a stream of $\mathrm{N}_{2}$ and heated to $110^{\circ} \mathrm{C}$ for 4 hours, at which time the reaction was cooled to room temperature and the salts were allowed to settle for 3 hours before the bright yellow supernatant ( $\sim 0.40 \mathrm{M}$ salt-free ylide) was used for the methylenation of crude transaldehyde S4, as well as purified cis/trans-aldehyde $\mathbf{S 5}$.

\section{Preparation of protected iodide 13.}

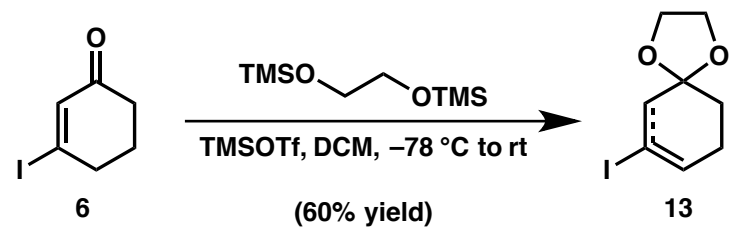

Inside a $\mathrm{N}_{2}$-filled glove box, a $250 \mathrm{~mL}$ round bottom flask was charged with TMSOTf $(0.410 \mathrm{~mL}$, $0.230 \mathrm{mmol}, 0.010$ equiv) and DCM $(20.0 \mathrm{~mL})$. The flask was sealed, removed from the glove box, and placed under a $\mathrm{N}_{2}$ atmosphere. The reaction mixture was cooled to $-78{ }^{\circ} \mathrm{C}$, and 1,2bistrimethylsilyloxyethane $(11.0 \mathrm{~mL}, 45.0 \mathrm{mmol}, 2.00$ equiv) was added via syringe. (Note, best results were obtained when 1,2-bistrimethylsilyloxyethane was sparged with argon for 30 min prior to addition). Vinyl iodide 6 (5.00 g, $22.5 \mathrm{mmol}, 1.00$ equiv) was added to the flask dropwise as a solution in DCM $(20.0 \mathrm{~mL})$, via cannula transfer. An additional portion of DCM $(5.00 \mathrm{~mL})$ was used to complete the transfer. The colorless reaction mixture was allowed to stir at $-78{ }^{\circ} \mathrm{C}$ for 1 hour, at which point the reaction mixture was warmed to $0{ }^{\circ} \mathrm{C}$. The reaction mixture became yellow immediately upon warming and was allowed to warm to room temperature over 16 hours. The reaction mixture became dark orange and was quenched with the addition of DIPEA $(11.0 \mathrm{~mL})$, at which point the reaction became yellow. The mixture was poured into a separatory funnel and diluted with saturated $\mathrm{NaHCO}_{3}$ solution. The aqueous layer was extracted three times with DCM. The organic layers were combined, dried over a 1:1 mixture of anhydrous $\mathrm{K}_{2} \mathrm{CO}_{3}$ and $\mathrm{Na}_{2} \mathrm{SO}_{4}$, filtered, and concentrated to provide a yellow residue that was purified by flash silica gel chromatography (5\% EtOAc, $1 \% \mathrm{Et}_{3} \mathrm{~N} / \mathrm{h}$ exane - 20\% EtOAc, $1 \% \mathrm{Et}_{3} \mathrm{~N} /$ hexane) to provide 13 (3.61 g, 60\% yield) as a 8:1 mixture of olefin isomers, as a pale yellow oil. 
${ }^{1} \mathbf{H}$ NMR $\left(400 \mathrm{MHz}, \mathrm{CDCl}_{3}\right) \delta 6.34(\mathrm{tt}, J=4.0,1.9 \mathrm{~Hz}, 1 \mathrm{H}), 3.98(\mathrm{p}, J=1.7 \mathrm{~Hz}, 4 \mathrm{H}), 2.72(\mathrm{q}, J=2.3 \mathrm{~Hz}$, $2 \mathrm{H}), 2.36-2.22(\mathrm{~m}, 2 \mathrm{H}), 1.77(\mathrm{t}, J=6.5 \mathrm{~Hz}, 2 \mathrm{H})$.

${ }^{13}$ C NMR $\left(101 \mathrm{MHz}, \mathrm{CDCl}_{3}\right) \delta 136.6,108.1,91.1,64.7,49.3,30.2,27.5$.

FTIR ( $\mathrm{NaCl}$, thin film) 3040, 2955, 2934, 2881, 2836, 2684, 1637, 1474, 1443, 1429, 1418, 1360, 1330, $1300,1243,1207,1142,1114,1076,1058,1021,970,948,889,848,827,776,738,662 \mathrm{~cm}^{-1}$

HRMS (FAB) calc'd for $\mathrm{C}_{8} \mathrm{H}_{11} \mathrm{IO}_{2}[\mathrm{M}]^{+} 266.9876$, found 266.9888 .

\section{Preparation of cis-dioxolane 14.}

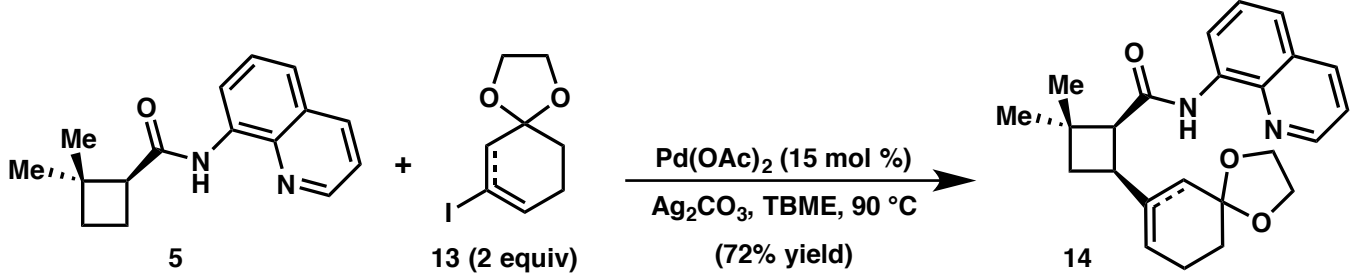

A $100 \mathrm{~mL}$, thick-walled pressure vessel was charged with $\mathrm{Pd}(\mathrm{OAc})_{2}(132 \mathrm{mg}, 0.590 \mathrm{mmol}, 0.150$ equiv), $\mathrm{Ag}_{2} \mathrm{CO}_{3}$ (1.08 g, $3.93 \mathrm{mmol}, 1.00$ equiv), and 5 (1.00 g, $3.93 \mathrm{mmol}, 1.00$ equiv). Vinyl iodide 13 (2.09 g, $7.86 \mathrm{mmol}, 2.00$ equiv) was then added to the flask as a solution in TBME $(19.7 \mathrm{~mL})$. The reaction vessel was sealed with a screw top under ambient conditions and heated to $90{ }^{\circ} \mathrm{C}$ in an oil bath. The heterogeneous reaction mixture is olive green upon addition of vinyl iodide. After heating for five minutes, the reaction mixture became black. After 16 hours, the flask was removed from the oil bath and allowed to cool to room temperature. The reaction mixture was filtered over a pad of celite and the filter cake was washed with DCM. The filtrate was concentrated, and the crude orange residue was purified by flash silica gel chromatography (30\% EtOAc, $1 \% \mathrm{Et}_{3} \mathrm{~N} / \mathrm{h}$ exane-35\% EtOAc, $+1 \% \mathrm{Et}_{3} \mathrm{~N} / \mathrm{hexane}$ ) to provide $14(1.11 \mathrm{~g}, 72 \%$ yield $)$ as a white foam: $[\alpha]_{D}^{25.0}=-29.3^{\circ}\left(\mathrm{c}=1.95, \mathrm{CHCl}_{3}\right)$.

${ }^{1}$ H NMR $\left(400 \mathrm{MHz}, \mathrm{CDCl}_{3}\right) \delta 9.87(\mathrm{~s}, 1 \mathrm{H}), 8.81(\mathrm{ddd}, J=24.2,7.3,1.5 \mathrm{~Hz}, 2 \mathrm{H}), 8.13(\mathrm{dd}, J=8.3,1.7$ $\mathrm{Hz}, 1 \mathrm{H}), 7.49(\mathrm{td}, J=8.2,7.5,6.6 \mathrm{~Hz}, 1 \mathrm{H}), 7.45(\mathrm{dd}, J=8.3,1.6 \mathrm{~Hz}, 1 \mathrm{H}), 7.42(\mathrm{dd}, J=8.3,4.2 \mathrm{~Hz}, 1 \mathrm{H})$, $5.65(\mathrm{dd}, J=17.8,9.1 \mathrm{~Hz}, 1 \mathrm{H}), 4.03-3.81(\mathrm{~m}, 2 \mathrm{H}), 3.76-3.64(\mathrm{~m}, 2 \mathrm{H}), 3.25(\mathrm{q}, J=9.1,8.2 \mathrm{~Hz}, 1 \mathrm{H})$, $3.05(\mathrm{dd}, J=8.7,2.8 \mathrm{~Hz}, 1 \mathrm{H}), 2.46(\mathrm{t}, J=10.9 \mathrm{~Hz}, 1 \mathrm{H}), 2.40-2.23(\mathrm{~m}, 2 \mathrm{H}), 2.19$ (dt, $J=16.5,2.1 \mathrm{~Hz}$, $1 \mathrm{H}), 2.06-1.97(\mathrm{~m}, 1 \mathrm{H}), 1.93(\mathrm{ddd}, J=11.1,8.3,2.9 \mathrm{~Hz}, 1 \mathrm{H}), 1.71-1.59(\mathrm{~m}, 1 \mathrm{H}), 1.57-1.48(\mathrm{~m}, 1 \mathrm{H})$, $1.40(\mathrm{~s}, 3 \mathrm{H}), 1.11(\mathrm{~s}, 3 \mathrm{H})$.

${ }^{13}$ C NMR $\left(101 \mathrm{MHz} \mathrm{CDCl}_{3}\right) \delta 171.0,147.9,138.7,136.3,134.9,134.8,127.9,127.5,121.4,121.2$, $120.8,116.6,108.3,64.3,64.1,55.9,37.2,37.1,36.7,35.4,30.8,30.2,25.1,24.4$. 
FTIR (NaCl, thin film) 3357, 3300, 3043, 3006, 2952, 2928, 2881, 1685, 1664, 1596, 1577, 1523, 1485, $1460,1424,1385,1324,1255,1208,1160,1132,1106,1060,1039,1020,947,846,826,792,755,666$ $\mathrm{cm}^{-1}$

HRMS (MM) calc'd for $\mathrm{C}_{24} \mathrm{H}_{29} \mathrm{~N}_{2} \mathrm{O}_{3}[\mathrm{M}+\mathrm{H}]^{+} 393.2173$, found 393.2183.

\section{Preparation of trans-aldehyde (15).}

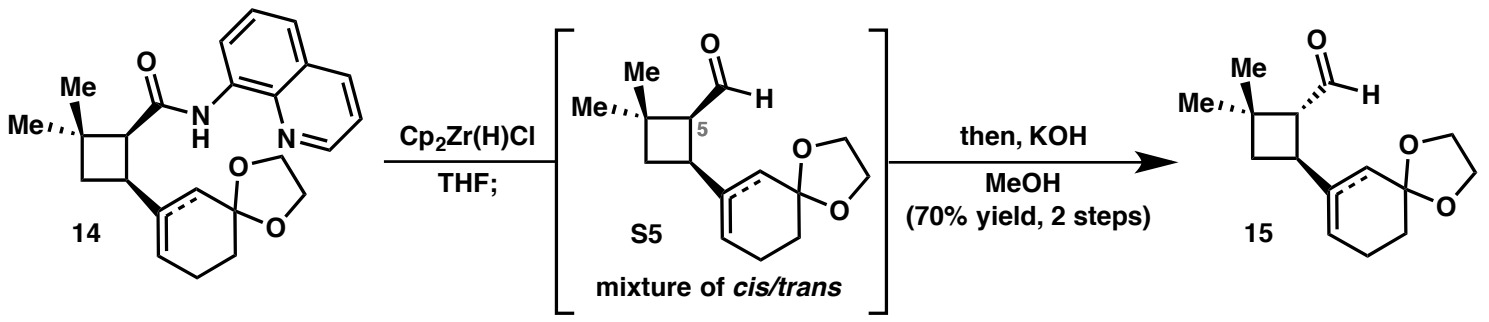

Inside a $\mathrm{N}_{2}$-filled glove box, a $250 \mathrm{~mL}$ round bottom flask was charged with Schwartz's reagent (2.30 g, $8.92 \mathrm{mmol}, 2.06$ equiv) and THF (22.3 mL). Cis-dioxolane 14 (1.70 g, $4.32 \mathrm{mmol}, 1.00$ equiv) was added to the flask as a solution in THF $(22.3 \mathrm{~mL})$. The flask was sealed, removed from the glove box and put under a $\mathrm{N}_{2}$ atmosphere. The flask was covered with aluminum foil and allowed to stir for one hour, at which point the reaction was quenched with the addition of saturated $\mathrm{NaHCO}_{3}$ solution. (Note, it is important that the quench be conducted very quickly to avoid decomposition of excess Schwartz's reagent and formation of $\mathrm{HCl}$ ). The reaction mixture was diluted with EtOAc and the organic layer separated. The aqueous layer was filtered through a pad of celite and sand and then extracted 5x with EtOAc. The combined organics were dried over anhydrous $\mathrm{Na}_{2} \mathrm{SO}_{4}$, filtered, and concentrated to provide a yellow residue that was purified by flash silica gel chromatography (15\% EtOAc, $\left.1 \% \mathrm{Et}_{3} \mathrm{~N} / \mathrm{hexanes}\right)$ to provide S5 (755 $\mathrm{mg}, 3.01 \mathrm{mmol})$ as a yellow oil as a 1.8:1 (cis/trans) mixture of diastereomers at C5. The oil was concentrated directly into a $200 \mathrm{~mL}$ round bottom flask and dissolved in wet $\mathrm{MeOH}(60.0 \mathrm{~mL})$. The flask was then charged with $\mathrm{KOH}(3.36 \mathrm{~g}, 59.9 \mathrm{mmol}, 20.0$ equiv) and the mixture allowed to stir for 1 hour at room temperature. The mixture was then concentrated to a volume of $\sim 3 \mathrm{~mL}$ and diluted with pH 7 buffer. A pale yellow precipitate formed upon addition of buffer. The solution was slowly acidified using dilute citric acid until $\mathrm{pH} 7$ was achieved. The mixture was then poured into a separatory funnel and extracted $3 \mathrm{x}$ with EtOAc. The combined organics were dried over anhydrous $\mathrm{Na}_{2} \mathrm{SO}_{4}$, filtered, and concentrated to provide trans-aldehyde 15 (755 mg, 70\% over 2 steps) as a mixture of olefin isomers. The yellow oil was analytically pure and used directly in the next step: $[\alpha]_{D}^{25.0}=+35.2^{\circ}\left(\mathrm{c}=0.295, \mathrm{CHCl}_{3}\right)$. Note: it is recommended that the aldehyde be used immediately in the next step to avoid decomposition.

${ }^{1}$ H NMR $\left(400 \mathrm{MHz}, \mathrm{CDCl}_{3}\right) \delta 9.76(\mathrm{t}, J=2.2 \mathrm{~Hz}, 1 \mathrm{H}), 5.37(\mathrm{dt}, J=3.8,1.9 \mathrm{~Hz}, 1 \mathrm{H}), 3.97(\mathrm{dd}, J=2.5$, $1.3 \mathrm{~Hz}, 4 \mathrm{H}), 3.14(\mathrm{q}, J=9.2 \mathrm{~Hz}, 1 \mathrm{H}), 2.73(\mathrm{dt}, J=10.0,2.1 \mathrm{~Hz}, 1 \mathrm{H}), 2.22(\mathrm{dp}, J=6.5,2.1 \mathrm{~Hz}, 2 \mathrm{H}), 2.16$ 
- $1.97(\mathrm{~m}, 2 \mathrm{H}), 1.77$ (ddd, $J=15.5,9.4,2.0 \mathrm{~Hz}, 2 \mathrm{H}), 1.69$ (td, $J=6.5,2.1 \mathrm{~Hz}, 2 \mathrm{H}), 1.24(\mathrm{~s}, 3 \mathrm{H}), 1.14(\mathrm{~s}$, $3 \mathrm{H})$.

${ }^{13}$ C NMR $\left(101 \mathrm{MHz}, \mathrm{CDCl}_{3}\right) \delta 203.2,137.1,118.8,108.3,64.4,59.5,37.3,37.1,36.7,34.3,31.2,30.7$, 24.0, 24.0.

FTIR $(\mathrm{NaCl}$, thin film) 2954, 2929, 2896, 2873, 2707, 1712, 1670, 1577, 1522, 1461,1449, 1434, 1420, $1383,1367,1340,1312,1297,1249,1209,1179,1103,1059,1039,1018,948,846,793 \mathrm{~cm}^{-1}$

HRMS (MM) calc'd for $\mathrm{C}_{15} \mathrm{H}_{23} \mathrm{O}_{3}[\mathrm{M}+\mathrm{H}]^{+} 251.1642$, found 251.1645 .

\section{Preparation of vinyl enone $(+)-12$}

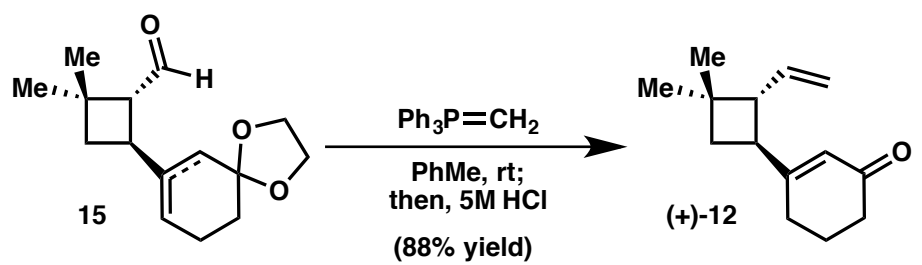

A $250 \mathrm{~mL}$ round bottom flask was charged with aldehyde, 15 (720 mg, $2.88 \mathrm{mmol}, 1.00$ equiv). The flask was evacuated and backfilled three times with $\mathrm{N}_{2}$ and charged with toluene $(2.30 \mathrm{~mL})$. The flask was then charged with freshly prepared ylide solution ${ }^{7}(36.0 \mathrm{~mL}, 0.4 \mathrm{M}, 5.00$ equiv) and the reaction mixture was allowed to stir for 30 minutes at room temperature. The reaction was quenched with the addition of saturated $\mathrm{NaHCO}_{3}$ solution $(10.0 \mathrm{~mL})$. The organic layer was separated and the aqueous layer extracted $3 \mathrm{x}$ with $\mathrm{Et}_{2} \mathrm{O}$. The combined organics were concentrated and dissolved in a 1:1 mixture of THF and $5 \mathrm{M} \mathrm{HCl}(28 \mathrm{~mL} .0)$. The reaction mixture was allowed to stir over 16 hours, at which point the mixture was diluted with $\mathrm{Et}_{2} \mathrm{O}$ and water. The layers were separated and the aqueous layer extracted $3 \mathrm{x}$ with $\mathrm{Et}_{2} \mathrm{O}$. The combined organics were dried over anhydrous $\mathrm{MgSO}_{4}$, filtered, and concentrated. The crude yellow residue was purified by flash silica gel chromatography (20-30\% $\mathrm{Et}_{2} \mathrm{O} /$ pentane) to provide $(+)-12(520 \mathrm{mg}, 88 \%)$ as a pale yellow oil: $[\alpha]_{D}^{25.0}=+102^{\circ}\left(\mathrm{c}=0.705, \mathrm{CHCl}_{3}\right)$.

${ }^{1} \mathbf{H}$ NMR (400 MHz, $\left.\mathrm{CDCl}_{3}\right) \delta 5.84(\mathrm{q}, J=1.6 \mathrm{~Hz}, 1 \mathrm{H}), 5.81$ (dddt, $\left.J=16.8,10.6,7.9,0.5,0.5 \mathrm{~Hz}, 1 \mathrm{H}\right)$, $5.04(\mathrm{qd}, J=1.9,1.0 \mathrm{~Hz}, 1 \mathrm{H}), 5.01(\mathrm{ddd}, J=10.8,1.9,1.1 \mathrm{~Hz}, 1 \mathrm{H}), 2.88(\mathrm{q}, J=9.7,9.1,9.0 \mathrm{~Hz}, 1 \mathrm{H})$, 2.48 (ddq, $J=9.8,7.9,1.0 \mathrm{~Hz}, 1 \mathrm{H}), 2.34$ (t, $J=7.0,6.5 \mathrm{~Hz}, 2 \mathrm{H}), 2.20$ (qdd, $J=6.0,1.5,0.8 \mathrm{~Hz}, 2 \mathrm{H}), 1.95$ (dt, $J=7.7,6.1 \mathrm{~Hz}, 2 \mathrm{H}), 1.85(\mathrm{ddd}, J=10.8,8.3,0.8 \mathrm{~Hz}, 1 \mathrm{H}), 1.67$ (t, $J=10.3 \mathrm{~Hz}, 1 \mathrm{H}), 1.05$ (s, 3H), 1.04 (s, 3H). 
${ }^{13}$ C NMR $\left(101 \mathrm{MHz}, \mathrm{CDCl}_{3}\right) \delta 200.1,168.8,137.4,123.4,116.1,53.8,41.1,37.5,37.3,36.0,30.0,27.9$, 23.1, 22.6.

FTIR ( $\mathrm{NaCl}$, thin film) 3320, 3076, 3039, 2953, 2934, 2891, 2866, 2827, 1671, 1622, 1456, 1428, 1417 , $1382,1368,1346,1324,1290,1251,1191,1124,995,968,942,912,886,755 \mathrm{~cm}^{-1}$

HRMS (MM) calc'd for $\mathrm{C}_{14} \mathrm{H}_{21} \mathrm{O}[\mathrm{M}+\mathrm{H}]^{+}$205.1587, found 205.1587.

\section{Preparation of vinyl ketone 4.}

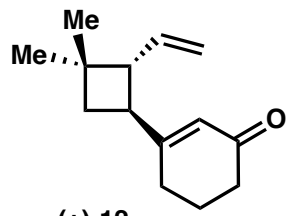

$(+)-12$

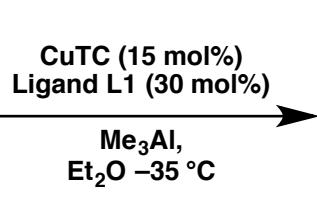

$(94 \%, 19: 1 \mathrm{dr})$

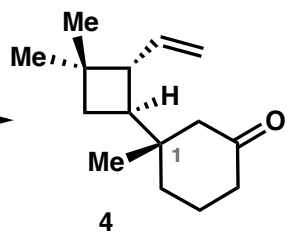

4

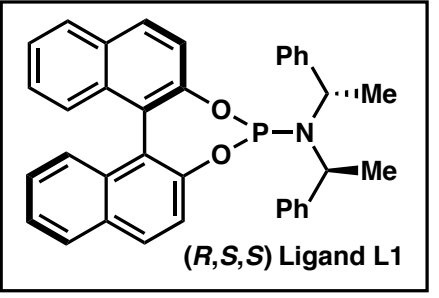

$(R, S, S)$ Ligand L1

Inside a $\mathrm{N}_{2}$-filled glovebox, CuTC (105 mg, $0.551 \mathrm{mmol}, 0.150$ equiv) and ( $\left.R, S, S\right)$-ligand $\mathbf{L 1}^{8}$ (594 mg, $1.10 \mathrm{mmol}, 0.30$ equiv) were added to a flame dried $100 \mathrm{~mL}$ round-bottom flask. The reagents were suspended in $\mathrm{Et}_{2} \mathrm{O}(18.0 \mathrm{~mL})$ and stirred at room temperature for 30 minutes before vinyl enone $(+)$ $12(750 \mathrm{mg}, 3.67 \mathrm{mmol})$ was added as a solution in $\mathrm{Et}_{2} \mathrm{O}(18.0 \mathrm{~mL})$. The reaction was sealed under $\mathrm{N}_{2}$, removed from the glovebox and placed under a balloon atmosphere of argon. The reaction mixture was allowed to equilibrate to $-35{ }^{\circ} \mathrm{C}$ for 5 minutes using a cryocool unit to maintain the temperature. $\mathrm{Me}_{3} \mathrm{Al}$ (2.0 $\mathrm{M}$ in heptane; $3.67 \mathrm{~mL}, 7.34 \mathrm{mmol}, 2.00$ equiv) was then added dropwise and the reaction stirred at $-35{ }^{\circ} \mathrm{C}$ for 17 hours, at which point wet $\mathrm{MeOH}(5 \mathrm{~mL})$ was slowly added to quench excess $\mathrm{Me}_{3} \mathrm{Al}$. The mixture was warmed to room temperature, filtered over a plug of silica gel and washed thoroughly with $\mathrm{Et}_{2} \mathrm{O}$ and DCM (until no product remained in eluent). The filtrate was concentrated in vacuo and the crude residue purified by silica gel flash chromatography (isocratic: 20\% hexane/DCM) to afford vinyl ketone 4 (760 mg, 94\% yield) as a 19:1 mixture of inseparable diastereomers at C1, colorless oil. Note: this 19:1 mixture is carried through the next three reactions, and a single diastereomer at $\mathrm{C} 1$ is isolable after the ring-closing metathesis: $[\alpha]_{D}^{25.0}=+37.6^{\circ}\left(\mathrm{c}=1.05, \mathrm{CHCl}_{3}\right)$.

${ }^{1}$ H NMR (400 MHz, $\left.\mathrm{C}_{6} \mathrm{D}_{6}\right) \delta 5.68(\mathrm{ddd}, J=16.9,10.5,8.6 \mathrm{~Hz}, 1 \mathrm{H}), 4.96(\mathrm{qd}, J=2.2,0.8 \mathrm{~Hz}, 1 \mathrm{H}), 4.93$ (ddd, $J=11.3,2.2,0.8 \mathrm{~Hz}, 1 \mathrm{H}), 2.20$ (ddt, $J=9.6,8.6,0.9 \mathrm{~Hz}, 1 \mathrm{H}), 2.11$ (dtt, $J=13.9,4.8,1.4 \mathrm{~Hz}, 1 \mathrm{H}$ ), $1.92(\mathrm{dd}, J=3.3,1.7 \mathrm{~Hz}, 2 \mathrm{H}), 1.90-1.79(\mathrm{~m}, 2 \mathrm{H}), 1.50-1.40(\mathrm{~m}, 2 \mathrm{H}), 1.31-1.21(\mathrm{~m}, 2 \mathrm{H}), 1.21-1.13$ (m, 1H), 0.99 (dtt, $J=13.4,4.7,4.5,1.5,1.1 \mathrm{~Hz}, 1 \mathrm{H}), 0.94(\mathrm{~s}, 3 \mathrm{H}), 0.93(\mathrm{~s}, 3 \mathrm{H}), 0.66(\mathrm{~s}, 3 \mathrm{H})$.

${ }^{1}$ H NMR $\left(400 \mathrm{MHz}, \mathrm{CDCl}_{3}\right) \delta 5.74(\mathrm{ddd}, J=17.1,10.3,8.7 \mathrm{~Hz}, 1 \mathrm{H}), 5.08-4.81(\mathrm{~m}, 2 \mathrm{H}), 2.33(\mathrm{ddd}, J=$ 9.6, 8.7, $0.9 \mathrm{~Hz}, 1 \mathrm{H}), 2.30-2.19(\mathrm{~m}, 2 \mathrm{H}), 2.16(\mathrm{~d}, J=13.5 \mathrm{~Hz}, 1 \mathrm{H}), 2.07$ (td, $J=10.1,8.5 \mathrm{~Hz}, 1 \mathrm{H}), 1.99$ 
(dt, $J=13.4,1.8 \mathrm{~Hz}, 1 \mathrm{H}), 1.90(\mathrm{ddq}, J=14.0,6.2,4.7 \mathrm{~Hz}, 1 \mathrm{H}), 1.85-1.72(\mathrm{~m}, 1 \mathrm{H}), 1.56$ (ddd, $J=13.6$, 11.1, $4.4 \mathrm{~Hz}, 1 \mathrm{H}), 1.51-1.34(\mathrm{~m}, 3 \mathrm{H}), 0.98$ (s, 3H), 0.97 (s, 3H), 0.83 (s, 3H).

${ }^{13}$ C NMR (101 MHz, $\left.\mathrm{CDCl}_{3}\right) \delta 212.8,139.6,115.3,51.2,49.5,45.2,41.3,40.1,34.8,34.0,33.0,30.1$, 23.7, 22.1, 21.8.

${ }^{13}$ C NMR (101 MHz, $\left.\mathrm{C}_{6} \mathrm{D}_{6}\right) \delta 209.3,139.9,115.3,51.0,49.5,45.2,41.2,39.7,34.7,33.9,32.9,30.1$, 23.7, 22.1, 21.8.

FTIR ( $\mathrm{NaCl}$, thin film) 3075, 2953, 2873, 1713, 1633, 1460, 1422, 1382, 1368, 1312, 1285, 1253, 1228, $1172,1049,995,910 \mathrm{~cm}^{-1}$

HRMS (FAB) calc'd for $\mathrm{C}_{15} \mathrm{H}_{24} \mathrm{O}[\mathrm{M}]^{+} 221.1900$, found 221.1897 .

\section{Preparation of exo-enone 17.}

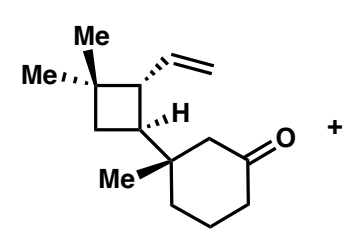

4

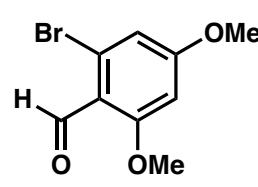

16

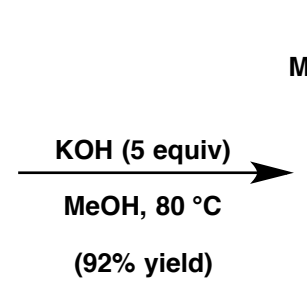

(92\% yield)

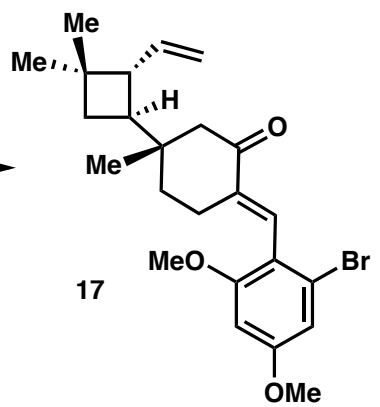

To a $200 \mathrm{~mL}$ round-bottom flask were added vinyl ketone 4 (884 mg, $4.01 \mathrm{mmol}$ ), aryl aldehyde $\mathbf{1 6}^{9}$ (1.08 g, $4.41 \mathrm{mmol}, 1.10$ equiv), and $\mathrm{KOH}$ (1.13 g, $20.1 \mathrm{mmol}, 5.00$ equiv). Freshly distilled $\mathrm{MeOH}$ $(40.1 \mathrm{~mL})$ was then added, the flask fitted with a reflux condenser under ambient conditions and heated to $80{ }^{\circ} \mathrm{C}$ for 12 hours. At completion, the volume of $\mathrm{MeOH}$ was reduced in vacuo and the reaction quenched with a saturated solution of aqueous $\mathrm{NH}_{4} \mathrm{Cl}$. $\mathrm{Et}_{2} \mathrm{O}$ was added and the layers were separated. The aqueous layer was extracted twice with $\mathrm{Et}_{2} \mathrm{O}$ and the combined organic layers were dried over $\mathrm{MgSO}_{4}$, filtered and concentrated in vacuo. The crude residue was purified by silica gel flash chromatography (isocratic: $20 \%$ $\mathrm{Et}_{2} \mathrm{O} /$ hexane) to afford exo-enone $\mathbf{1 7}(1.66 \mathrm{~g}, 92 \%$ yield $)$ as an off-white solid: $[\alpha]_{D}^{25.0}=+11.4^{\circ}(\mathrm{c}=1.08$, $\left.\mathrm{CHCl}_{3}\right)$.

${ }^{1}$ H NMR $\left(400 \mathrm{MHz}, \mathrm{CDCl}_{3}\right) \delta 7.11(\mathrm{t}, J=2.0 \mathrm{~Hz}, 1 \mathrm{H}), 6.75(\mathrm{~d}, J=2.3 \mathrm{~Hz}, 1 \mathrm{H}), 6.40(\mathrm{~d}, J=2.3 \mathrm{~Hz}, 1 \mathrm{H})$, $5.74(\mathrm{ddd}, J=17.1,10.3,8.6 \mathrm{~Hz}, 1 \mathrm{H}), 4.95(\mathrm{ddd}, J=24.7,2.1,0.8 \mathrm{~Hz}, 1 \mathrm{H}), 4.94(\mathrm{td}, J=2.3,0.8 \mathrm{~Hz}, 1 \mathrm{H})$, $3.80(\mathrm{~s}, 3 \mathrm{H}), 3.75(\mathrm{~s}, 3 \mathrm{H}), 2.44-2.20(\mathrm{~m}, 5 \mathrm{H}), 2.12(\mathrm{td}, J=10.0,8.5 \mathrm{~Hz}, 1 \mathrm{H}), 1.55(\mathrm{ddd}, J=13.3,10.3$, $6.0 \mathrm{~Hz}, 1 \mathrm{H}), 1.50(\mathrm{ddd}, J=10.7,8.4,0.6 \mathrm{~Hz}, 1 \mathrm{H}), 1.45(\mathrm{~d}, J=10.4 \mathrm{~Hz}, 1 \mathrm{H}), 1.38(\mathrm{dtd}, J=11.0,5.0,2.1$ $\mathrm{Hz}, 1 \mathrm{H}), 0.99$ (s, 3H), 0.98 (s, 3H), 0.93 (s, 3H). 
${ }^{13} \mathbf{C}$ NMR $\left(101 \mathrm{MHz}, \mathrm{CDCl}_{3}\right) \delta 202.2,160.8,158.6,139.6,139.2,130.5,125.1,118.7,115.3,109.0,98.1$, $55.8,55.7,50.3,49.4,45.0,36.5,34.9,33.2,32.5,30.1,24.7,23.7,22.6$.

FTIR ( $\mathrm{NaCl}$, thin film) 3073, 3000, 2952, 2863, 1686, 1599, 1558, 1482, 1461, 1435, 1407, 1381, 1367, $1303,1259,1214,1153,1051,1035,996,938,911,960,831,795 \mathrm{~cm}^{-1}$

HRMS (MM) calc'd for $\mathrm{C}_{24} \mathrm{H}_{32} \mathrm{BrO}_{3}[\mathrm{M}+\mathrm{H}]^{+}$447.1529, found 447.1520.

\section{Preparation of allylic alcohols 18 and S6.}
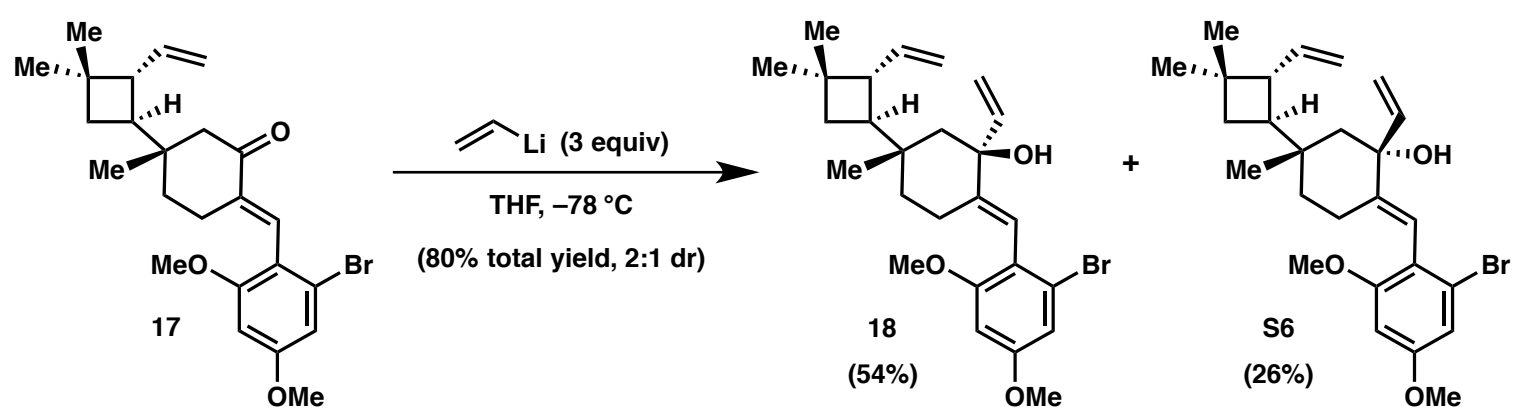

A $100 \mathrm{~mL}$ round-bottom flask was flame dried under vacuum and backfilled with $\mathrm{N}_{2}$. Dry THF $(21.2 \mathrm{~mL})$ was then added, followed by freshly prepared vinyllithium as a solution in THF $(8.42 \mathrm{~mL}$, $0.756 \mathrm{M}, 3.00$ equiv). The solution was cooled to $-78{ }^{\circ} \mathrm{C}$ and exo-enone 17 (928 $\mathrm{mg}, 2.07 \mathrm{mmol}$ ) was taken up in $5.0 \mathrm{~mL}$ THF and added dropwise over 5 minutes. After 40 minutes, the reaction was quenched with a saturated solution of $\mathrm{NH}_{4} \mathrm{Cl}$ and warmed to room temperature. The mixture was diluted with $\mathrm{Et}_{2} \mathrm{O}$ and the layers were separated. The aqueous layer was extracted twice with $\mathrm{Et}_{2} \mathrm{O}$ and the combined organic layers were dried over $\mathrm{MgSO}_{4}$, filtered and concentrated in vacuo. The crude residue was purified by silica gel flash chromatography (30\% hexane/DCM + 1\% EtOAc until unreacted $\mathbf{1 7}$ and S6 elute, then 5\% EtOAc/DCM) to afford 18 (536 mg, 54\%), S6 (260 mg, 26\%), and recovered 17 (164 mg, 18\%).

\section{Preparation of vinyllithium:}

THF $(38.0 \mathrm{~mL})$ was added to a flame-dried $200 \mathrm{~mL}$ round-bottom flask under $\mathrm{N}_{2}$, followed by tetravinyl tin $(2.10 \mathrm{~mL}, 11.5 \mathrm{mmol})$. The solution was cooled to $-78{ }^{\circ} \mathrm{C}$ and $n$-BuLi $(17.3 \mathrm{~mL}, 2.5 \mathrm{M}$ in hexanes, $43.3 \mathrm{mmol}, 3.76$ equiv) was added dropwise. The reaction was stirred for 20 minutes at $-78{ }^{\circ} \mathrm{C}$, then lifted out of the ice bath and allowed to warm to room temperature. The reaction was allowed to stir at room temperature for at least 2 hours before use, provides a $\sim 0.756 \mathrm{M}$ solution of vinyllithium (note: highest yields for 1,2-addition are obtained after stirring for 6 hours, at which time the mixture should be slightly milky grey in appearance). 
Data for 18 (major diastereomer, peak 2): $[\alpha]_{D}^{25.0}=-23.8^{\circ}\left(\mathrm{c}=1.07, \mathrm{CHCl}_{3}\right)$.

${ }^{1}$ H NMR $\left(400 \mathrm{MHz}, \mathrm{CDCl}_{3}\right) \delta 6.73(\mathrm{~d}, J=2.3 \mathrm{~Hz}, 1 \mathrm{H}), 6.40(\mathrm{~d}, J=2.3 \mathrm{~Hz}, 1 \mathrm{H}), 6.19(\mathrm{dd}, J=17.2,10.6$ $\mathrm{Hz}, 1 \mathrm{H}), 6.04$ (d, $J=1.5 \mathrm{~Hz}, 1 \mathrm{H}), 5.77$ (ddd, $J=17.1,10.2,8.9 \mathrm{~Hz}, 1 \mathrm{H}), 5.46$ (dd, $J=17.3,1.6 \mathrm{~Hz}, 1 \mathrm{H})$, $5.21(\mathrm{dd}, J=10.6,1.5 \mathrm{~Hz}, 1 \mathrm{H}), 4.92$ (dddd, $J=17.0,14.0,2.2,0.8 \mathrm{~Hz}, 2 \mathrm{H}), 3.79$ (s, 3H), 3.74 (s, 3H), $2.40-2.22(\mathrm{~m}, 2 \mathrm{H}), 2.07(\mathrm{q}, J=9.6 \mathrm{~Hz}, 1 \mathrm{H}), 1.92(\mathrm{dt}, J=14.3,4.5 \mathrm{~Hz}, 1 \mathrm{H}), 1.51$ (q, $J=14.0,13.3 \mathrm{~Hz}$, 2H), $1.44(\mathrm{~d}, J=12.7 \mathrm{~Hz}, 1 \mathrm{H}), 1.41(\mathrm{~d}, J=9.4 \mathrm{~Hz}, 2 \mathrm{H}), 1.35-1.17(\mathrm{~m}, 2 \mathrm{H}), 1.12(\mathrm{~s}, 3 \mathrm{H}), 0.97$ (d, $J=1.2$ $\mathrm{Hz}, 6 \mathrm{H})$.

${ }^{13}$ C NMR (101 MHz, $\left.\mathrm{CDCl}_{3}\right) \delta 159.7,158.3,146.6,145.3,140.5,125.3,120.4,119.7,114.8,112.9$, $108.5,98.1,76.1,55.8,55.7,49.3,47.0,46.1,34.7,34.6,34.4,33.1,30.0,23.8,23.4,22.9$.

FTIR ( $\mathrm{NaCl}$, thin film) 3424 (br) 3001, 2950, 2930, 2858, 2832, 1599, 1560, 1483, 1459, 1434, 1406, $1379,1366,1301,1268,1210,1145,1053,1037,994,910,879,830,811 \mathrm{~cm}^{-1}$

HRMS (MM) calc'd for $\mathrm{C}_{26} \mathrm{H}_{34} \mathrm{BrO}_{2}[\mathrm{M}-\mathrm{OH}]^{+}$457.1742, found 457.1744.

Data for $\mathbf{S 6}$ (minor diastereomer, peak 1): $[\alpha]_{D}^{25.0}=-34.2^{\circ}\left(\mathrm{c}=1.03, \mathrm{CHCl}_{3}\right)$.

${ }^{1}$ H NMR $\left(400 \mathrm{MHz}, \mathrm{CDCl}_{3}\right) \delta 6.75(\mathrm{~d}, J=2.4 \mathrm{~Hz}, 1 \mathrm{H}), 6.42(\mathrm{~d}, J=2.3 \mathrm{~Hz}, 1 \mathrm{H}), 6.23(\mathrm{~d}, J=1.4 \mathrm{~Hz}, 1 \mathrm{H})$, $6.09(\mathrm{dd}, J=17.4,10.3 \mathrm{~Hz}, 1 \mathrm{H}), 5.77$ (ddd, $J=17.2,10.2,8.7 \mathrm{~Hz}, 1 \mathrm{H}), 5.48$ (dd, $J=17.4,1.5 \mathrm{~Hz}, 1 \mathrm{H})$, $5.16(\mathrm{dd}, J=10.3,1.5 \mathrm{~Hz}, 1 \mathrm{H}), 4.93$ (dddd, $J=18.0,15.2,2.2,0.8 \mathrm{~Hz}, 2 \mathrm{H}), 3.79$ (s, 3H), 3.74 (s, 3H), $2.35(\mathrm{t}, J=9.3 \mathrm{~Hz}, 1 \mathrm{H}), 2.15-2.02(\mathrm{~m}, 2 \mathrm{H}), 1.90(\mathrm{dddd}, J=14.3,12.8,4.4,1.6 \mathrm{~Hz}, 1 \mathrm{H}), 1.77-1.65(\mathrm{~m}$, 2H), $1.59(\mathrm{~d}, J=13.2 \mathrm{~Hz}, 1 \mathrm{H}), 1.49-1.41(\mathrm{~m}, 2 \mathrm{H}), 1.34(\mathrm{td}, J=12.8,4.3 \mathrm{~Hz}, 1 \mathrm{H}), 1.11(\mathrm{dtd}, J=12.6$, 4.0, $1.9 \mathrm{~Hz}, 1 \mathrm{H}), 0.97(\mathrm{~d}, J=1.1 \mathrm{~Hz}, 6 \mathrm{H}), 0.89(\mathrm{~s}, 3 \mathrm{H})$.

${ }^{13}$ C NMR (101 MHz, $\left.\mathrm{CDCl}_{3}\right) \delta 159.6,158.4,146.2,144.9,140.6,125.4,120.9,117.9,115.6,114.8$, $108.4,98.1,75.8,55.8,55.7,49.6,49.1,46.5,35.6,34.9,34.5,33.1,30.1,25.0,23.8,22.3$.

FTIR ( $\mathrm{NaCl}$, thin film) 3451 (br) 3073, 2998, 2951, 2934, 2858, 1630, 1560, 1560, 1482, 1461, 1434, $1406,1380,1366,1301,1266,1211,1150,1038,996,936,909,884,830,813 \mathrm{~cm}^{-1}$

HRMS (MM) calc'd for $\mathrm{C}_{26} \mathrm{H}_{34} \mathrm{BrO}_{2}[\mathrm{M}-\mathrm{OH}]^{+}$457.1742, found 457.1744. 


\section{Preparation of aryl bromide 3.}

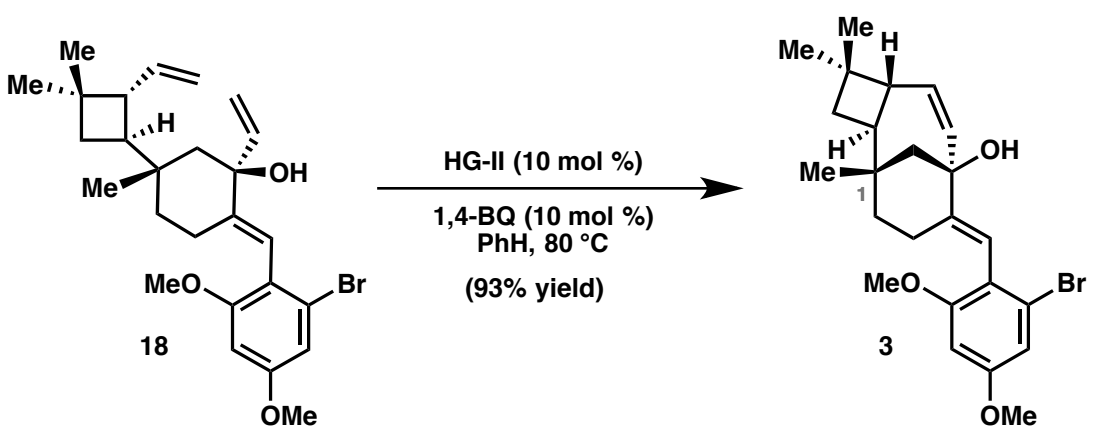

A $250 \mathrm{~mL}$ round-bottom flask containing allylic alcohol $18(807 \mathrm{mg}, 1.70 \mathrm{mmol})$ was pumped into a $\mathrm{N}_{2}$-filled glovebox where Hoveyda-Grubbs second-generation catalyst (106 mg, $0.170 \mathrm{mmol}, 0.100$ equiv) and 1,4-benzoquinone (18.4 mg, $0.170 \mathrm{mmol}, 0.100$ equiv) were added. The flask was sealed under nitrogen, removed from the glovebox and dry benzene $(85.0 \mathrm{~mL})$ was added via syringe. The green reaction mixture was heated to $80{ }^{\circ} \mathrm{C}$ for 12 hours, then cooled to room temperature. Ethyl vinyl ether was added to inactivate the catalyst and stirred for 15 minutes before the reaction mixture was concentrated in vacuo. The crude residue was purified by silica gel flash chromatography (20-30\% $\mathrm{Et}_{2} \mathrm{O} /$ hexane) to afford aryl bromide $3(704 \mathrm{mg}, 93 \%)$ as a white foam and a single diastereomer at $\mathrm{C} 1$ : $[\alpha]_{D}^{25.0}=+95.5^{\circ}\left(\mathrm{c}=0.815, \mathrm{CHCl}_{3}\right)$.

${ }^{1}$ H NMR $\left(400 \mathrm{MHz}, \mathrm{CDCl}_{3}\right) \delta 6.74(\mathrm{~d}, J=2.4 \mathrm{~Hz}, 1 \mathrm{H}), 6.41(\mathrm{~d}, J=2.4 \mathrm{~Hz}, 1 \mathrm{H}), 6.32(\mathrm{~d}, J=1.7 \mathrm{~Hz}, 1 \mathrm{H})$, $5.84(\mathrm{dd}, J=10.8,2.4 \mathrm{~Hz}, 1 \mathrm{H}), 5.34(\mathrm{ddd}, J=10.8,2.8,2.1 \mathrm{~Hz}, 1 \mathrm{H}), 3.79$ (s, 3H), 3.74 (s, 3H), 2.53 (dt, $J$ $=11.6,2.6 \mathrm{~Hz}, 1 \mathrm{H}), 2.37(\mathrm{td}, J=11.2,7.9 \mathrm{~Hz}, 1 \mathrm{H}), 2.28(\mathrm{dd}, J=12.8,2.3 \mathrm{~Hz}, 1 \mathrm{H}), 2.18$ (dddd, $J=15.1$, 11.0, 6.0, 1.9 Hz, 1H), 2.08 (ddd, $J=15.1,5.2,3.6 \mathrm{~Hz}, 1 \mathrm{H}), 1.75(\mathrm{~s}, 1 \mathrm{H}), 1.60-1.46(\mathrm{~m}, 2 \mathrm{H}), 1.41(\mathrm{dd}, J$ $=12.9,2.2 \mathrm{~Hz}, 1 \mathrm{H}), 1.37-1.22(\mathrm{~m}, 2 \mathrm{H}), 1.08(\mathrm{~s}, 3 \mathrm{H}), 1.06(\mathrm{~s}, 3 \mathrm{H}), 0.90(\mathrm{~s}, 3 \mathrm{H})$.

${ }^{13}$ C NMR $\left(101 \mathrm{MHz}, \mathrm{CDCl}_{3}\right) \delta 159.6,158.5,147.7,134.6,131.6,125.5,120.8,116.3,108.5,98.2,76.7$, $55.9,55.7,50.9,45.4,43.4,38.3,37.5,35.1,32.3,30.9,26.2,24.5,21.5$.

FTIR (NaCl, thin film) 3422 (br) 3002, 2949, 2930, 2862, 1599, 1562, 1481, 1462, 1455, 1434, 1405, $1366,1302,1267,1211,1149,1037,1015,979,938,870,858,830,813,772,755 \mathrm{~cm}^{-1}$

HRMS (MM) calc'd for $\mathrm{C}_{24} \mathrm{H}_{30} \mathrm{BrO}_{2}[\mathrm{M}-\mathrm{OH}]^{+}$429.1429, found 429.1429. 


\section{Preparation of tertiary alcohols 19 and S7.}
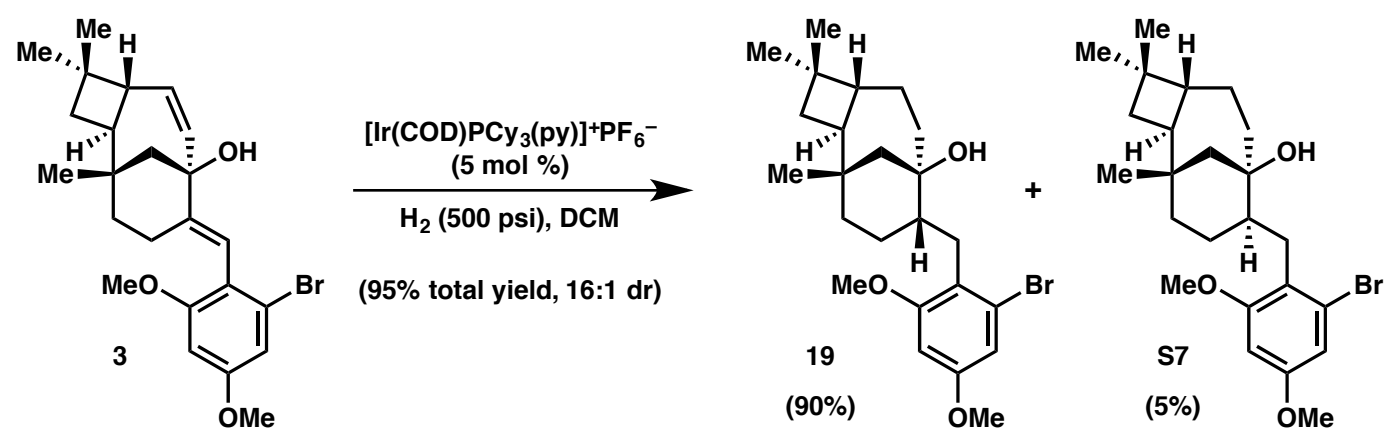

Inside a $\mathrm{N}_{2}$-filled glovebox, Crabtree's catalyst $(59.6 \mathrm{mg}, 0.074 \mathrm{mmol}$, 0.05 equiv) was added to a $100 \mathrm{~mL}$ round-bottom flask containing bromide 3 (663 mg, $1.48 \mathrm{mmol})$. DCM (14.8 mL) was added and the flask was placed inside a steel bomb, which was closed under nitrogen and brought out of the glovebox. The pressure gauge was quickly attached and all bolts on the bomb tightened with a wrench. The bomb was connected to a $\mathrm{H}_{2}$ inlet and the vessel purged with 250 psi $\mathrm{H}_{2}$ three times before being charged to 500 psi. The reaction was stirred at room temperature for 3 hours, at which time $\mathrm{H}_{2}$ was vented from the reaction. DCM was removed in vacuo and the crude residue purified by silica gel flash chromatography (isocratic: $40 \% \mathrm{Et}_{2} \mathrm{O} /$ hexane) to afford tertiary alcohols 19 (599 $\mathrm{mg}, 90 \%)$ and $\mathbf{S 7}(37.4 \mathrm{mg}, 5 \%)$ as white, crystalline solids.

Data for 19 (major diastereomer, peak 2): $[\alpha]_{D}^{25.0}=-20.5^{\circ}\left(\mathrm{c}=0.900, \mathrm{CHCl}_{3}\right)$. ${ }^{1} \mathbf{H}$ NMR $\left(400 \mathrm{MHz}, \mathrm{CDCl}_{3}\right) \delta 6.70(\mathrm{~d}, J=2.4 \mathrm{~Hz}, 1 \mathrm{H}), 6.40(\mathrm{~d}, J=2.4 \mathrm{~Hz}$,

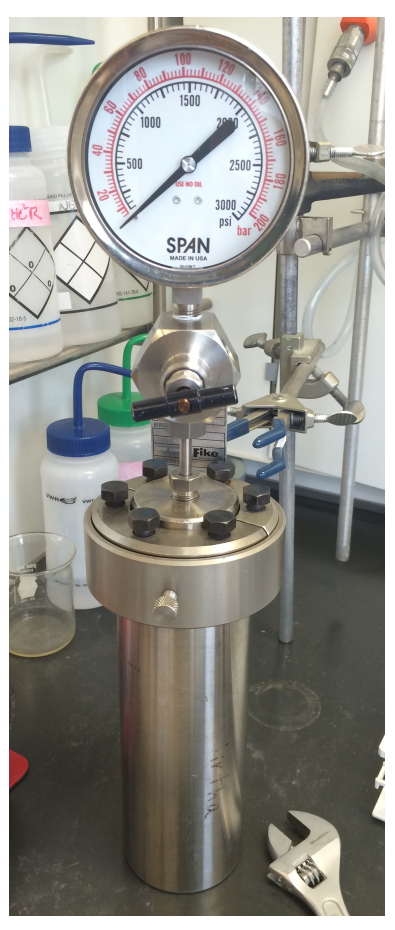
$1 \mathrm{H}), 3.79$ (s, 3H), 3.77 (s, 3H), $3.03(\mathrm{dd}, J=13.2,2.7 \mathrm{~Hz}, 1 \mathrm{H}), 2.61(\mathrm{dd}, J=13.2,10.0 \mathrm{~Hz}, 1 \mathrm{H}), 2.08$ (ddd, $J=11.9,10.6,7.9 \mathrm{~Hz}, 1 \mathrm{H}), 1.95(\mathrm{ddd}, J=13.8,10.4,3.6 \mathrm{~Hz}, 1 \mathrm{H}), 1.83(\mathrm{dd}, J=12.8,2.6 \mathrm{~Hz}, 1 \mathrm{H})$, $1.78-1.57(\mathrm{~m}, 4 \mathrm{H}), 1.57-1.34(\mathrm{~m}, 5 \mathrm{H}), 1.34-1.23(\mathrm{~m}, 2 \mathrm{H}), 1.01(\mathrm{~s}, 3 \mathrm{H}), 0.99(\mathrm{~s}, 3 \mathrm{H}), 0.96(\mathrm{dd}, J=$ $12.9,5.6 \mathrm{~Hz}, 2 \mathrm{H}), 0.77$ (s, 3H).

${ }^{13}$ C NMR $\left(101 \mathrm{MHz}, \mathrm{CDCl}_{3}\right) \delta 158.9,158.9,125.9,122.8,108.9,98.4,76.2,55.9,55.6,51.7,50.1,45.4$, $38.7,38.0,36.0,35.0,33.9,33.3,30.8,28.5,26.6,26.3,21.3,21.0$.

FTIR (NaCl, thin film) 3474 (br), 3000, 2946, 2930, 2862, 1603, 1568, 1482, 1461, 1435, 1410, 1294, $1272,1212,1198,1151,1130,1054,1038,999,937,926,876,831,756 \mathrm{~cm}^{-1}$

HRMS (MM) calc'd for $\mathrm{C}_{24} \mathrm{H}_{34} \mathrm{BrO}_{2}[\mathrm{M}-\mathrm{OH}]^{+} 433.1737$, found 433.1685 . 
Data for $\mathbf{S} 7$ (minor diastereomer, peak 1): $[\alpha]_{D}^{25.0}=-27.7^{\circ}\left(\mathrm{c}=0.950, \mathrm{CHCl}_{3}\right)$.

${ }^{1}$ H NMR $\left(400 \mathrm{MHz}, \mathrm{CDCl}_{3}\right) \delta 6.72(\mathrm{~d}, J=2.5 \mathrm{~Hz}, 1 \mathrm{H}), 6.40(\mathrm{~d}, J=2.4 \mathrm{~Hz}, 1 \mathrm{H}), 3.80(\mathrm{~s}, 3 \mathrm{H}), 3.78$ (s, 3H), $2.85(\mathrm{dd}, J=13.6,5.8 \mathrm{~Hz}, 1 \mathrm{H}), 2.59$ (dd, $J=13.6,8.1 \mathrm{~Hz}, 1 \mathrm{H}), 2.29$ (dddd, $J=11.4,8.0,5.8,3.5$ $\mathrm{Hz}, 1 \mathrm{H}), 1.98$ (ddd, $J=11.0,9.4,6.7 \mathrm{~Hz}, 2 \mathrm{H}), 1.80-1.52(\mathrm{~m}, 4 \mathrm{H}), 1.52-1.37$ (m, 4H), 1.37 - 1.26 (m, 4H), $1.25(\mathrm{~s}, 1 \mathrm{H}), 0.94(\mathrm{~s}, 6 \mathrm{H}), 0.78(\mathrm{~s}, 3 \mathrm{H})$.

${ }^{13}$ C NMR $\left(101 \mathrm{MHz}, \mathrm{CDCl}_{3}\right) \delta 158.9,158.5,126.1,123.0,109.2,98.3,75.2,55.8,55.7,49.3,46.4,45.4$, $41.0,38.5,36.9,36.6,33.8,30.5,30.4,29.2,27.6,24.2,24.1,21.5$.

FTIR ( $\mathrm{NaCl}$, thin film) 3482 (br), 2998, 2945, 2928, 2859, 1690, 1648, 1602, 1567, 1482, 1459, 1435, 1409, 1381, 1364, 1294, 1273, 1211, 1198, 1154, 1134, 1051, 1039, 973, 937, 830, 809, 756.

HRMS (MM) calc'd for $\mathrm{C}_{24} \mathrm{H}_{35} \mathrm{BrO}_{3} \mathrm{Na}[\mathrm{M}+\mathrm{Na}]^{+}$475.1818, found 475.1858.

\section{Preparation of pentacycle 2.}

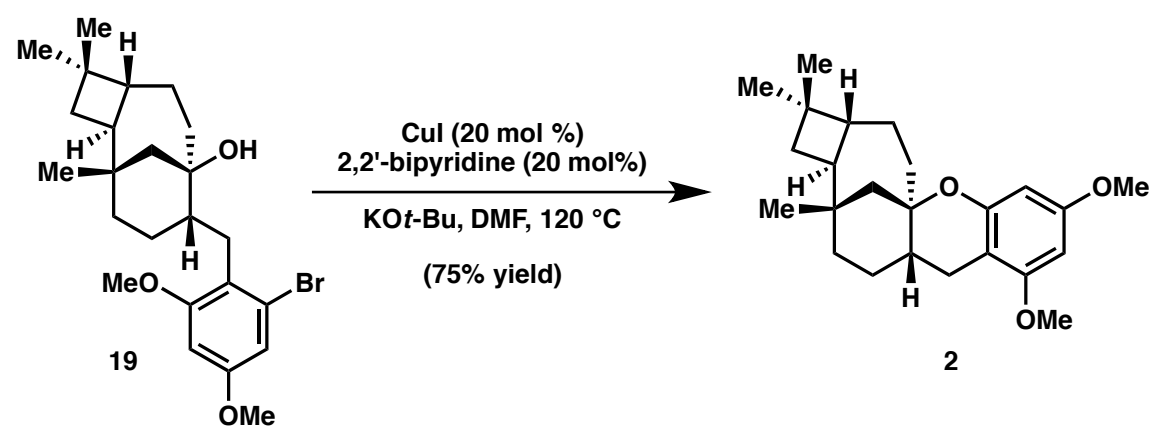

Aryl bromide 19 (274 mg, $0.607 \mathrm{mmol})$ was added to each of two $20 \mathrm{~mL}$ scintillation vials and pumped inside a $\mathrm{N}_{2}$-filled glovebox, where CuI (23.1 mg, $0.121 \mathrm{mmol}, 0.200$ equiv), 2,2'-bipyridine (18.9, $0.121 \mathrm{mmol}, 0.200$ equiv), and $\mathrm{KO} t$-Bu (204, $1.82 \mathrm{mmol}, 3.00$ equiv) were added as solids to each. Dry DMF $(6.10 \mathrm{~mL})$ was then added, the reaction sealed under $\mathrm{N}_{2}$ with a Teflon screw-cap and heated to $120{ }^{\circ} \mathrm{C}$ in a pre-heated aluminum block inside the glovebox for 3.5 hours. After cooling to room temperature, the reaction mixtures were combined and loaded directly onto a short silica gel column, preequilibrated with $5 \% \mathrm{Et}_{2} \mathrm{O} /$ hexane. The column was eluted with $5 \% \mathrm{Et}_{2} \mathrm{O} /$ hexane (isocratic) to afford pentacycle $2(339 \mathrm{mg}, 75 \%)$ as a white solid: $[\alpha]_{D}^{25.0}=+42.4^{\circ}\left(\mathrm{c}=1.08, \mathrm{CHCl}_{3}\right)$.

${ }^{1}$ H NMR $\left(400 \mathrm{MHz}, \mathrm{CDCl}_{3}\right) \delta 6.02(\mathrm{~d}, J=2.4 \mathrm{~Hz}, 1 \mathrm{H}), 6.00(\mathrm{~d}, J=2.4 \mathrm{~Hz}, 1 \mathrm{H}), 3.77(\mathrm{~s}, 3 \mathrm{H}), 3.74(\mathrm{~s}$, $3 \mathrm{H}), 2.55(\mathrm{dd}, J=16.6,4.0 \mathrm{~Hz}, 1 \mathrm{H}), 2.20-2.03(\mathrm{~m}, 2 \mathrm{H}), 1.97(\mathrm{dd}, J=12.6,2.3 \mathrm{~Hz}, 1 \mathrm{H}), 1.77$ (dddd, $J=$ 18.7, 9.0, 7.4, 4.8 Hz, 2H), $1.69-1.55(\mathrm{~m}, 4 \mathrm{H}), 1.52-1.41(\mathrm{~m}, 3 \mathrm{H}), 1.32(\mathrm{t}, J=10.7,9.6 \mathrm{~Hz}, 2 \mathrm{H}), 1.32-$ $1.20(\mathrm{~m}, 1 \mathrm{H}), 1.17(\mathrm{dd}, J=12.6,1.1 \mathrm{~Hz}, 1 \mathrm{H}), 0.99(\mathrm{~s}, 3 \mathrm{H}), 0.97$ (s, 3H), $0.86(\mathrm{~s}, 3 \mathrm{H})$. 
${ }^{13}$ C NMR $\left(101 \mathrm{MHz}, \mathrm{CDCl}_{3}\right) \delta 159.5,158.3,154.4,104.5,94.2,90.8,79.9,55.5,55.4,48.0,44.3,40.9$, $38.0,36.9,35.6,35.1,33.5,30.8,28.4,27.1,26.5,22.8,20.9,19.9$.

FTIR ( $\mathrm{NaCl}$, thin film) 2995, 2945, 2928, 2862, 2843, 1617, 1589, 1494, 1460, 1420, 1363, 1288, 1215, $1201,1186,1164,1145,1108,1074,1054,1033,1008,942,928,810 \mathrm{~cm}^{-1}$

HRMS (MM) calc'd for $\mathrm{C}_{24} \mathrm{H}_{35} \mathrm{O}_{3}[\mathrm{M}+\mathrm{H}]^{+} 371.2581$, found 371.2578 .

Preparation of benzylic ethers 20 and $\mathbf{S 8} .^{10}$

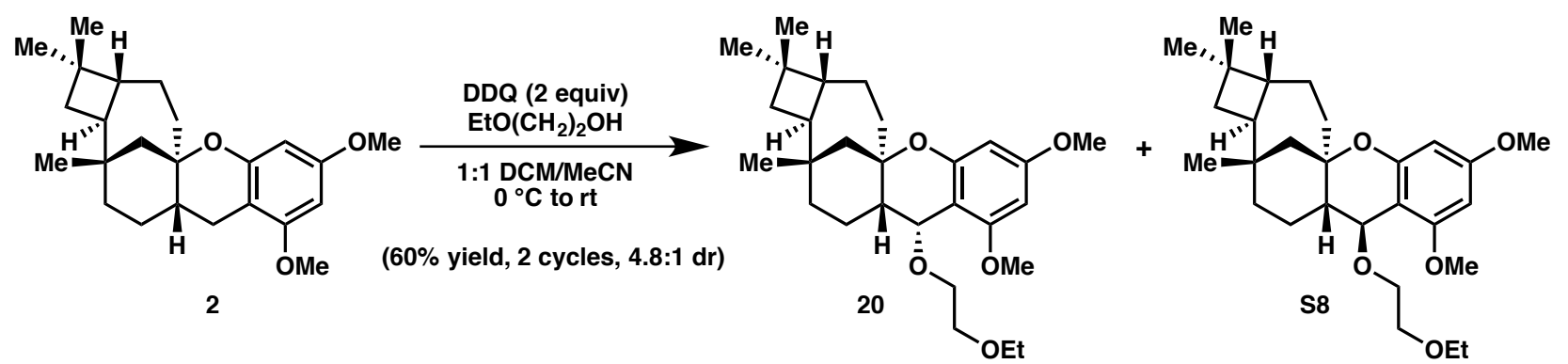

To a flame-dried $25 \mathrm{~mL}$ round-bottom flask was added pentacycle $2(80.0 \mathrm{mg}, 0.216 \mathrm{mmol})$ and the atmosphere exchanged three times for argon. A 1:1 mixture of dry MeCN/DCM $(6.40 \mathrm{~mL})$ was then added, followed by ethoxyethanol $(1.54 \mathrm{~mL})$ via syringe and the solution cooled to $0{ }^{\circ} \mathrm{C}$. A previously prepared stock solution of DDQ in dry $\mathrm{MeCN}(0.860 \mathrm{~mL}, 0.508 \mathrm{M}, 2.00$ equiv) was then added dropwise. The reaction turned grey/blue immediately upon addition of DDQ and slowly turned green-blue by the end of addition. Once the addition was complete, the reaction was lifted from the ice bath and gradually warmed to room temperature. The color became an olive green-brown after 1 hour, indicating the reaction had stalled at $\sim 50 \%$ conversion (as judged by TLC). At this point, the reaction was quenched with a saturated solution of aqueous $\mathrm{NaHCO}_{3}$ and stirred vigorously for 10 minutes before the layers were separated. The aqueous layer was extracted twice more with DCM and the combined organic layers were washed with one portion of DI $\mathrm{H}_{2} \mathrm{O}$, dried over $\mathrm{Na}_{2} \mathrm{SO}_{4}$, filtered and concentrated in vacuo. The crude residue was purified by silica gel flash chromatography: $\mathrm{SiO}_{2}$ was first deactivated by applying a few drops of aqueous $\mathrm{NH}_{4} \mathrm{OH}(28 \%)$ to the top of a dry column and equilibrating with $100 \mathrm{~mL}$ of $5 \%$ $\mathrm{Et}_{2} \mathrm{O} /$ hexane. The crude residue was then applied and eluted with fresh $5 \% \mathrm{Et}_{2} \mathrm{O} /$ hexane until unreacted 2 elutes completely, then $20 \% \mathrm{Et}_{2} \mathrm{O} /$ hexane until complete elution of second diastereomer to afford a mixture of $\mathbf{2 0}$ and $\mathbf{S 8}$ (41.0 $\mathrm{mg}, 41 \%$ yield), and recovered starting material 2 (40.8 $\mathrm{mg}, 51 \%)$. The recovered starting material was re-subjected to the reaction conditions described above to afford additional 20 and S8 (18.8 mg, 60\% total over 2 cycles) and 2 (15.2 mg, 74\% overall brsm). Analytically 
pure samples of $\mathbf{2 0}$ and $\mathbf{S 8}$ were obtained by preparative TLC (30\% $\left.\mathrm{Et}_{2} \mathrm{O}, 1 \% \mathrm{Et}_{3} \mathrm{~N} / \mathrm{hexane}\right)$ and a representative spectrum of the mixture as used in the next step is also provided.

Data for 20 (major diastereomer, peak 1): $[\alpha]_{D}^{25.0}=+43.5^{\circ}\left(\mathrm{c}=0.815, \mathrm{CHCl}_{3}\right)$.

${ }^{1}$ H NMR $\left(400 \mathrm{MHz}, \mathrm{CDCl}_{3}\right) \delta 6.01(\mathrm{~d}, J=2.4 \mathrm{~Hz}, 1 \mathrm{H}), 5.94(\mathrm{~d}, J=2.3 \mathrm{~Hz}, 1 \mathrm{H}), 4.27(\mathrm{~d}, J=3.3 \mathrm{~Hz}, 1 \mathrm{H})$, $3.98(\mathrm{dt}, J=9.8,4.9 \mathrm{~Hz}, 1 \mathrm{H}), 3.81(\mathrm{dd}, J=10.9,5.1 \mathrm{~Hz}, 1 \mathrm{H}), 3.79(\mathrm{~s}, 3 \mathrm{H}), 3.73(\mathrm{~s}, 3 \mathrm{H}), 3.58(\mathrm{dd}, J=5.9$, $4.9 \mathrm{~Hz}, 2 \mathrm{H}), 3.53(\mathrm{q}, J=7.0 \mathrm{~Hz}, 2 \mathrm{H}), 2.48-2.36(\mathrm{~m}, 1 \mathrm{H}), 2.21$ (qd, $J=14.4,4.1 \mathrm{~Hz}, 1 \mathrm{H}), 2.10(\mathrm{ddd}, J=$ $12.2,10.7,7.9 \mathrm{~Hz}, 1 \mathrm{H}), 2.00(\mathrm{dd}, J=12.7,2.3 \mathrm{~Hz}, 1 \mathrm{H}), 1.79-1.61(\mathrm{~m}, 3 \mathrm{H}), 1.59-1.41(\mathrm{~m}, 4 \mathrm{H}), 1.42-$ $1.20(\mathrm{~m}, 4 \mathrm{H}), 1.20$ (t, $J=7.0 \mathrm{~Hz}, 3 \mathrm{H}), 0.99$ (s, 3H), 0.98 (s, 3H), 0.86 (s, 3H).

${ }^{13}$ C NMR $\left(101 \mathrm{MHz}, \mathrm{CDCl}_{3}\right) \delta 161.1,159.7,154.7,106.6,93.8,91.2,80.5,72.1,71.4,70.5,66.7,55.4$, $55.4,49.3,47.6,46.1,39.4,39.3,36.3,34.8,33.5,32.4,30.8,26.8,23.1,22.3,20.8,15.4$.

FTIR ( $\mathrm{NaCl}$, thin film) 2948, 2930, 2864, 1614, 1589, 1491,1462, 1438, 1424, 1365, 1353, 1332, 1320, $1287,1215,1202,1189,1166,1148,1109,1053,1033,1005,951,921,866,811,731,638 \mathrm{~cm}^{-1}$

HRMS (MM) calc'd for $\mathrm{C}_{24} \mathrm{H}_{33} \mathrm{O}_{3}\left[\mathrm{M}-\mathrm{O}\left(\mathrm{CH}_{2}\right)_{2} \mathrm{OEt}\right]^{+}$369.2424, found 369.2430.

Data for S8 (minor diastereomer, peak 2): $[\alpha]_{D}^{25.0}=+29.6^{\circ}\left(\mathrm{c}=0.230, \mathrm{CHCl}_{3}\right)$.

${ }^{1}$ H NMR $\left(400 \mathrm{MHz}, \mathrm{CDCl}_{3}\right) \delta 6.02(\mathrm{~d}, J=2.4 \mathrm{~Hz}, 1 \mathrm{H}), 5.92(\mathrm{~d}, J=2.4 \mathrm{~Hz}, 1 \mathrm{H}), 4.30(\mathrm{~d}, J=10.4 \mathrm{~Hz}$, 1H), $3.78(\mathrm{~s}, 3 \mathrm{H}), 3.75(\mathrm{ddd}, J=9.1,3.0,1.3 \mathrm{~Hz}, 1 \mathrm{H}), 3.73(\mathrm{~s}, 3 \mathrm{H}), 3.58-3.45(\mathrm{~m}, 5 \mathrm{H}), 2.24-2.04(\mathrm{~m}$, 2H), $1.92(\mathrm{ddt}, J=14.7,9.9,3.2 \mathrm{~Hz}, 2 \mathrm{H}), 1.80-1.64(\mathrm{~m}, 2 \mathrm{H}), 1.65-1.40(\mathrm{~m}, 5 \mathrm{H}), 1.36-1.19(\mathrm{~m}, 4 \mathrm{H})$, $1.19(\mathrm{t}, J=7.0 \mathrm{~Hz}, 3 \mathrm{H}), 0.97(\mathrm{~s}, 3 \mathrm{H}), 0.96(\mathrm{~s}, 3 \mathrm{H}), 0.86(\mathrm{~s}, 3 \mathrm{H})$.

${ }^{13}$ C NMR $\left(101 \mathrm{MHz}, \mathrm{CDCl}_{3}\right) \delta 161.0,160.0,155.9,105.8,94.2,91.7,81.7,73.4,70.5,67.8,66.7,55.5$, $55.4,48.2,47.2,44.4,37.8,37.2,35.6,35.1,33.2,30.8,28.4,26.4,23.7,20.9,20.4,15.4$.

FTIR ( $\mathrm{NaCl}$, thin film) 2947, 2934, 2864, 1613, 1587, 1490, 1459, 1438, 1421, 1364, 1349, 1312, 1288, $1267,1245,1216,1202,1147,1107,1054,1034,1002,973,943,868.812,736,636 \mathrm{~cm}^{-1}$

HRMS (MM) calc'd for $\mathrm{C}_{24} \mathrm{H}_{33} \mathrm{O}_{3}\left[\mathrm{M}-\mathrm{O}\left(\mathrm{CH}_{2}\right)_{2} \mathrm{OEt}\right]^{+}$369.2424, found 369.2427. 


\section{Preparation of diarylmethanes 21 and S9.}
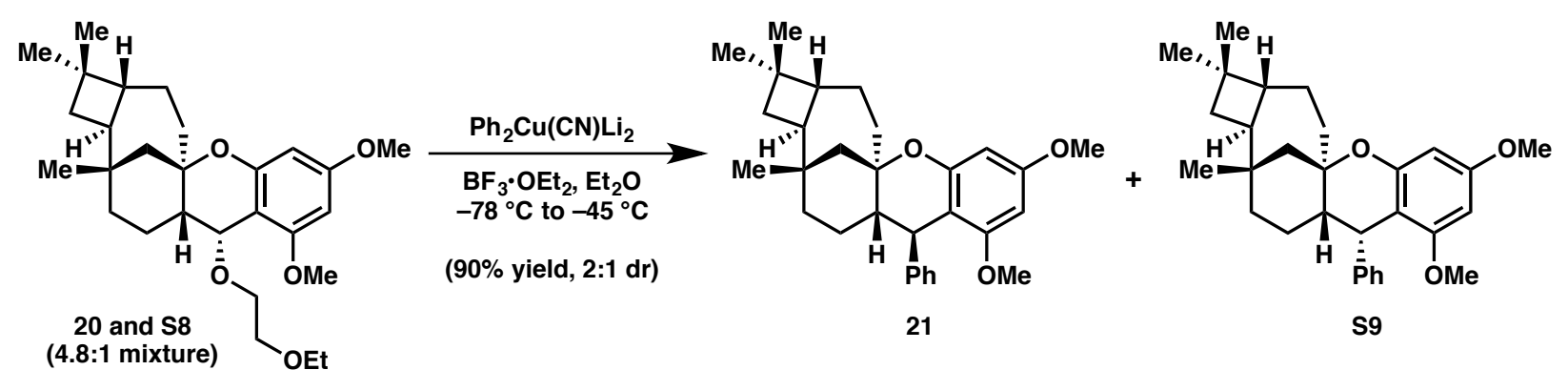

A $10 \mathrm{~mL}$ round-bottom flask containing $\mathrm{CuCN}(11.9 \mathrm{mg}, 0.133 \mathrm{mmol}, 2.05$ equiv) was flamedried under vacuum. After cooling to room temperature, the flask was backfilled with argon and $\operatorname{dry} \mathrm{Et}_{2} \mathrm{O}$ $(2.70 \mathrm{~mL})$ was added via syringe. The suspension was cooled to $-78{ }^{\circ} \mathrm{C}$ under argon and $\mathrm{PhLi}(0.140 \mathrm{~mL}$, 1.9 $\mathrm{M}$ in dibutyl ether, $0.266 \mathrm{mmol}, 4.09$ equiv) was added dropwise. After stirring at $-78{ }^{\circ} \mathrm{C}$ for 5 minutes, the reaction was warmed to $0{ }^{\circ} \mathrm{C}$ and stirred for an additional 30 minutes. The higher-order cuprate was then cooled back to $-78{ }^{\circ} \mathrm{C}$ and the $4.8: 1$ mixture of benzylic ethers $\mathbf{2 0}$ and $\mathbf{S 8}(30.0 \mathrm{mg}$, $0.065 \mathrm{mmol})$ was as a solution in $\mathrm{Et}_{2} \mathrm{O}(1.00 \mathrm{~mL})$. The reaction was stirred for 1-2 minutes before $\mathrm{BF}_{3} \cdot \mathrm{OEt}_{2}(0.160 \mathrm{~mL}, 1.30 \mathrm{mmol}, 20.0$ equiv) was added dropwise via syringe. The reaction was stirred at $-78{ }^{\circ} \mathrm{C}$ for 10 minutes, then quickly transferred to a pre-equilibrated bath at $-55^{\circ} \mathrm{C}$, which was allowed to $-50{ }^{\circ} \mathrm{C}$ over 5 minutes, then maintained at or just below $-45{ }^{\circ} \mathrm{C}$ for another 30 minutes. The reaction was checked for completion by TLC, then quenched with aqueous $\mathrm{NaHCO}_{3}$ and warmed to room temperature. The layers were separated and the aqueous layer extracted twice with $\mathrm{Et}_{2} \mathrm{O}$. The combined organic layers were dried over $\mathrm{MgSO}_{4}$, filtered and concentrated in vacuo. The crude residue was purified by silica gel flash chromatography (isocratic: $5 \% \mathrm{Et}_{2} \mathrm{O} /$ hexane) to afford diarylmethanes $\mathbf{2 1}$ and $\mathbf{S 9}$ (26.3 mg, 90\%) as a 2:1 inseparable mixture, white solid: $[\alpha]_{D}^{25.0}=+2.08^{\circ}\left(\mathrm{c}=1.23, \mathrm{CHCl}_{3}\right)$.

${ }^{1}$ H NMR (2:1 dr, asterisk denotes minor diastereomer, $\left.400 \mathrm{MHz}, \mathrm{CDCl}_{3}\right) \delta 7.24-6.96(\mathrm{~m}, 5 \mathrm{H}), 6.10^{*}(\mathrm{~d}$, $J=2.4 \mathrm{~Hz}, 1 \mathrm{H}), 6.07(\mathrm{~d}, J=2.5 \mathrm{~Hz}, 1 \mathrm{H}), 6.03 *(\mathrm{~d}, J=2.4 \mathrm{~Hz}, 1 \mathrm{H}), 5.93(\mathrm{~d}, J=2.4 \mathrm{~Hz}, 1 \mathrm{H}), 4.10^{*}(\mathrm{~d}, J=$ $6.4 \mathrm{~Hz}, 1 \mathrm{H}), 3.79 *(\mathrm{~s}, 3 \mathrm{H}), 3.76(\mathrm{~s}, 3 \mathrm{H}), 3.46^{*}(\mathrm{~s}, 3 \mathrm{H}), 3.47$ (d, $\left.J=11.3 \mathrm{~Hz}, 1 \mathrm{H}\right), 3.20(\mathrm{~s}, 3 \mathrm{H}), 2.23-2.06$ (m, 1H), $2.00(\mathrm{dd}, J=12.7,2.4 \mathrm{~Hz}, 1 \mathrm{H}), 1.93^{*}(\mathrm{dd}, J=12.6,2.5 \mathrm{~Hz}, 1 \mathrm{H}), 1.88-1.73(\mathrm{~m}, 2 \mathrm{H}), 1.73-$ $1.60(\mathrm{~m}, 3 \mathrm{H}), 1.55-1.41(\mathrm{~m}, 4 \mathrm{H}), 1.39-1.26(\mathrm{~m}, 5 \mathrm{H}), 1.20(\mathrm{q}, J=13.1,12.1 \mathrm{~Hz}, 1 \mathrm{H}), 1.00(\mathrm{~s}, 3 \mathrm{H}), 0.99$ $(\mathrm{s}, 3 \mathrm{H}), 0.92 *(\mathrm{~s}, 3 \mathrm{H}), 0.83(\mathrm{~s}, 3 \mathrm{H}), 0.82 *(\mathrm{~s}, 3 \mathrm{H}), 0.74 *(\mathrm{~s}, 3 \mathrm{H})$.

${ }^{13}$ C NMR (20, major diastereomer, $\left.101 \mathrm{MHz}, \mathrm{CDCl}_{3}\right) \delta$ 160.0, 159.2, 155.2, 146.7, 129.8, 127.7, 125.4, 109.2, 94.5, 92.7, 80.4, 55.3, 55.2, 50.7, 48.1, 44.3, 41.9, 37.9, 36.9, 35.6, 35.1, 33.3, 30.8, 28.2, 26.3, $24.3,20.9,20.2$. 
${ }^{13}$ C NMR (S9, minor diastereomer $101 \mathrm{MHz}, \mathrm{CDCl}_{3}$ ) $\delta 160.1,159.2,155.1,141.5,128.9,127.0,125.5$, $106.4,94.1,91.5,80.7,55.6,55.4,50.0,45.8,44.3,39.3,38.5,36.7,35.5,35.0,33.2,32.7,30.8,26.2$, 25.8, 20.9, 20.4.

FTIR ( $\mathrm{NaCl}$, thin film) 3081, 3059, 3025, 2998, 2948, 2934, 2864, 2843, 1614, 1588, 1490,1460, 1454, 1440, 1420, 1364, 1307, 1288, 1274, 1249, 1216, 1202, 1166, 1148, 1123, 1105, 1076, 1054, 1033, 1005, $943,870,811,759,740,701 \mathrm{~cm}^{-1}$

HRMS (MM) calc'd for $\mathrm{C}_{30} \mathrm{H}_{39} \mathrm{O}_{3}[\mathrm{M}+\mathrm{H}]^{+} 447.2894$, found 447.2905.

\section{Preparation of resorcinols S10 and S11.}

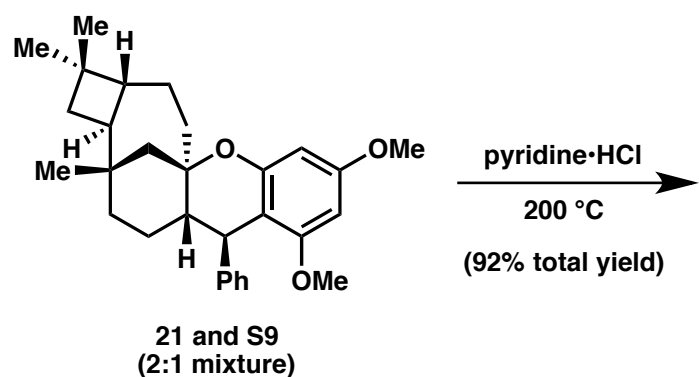

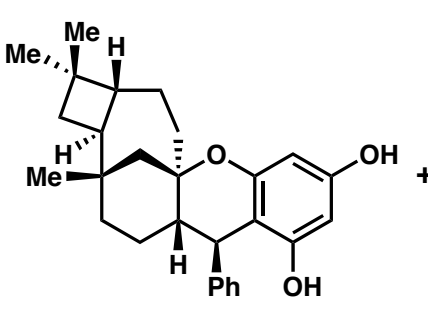

S10

$(62 \%)$

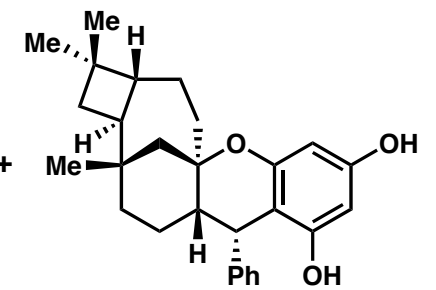

S11

$(30 \%)$

Solid pyridine $\cdot \mathrm{HCl}(1.44 \mathrm{~g}, 12.5 \mathrm{mmol}, 307$ equiv) was weighed into each of two 2-dram vials, containing a 2:1 mixture of diarylmethanes 21 and $\mathbf{S 9}(18.2 \mathrm{mg}, 0.041 \mathrm{mmol})$. The vials were sealed with a Teflon screw-cap under a stream of argon and heated to $200{ }^{\circ} \mathrm{C}$ in a pre-heated aluminum block for 2.5 hours. (Note: it is important to choose a vial/heating block combination that will cover the entire volume of the solid to ensure that it stays completely melted during the course of the reaction). The reactions were cooled to room temperature, during which time the mixture solidified. The crude solids were dissolved in DI $\mathrm{H}_{2} \mathrm{O}$, and combined by pipetting dropwise into an Erlenmeyer flask containing a saturated solution of aqueous $\mathrm{NaHCO}_{3}$. EtOAc was then added and the layers were separated. The aqueous layer was extracted three times with EtOAc and the combined organic layers were dried over $\mathrm{MgSO}_{4}$ and concentrated in vacuo. The crude residue was purified by silica gel flash chromatography (isocratic: 10\% EtOAc, 1\% $\mathrm{AcOH} /$ hexane) to separate catechols $\mathbf{S 1 0}$ and S11. The concentrated fractions for each diastereomer (initially a pale orange oil) were each passed through another short plug of silica gel (eluting with 20\% EtOAc/heaxnes) to remove residual $\mathrm{AcOH}$ and remaining trace impurities to afford $\mathbf{S 1 0}(21.3 \mathrm{mg}, 62 \%)$ and $\mathbf{S 1 1}(10.3 \mathrm{mg}, 30 \%)$ as white solids. 
Data for $\mathbf{S 1 0}$ (major diastereomer, peak 1): $[\alpha]_{D}^{25.0}=-28.2^{\circ}\left(\mathrm{c}=0.475, \mathrm{CHCl}_{3}\right)$.

${ }^{1}$ H NMR $\left(400 \mathrm{MHz}, \mathrm{CDCl}_{3}\right) \delta 7.44-7.26(\mathrm{~m}, 5 \mathrm{H}), 6.00(\mathrm{~d}, J=2.6 \mathrm{~Hz}, 1 \mathrm{H}), 5.88(\mathrm{~d}, J=2.6 \mathrm{~Hz}, 1 \mathrm{H})$, $4.73(\mathrm{~s}, 1 \mathrm{H}), 4.46(\mathrm{~s}, 1 \mathrm{H}), 3.49(\mathrm{~d}, J=11.4 \mathrm{~Hz}, 1 \mathrm{H}), 2.16(\mathrm{ddd}, J=12.3,10.4,7.9 \mathrm{~Hz}, 1 \mathrm{H}), 2.01(\mathrm{dd}, J=$ 12.8, $2.3 \mathrm{~Hz}, 1 \mathrm{H}), 1.85-1.58(\mathrm{~m}, 4 \mathrm{H}), 1.54-1.44$ (m, 2H), $1.44-1.30$ (m, 4H), $1.28(\mathrm{~m}, 1 \mathrm{H}), 1.18$ (d, $J$ $=12.9 \mathrm{~Hz}, 1 \mathrm{H}), 1.10-1.03(\mathrm{~m}, 1 \mathrm{H}), 1.00(\mathrm{~s}, 3 \mathrm{H}), 0.99(\mathrm{~s}, 3 \mathrm{H}), 0.85(\mathrm{~s}, 3 \mathrm{H})$.

${ }^{13}$ C NMR (101 MHz, $\left.\mathrm{CDCl}_{3}\right) \delta 156.1,155.8,155.4,142.3,128.0,106.3,97.7,96.8,80.1,50.5,48.1$, $44.2,41.8,37.8,36.9,35.6,35.2,33.3,30.8,28.6,26.3,24.0,20.9,20.0$.

FTIR ( $\mathrm{NaCl}$, thin film) 3511 (br), 3386 (br), 3060, 3024, 2948, 2928, 2863, 1702, 1627, 1598, 1509, 1492, 1459, 1364, 1349, 1320, 1272, 1248, 1228, 1166, 1138, 1087, 1072, 1057, 1034, 1014, 925, 869, $831,761,738,703,667,638,571,516 \mathrm{~cm}^{-1}$

HRMS (MM) calc'd for $\mathrm{C}_{28} \mathrm{H}_{35} \mathrm{O}_{3}[\mathrm{M}+\mathrm{H}]^{+} 419.2581$, found 419.2591 .

Data for S11 (minor diastereomer, peak 2): $[\alpha]_{D}^{25.0}=+26.7^{\circ}\left(\mathrm{c}=0.180, \mathrm{CHCl}_{3}\right)$.

${ }^{1} \mathbf{H}$ NMR $\left(400 \mathrm{MHz}, \mathrm{CDCl}_{3}\right) \delta 7.37-7.21(\mathrm{~m}, 5 \mathrm{H}), 6.02(\mathrm{~d}, J=2.5 \mathrm{~Hz}, 1 \mathrm{H}), 5.96(\mathrm{~d}, J=2.5 \mathrm{~Hz}, 1 \mathrm{H})$, $4.73(\mathrm{~s}, 1 \mathrm{H}), 4.30(\mathrm{~s}, 1 \mathrm{H}), 4.00(\mathrm{~d}, J=7.0 \mathrm{~Hz}, 1 \mathrm{H}), 2.16(\mathrm{ddd}, J=12.5,7.0,3.9 \mathrm{~Hz}, 1 \mathrm{H}), 1.93(\mathrm{dd}, J=$ 12.7, $2.3 \mathrm{~Hz}, 2 \mathrm{H}), 1.79$ (ddd, $J=12.3,10.3,7.8 \mathrm{~Hz}, 1 \mathrm{H}), 1.66$ (ddd, $J=12.4,8.7,5.4 \mathrm{~Hz}, 1 \mathrm{H}), 1.57$ $1.43(\mathrm{~m}, 5 \mathrm{H}), 1.39-1.28(\mathrm{~m}, 4 \mathrm{H}), 1.23-1.12(\mathrm{~m}, 3 \mathrm{H}), 1.09-0.95(\mathrm{~m}, 2 \mathrm{H}), 0.91(\mathrm{~s}, 3 \mathrm{H}), 0.82(\mathrm{~s}, 3 \mathrm{H})$, $0.75(\mathrm{~s}, 3 \mathrm{H})$.

${ }^{13}$ C NMR (101 MHz, $\left.\mathrm{CDCl}_{3}\right) \delta 156.2,155.7,155.4,138.6,127.4,104.8,97.6,95.9,80.4,49.7,45.6$, $44.2,39.6,38.3,36.6,35.4,35.1,33.2,32.0,30.8,29.9,26.1,25.4,20.8,20.3$.

FTIR $(\mathrm{NaCl}$, thin film) 3385 (br), 3027, 2949, 2925, 2857, 1624, 1600, 1508, 1493, 1459, 1452, 1377, $1364,1247,1190,1163,1143,1086,1055,1034,1015,925,826,761,721,701 \mathrm{~cm}^{-1}$

HRMS (MM) calc'd for $\mathrm{C}_{28} \mathrm{H}_{35} \mathrm{O}_{3}[\mathrm{M}+\mathrm{H}]^{+} 419.2581,419.2595$. 
Preparation of (+)-psiguadial B (1).
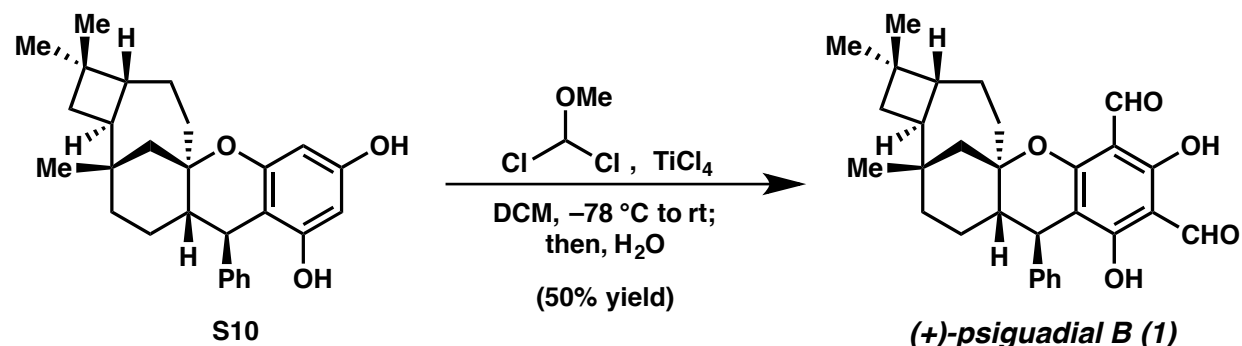

(+)-psiguadial B (1)

HepG2 $I C_{50}=46 \mathrm{nM}$

To a 2-dram vial was added catechol $\mathbf{S 1 0}(15.4 \mathrm{mg}, 0.037 \mathrm{mmol})$ and the atmosphere exchanged three times for $\mathrm{N}_{2}$. DCM $(1.30 \mathrm{~mL})$ was then added via syringe, followed by dichloromethyl methyl ether ( $0.083 \mathrm{~mL}, 0.920 \mathrm{mmol}, 25.0$ equiv). The solution was cooled to $-78{ }^{\circ} \mathrm{C}$ and a freshly prepared stock solution of $\mathrm{TiCl}_{4}(0.190 \mathrm{~mL}, 0.912 \mathrm{M}$ in DCM, $0.173 \mathrm{mmol}, 4.68$ equiv) was added dropwise. The reaction immediately turns dark red. The reaction was stirred at $-78{ }^{\circ} \mathrm{C}$ for 5 minutes, then warmed to room temperature and stirred for an additional 3 hours and 40 minutes. DI $\mathrm{H}_{2} \mathrm{O}(2.00 \mathrm{~mL})$ was then added via syringe and the reaction stirred vigorously for 15 minutes before the layers were separated. The aqueous layer was extracted five times with DCM and the combined organic layers were filtered over a plug of $\mathrm{Na}_{2} \mathrm{SO}_{4}$ and concentrated in vacuo. The crude residue was purified by silica gel flash chromatography (isocratic: 2\% EtOAc, 1\% AcOH/hexane) to afford (+)-psiguadial B (1) (8.7 mg, 50\%) as an ivory solid. Note: $\mathbf{1}$ is streaky on $\mathrm{SiO}_{2}$ and after an initial concentrated band elutes, approximately $12 \%$ of the product is contained in the following very dilute fractions. The natural product is weakly UV active, but can also be visualized by TLC using 2,4-dinitrophenylhydrazine stain.

$[\alpha]_{D}^{25.0}=+94.0^{\circ}\left(\mathrm{c}=0.265, \mathrm{CHCl}_{3}\right)$.

${ }^{1}$ H NMR $\left(400 \mathrm{MHz}, \mathrm{CDCl}_{3}\right) \delta 13.51(\mathrm{~s}, 1 \mathrm{H}), 13.04(\mathrm{~s}, 1 \mathrm{H}), 10.07(\mathrm{~s}, 2 \mathrm{H}), 7.26(\mathrm{dd}, J=14.6,1.5 \mathrm{~Hz}, 2 \mathrm{H})$, $7.23-7.17$ (m, 1H), 7.10 (br s, 2H), 3.49 (d, $J=11.5 \mathrm{~Hz}, 1 \mathrm{H}), 2.20-2.12$ (m, 1H), 2.09 (dd, $J=12.7$, $2.4 \mathrm{~Hz}, 1 \mathrm{H}), 1.92(\mathrm{ddd}, J=14.9,12.8,4.2 \mathrm{~Hz}, 1 \mathrm{H}), 1.82(\mathrm{ddd}, J=12.3,8.8,5.6 \mathrm{~Hz}, 1 \mathrm{H}), 1.73-1.59$ (m, $3 \mathrm{H}), 1.53-1.44(\mathrm{~m}, 1 \mathrm{H}), 1.49(\mathrm{ddd}, J=11.6,8.1,2.9 \mathrm{~Hz}, 2 \mathrm{H}), 1.44-1.29$ (m, 4H), 1.05 (dd, $J=7.6,5.8$ $\mathrm{Hz}, 1 \mathrm{H}), 1.02(\mathrm{~s}, 3 \mathrm{H}), 1.00(\mathrm{~s}, 3 \mathrm{H}), 0.85$ (s, 3H).

${ }^{13}$ C NMR $\left(101 \mathrm{MHz}, \mathrm{CDCl}_{3}\right) \delta 192.3,191.5,169.6,168.5,163.5,143.4,128.2,126.2,105.7,104.6$, 104.1, 84.1, 50.0, 47.4, 44.0, 40.4, 37.6, 36.9, 35.4, 35.1, 33.4, 30.6, 29.3, 26.1, 23.9, 20.7, 20.1.

FTIR ( $\mathrm{NaCl}$, thin film) 3026, 2945, 2926, 2864, 2720, 1633, 1603, 1493, 1437, 1382, 1363, 1300, 1270, $1251,1231,1184,1154,1143,1031,1006,976,926,917,875,851,840,824,768,701,636,618,606$, $564 \mathrm{~cm}^{-1}$

HRMS (MM) calc'd for $\mathrm{C}_{30} \mathrm{H}_{35} \mathrm{O}_{5}[\mathrm{M}+\mathrm{H}]^{+}$475.2479, found 475.2487. 
Comparison of ${ }^{1} \mathrm{H}$ NMR spectroscopic data for natural and synthetic (+)-psigudial B (1).

\begin{tabular}{|c|c|c|}
\hline $\begin{array}{l}\text { carbon } \\
\text { number }\end{array}$ & $\begin{array}{l}\text { Natural (+)-psiguadial } \mathbf{B}^{11} \\
{ }^{1} \mathrm{H} \mathrm{NMR}, 500 \mathrm{MHz}, \mathrm{CDCl}_{3}\end{array}$ & $\begin{array}{l}\text { Synthetic (+)-psiguadial B } \\
{ }^{1} \mathrm{H} \mathrm{NMR}, 400 \mathrm{MHz}, \mathrm{CDCl}_{3}\end{array}$ \\
\hline 5'-OH & $\delta 13.51(\mathrm{~s}, 1 \mathrm{H})$ & $\delta 13.51(\mathrm{~s}, 1 \mathrm{H})$ \\
\hline 7'-OH & $13.04(\mathrm{~s}, 1 \mathrm{H})$ & $13.04(\mathrm{~s}, 1 \mathrm{H})$ \\
\hline $14^{\prime}, 15^{\prime}$ & $10.08(\mathrm{~s}, 2 \mathrm{H})$ & $10.07(\mathrm{~s}, 2 \mathrm{H})$ \\
\hline $9^{\prime}, 13^{\prime 12}$ & $7.23(2 \mathrm{H})$ & $7.26(\mathrm{dd}, J=14.6,1.5 \mathrm{~Hz}, 2 \mathrm{H})$ \\
\hline $11^{\prime}$ & $7.18(3 \mathrm{H})$ & $7.23-7.17(\mathrm{~m}, 1 \mathrm{H})$ \\
\hline $10^{\prime}, 12^{\prime}$ & - & $7.10($ br m, $2 \mathrm{H})$ \\
\hline $1^{\prime}$ & $3.49(\mathrm{~d}, J=11.5 \mathrm{~Hz}, 1 \mathrm{H})$ & $3.49(\mathrm{~d}, J=11.5 \mathrm{~Hz}, 1 \mathrm{H})$ \\
\hline 2 & $2.16(1 \mathrm{H})$ & $2.20-2.12(\mathrm{~m}, 1 \mathrm{H})$ \\
\hline 12 & $2.08(1 \mathrm{H})$ & $2.09(\mathrm{dd}, J=12.7,2.4 \mathrm{~Hz}, 1 \mathrm{H})$ \\
\hline 7 & $1.93(1 \mathrm{H})$ & $1.92(\mathrm{ddd}, J=14.9,12.8,4.2 \mathrm{~Hz}, 1 \mathrm{H})$ \\
\hline 5 & $1.82(\mathrm{~m}, 1 \mathrm{H})$ & $1.82(\mathrm{ddd}, J=12.3,8.8,5.6 \mathrm{~Hz}, 1 \mathrm{H})$ \\
\hline 9 & $1.68(1 \mathrm{H})$ & $1.73-1.59(\mathrm{~m}, 3 \mathrm{H})$ \\
\hline 6 & $1.65(1 \mathrm{H})$ & - \\
\hline 7 & $1.58(\mathrm{~m}, 1 \mathrm{H})$ & - \\
\hline 3 & $1.52(1 \mathrm{H})$ & $1.53-1.44(\mathrm{~m}, 1 \mathrm{H})$ \\
\hline 10 & $1.49(\mathrm{~m}, 2 \mathrm{H})$ & $1.49(\mathrm{ddd}, J=11.6,8.1,2.9 \mathrm{~Hz}, 2 \mathrm{H})$ \\
\hline 6,11 & $1.41(2 \mathrm{H})$ & - \\
\hline 3 & $1.37(1 \mathrm{H})$ & $1.44-1.29(\mathrm{~m}, 4 \mathrm{H})$ \\
\hline 12 & $1.29(1 \mathrm{H})$ & - \\
\hline 11 & $1.10(1 \mathrm{H})$ & $1.05(\mathrm{dd}, J=7.6,5.8 \mathrm{~Hz}, 1 \mathrm{H})$ \\
\hline 13 & $1.02(\mathrm{~s}, 3 \mathrm{H})$ & $1.02(\mathrm{~s}, 3 \mathrm{H})$ \\
\hline 14 & $1.01(\mathrm{~s}, 3 \mathrm{H})$ & $1.00(\mathrm{~s}, 3 \mathrm{H})$ \\
\hline 15 & $0.86(\mathrm{~s}, 3 \mathrm{H})$ & $0.85(\mathrm{~s}, 3 \mathrm{H})$ \\
\hline
\end{tabular}

(+)-psiguadial B (1) carbon numbering as reported by Shao et al. ${ }^{11}$

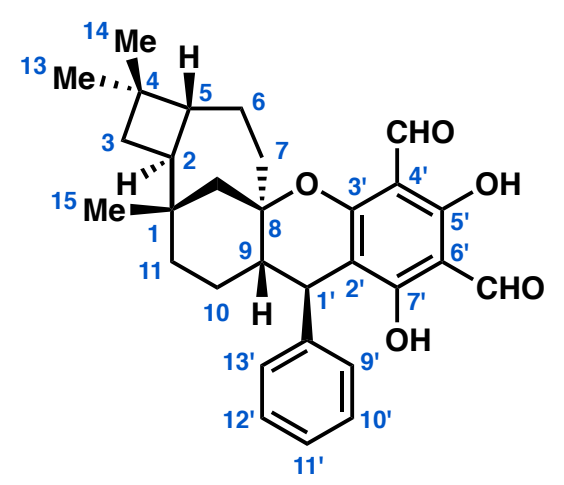


Comparison of ${ }^{13} \mathrm{C}$ NMR spectroscopic data for natural and synthetic (+)-psigudial B (1).

\begin{tabular}{|c|c|c|c|}
\hline $\begin{array}{l}\text { carbon } \\
\text { number }\end{array}$ & $\begin{array}{l}\text { Natural (+)-psiguadial B } \mathbf{B}^{11} \\
{ }^{13} \mathrm{C} \text { NMR, } 125 \mathrm{MHz}, \mathrm{CDCl}_{3}\end{array}$ & $\begin{array}{l}\text { Synthetic (+)-psiguadial } \mathbf{B}^{13} \\
{ }^{13} \mathrm{C} \mathrm{NMR}, 101 \mathrm{MHz}, \mathrm{CDCl}_{3}\end{array}$ & $\Delta$ \\
\hline $15^{\prime}$ & 192.3 & 192.3 & 0.0 \\
\hline $14^{\prime}$ & 191.4 & 191.5 & 0.1 \\
\hline $7^{\prime}$ & 169.6 & 169.6 & 0.0 \\
\hline $5^{\prime}$ & 168.5 & 168.5 & 0.0 \\
\hline 31 & 163.5 & 163.5 & 0.0 \\
\hline $8^{\prime}$ & 143.4 & 143.4 & 0.0 \\
\hline $9^{\prime}, 11^{\prime}, 13^{\prime}$ & 128.2 & 128.2 & 0.0 \\
\hline $10^{\prime}, 12^{\prime}$ & 126.2 & 126.2 & 0.0 \\
\hline $2^{\prime}$ & 105.7 & 105.7 & 0.0 \\
\hline $4^{\prime}$ & 104.6 & 104.6 & 0.0 \\
\hline $6^{\prime}$ & 104.2 & 104.1 & -0.1 \\
\hline 8 & 84.1 & 84.1 & 0.0 \\
\hline 9 & 50.0 & 50.0 & 0.0 \\
\hline 12 & 47.5 & 47.4 & -0.1 \\
\hline 5 & 44.1 & 44.0 & -0.1 \\
\hline $1^{\prime}$ & 40.4 & 40.4 & 0.0 \\
\hline 11 & 37.6 & 37.6 & 0.0 \\
\hline 2 & 37.0 & 36.9 & -0.1 \\
\hline 3 & 35.5 & 35.4 & -0.1 \\
\hline 4 & 35.1 & 35.1 & 0.0 \\
\hline 1 & 33.4 & 33.4 & 0.0 \\
\hline 13 & 30.6 & 30.6 & 0.0 \\
\hline 7 & 29.4 & 29.3 & -0.1 \\
\hline 15 & 26.1 & 26.1 & 0.0 \\
\hline 10 & 23.9 & 23.9 & 0.0 \\
\hline 14 & 20.7 & 20.7 & 0.0 \\
\hline 6 & 20.1 & 20.1 & 0.0 \\
\hline
\end{tabular}


${ }^{1}$ H NMR spectral comparison of natural and synthetic (+)-psigudial B (1).

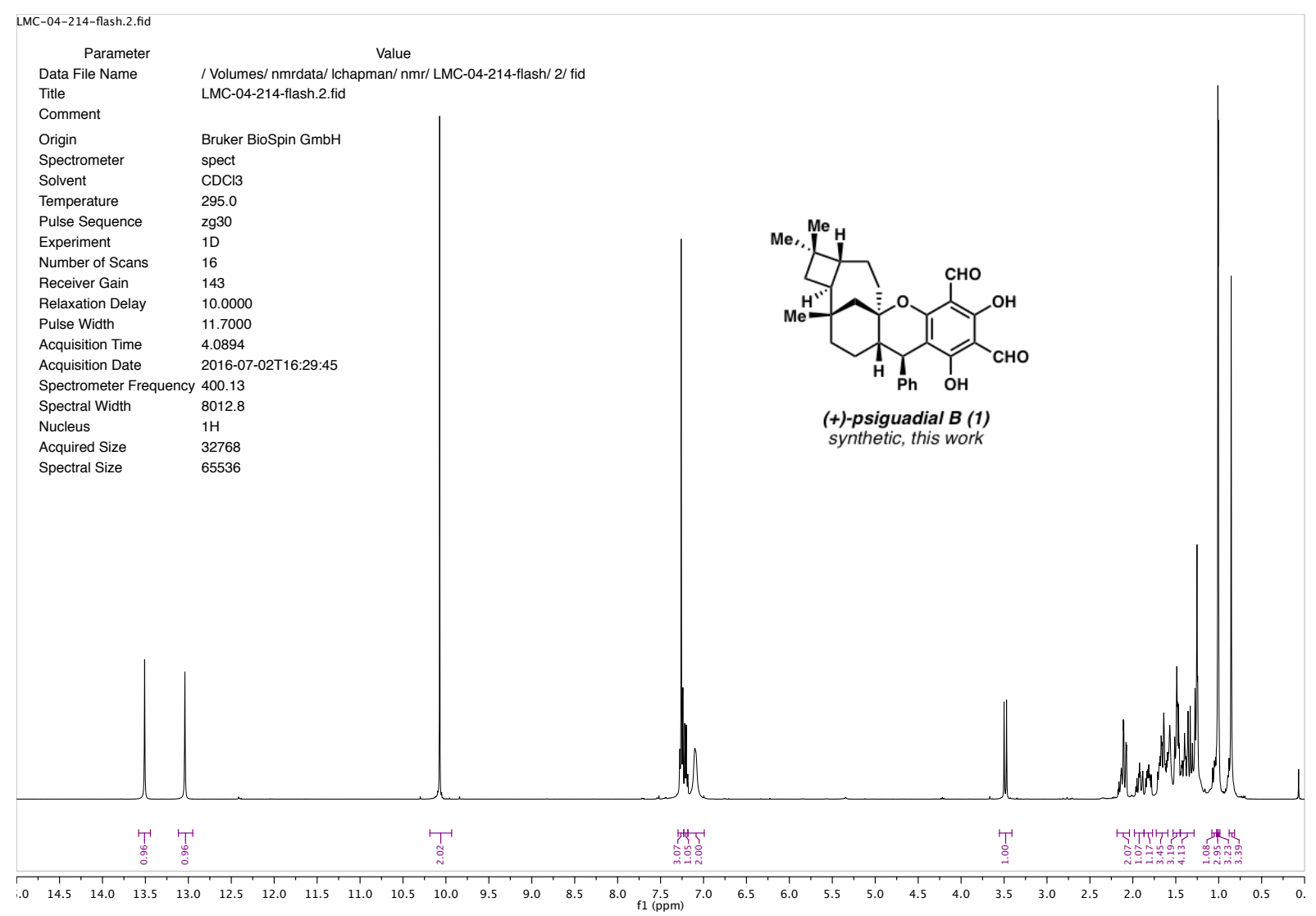

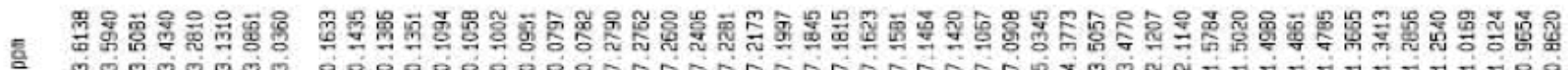
送 IIIT

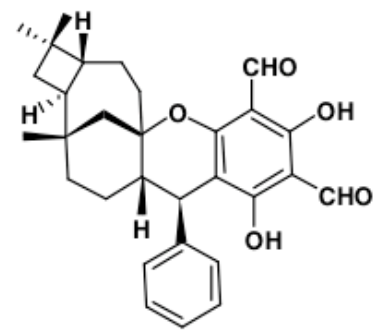

Natural sample, reported by Shao et al. Org. Lett. 2010, 12, 5040.

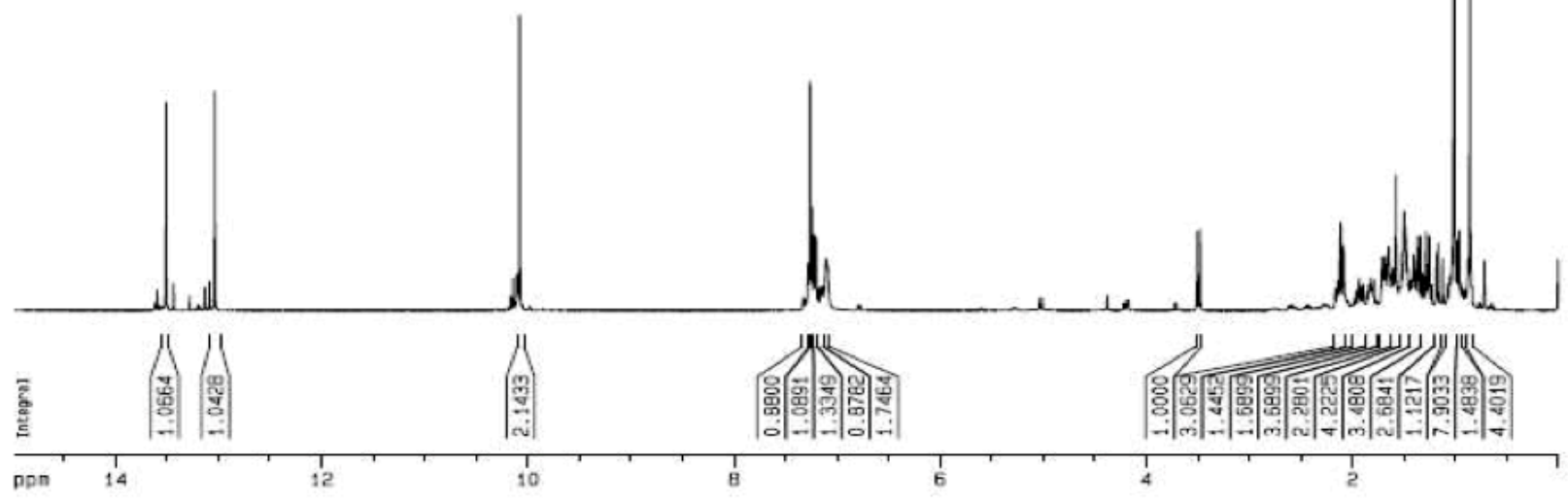


${ }^{13}$ C NMR spectral comparison of natural and synthetic (+)-psigudial B (1).
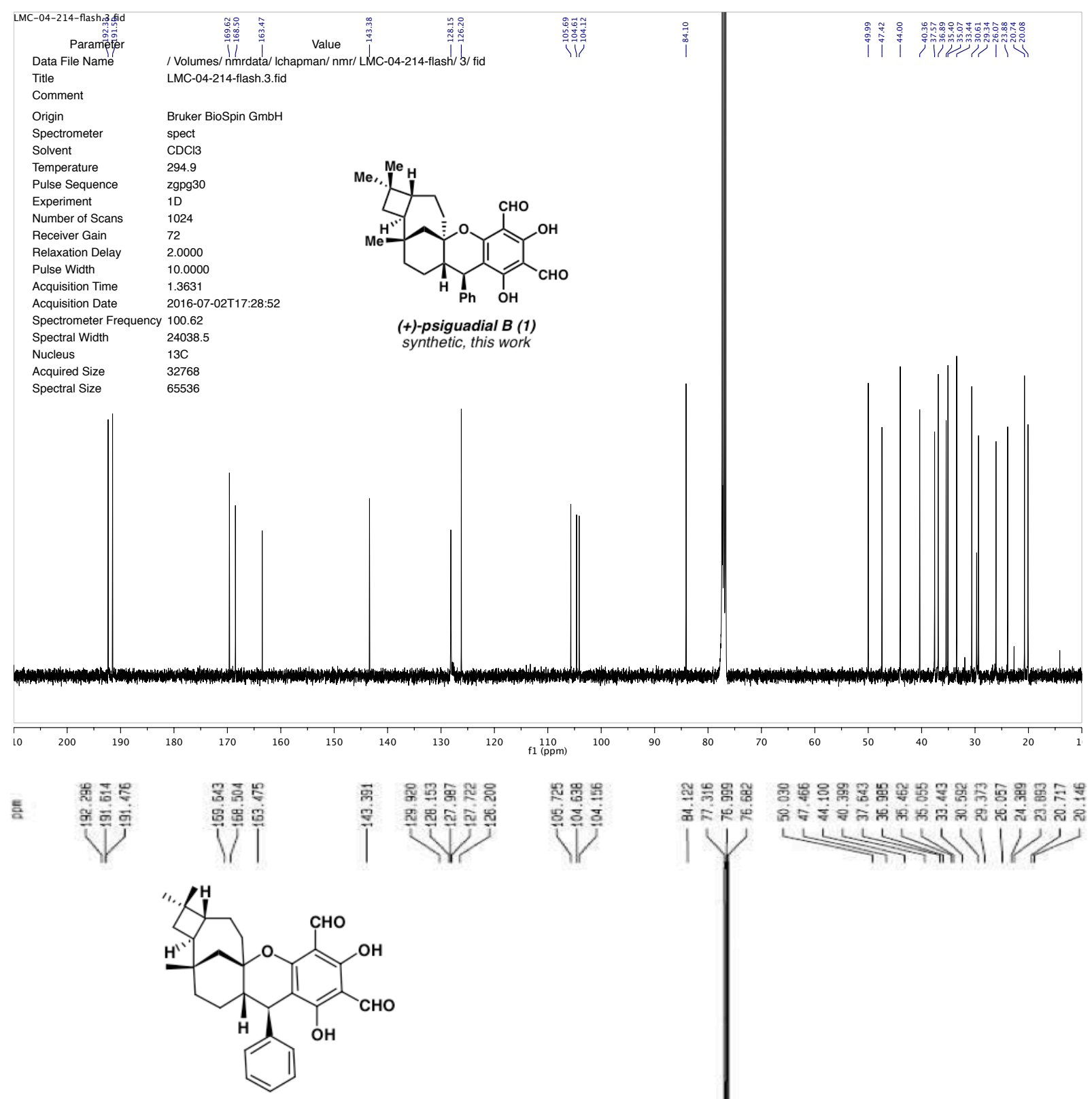

Natural sample, reported by Shao et al. Org. Lett. 2010, 12, 5040.

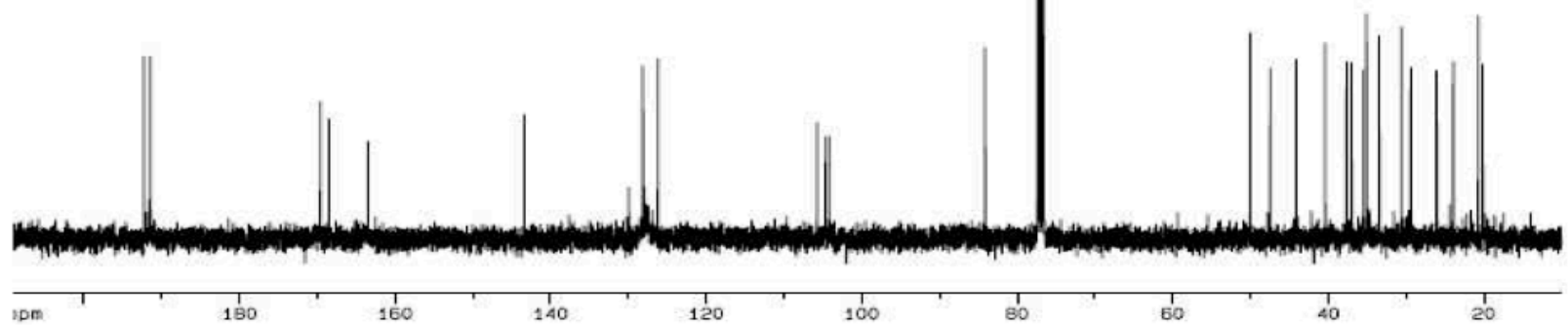




\section{X-Ray crystallographic data for 11.}

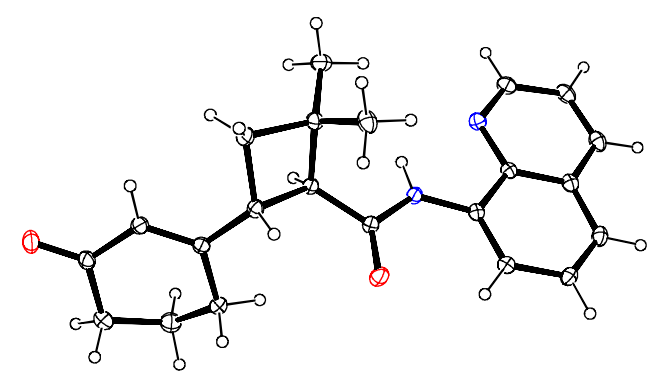

Table 1. Crystal data and structure refinement for crystal01.

Identification code

Empirical formula

Formula weight

Temperature

Wavelength

Crystal system

Space group

Unit cell dimensions

Volume

Z

Density (calculated)

Absorption coefficient

$\mathrm{F}(000)$

Crystal size

Theta range for data collection

Index ranges

Reflections collected

Independent reflections

Completeness to theta $=67.679^{\circ}$

Absorption correction

Max. and min. transmission

Refinement method

Data / restraints / parameters

Goodness-of-fit on $\mathrm{F}^{2}$

Final $\mathrm{R}$ indices [I>2sigma(I)]
Crystal01

$\mathrm{C}_{22} \mathrm{H}_{24} \mathrm{~N}_{2} \mathrm{O}_{2}$

348.43

$119.98 \mathrm{~K}$

$1.54178 \AA$

Orthorhombic

$\mathrm{P} 22_{1} 2_{1}$

$\mathrm{a}=6.3942(5) \AA$

$\alpha=90^{\circ}$.

$\mathrm{b}=11.6678(9) \AA$

$\beta=90^{\circ}$.

$\mathrm{c}=24.848(2) \AA$

$\gamma=90^{\circ}$.
4

$1.248 \mathrm{Mg} / \mathrm{m}^{3}$

$0.636 \mathrm{~mm}^{-1}$

744

$0.237 \times 0.093 \times 0.082 \mathrm{~mm}^{3}$

3.557 to $71.792^{\circ}$.

$-7<=\mathrm{h}<=7,-14<=\mathrm{k}<=14,-28<=\mathrm{l}<=30$

41642

$3620[\mathrm{R}(\mathrm{int})=0.0313]$

$99.9 \%$

Semi-empirical from equivalents

0.9883 and 0.8875

Full-matrix least-squares on $\mathrm{F}^{2}$

$3620 / 0 / 237$

1.043

$\mathrm{R} 1=0.0292, \mathrm{wR} 2=0.0782$ 
$\mathrm{R}$ indices (all data)

Absolute structure parameter

Largest diff. peak and hole
$\mathrm{R} 1=0.0294, \mathrm{wR} 2=0.0785$

$-0.04(4)$

0.255 and $-0.181 \mathrm{e} / \AA^{-3}$

Table 2. Atomic coordinates $\left(\mathrm{x} 10^{4}\right)$ and equivalent isotropic displacement parameters $\left(\AA^{2} \times 10^{3}\right)$ for crysta101. $U(\mathrm{eq})$ is defined as one third of the trace of the orthogonalized $\mathrm{U}^{\mathrm{ij}}$ tensor.

\begin{tabular}{|c|c|c|c|c|}
\hline & $\mathrm{x}$ & $\mathrm{y}$ & $\mathrm{z}$ & $\mathrm{U}(\mathrm{eq})$ \\
\hline $\mathrm{O}(1)$ & $-4024(2)$ & $4325(1)$ & $3741(1)$ & $30(1)$ \\
\hline $\mathrm{O}(2)$ & 5981(2) & $5004(1)$ & 2361(1) & $34(1)$ \\
\hline $\mathrm{N}(1)$ & $-3297(2)$ & $4719(1)$ & $4622(1)$ & $22(1)$ \\
\hline $\mathrm{N}(2)$ & $-3330(2)$ & $4949(1)$ & $5683(1)$ & $23(1)$ \\
\hline $\mathrm{C}(1)$ & $-2893(2)$ & $4755(1)$ & 4081(1) & $21(1)$ \\
\hline$C(2)$ & $-944(2)$ & $5426(1)$ & $3950(1)$ & 19(1) \\
\hline$C(3)$ & $-1002(3)$ & $6755(1)$ & $4055(1)$ & $24(1)$ \\
\hline $\mathrm{C}(4)$ & $-3194(3)$ & $7259(2)$ & 3999(1) & $31(1)$ \\
\hline$C(5)$ & $36(3)$ & $7135(2)$ & $4574(1)$ & $33(1)$ \\
\hline $\mathrm{C}(6)$ & $286(3)$ & 6893(1) & $3529(1)$ & $26(1)$ \\
\hline$C(7)$ & $-388(3)$ & $5674(1)$ & $3354(1)$ & $22(1)$ \\
\hline $\mathrm{C}(8)$ & 1149(3) & 4904(1) & $3080(1)$ & $22(1)$ \\
\hline $\mathrm{C}(9)$ & 2914(3) & $5310(1)$ & $2858(1)$ & $23(1)$ \\
\hline$C(10)$ & 4426(3) & $4596(2)$ & $2574(1)$ & $26(1)$ \\
\hline$C(11)$ & 3943(3) & $3338(2)$ & $2530(1)$ & $38(1)$ \\
\hline $\mathrm{C}(12)$ & 2522(3) & 2898(2) & 2970(1) & $40(1)$ \\
\hline$C(13)$ & $612(3)$ & $3648(2)$ & $3047(1)$ & $30(1)$ \\
\hline$C(14)$ & $-5080(2)$ & $4317(1)$ & $4886(1)$ & $20(1)$ \\
\hline$C(15)$ & $-6783(3)$ & $3820(1)$ & $4640(1)$ & $22(1)$ \\
\hline$C(16)$ & $-8492(3)$ & $3441(1)$ & $4955(1)$ & $24(1)$ \\
\hline$C(17)$ & $-8509(3)$ & $3560(1)$ & $5503(1)$ & $27(1)$ \\
\hline$C(18)$ & $-6795(3)$ & $4077(1)$ & $5768(1)$ & $24(1)$ \\
\hline $\mathrm{C}(19)$ & $-5062(2)$ & $4456(1)$ & $5460(1)$ & $20(1)$ \\
\hline$C(20)$ & $-3303(3)$ & $5073(2)$ & $6212(1)$ & $27(1)$ \\
\hline $\mathrm{C}(21)$ & $-4952(3)$ & $4734(2)$ & $6553(1)$ & $29(1)$ \\
\hline $\mathrm{C}(22)$ & $-6681(3)$ & $4240(2)$ & $6333(1)$ & $29(1)$ \\
\hline
\end{tabular}


Table 3. Bond lengths $[\AA]$ and angles $\left[{ }^{\circ}\right]$ for crystal01.

\begin{tabular}{|c|c|}
\hline $\mathrm{O}(1)-\mathrm{C}(1)$ & $1.219(2)$ \\
\hline $\mathrm{O}(2)-\mathrm{C}(10)$ & $1.224(2)$ \\
\hline $\mathrm{N}(1)-\mathrm{C}(1)$ & $1.369(2)$ \\
\hline $\mathrm{N}(1)-\mathrm{C}(14)$ & $1.397(2)$ \\
\hline $\mathrm{N}(2)-\mathrm{C}(19)$ & $1.365(2)$ \\
\hline $\mathrm{N}(2)-\mathrm{C}(20)$ & $1.320(2)$ \\
\hline $\mathrm{C}(1)-\mathrm{C}(2)$ & $1.507(2)$ \\
\hline$C(2)-C(3)$ & $1.574(2)$ \\
\hline$C(3)-C(4)$ & $1.526(2)$ \\
\hline$C(3)-C(6)$ & $1.554(2)$ \\
\hline$C(5)-C(3)$ & $1.516(2)$ \\
\hline$C(7)-C(2)$ & $1.551(2)$ \\
\hline$C(7)-C(6)$ & $1.549(2)$ \\
\hline$C(7)-C(8)$ & $1.495(2)$ \\
\hline $\mathrm{C}(8)-\mathrm{C}(13)$ & $1.508(2)$ \\
\hline$C(8)-C(9)$ & $1.342(2)$ \\
\hline $\mathrm{C}(10)-\mathrm{C}(11)$ & $1.504(3)$ \\
\hline$C(10)-C(9)$ & $1.459(2)$ \\
\hline$C(11)-C(12)$ & $1.511(3)$ \\
\hline$C(13)-C(12)$ & $1.515(3)$ \\
\hline$C(14)-C(15)$ & $1.377(2)$ \\
\hline$C(14)-C(19)$ & $1.436(2)$ \\
\hline$C(15)-C(16)$ & $1.414(2)$ \\
\hline$C(17)-C(16)$ & $1.369(2)$ \\
\hline $\mathrm{C}(18)-\mathrm{C}(17)$ & $1.415(2)$ \\
\hline$C(18)-C(19)$ & $1.417(2)$ \\
\hline $\mathrm{C}(18)-\mathrm{C}(22)$ & $1.417(2)$ \\
\hline$C(20)-C(21)$ & $1.410(3)$ \\
\hline$C(21)-C(22)$ & $1.362(3)$ \\
\hline $\mathrm{O}(1)-\mathrm{C}(1)-\mathrm{C}(2)$ & $123.68(14)$ \\
\hline $\mathrm{O}(1)-\mathrm{C}(1)-\mathrm{N}(1)$ & $123.69(14)$ \\
\hline $\mathrm{O}(2)-\mathrm{C}(10)-\mathrm{C}(11)$ & $121.04(16)$ \\
\hline $\mathrm{O}(2)-\mathrm{C}(10)-\mathrm{C}(9)$ & $121.70(16)$ \\
\hline $\mathrm{N}(1)-\mathrm{C}(1)-\mathrm{C}(2)$ & $112.58(13)$ \\
\hline
\end{tabular}




\begin{tabular}{|c|c|}
\hline $\mathrm{N}(1)-\mathrm{C}(14)-\mathrm{C}(19)$ & $115.04(14)$ \\
\hline $\mathrm{N}(2)-\mathrm{C}(19)-\mathrm{C}(14)$ & $117.20(14)$ \\
\hline $\mathrm{N}(2)-\mathrm{C}(19)-\mathrm{C}(18)$ & $123.12(14)$ \\
\hline $\mathrm{N}(2)-\mathrm{C}(20)-\mathrm{C}(21)$ & $123.85(16)$ \\
\hline$C(1)-C(2)-C(3)$ & $117.15(13)$ \\
\hline$C(1)-C(2)-C(7)$ & $119.57(13)$ \\
\hline $\mathrm{C}(1)-\mathrm{N}(1)-\mathrm{C}(14)$ & $128.75(13)$ \\
\hline $\mathrm{C}(4)-\mathrm{C}(3)-\mathrm{C}(2)$ & $112.66(14)$ \\
\hline $\mathrm{C}(4)-\mathrm{C}(3)-\mathrm{C}(6)$ & $111.66(14)$ \\
\hline$C(5)-C(3)-C(2)$ & $114.81(13)$ \\
\hline$C(5)-C(3)-C(4)$ & $111.60(14)$ \\
\hline$C(5)-C(3)-C(6)$ & $116.97(15)$ \\
\hline $\mathrm{C}(6)-\mathrm{C}(3)-\mathrm{C}(2)$ & $87.09(11)$ \\
\hline$C(6)-C(7)-C(2)$ & $88.10(12)$ \\
\hline$C(7)-C(2)-C(3)$ & $88.86(11)$ \\
\hline$C(7)-C(6)-C(3)$ & $89.69(12)$ \\
\hline$C(7)-C(8)-C(13)$ & $117.31(14)$ \\
\hline $\mathrm{C}(8)-\mathrm{C}(13)-\mathrm{C}(12)$ & $112.61(15)$ \\
\hline$C(8)-C(7)-C(2)$ & $118.23(13)$ \\
\hline$C(8)-C(7)-C(6)$ & $119.83(14)$ \\
\hline$C(8)-C(9)-C(10)$ & $123.62(15)$ \\
\hline$C(9)-C(10)-C(11)$ & $117.19(15)$ \\
\hline$C(9)-C(8)-C(13)$ & $120.87(15)$ \\
\hline $\mathrm{C}(9)-\mathrm{C}(8)-\mathrm{C}(7)$ & $121.80(15)$ \\
\hline$C(10)-C(11)-C(12)$ & $113.71(15)$ \\
\hline$C(11)-C(12)-C(13)$ & $112.36(17)$ \\
\hline$C(14)-C(15)-C(16)$ & $119.81(14)$ \\
\hline$C(15)-C(14)-C(19)$ & $119.65(14)$ \\
\hline $\mathrm{C}(15)-\mathrm{C}(14)-\mathrm{N}(1)$ & $125.31(14)$ \\
\hline$C(16)-C(17)-C(18)$ & $120.05(16)$ \\
\hline$C(17)-C(16)-C(15)$ & $121.66(16)$ \\
\hline$C(17)-C(18)-C(19)$ & $119.14(14)$ \\
\hline$C(17)-C(18)-C(22)$ & $123.96(16)$ \\
\hline$C(18)-C(19)-C(14)$ & $119.67(14)$ \\
\hline $\mathrm{C}(20)-\mathrm{N}(2)-\mathrm{C}(19)$ & $117.41(15)$ \\
\hline$C(21)-C(22)-C(18)$ & $119.72(16)$ \\
\hline $\mathrm{C}(22)-\mathrm{C}(18)-\mathrm{C}(19)$ & $116.89(15)$ \\
\hline
\end{tabular}


Table 4. Anisotropic displacement parameters $\left(\AA^{2} \times 10^{3}\right)$ for crystal01. The anisotropic displacement factor exponent takes the form: $-2 \pi^{2}\left[h^{2} a^{* 2} U^{11}+\ldots+2 h k a^{*} b^{*} U^{12}\right]$

\begin{tabular}{lllllll}
\hline & $\mathrm{U}^{11}$ & $\mathrm{U}^{22}$ & $\mathrm{U}^{33}$ & $\mathrm{U}^{23}$ & $\mathrm{U}^{13}$ & $\mathrm{U}^{12}$ \\
\hline $\mathrm{O}(1)$ & $28(1)$ & $43(1)$ & $19(1)$ & $1(1)$ & $-2(1)$ & $-12(1)$ \\
$\mathrm{O}(2)$ & $28(1)$ & $46(1)$ & $27(1)$ & $0(1)$ & $8(1)$ & $-4(1)$ \\
$\mathrm{N}(1)$ & $20(1)$ & $28(1)$ & $18(1)$ & $0(1)$ & $0(1)$ & $-4(1)$ \\
$\mathrm{N}(2)$ & $24(1)$ & $25(1)$ & $21(1)$ & $1(1)$ & $-1(1)$ & $1(1)$ \\
$\mathrm{C}(1)$ & $20(1)$ & $22(1)$ & $20(1)$ & $2(1)$ & $-1(1)$ & $0(1)$ \\
$\mathrm{C}(2)$ & $19(1)$ & $22(1)$ & $17(1)$ & $1(1)$ & $-1(1)$ & $-1(1)$ \\
$\mathrm{C}(3)$ & $26(1)$ & $23(1)$ & $23(1)$ & $0(1)$ & $4(1)$ & $-2(1)$ \\
$\mathrm{C}(4)$ & $34(1)$ & $28(1)$ & $32(1)$ & $4(1)$ & $7(1)$ & $8(1)$ \\
$\mathrm{C}(5)$ & $38(1)$ & $31(1)$ & $29(1)$ & $-6(1)$ & $2(1)$ & $-8(1)$ \\
$\mathrm{C}(6)$ & $29(1)$ & $23(1)$ & $27(1)$ & $2(1)$ & $5(1)$ & $-2(1)$ \\
$\mathrm{C}(7)$ & $22(1)$ & $26(1)$ & $18(1)$ & $4(1)$ & $0(1)$ & $-2(1)$ \\
$\mathrm{C}(8)$ & $22(1)$ & $26(1)$ & $16(1)$ & $-1(1)$ & $-2(1)$ & $-2(1)$ \\
$\mathrm{C}(9)$ & $26(1)$ & $25(1)$ & $19(1)$ & $1(1)$ & $0(1)$ & $-4(1)$ \\
$\mathrm{C}(10)$ & $24(1)$ & $37(1)$ & $16(1)$ & $-1(1)$ & $0(1)$ & $-3(1)$ \\
$\mathrm{C}(11)$ & $36(1)$ & $39(1)$ & $40(1)$ & $-16(1)$ & $10(1)$ & $-3(1)$ \\
$\mathrm{C}(12)$ & $45(1)$ & $27(1)$ & $48(1)$ & $-8(1)$ & $12(1)$ & $-4(1)$ \\
$\mathrm{C}(13)$ & $31(1)$ & $28(1)$ & $32(1)$ & $-6(1)$ & $8(1)$ & $-8(1)$ \\
$\mathrm{C}(14)$ & $21(1)$ & $18(1)$ & $21(1)$ & $3(1)$ & $1(1)$ & $3(1)$ \\
$\mathrm{C}(15)$ & $24(1)$ & $20(1)$ & $21(1)$ & $2(1)$ & $-1(1)$ & $2(1)$ \\
$\mathrm{C}(16)$ & $21(1)$ & $22(1)$ & $31(1)$ & $3(1)$ & $0(1)$ & $-1(1)$ \\
$\mathrm{C}(17)$ & $24(1)$ & $26(1)$ & $31(1)$ & $5(1)$ & $7(1)$ & $-1(1)$ \\
$\mathrm{C}(18)$ & $26(1)$ & $20(1)$ & $24(1)$ & $3(1)$ & $3(1)$ & $4(1)$ \\
$\mathrm{C}(19)$ & $22(1)$ & $17(1)$ & $21(1)$ & $2(1)$ & $0(1)$ & $3(1)$ \\
$\mathrm{C}(20)$ & $32(1)$ & $28(1)$ & $22(1)$ & $-1(1)$ & $-3(1)$ & $2(1)$ \\
$\mathrm{C}(21)$ & $38(1)$ & $30(1)$ & $20(1)$ & $1(1)$ & $2(1)$ & $6(1)$ \\
$\mathrm{C}(22)$ & $34(1)$ & $28(1)$ & $25(1)$ & $4(1)$ & $8(1)$ & $4(1)$ \\
& & & & & & \\
\hline
\end{tabular}


Table 5. Hydrogen coordinates ( x $\left.10^{4}\right)$ and isotropic displacement parameters $\left(\AA^{2} \times 10^{3}\right)$ for crystal01.

\begin{tabular}{|c|c|c|c|c|}
\hline & $\mathrm{x}$ & $\mathrm{y}$ & $\mathrm{z}$ & $\mathrm{U}(\mathrm{eq})$ \\
\hline $\mathrm{H}(1)$ & -2298 & 4985 & 4831 & 26 \\
\hline $\mathrm{H}(2)$ & 286 & 5078 & 4137 & 23 \\
\hline $\mathrm{H}(4 \mathrm{~A})$ & -3840 & 6972 & 3667 & 47 \\
\hline $\mathrm{H}(4 \mathrm{~B})$ & -3104 & 8096 & 3983 & 47 \\
\hline $\mathrm{H}(4 \mathrm{C})$ & -4046 & 7031 & 4309 & 47 \\
\hline $\mathrm{H}(5 \mathrm{~A})$ & -803 & 6878 & 4881 & 49 \\
\hline $\mathrm{H}(5 \mathrm{~B})$ & 144 & 7973 & 4579 & 49 \\
\hline $\mathrm{H}(5 \mathrm{C})$ & 1438 & 6799 & 4597 & 49 \\
\hline $\mathrm{H}(6 \mathrm{~A})$ & -237 & 7508 & 3290 & 31 \\
\hline $\mathrm{H}(6 \mathrm{~B})$ & 1809 & 6972 & 3590 & 31 \\
\hline $\mathrm{H}(7)$ & -1698 & 5721 & 3135 & 26 \\
\hline $\mathrm{H}(9)$ & 3195 & 6107 & 2889 & 28 \\
\hline $\mathrm{H}(11 \mathrm{~A})$ & 5272 & 2902 & 2541 & 46 \\
\hline $\mathrm{H}(11 \mathrm{~B})$ & 3277 & 3190 & 2177 & 46 \\
\hline $\mathrm{H}(12 \mathrm{~A})$ & 3314 & 2863 & 3312 & 48 \\
\hline $\mathrm{H}(12 \mathrm{~B})$ & 2067 & 2110 & 2880 & 48 \\
\hline $\mathrm{H}(13 \mathrm{~A})$ & -363 & 3523 & 2743 & 36 \\
\hline $\mathrm{H}(13 \mathrm{~B})$ & -114 & 3417 & 3382 & 36 \\
\hline $\mathrm{H}(15)$ & -6811 & 3732 & 4260 & 26 \\
\hline $\mathrm{H}(16)$ & -9656 & 3096 & 4781 & 29 \\
\hline $\mathrm{H}(17)$ & -9674 & 3295 & 5705 & 32 \\
\hline $\mathrm{H}(20)$ & -2101 & 5411 & 6371 & 33 \\
\hline $\mathrm{H}(21)$ & -4858 & 4849 & 6930 & 35 \\
\hline $\mathrm{H}(22)$ & -7806 & 4005 & 6557 & 35 \\
\hline
\end{tabular}




\section{References}

1. Still, W. C., Kahn, M. \& Mitra, A. J. Org. Chem. 1978, 43, 2923.

2. Prepared according to: Ghosh, A.; Banerjee, U. K.; Venkateswaran, R. V. Tetrahedron 1990, 46 (8), 3077 , using $p$-4-acetamidobenzenesulfonyl azide ( $p$-ABSA) instead of tosyl azide. In our hands, the formylation of 2,2-dimethylcyclopentanone at the reported concentration $(1.5 \mathrm{M})$ reacts rapidly upon warming to room temperature (caution: extremely exothermic on large scale!). We found that more concentrated reaction mixtures $(2.2 \mathrm{M})$ react readily at $0{ }^{\circ} \mathrm{C}$, providing for a safer protocol.

3. Reaction time varies with the age of the lamp. A UV-opaque film slowly develops on the inside surface of the flask facing the lamp upon prolonged irradiation. This film can be removed by soaking the flask in an alkali base bath $\left(\mathrm{KOH}, 4: 1 i-\mathrm{PrOH} / \mathrm{H}_{2} \mathrm{O}\right)$.

4. Prepared according to: Piers, E.; Grierson, J. R.; Lau, C. K.; Nagakura, I. Can. J. Chem. 1982, 60, 210.

5. Dolomanov, O. V.; Bourhis, L. J.; Gildea, R. J.; Howard, J. A. K.; Puschmann, H. J. Appl. Cryst. 2009, 42, 339.

6. Sheldrick, G. M. Acta Cryst. A 2008, 64, 112.

7. See procedure on page S11.

8. Prepared according to the ligand protocol described in: Bao, H.; Qi, X.; Tambar, U. K. J. Am. Chem. Soc. 2011, 133 (5), 1206. We found that the use of DCM as a reaction solvent provided higher and more reproducible yields, compared with THF.

9. Prepared according to: Wong, N. C.W.; Tucker, J. E.L.; Hansen, H. C.; Chiacchia, F. S.; McCaffrey, D. Compounds for the Prevention and Treatment of Cardiovascular Diseases. U.S. patent 188467 A1, August, 7, 2008.

10. Extensive optimization aimed at increasing conversion of this reaction resulted in either significant decomposition, a variety of over-oxidation products, or trapping by 2,3-dichloro-5,6-dicyanohydroquinone. As a result, we found that the most efficient material throughput was achieved by running the reaction to $\sim 50 \%$ conversion using the above protocol, and resubjecting the recovered starting material to the reaction conditions

11. Isolation of psiguadials A and B: Shao, M.; Wang, Y.; Liu, Z.; Zhang, D.-M.; Cao, H.-H.; Jiang, R.-W.; Fan, C.-L.; Zhang, X.-Q.; Chen, H.-R.; Yao, X.-S.; Ye, W.-C. Org. Lett. 2010, 12, 5040. Except where designated, multiplicities are not specified.

12. Shao et al. report the two protons at $7.23 \mathrm{ppm}$ as corresponding to the carbon signal at $126.2 \mathrm{ppm}$ (carbons $10^{\prime}$ and 12'). This is assumed to be a typographical error based on the reported plot of their HSQC spectrum and our own data showing a correlation between the proton signal at $7.26 \mathrm{ppm}$ and the more downfield carbon shift at $128.2 \mathrm{ppm}$, which corresponds to carbons 9' and 13'. The remaining three aromatic proton signals are adjusted accordingly in the comparison list.

13. Center peak of $\mathrm{CDCl}_{3}$ is referenced to $76.999 \mathrm{ppm}$, as shown in the Shao et al. report, see ${ }^{13} \mathrm{C}$ spectral comparison on page $\mathrm{S} 31$. 


\title{
Enantioselective Total Synthesis of (+)-Psiguadial B
}

\author{
Lauren M. Chapman, Jordan C. Beck, Linglin Wu, and Sarah E. Reisman* \\ The Warren and Katharine Schlinger Laboratory of Chemistry and Chemical Engineering \\ Division of Chemistry and Chemical Engineering, California Institute of Technology \\ Pasadena, California 91125 \\ reisman@caltech.edu
}

Supporting Information 2 (Spectral Data) 


\section{JCB_cyclobutamide_060916.1.fid}

Parameter

Data File Name

Title

Origin

Solvent

Temperature

Pulse Sequence

Number of Scans

Receiver Gain

Relaxation Delay

Pulse Width

Acquisition Time

Acquisition Date

Spectrometer Freq

Spectral Width

Lowest Frequency

Nucleus

Acquired Size

Spectral Size

/ Volumes/nmrdata/jbeck/nmr/JCB_cyclobutamide_060916/ 1/ fid

JCB_cyclobutamide_060916.1.fid

Bruker BioSpin $\mathrm{GmbH}$

$\mathrm{CDCl} 3$

295.0

zg30

1

197

1.0000

11.7000

4.0894

2016-06-09T16:38:44

400.13

8012.8

$-1545.6$

$1 \mathrm{H}$

32768

65536
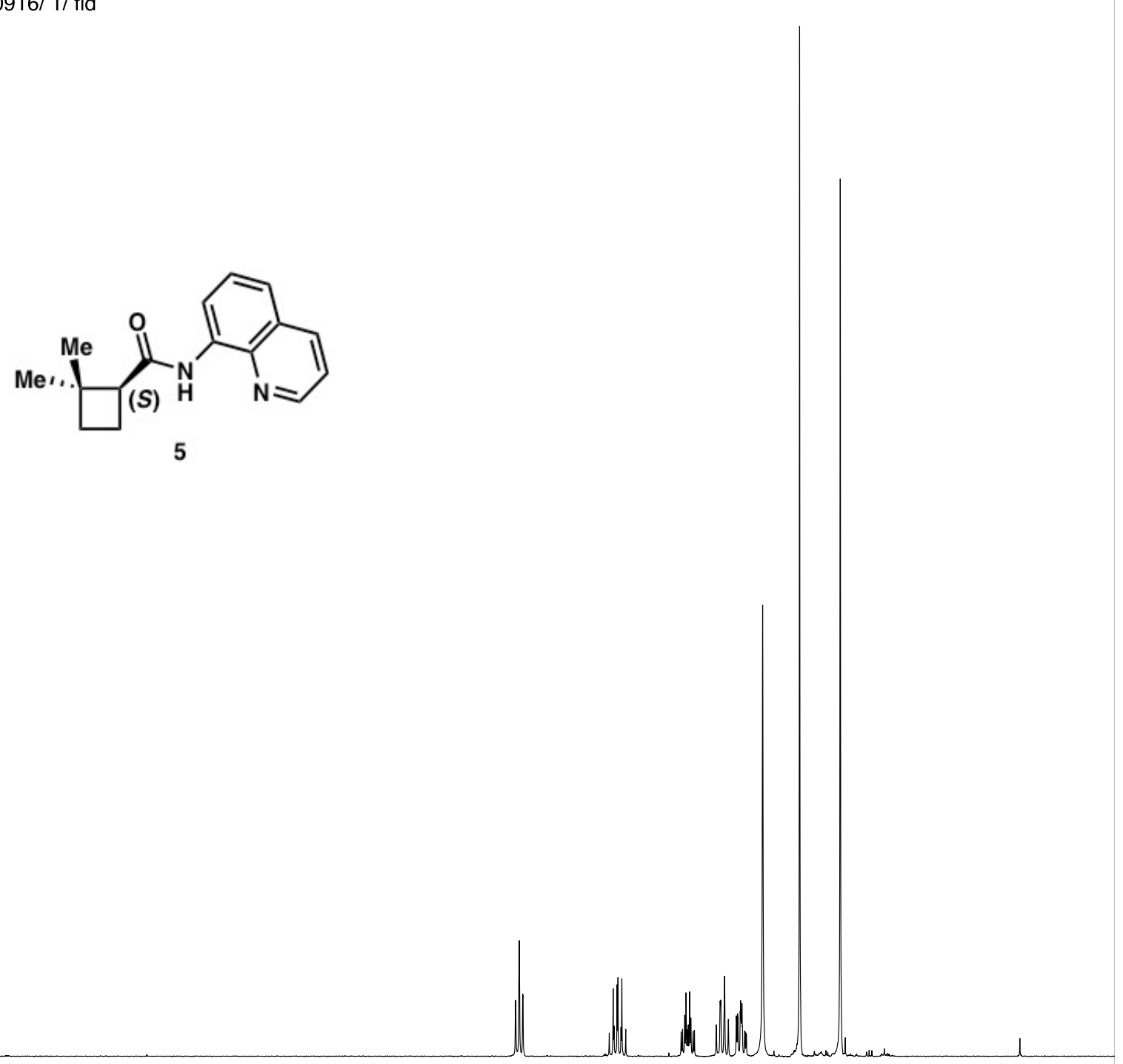

\begin{tabular}{|c|c|c|c|c|c|c|c|c|c|c|c|c|c|c|c|c|c|c|c|c|c|c|c|}
\hline & & & 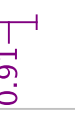 & $\begin{array}{l}\prod_{1} \\
\stackrel{0}{0} \\
\stackrel{i}{N}\end{array}$ & & $\begin{array}{l}T_{1} \\
-1 \\
0 \\
-1\end{array}$ & 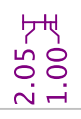 & & & & & & & & & 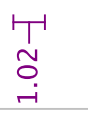 & 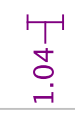 & 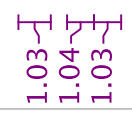 & $\begin{array}{l}1 \\
9 \\
\circ \\
\dot{m} \\
\dot{m}\end{array}$ & $\begin{array}{l}T \\
\stackrel{-1}{r} \\
\vec{m}\end{array}$ & & & \\
\hline 0 & 10.5 & 10.0 & 9.5 & 9.0 & 8.5 & 8.0 & 7.5 & 7.0 & 6.5 & 6.0 & $\begin{array}{l}5.5 \\
\mathrm{f} 1\end{array}$ & $\begin{array}{c}5.0 \\
\text { om) }\end{array}$ & 4.5 & 4.0 & 3.5 & 3.0 & 2.5 & 2.0 & 1.5 & 1.0 & 0.5 & 0.0 & -0 \\
\hline
\end{tabular}




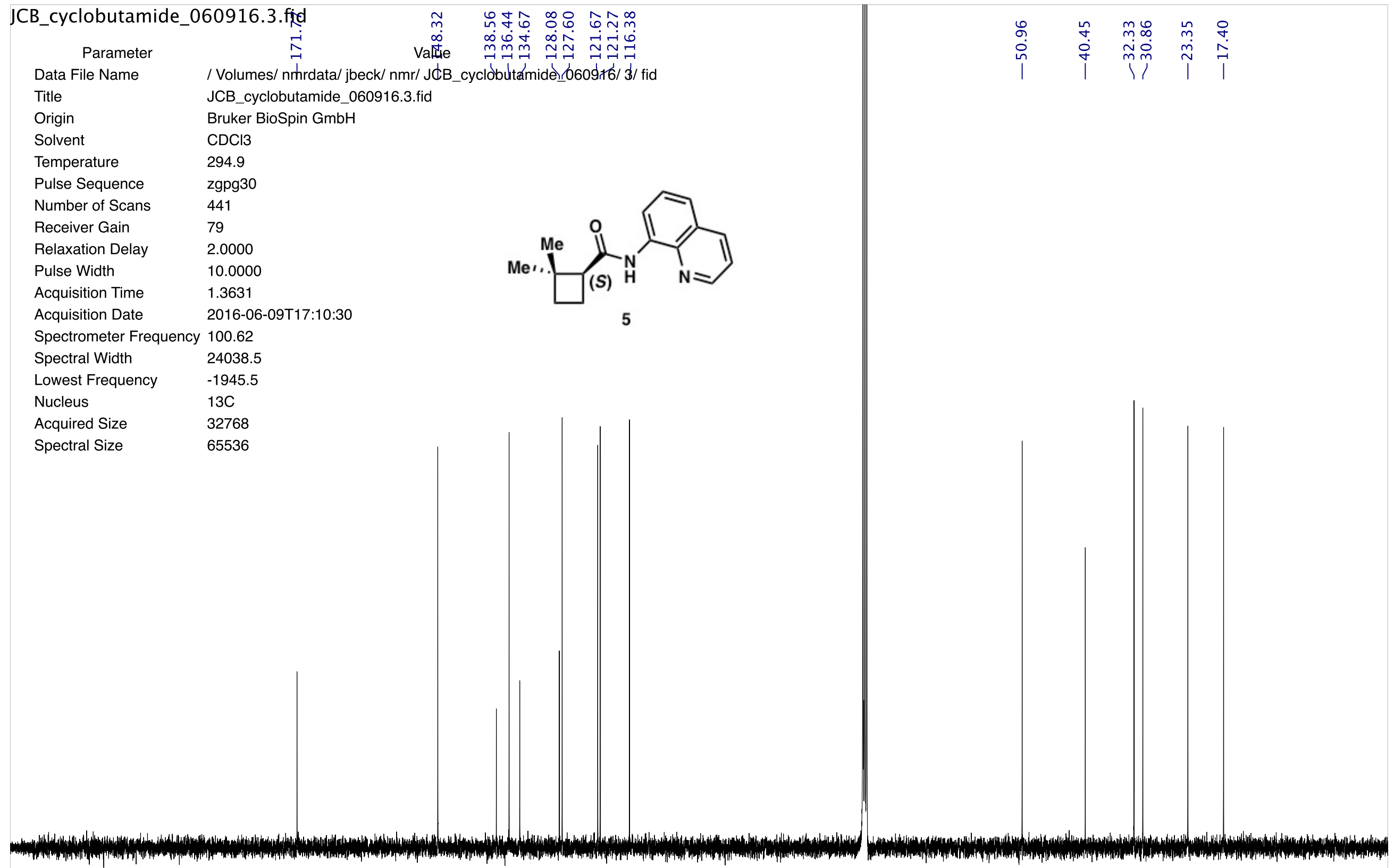

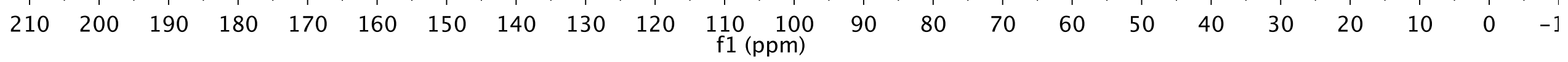




\section{PROTON01}

LMC-02-89

Data File Name / Volumes/ nmrdata-1/ Ichapman/ vnmrsys/ data/ LMC-02-89/ PROTON01.fid/ fid

Title PROTON01

Comment

LMC-02-89

Origin

Varian

Spectrometer inov

Solvent cdcl3

Temperature 25.0

Pulse Sequence s2pul

Experiment 1D

Probe autox7991

Number of Scans 8

Receiver Gain 34

Relaxation Delay 1.0000

Pulse Width $\quad 0.0000$

Acquisition Time 3.0000

Acquisition Date 2014-01-15T18:54:01

Nucleus

$1 \mathrm{H}$

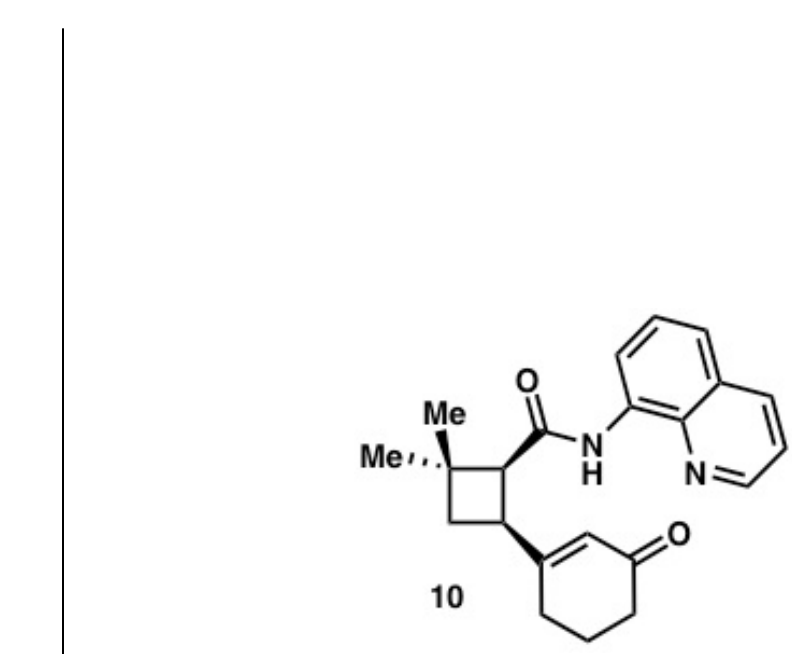

$\mid$

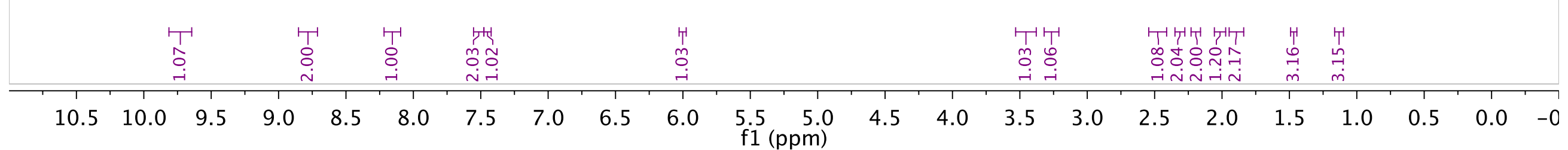




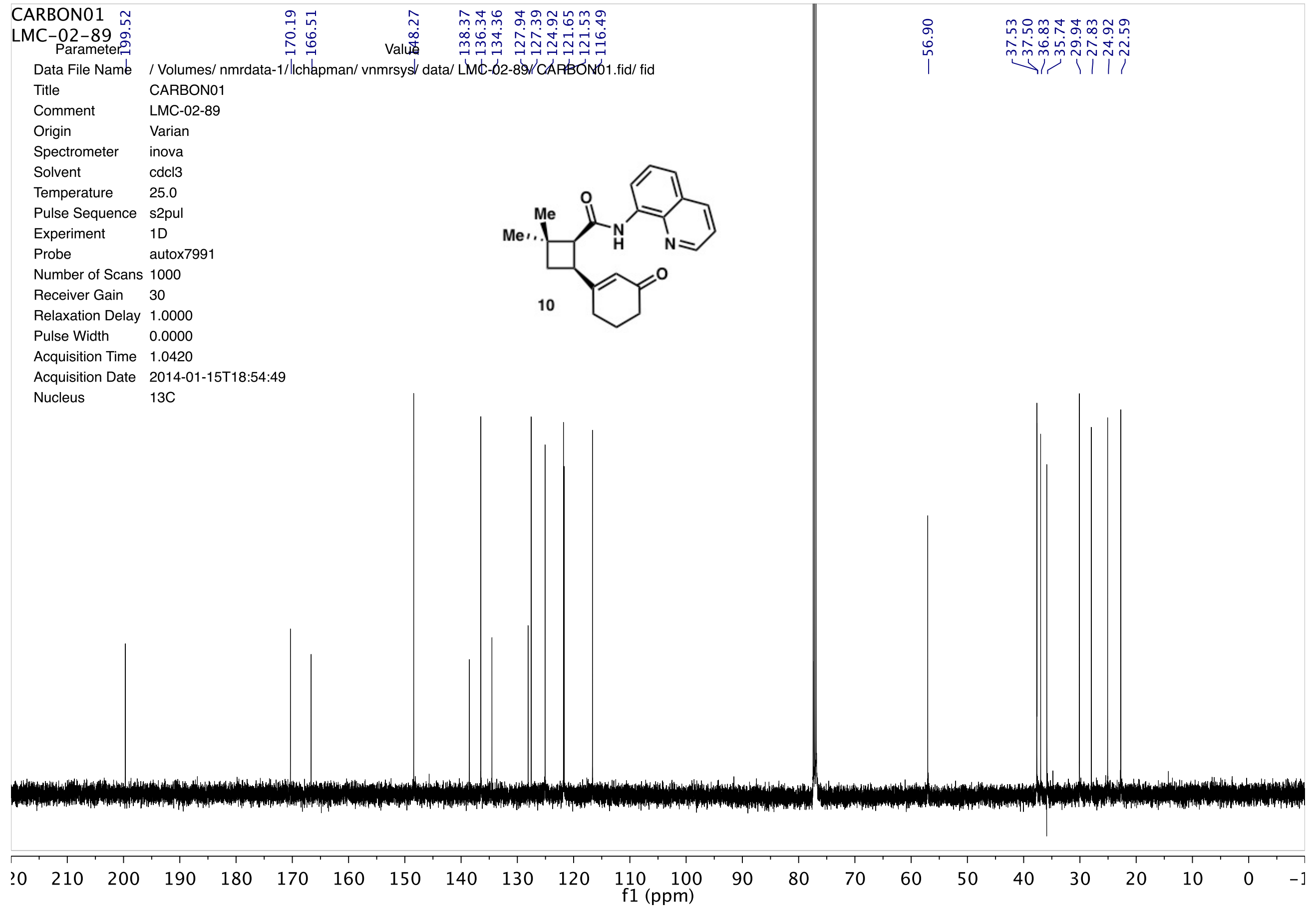




\section{PROTON01}

LMC-pizañeltrans

Value

Data File Name / Volumes/ nmrdata-1/ Ichapman/ vnmrsys/ data/ LMC-02-93trans/ PROTON01.fid/ fid

Title PROTON01

Comment LMC-02-93trans

Origin

Varian

Spectrometer inova

Solvent cdcl3

Temperature $\quad 25.0$

Pulse Sequence s2pul

Experiment 1D

Probe autox7991

Number of Scans 8

Receiver Gain 22

Relaxation Delay 1.0000

Pulse Width $\quad 0.0000$

Acquisition Time 3.0000

Acquisition Date 2014-01-17T12:26:28

Nucleus $\quad 1 \mathrm{H}$

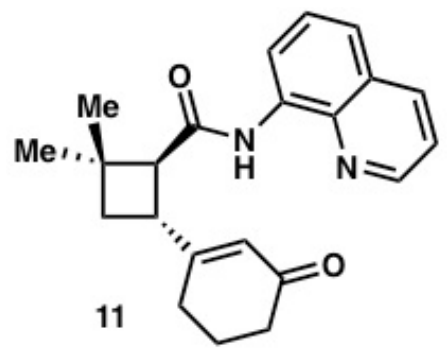

$\mathrm{H}$

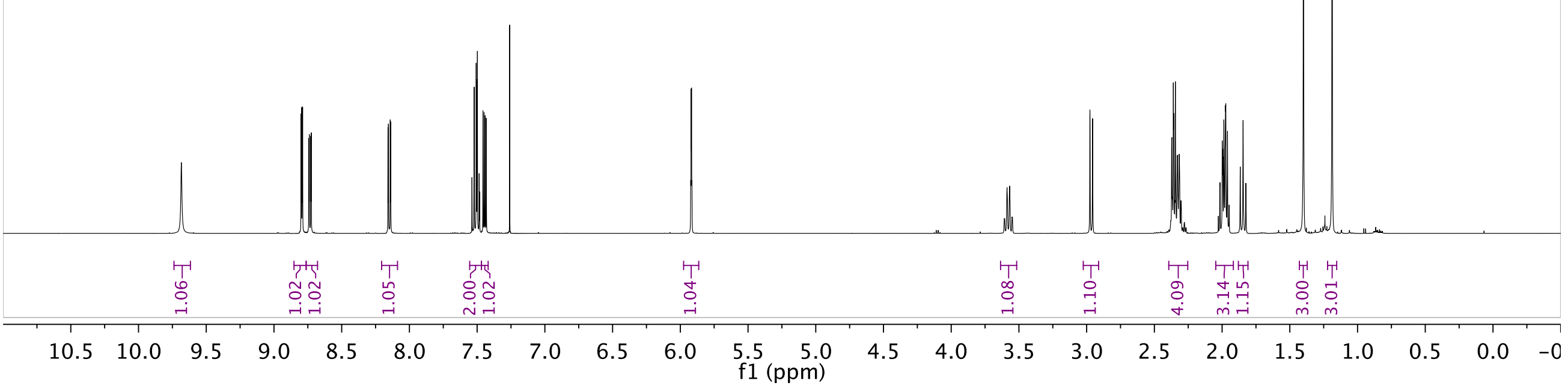




\section{CARBON01 $\underset{\infty}{\infty}$ \\ LMC-pizampetsians

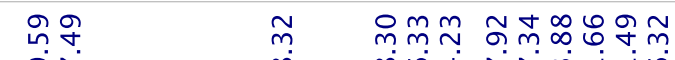

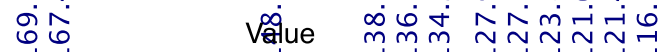

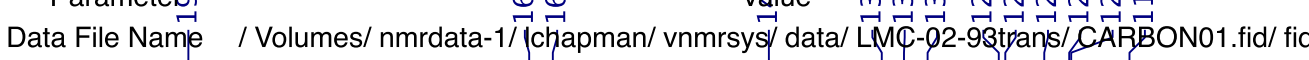

Title

CARBON01

Comment LMC-02-93trans

Origin

Varian

Spectrometer inova

Solvent cdcl3

Temperature 25.0

Pulse Sequence s2pul

Experiment 1D

Probe autox7991

Number of Scans 1000

Receiver Gain 30

Relaxation Delay 0.1000

Pulse Width

0.0000

Acquisition Time 1.0420

Acquisition Date 2014-01-17T12:27:16

Nucleus

$13 \mathrm{C}$

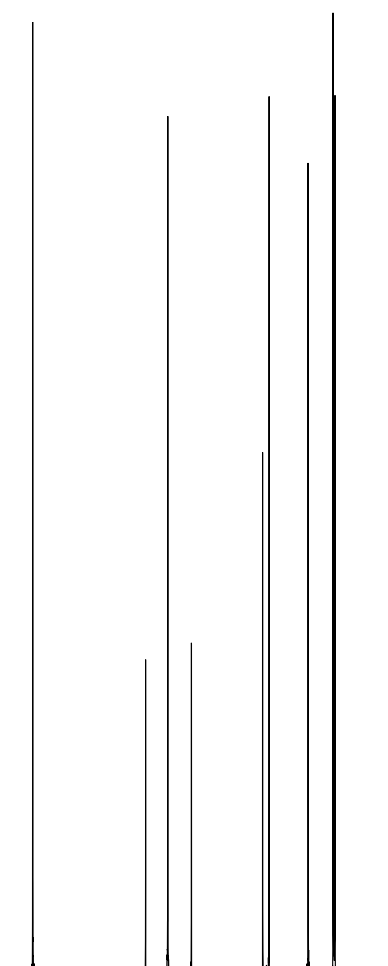

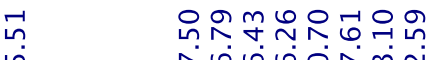

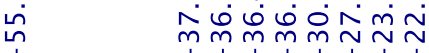

।<smiles>CC1(C)C[C@H](C2=CC(=O)CCC2)[C@H]1C(=O)Nc1cccc2cccnc12</smiles>

11 
LMC-04-128-spiroP1.1.fid

Parameter

Value

Data File Name / Volumes/ nmrdata/ Ichapman/ nmr/ LMC-04-128-spiroP1/ 1/ fid

Title

Volumes/ nmrdata/lchap

Comment

Origin

Bruker BioSpin GmbH

Spectrometer

spect

Solvent

$\mathrm{CDCl} 3$

Temperature

294.9

Pulse Sequence

zg30

Experiment

$1 \mathrm{D}$

Number of Scans 1

Receiver Gain $\quad 30$

Relaxation Delay $\quad 1.0000$

Pulse Width $\quad 11.7000$

Acquisition Time $\quad 4.0894$

Acquisition Date

2016-07-10T16:21:08

Spectrometer Frequency 400.13

Spectral Width $\quad 8012.8$

Nucleus $\quad 1 \mathrm{H}$

Acquired Size $\quad 32768$

Spectral Size $\quad 65536$

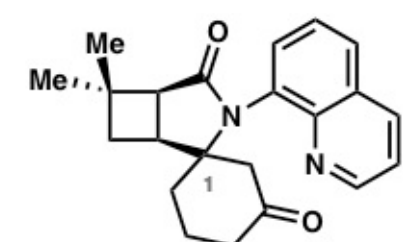

s1

2.5:1 mixture of

diastereomers at C1)

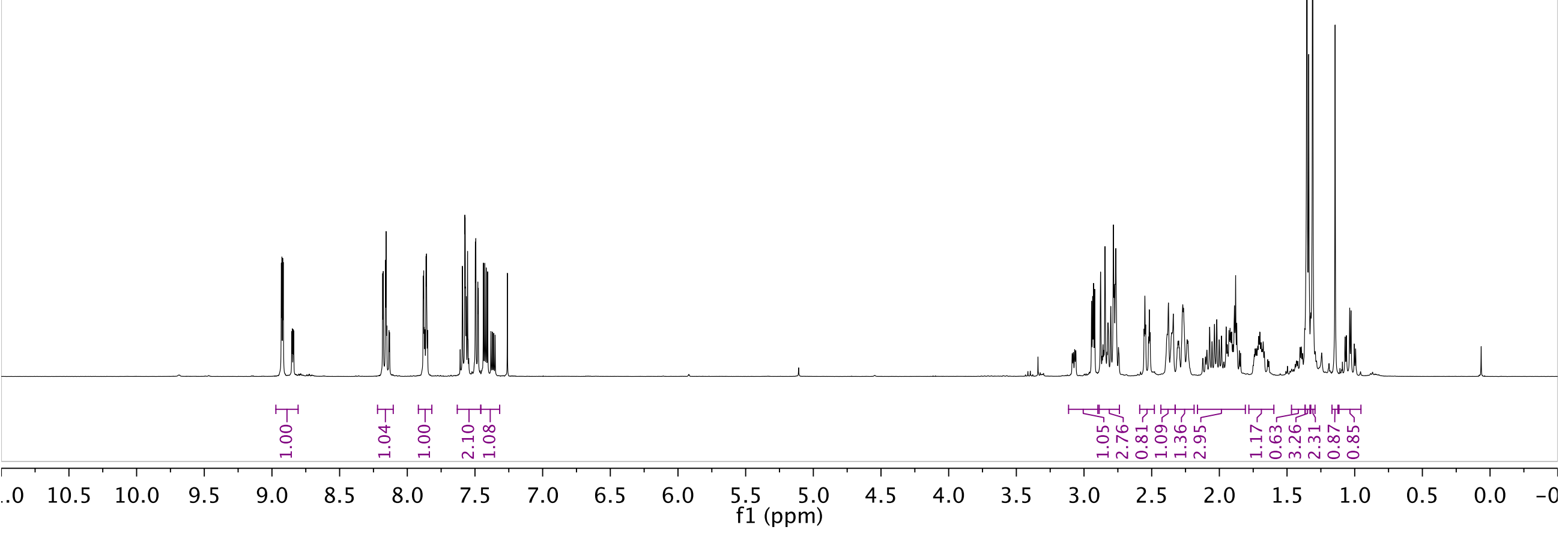


O) ÓParameter

Data File Name

Title

Comment

Origin

Spectrometer

Solvent

Temperature

Pulse Sequence

Experiment

Number of Scans

Receiver Gain

Relaxation Delay

Pulse Width

Acquisition Time

Acquisition Date

Spectrometer Freq

Spectral Width

Nucleus

Acquired Size

Spectral Size

/ Volumes/

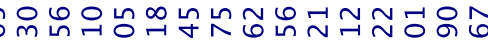

Valué 守

LMC-04-128-spiroP1.2.fid

Bruker BioSpin GmbH

spect

$\mathrm{CDCl} 3$

294.9

zgpg30

$1 \mathrm{D}$

128

72

2.0000

10.0000

1.3631

2016-07-10T16:29:05

00.62

24038.5

$13 \mathrm{C}$

32768

65536

diastereomers at $\mathrm{C} 1$

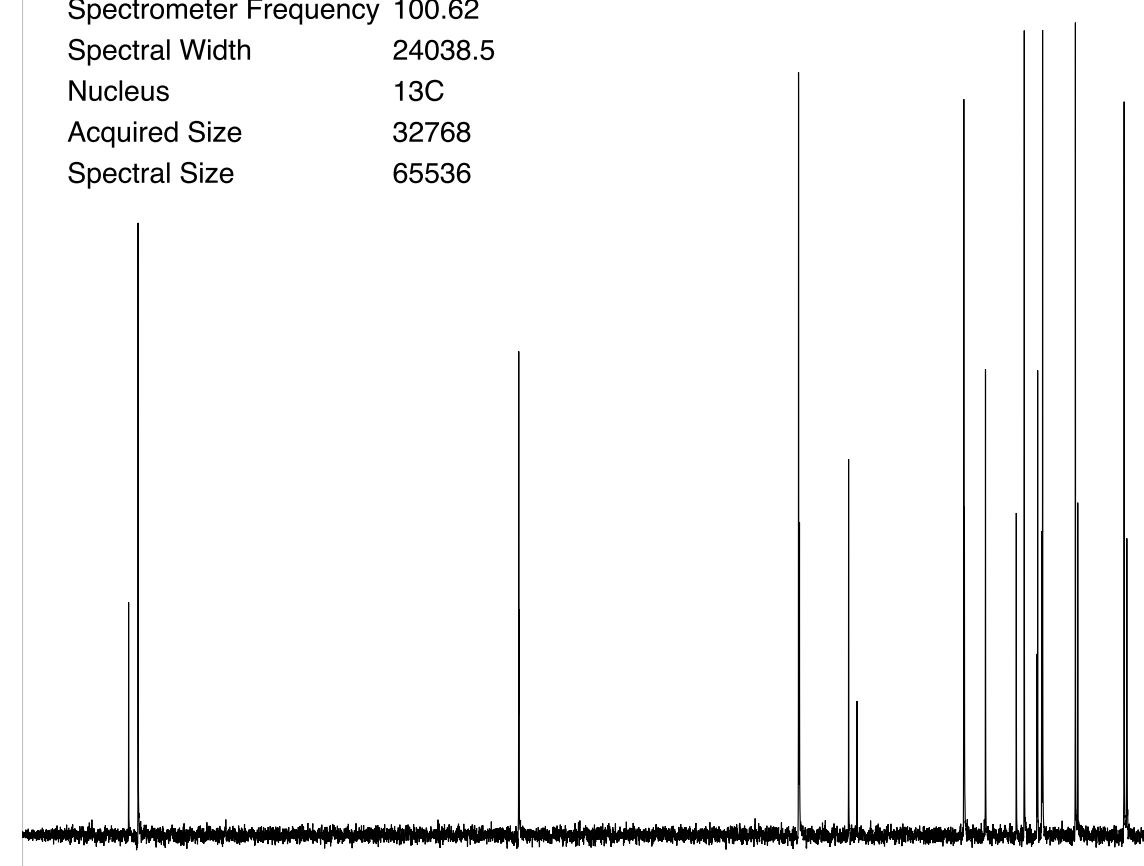

(2.5:1 mixture

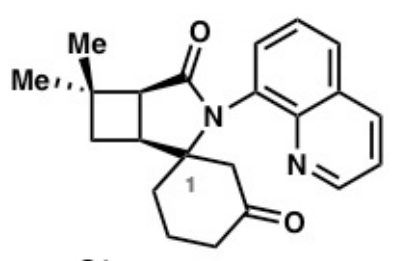

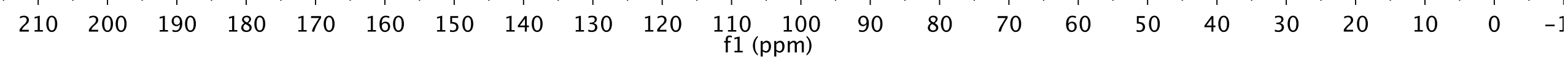


LMC-04-107-P1.1.fid

Parameter

Data File Name

Title

Origin

Solvent

Temperature

Pulse Sequence

Number of Scans

Receiver Gain

Relaxation Delay

Pulse Width

Acquisition Time

Acquisition Date

Spectrometer Fr

Spectral Width

Lowest Frequency

Nucleus

Acquired Size

Spectral Size
Value

/ Volumes/ nmrdata/ Ichapman/ nmr/ LMC-04-107-P1/ 1/ fid

LMC-04-107-P1.1.fid

Bruker BioSpin GmbH

$\mathrm{CDCl} 3$

294.9

zg30

1.0000

11.7000

4.0894

2016-03-10T14:57:25

400.13

8012.8

$-1555.6$

$1 \mathrm{H}$

32768

65536
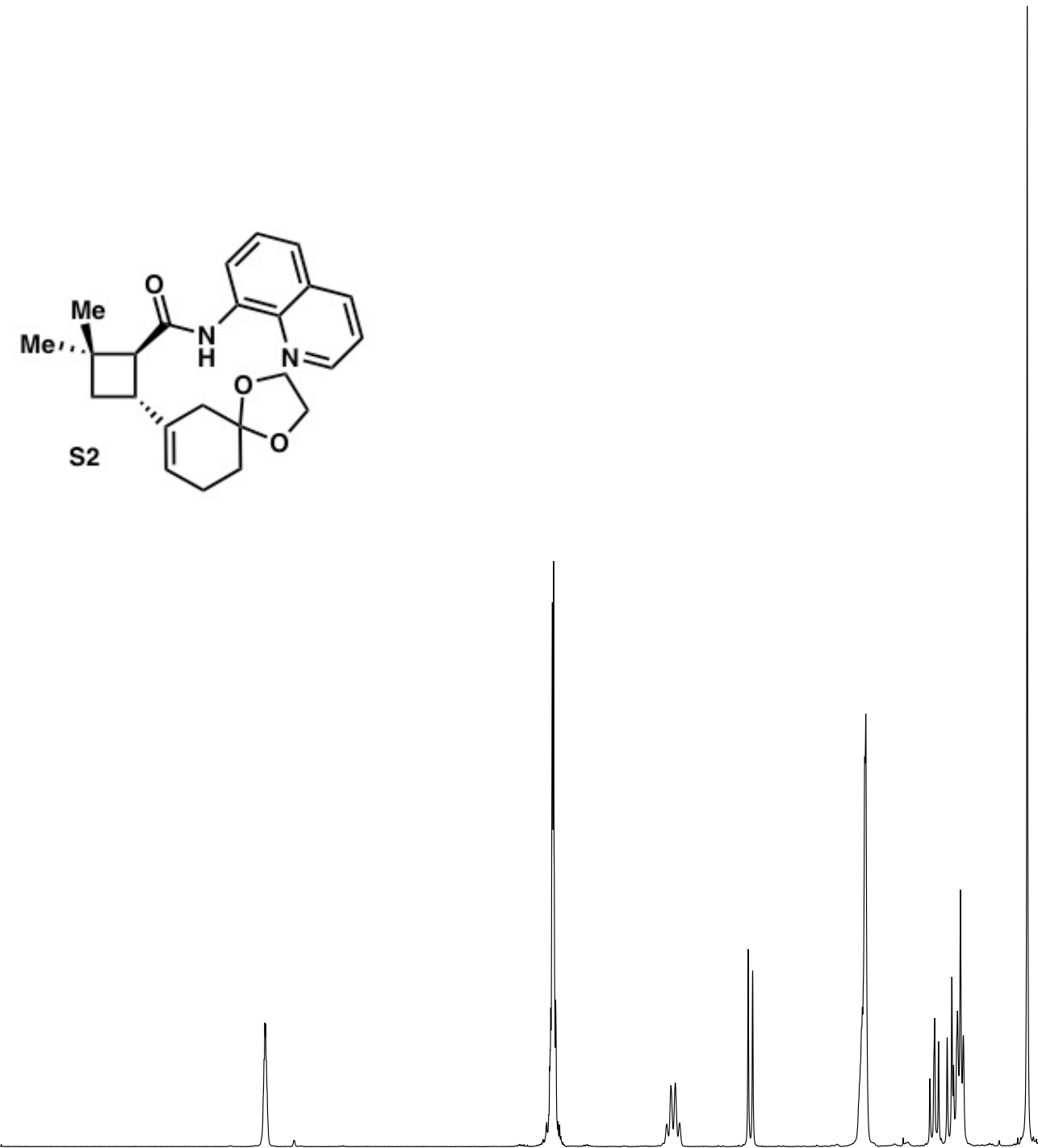

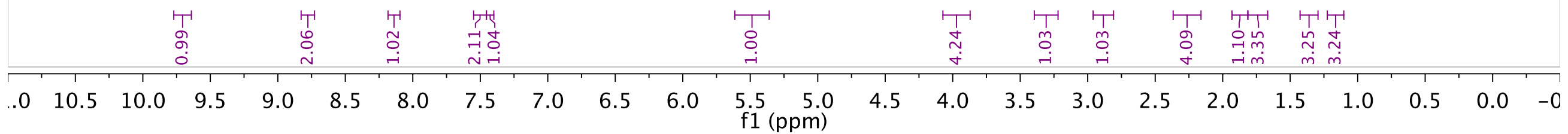




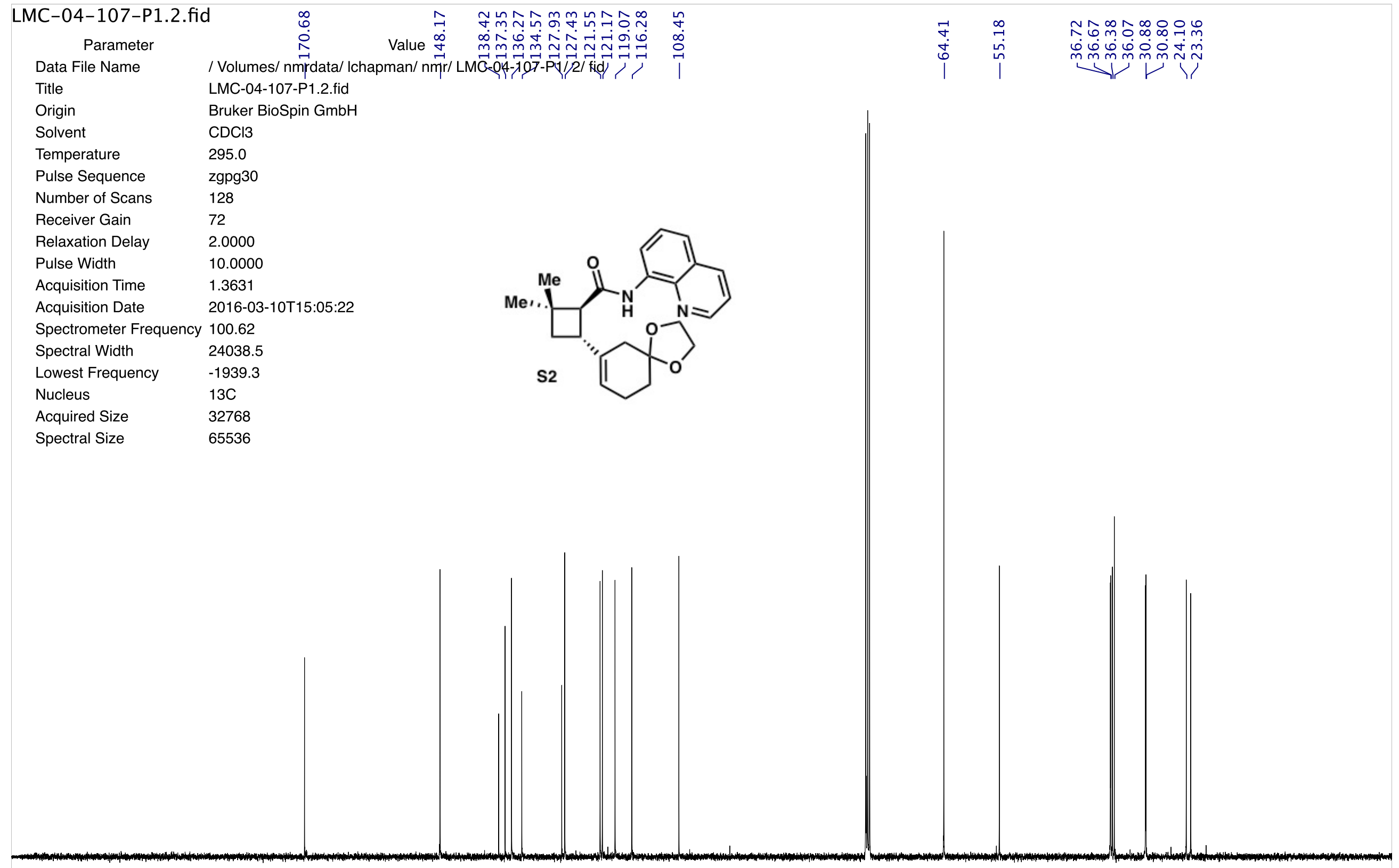

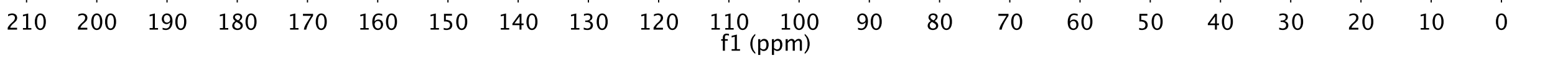


Data File Name / Volumes/nmrdata-1/ Ichapman/ vnmrsys/ data/ LMC-04-129/ PROTON01.fid/ fid

Title PROTON01

Comment LMC-04-129

Origin

Varian

Spectrometer

inova

Solven

cdcl3

25.0

Pulse Sequence s2pul

Experiment 1D

Probe autox7991

Number of Scans 1

Receiver Gain 22

Relaxation Delay 1.0000

Pulse Width

0.0000

Acquisition Time 3.0000

Acquisition Date 2016-06-13T20:55:49

Nucleus

mixture as used in Schwartz reduction
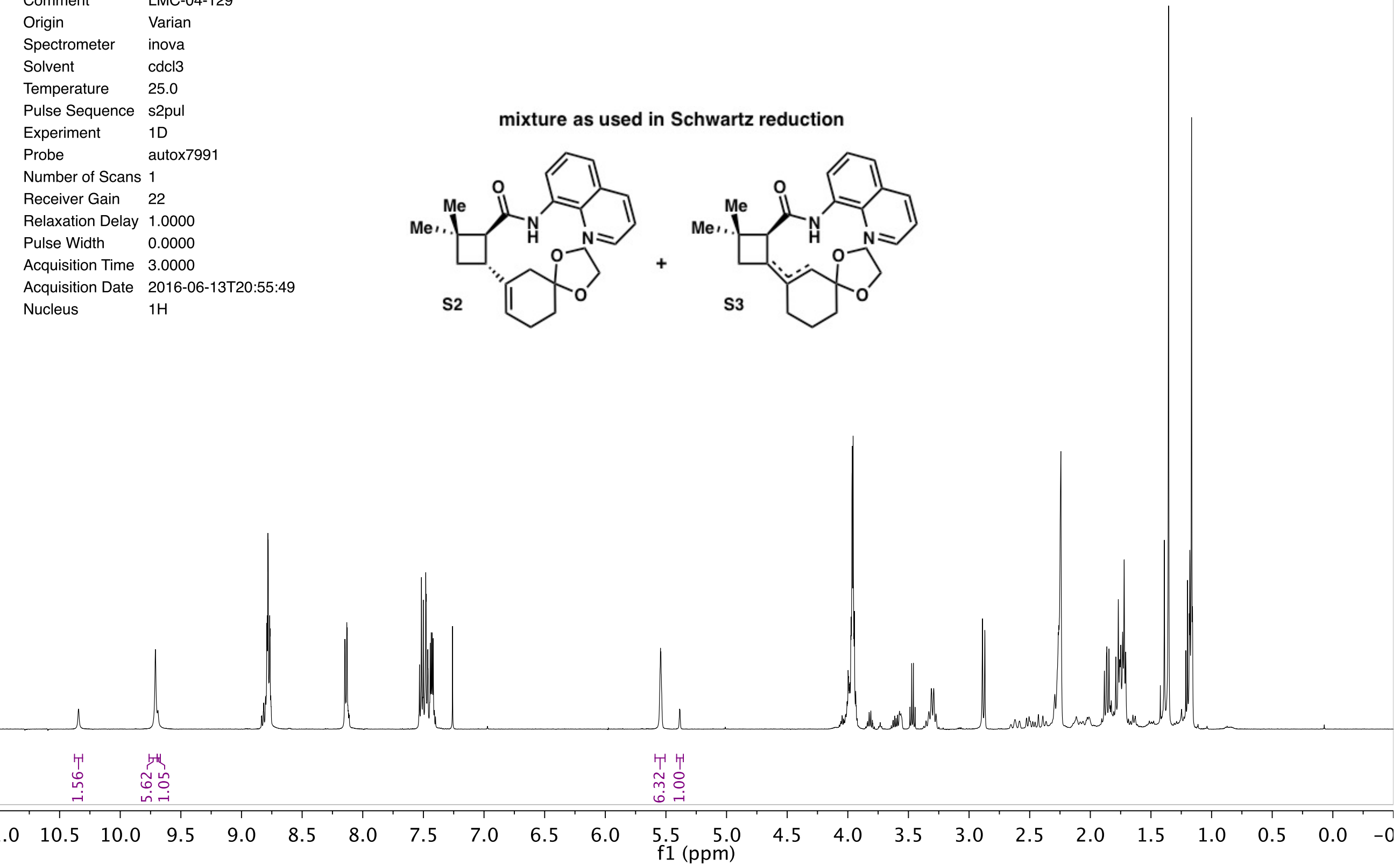


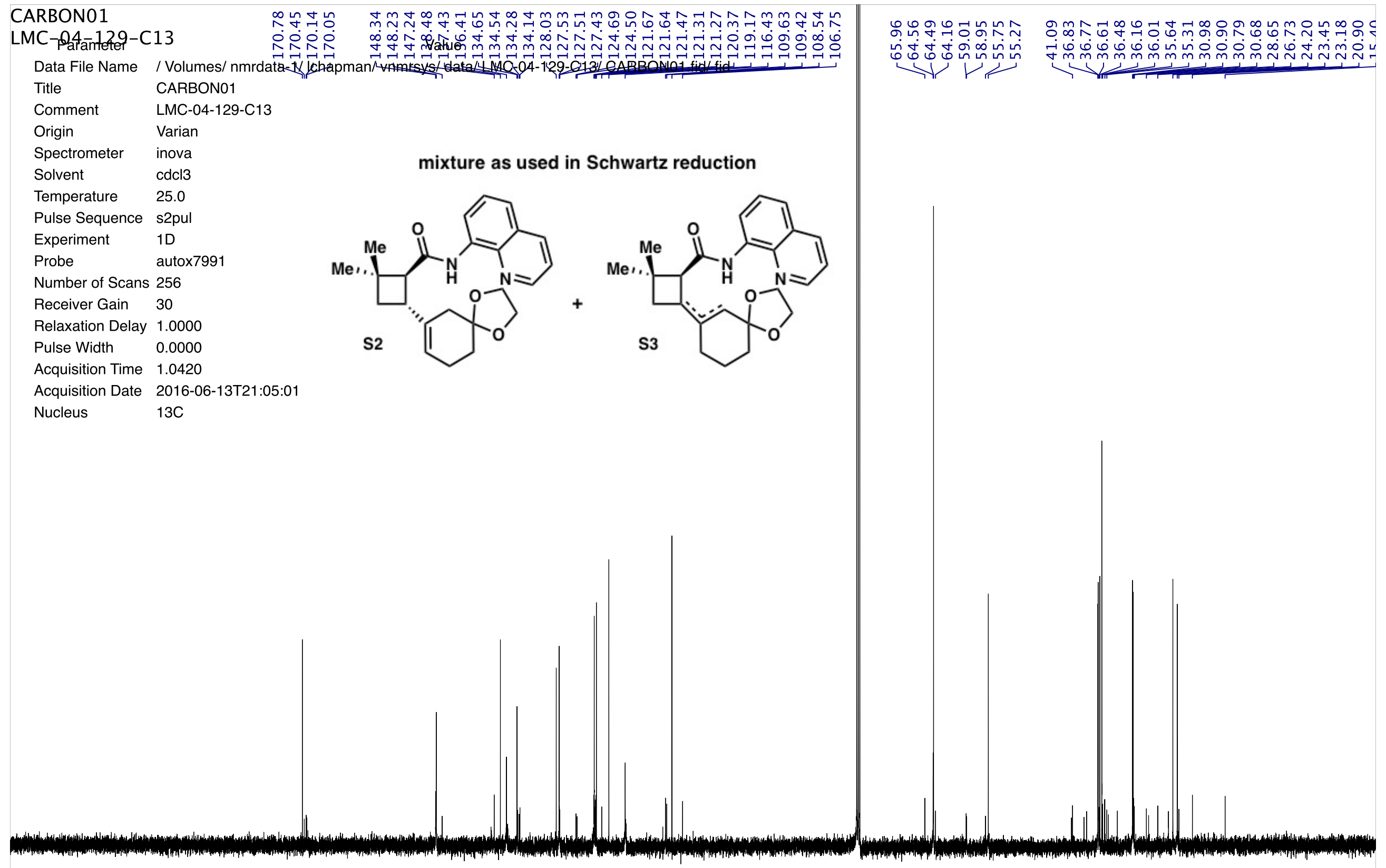

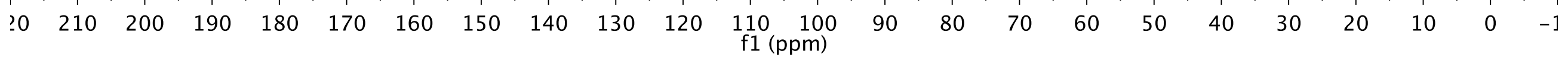


LMC-04-113.1.fid

Parameter

Data File Name

/ Volumes/ nmrdata/ Ichapman/ nmr/ LMC-04-113/ 1/ fid

Title

LMC-04-113.1.fid

Origin

Bruker BioSpin GmbH

Solvent

$\mathrm{CDCl} 3$

Temperature

294.9

Pulse Sequence $\quad$ zg30

Number of Scans 1

Receiver Gain $\quad 30$

Relaxation Delay $\quad 1.0000$

Pulse Width $\quad 11.7000$

Acquisition Time $\quad 4.0894$

Acquisition Date 2016-03-15T18:02:01

Spectrometer Frequency 400.13

Spectral Width

8012.8

Lowest Frequency

Nucleus

$-1545.4$

Acquired Size

Spectral Size $\quad 65536$
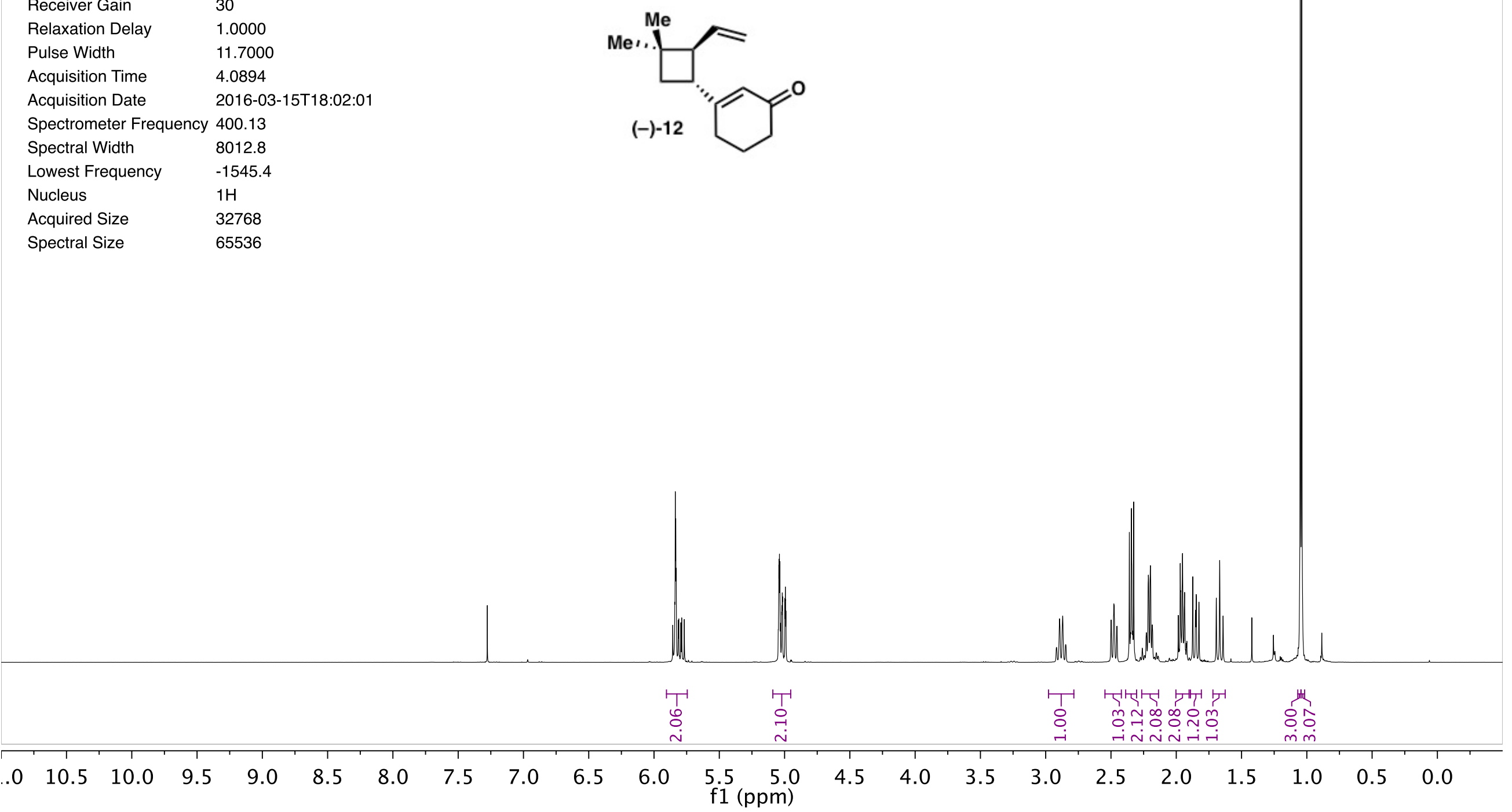


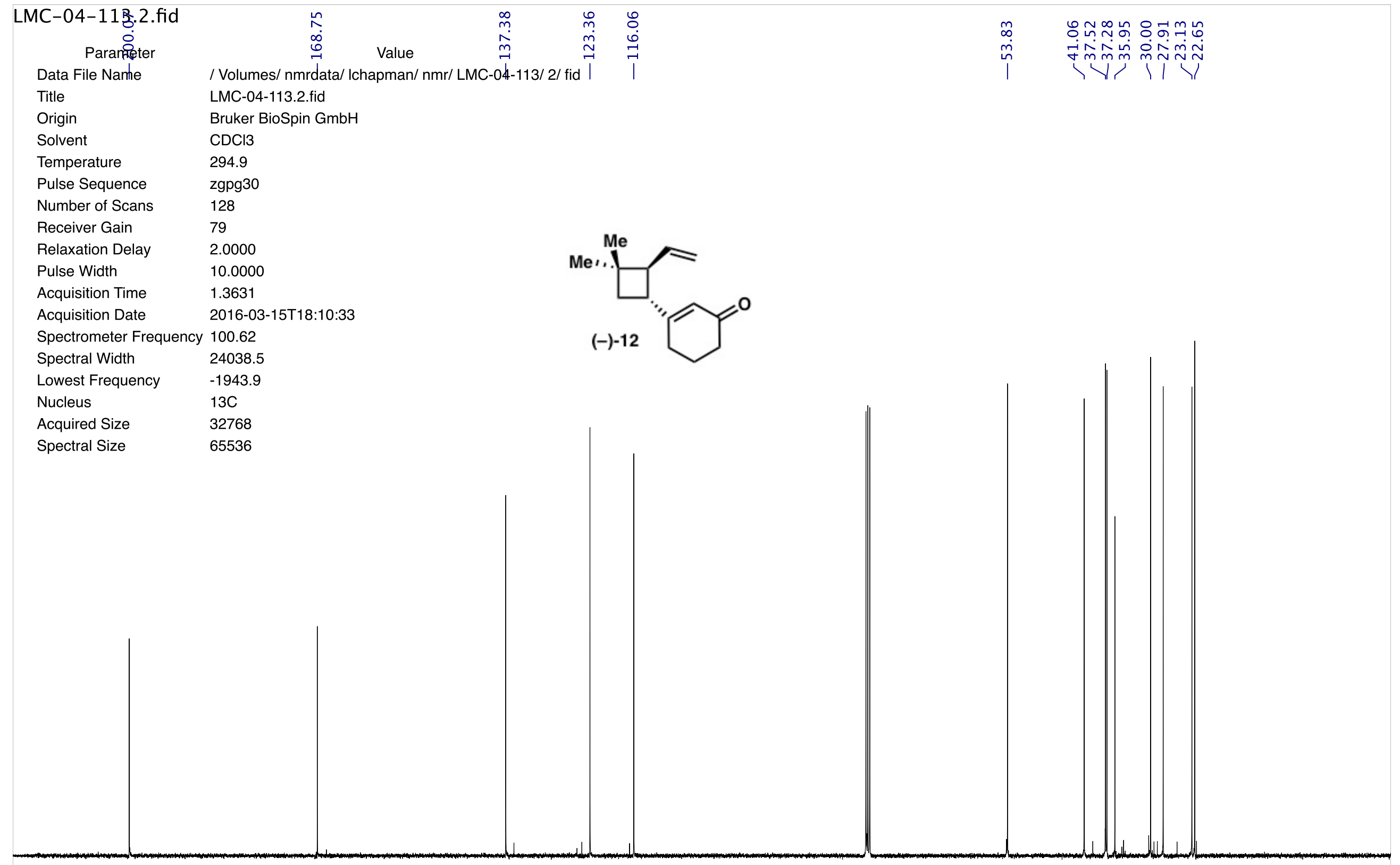

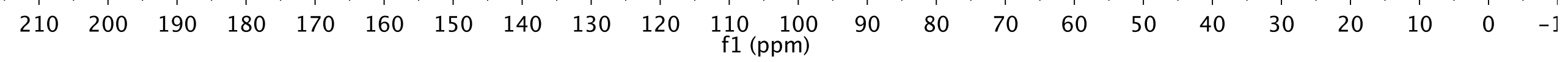




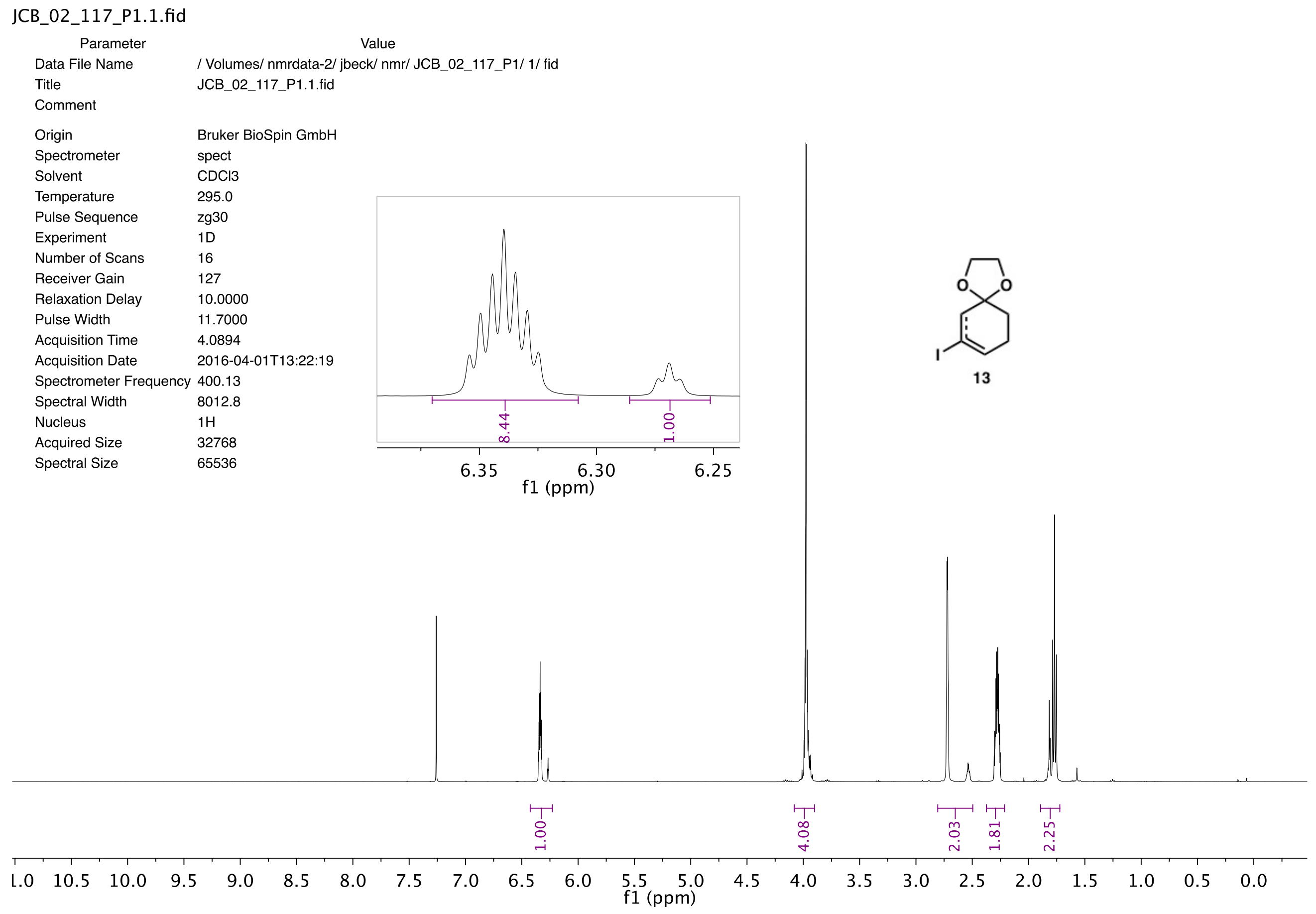




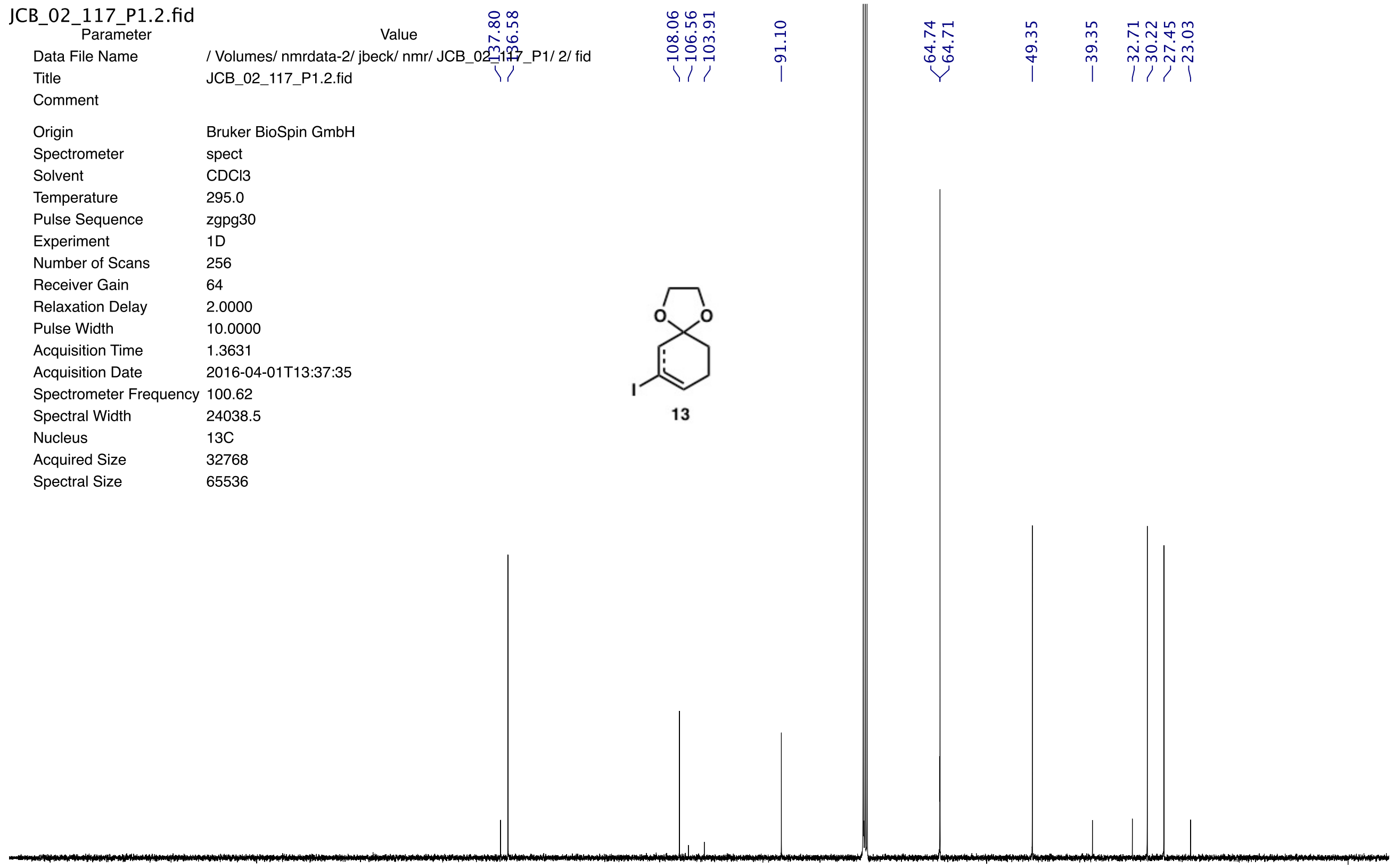

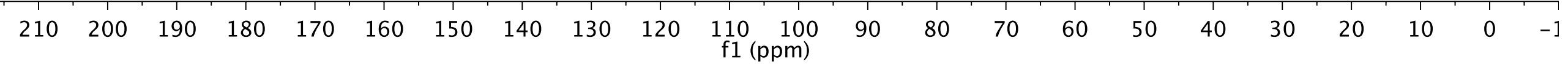




\section{JCB_02_211_P2.1.fid}

Parameter

Value

Data File Name

/ Volumes/ nmrdata/ jbeck/ nmr/JCB_02_211_P2/ 1/ fid

Title

JCB 02 211_P2.1.fid

Comment

Origin

Bruker BioSpin GmbH

Spectrometer

spect

Solvent

$\mathrm{CDCl} 3$

Temperature

295.0

zg30

Experiment

Number of Scans

Receiver Gain

Relaxation Delay

$1 \mathrm{D}$

16

Pulse Width

Acquisition Time

11.7000

Acquisition Date

2016-06-03Т20:57:52

Spectrometer Frequency 400.13

Spectral Width $\quad 8012.8$

Nucleus $1 \mathrm{H}$

Acquired Size $\quad 32768$

Spectral Size $\quad 65536$
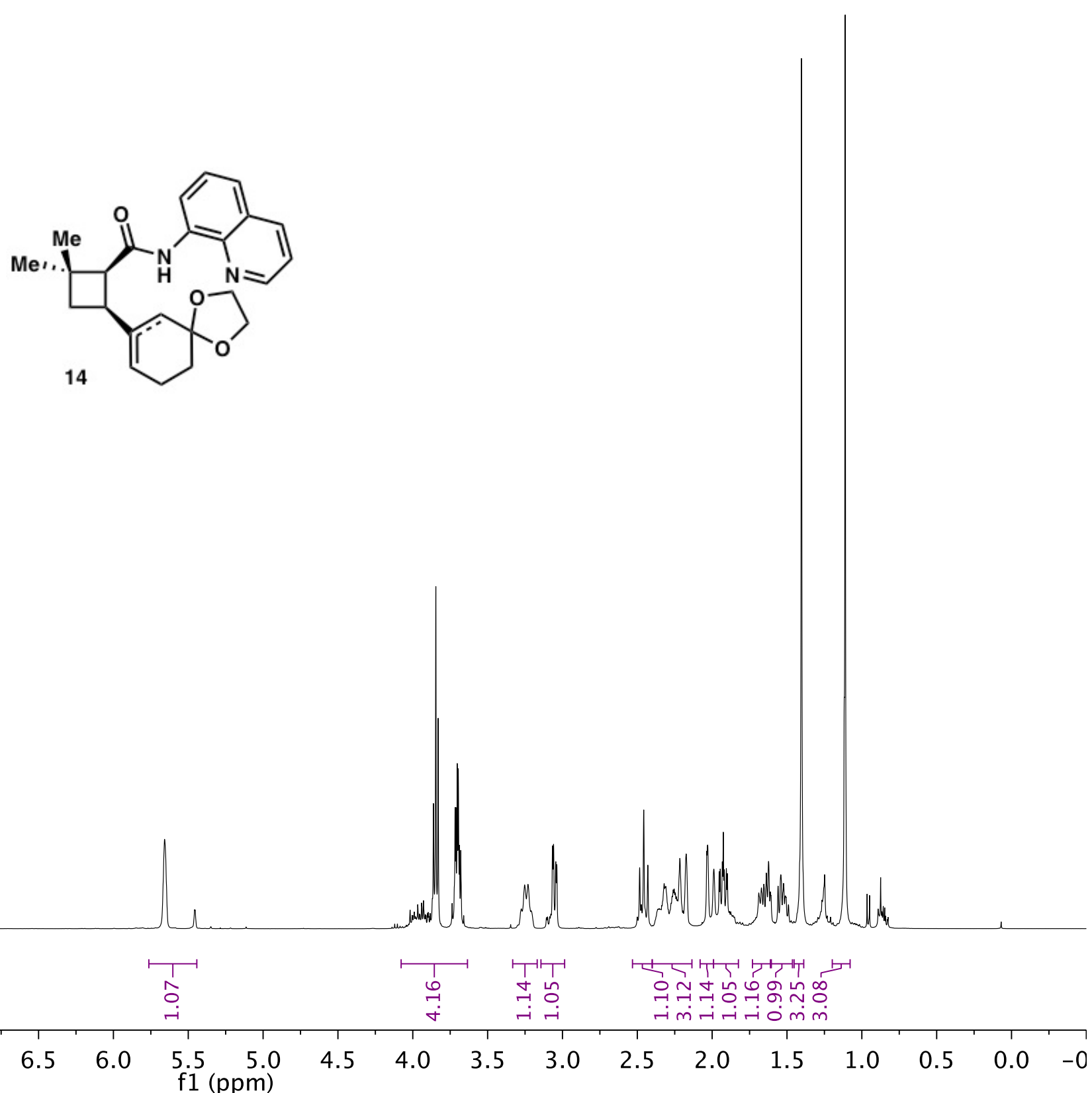


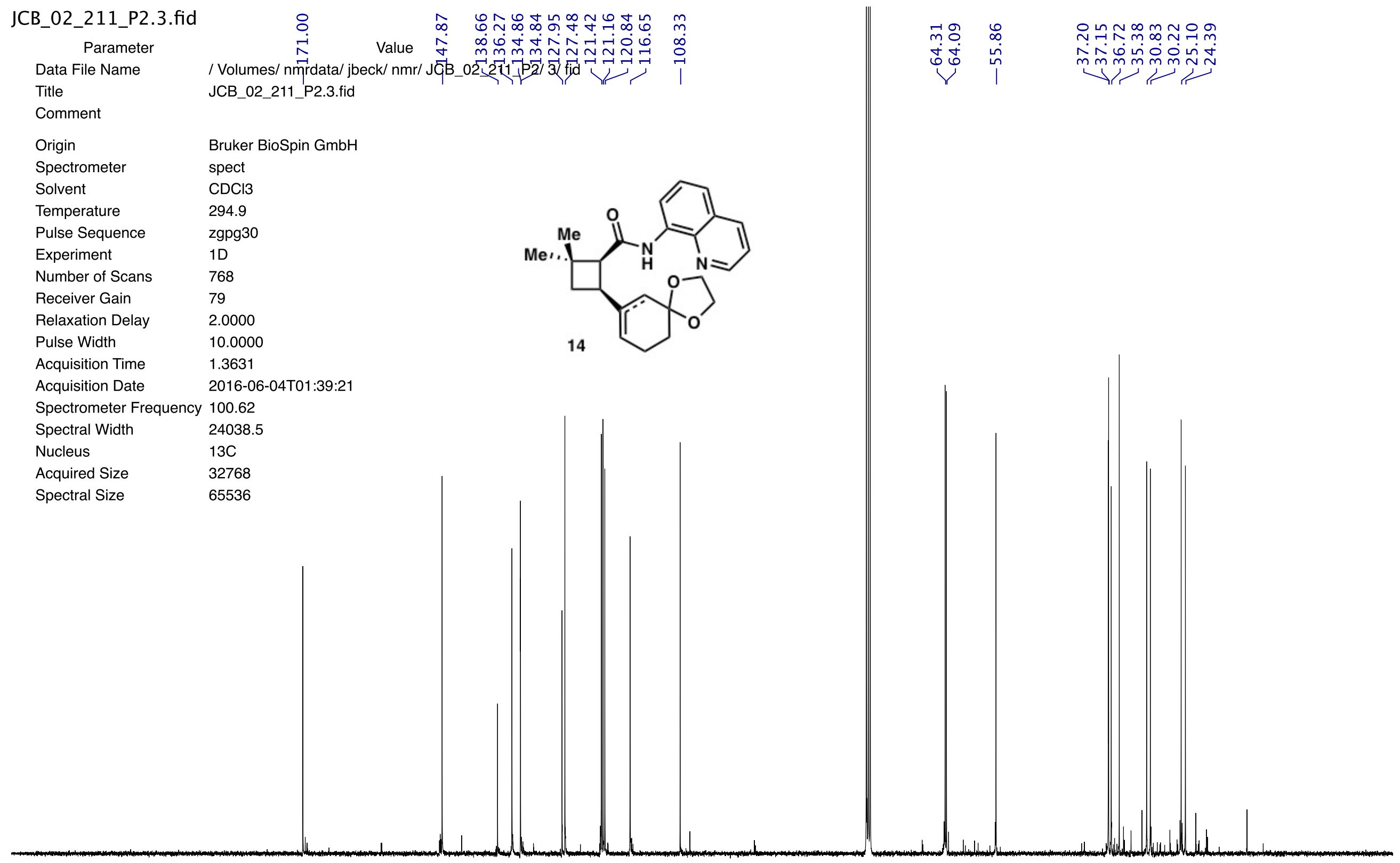

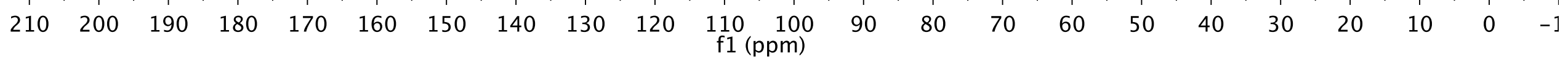




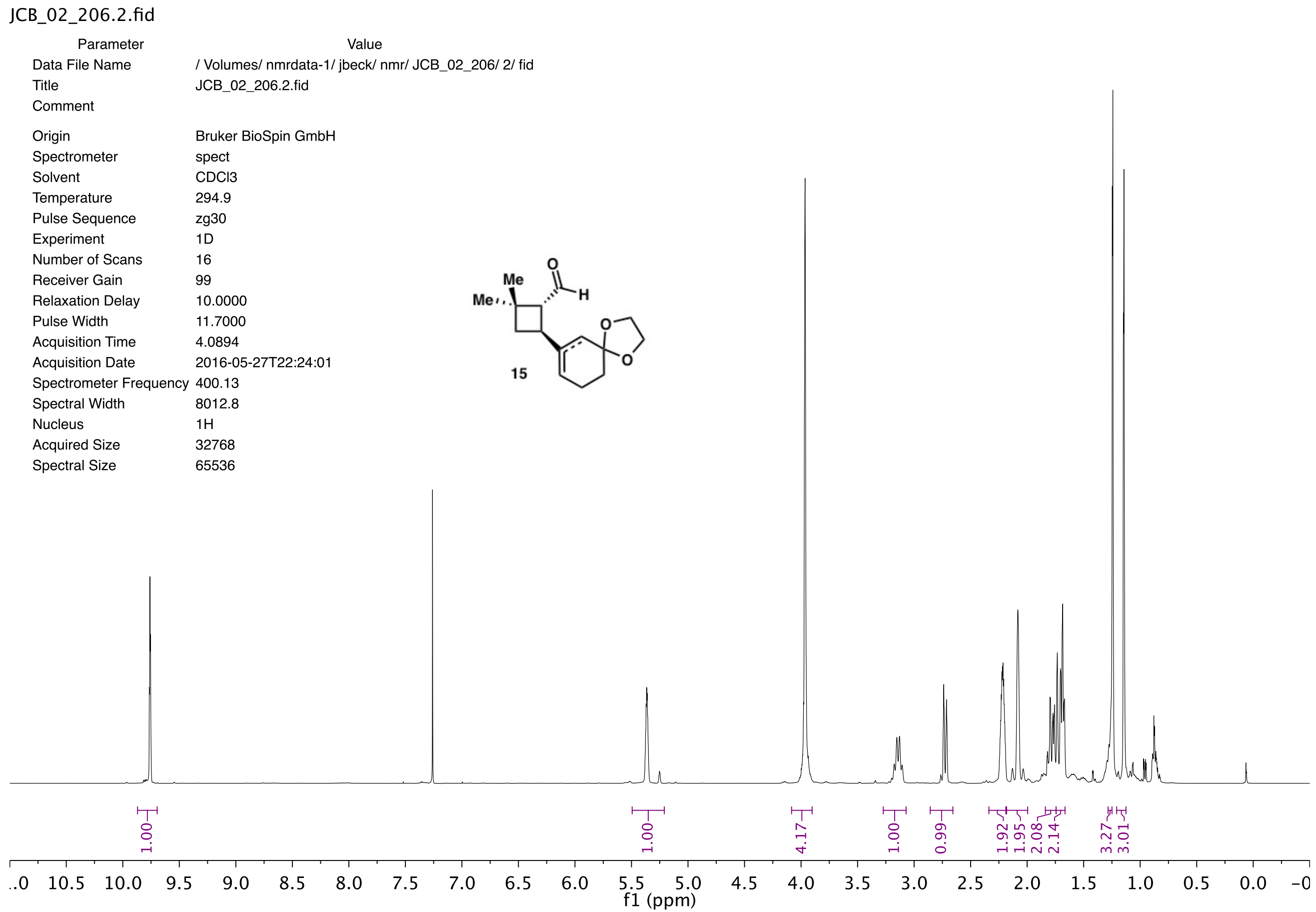




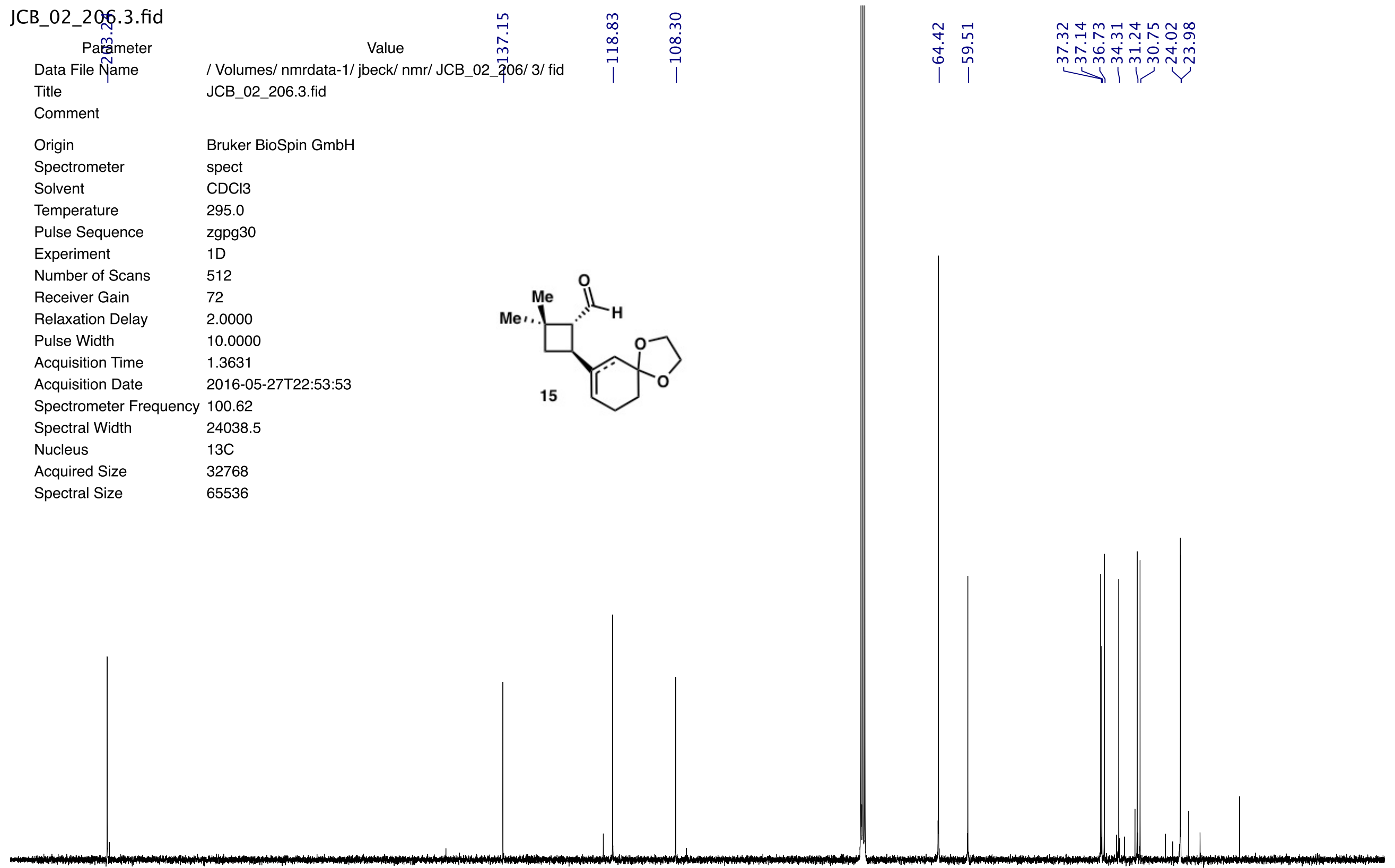

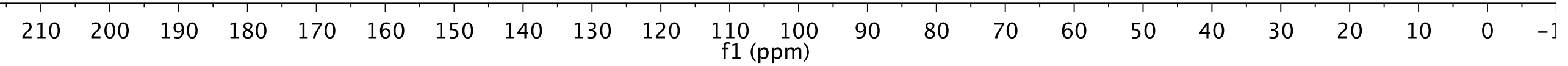




\section{JCB-02-207-vinylenone.1.fid}

Parameter Data File Name

Title

Comment

Origin

Spectrometer

Solvent

Temperature

Pulse Sequence

Experiment

Number of Scans

Receiver Gain

Relaxation Delay

Pulse Width

Acquisition Time

Acquisition Date

Spectrometer Freq

Spectral Width

Nucleus

Acquired Size

Spectral Size
Value

/ Volumes/ nmrdata/ Ichapman/ nmr/ JCB-02-207-vinylenone/ 1/ fid

JCB-02-207-vinylenone.1.fid

Bruker BioSpin GmbH

spect

$\mathrm{CDCl} 3$

295.0

$\mathrm{zg} 30$

16

79

10.0000

11.7000

2016-05-28T20:39:59

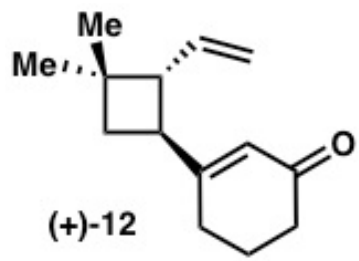

80128

32768

65536

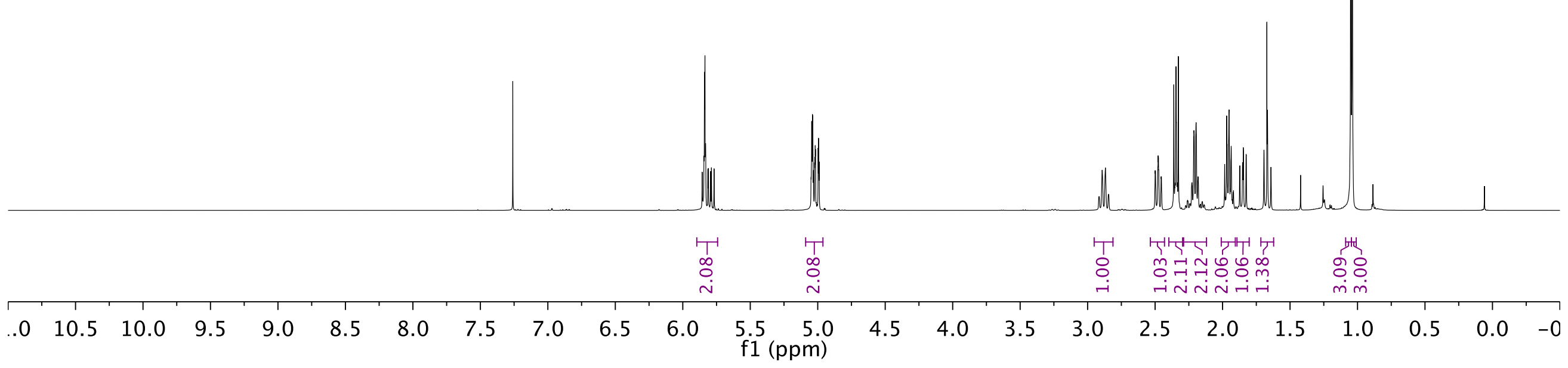


JCB-02-207@vinylenone.2.fid

$$
\text { Paranister }
$$

Data File Name

Title

Comment

Origin

Spectrometer

Solvent

Temperature

Pulse Sequence

Experiment

Number of Scans

Receiver Gain

Relaxation Delay

Pulse Width

Acquisition Time

Acquisition Date

Spectrometer Freq

Spectral Width

Nucleus

Acquired Size

Spectral Size

spect

$\mathrm{CDCl} 3$

295.0

zgpg30

$1 \mathrm{D}$

1024

2.0000

10.0000

1.3631

24038.5

$13 \mathrm{C}$

32768

65536

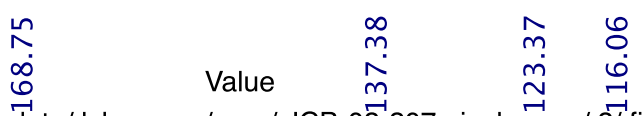

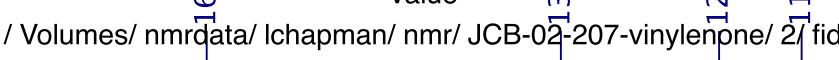
JCB-02-207-vinylenone.2.fid

Bruker BioSpin GmbH

2016-05-28T21:39:07

$(+)-12$

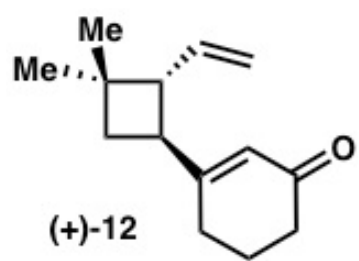

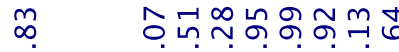

ñ

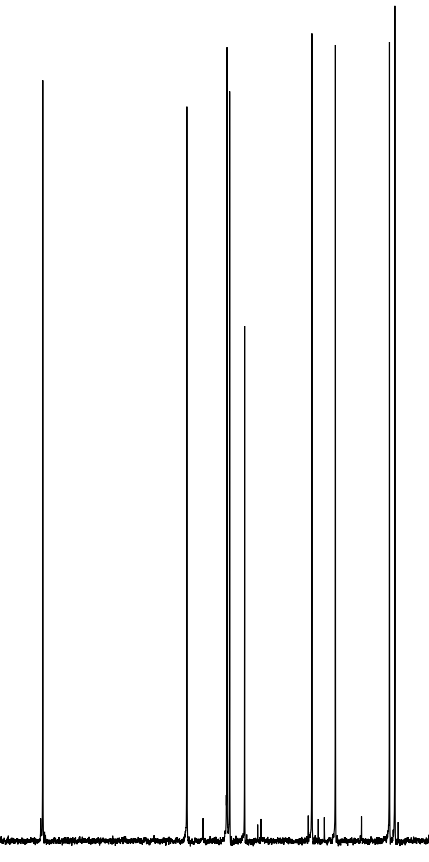

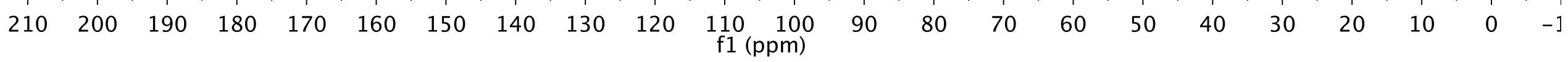


LMC-04-186-C6D6.1.fid

Parameter

Value

Data File Name / Volumes/ nmrdata/ Ichapman/ nmr/ LMC-04-186-C6D6/ 1/ fid

Title

LMC-04-186-C6D6.1 fid

Origin Bruker BioSpin $\mathrm{GmbH}$

Solvent $\quad \mathrm{CDCl} 3$

Temperature 295.0

Pulse Sequence $\quad$ zg30

Number of Scans 1

Receiver Gain 30

Relaxation Delay $\quad 1.0000$

Pulse Width $\quad 11.7000$

Acquisition Time $\quad 4.0894$

Acquisition Date 2016-05-30T21:05:59

Spectrometer Frequency 400.13

Spectral Width $\quad 8012.8$

Lowest Frequency $\quad-1564.4$

Nucleus $\quad 1 \mathrm{H}$

Acquired Size $\quad 32768$

Spectral Size $\quad 65536$

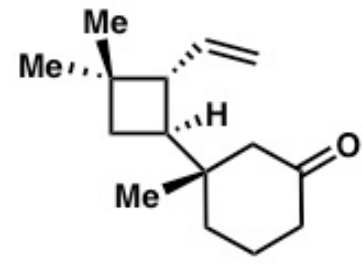

4 (in $\mathrm{C}_{6} \mathrm{D}_{6}$ )
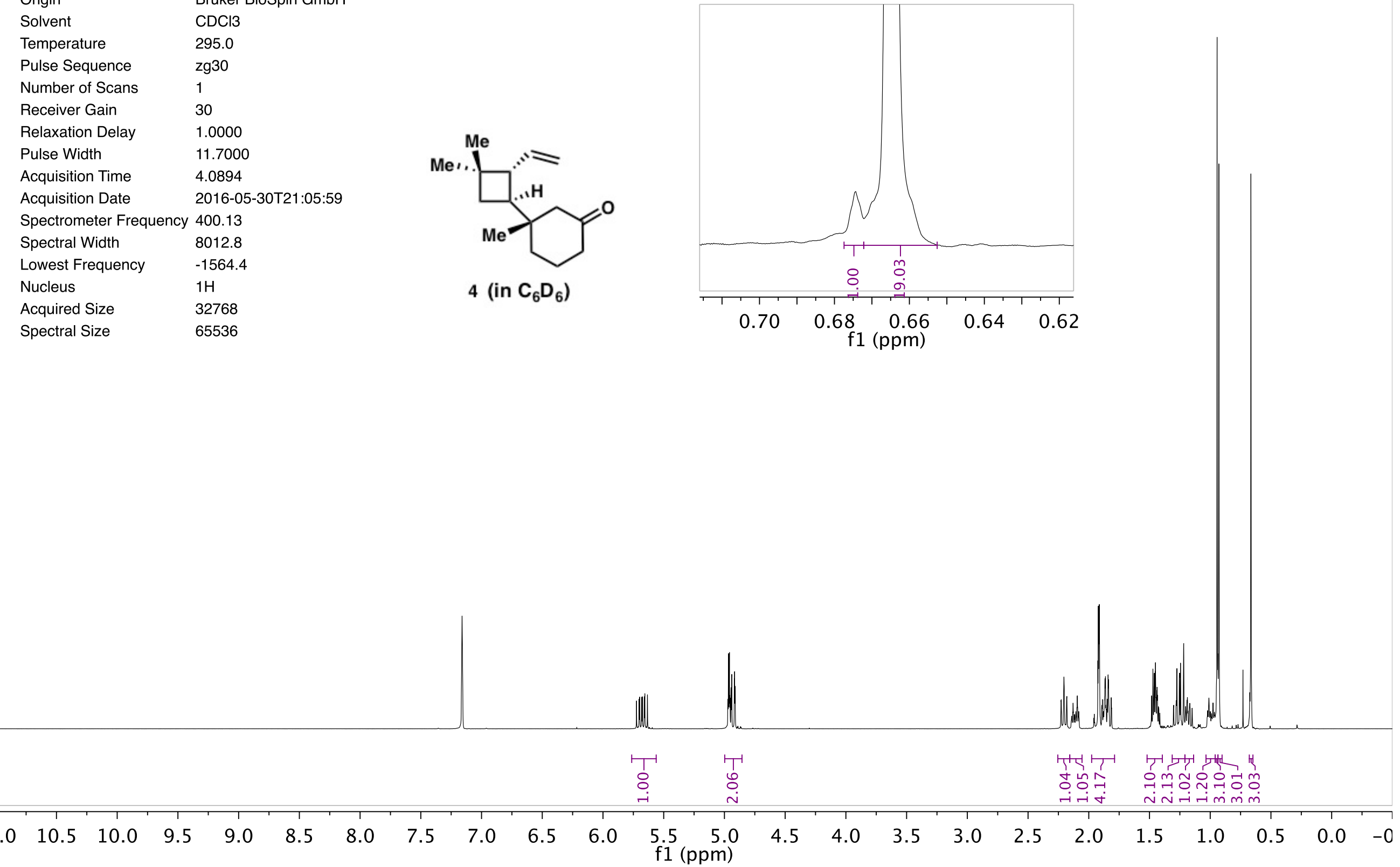


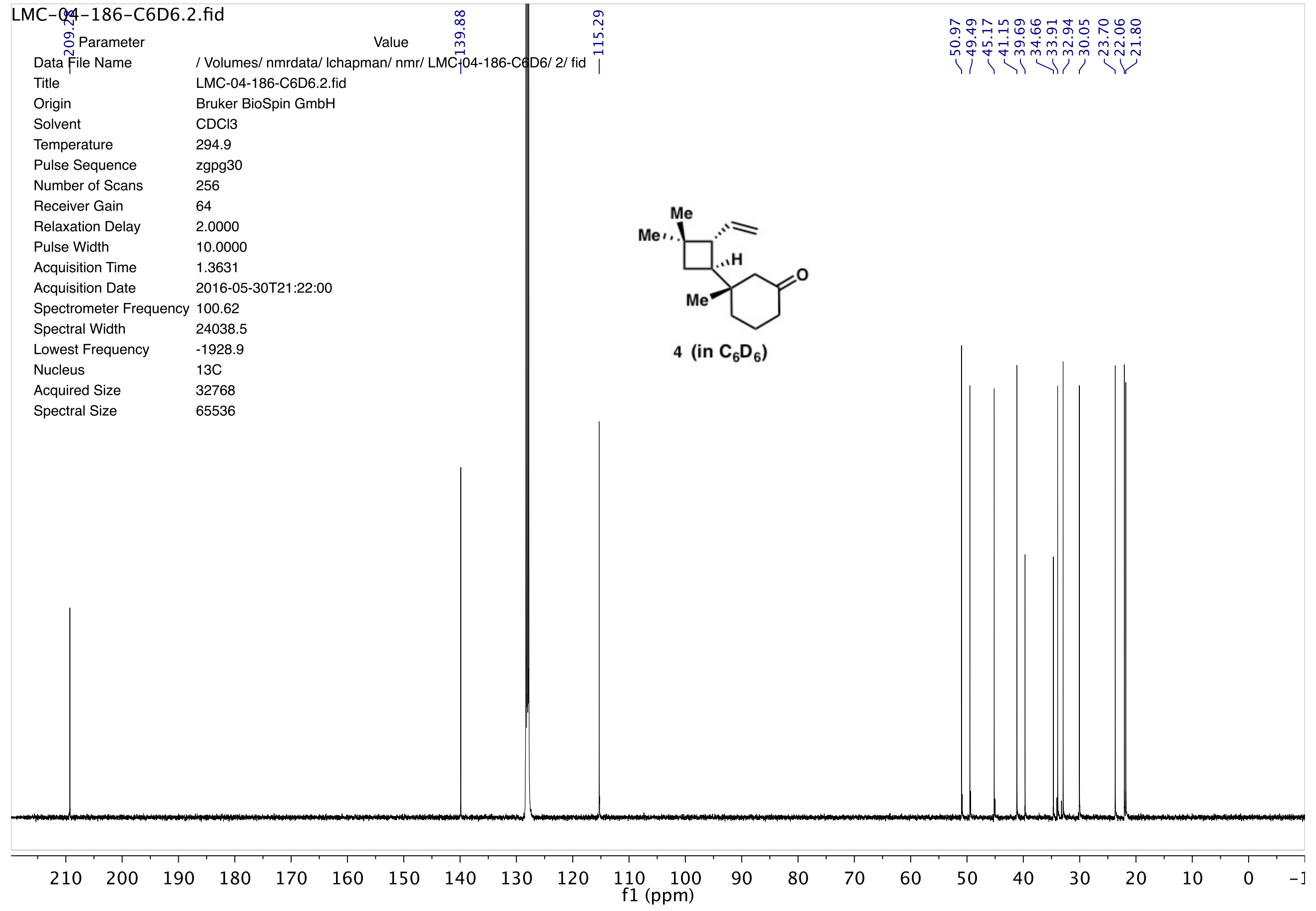


LMC-04-186-vinylketone.2.fid

Parameter

Data File Name

Title

Origin

Solvent

Temperature

Pulse Sequence

Number of Scans

Receiver Gain

Relaxation Delay

Pulse Width

Acquisition Time

Acquisition Date

Spectrometer Frequ

Spectral Width

Lowest Frequency

Nucleus

Acquired Size

Spectral Size
Value

/ Volumes/ nmrdata/ Ichapman/ nmr/ LMC-04-186-vinylketone/ 2/ fid

LMC-04-186-vinylketone.2.fid

Bruker BioSpin GmbH

$\mathrm{CDCl} 3$

295.0

zg30

10.0000

11.7000

4.0894

2016-05-29T20:46:17

400.13

8012.8

$-1545.6$

$1 \mathrm{H}$

32768

65536

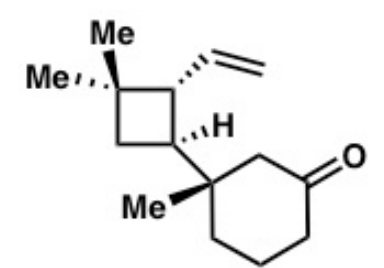

4 (in $\mathrm{CDCl}_{3}$ )

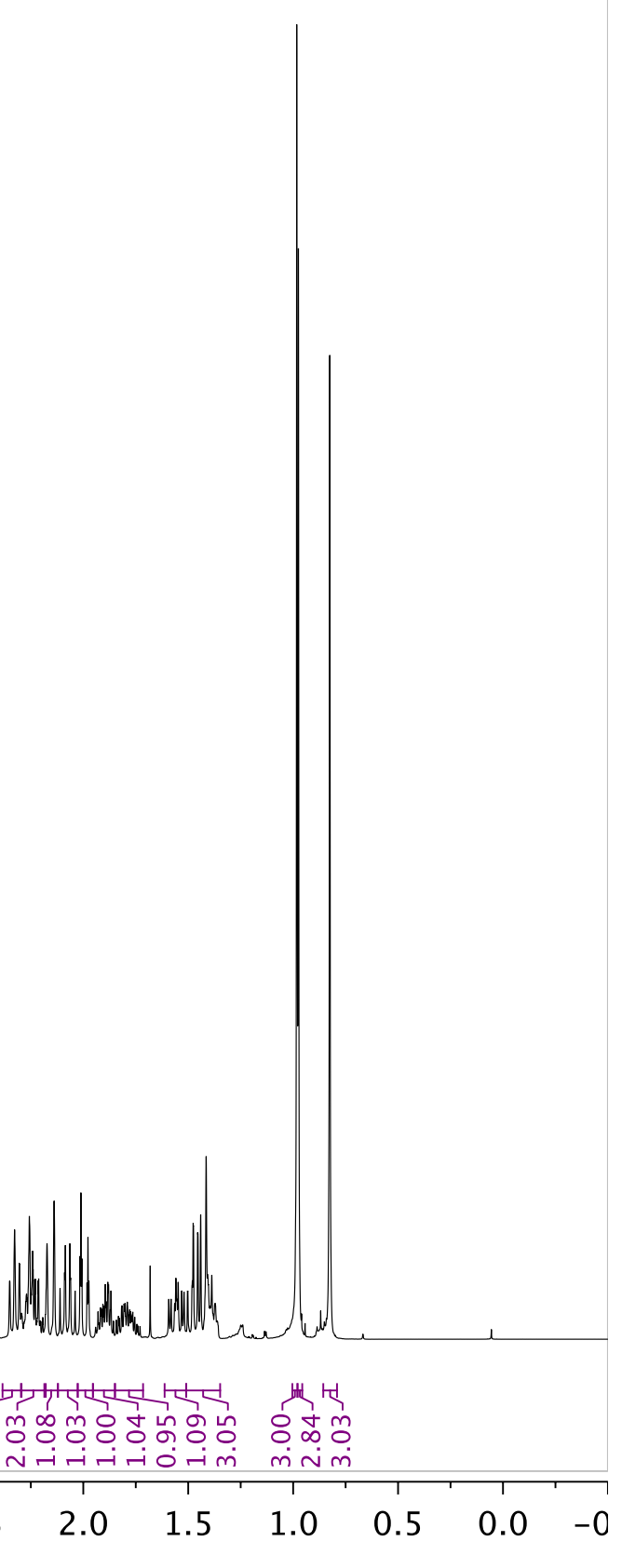




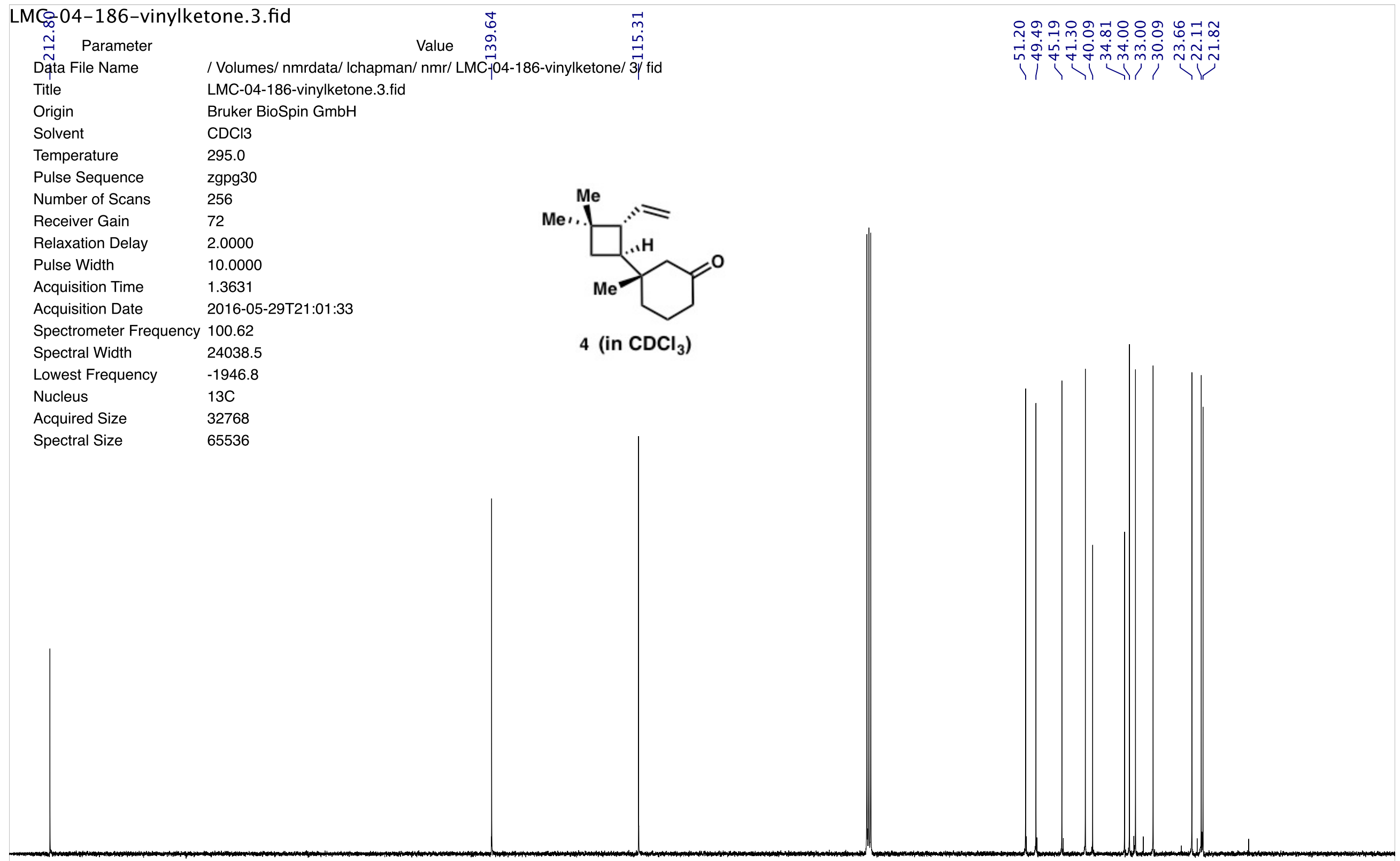

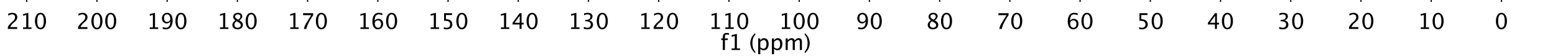


LMC-04-187-aldol.1.fid

Parameter

Data File Name

Title

Origin

Solvent

Temperature

Pulse Sequence

Number of Scans

Receiver Gain

Relaxation Delay

Pulse Width

Acquisition Time

Acquisition Date

Spectrometer Freq

Spectral Width

Lowest Frequency

Nucleus

Acquired Size

Spectral Size

/ Volumes/ nmrdata/ Ichapman/ nmr/ LMC-04-187-aldol/ 1/ fid

LMC-04-187-aldol.1.fid

Bruker BioSpin GmbH

$\mathrm{CDCl} 3$

294.9

zg30

1.0000

11.7000

4.0894

2016-05-30T20:05:30

400.13

8012.8

$-1545.6$

$1 \mathrm{H}$

32768

65536
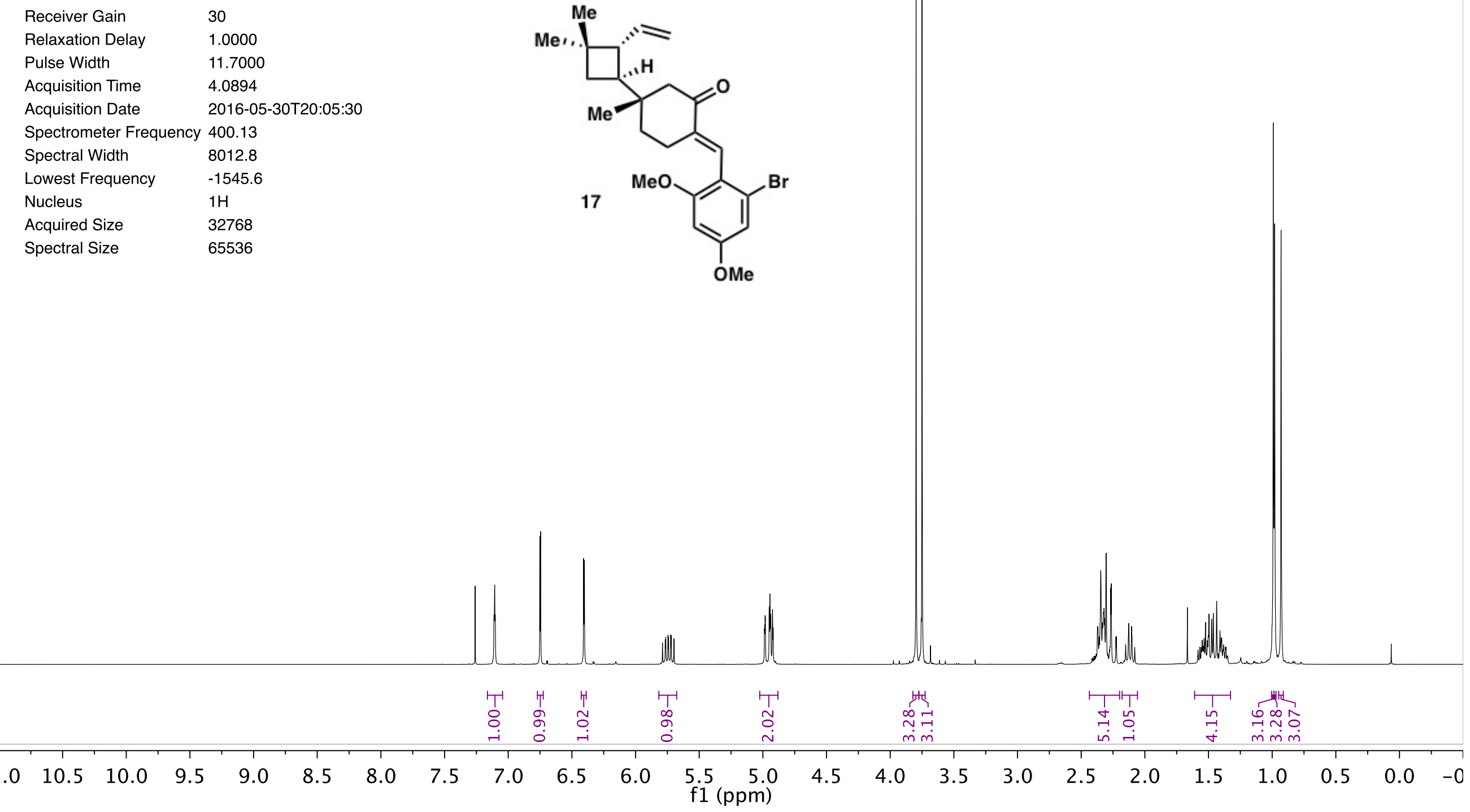


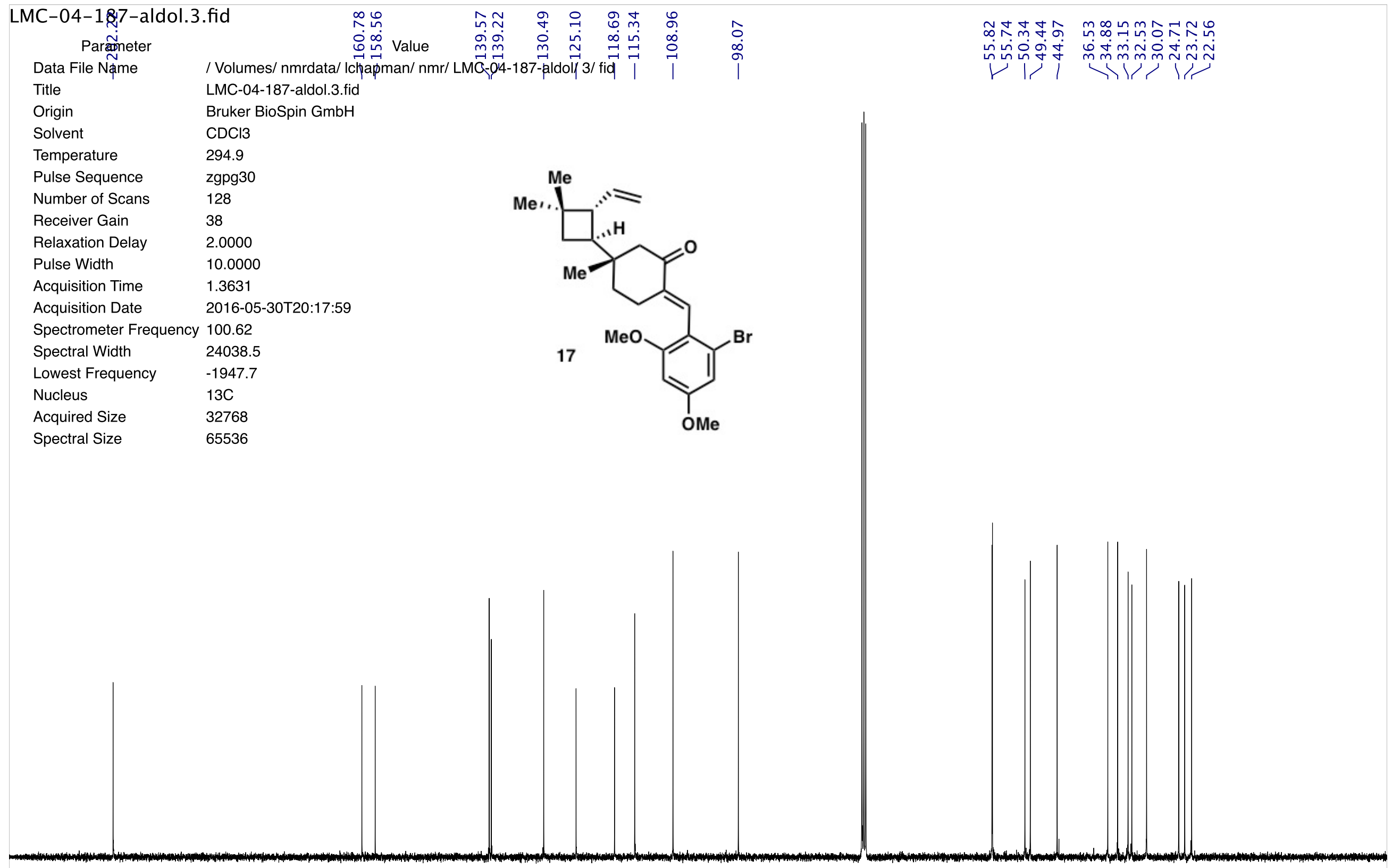

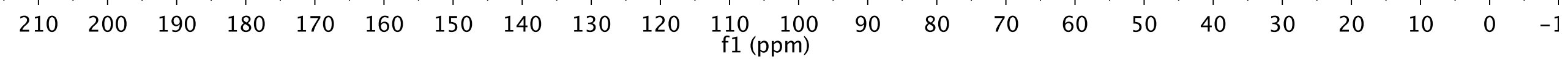


LMC-04-191-P2.1.fid

Parameter

Data File Name

/Volumes/ nmrdata/ Ichapman/ nmr/ LMC-04-191-P2/ 1/ fid

Title

MC-04-191-P2.1.fid

Origin Bruker BioSpin $\mathrm{GmbH}$

Solvent $\quad \mathrm{CDCl} 3$

Temperature 295.0

Pulse Sequence $\quad$ zg30

Number of Scans 1

Receiver Gain $\quad 30$

Relaxation Delay $\quad 1.0000$

Pulse Width $\quad 11.7000$

Acquisition Time $\quad 4.0894$

Acquisition Date 2016-06-01T06:57:13

Spectrometer Frequency 400.13

Spectral Width $\quad 8012.8$

Lowest Frequency $\quad-1545.6$

Nucleus $\quad 1 \mathrm{H}$

Acquired Size $\quad 32768$

Spectral Size $\quad 65536$
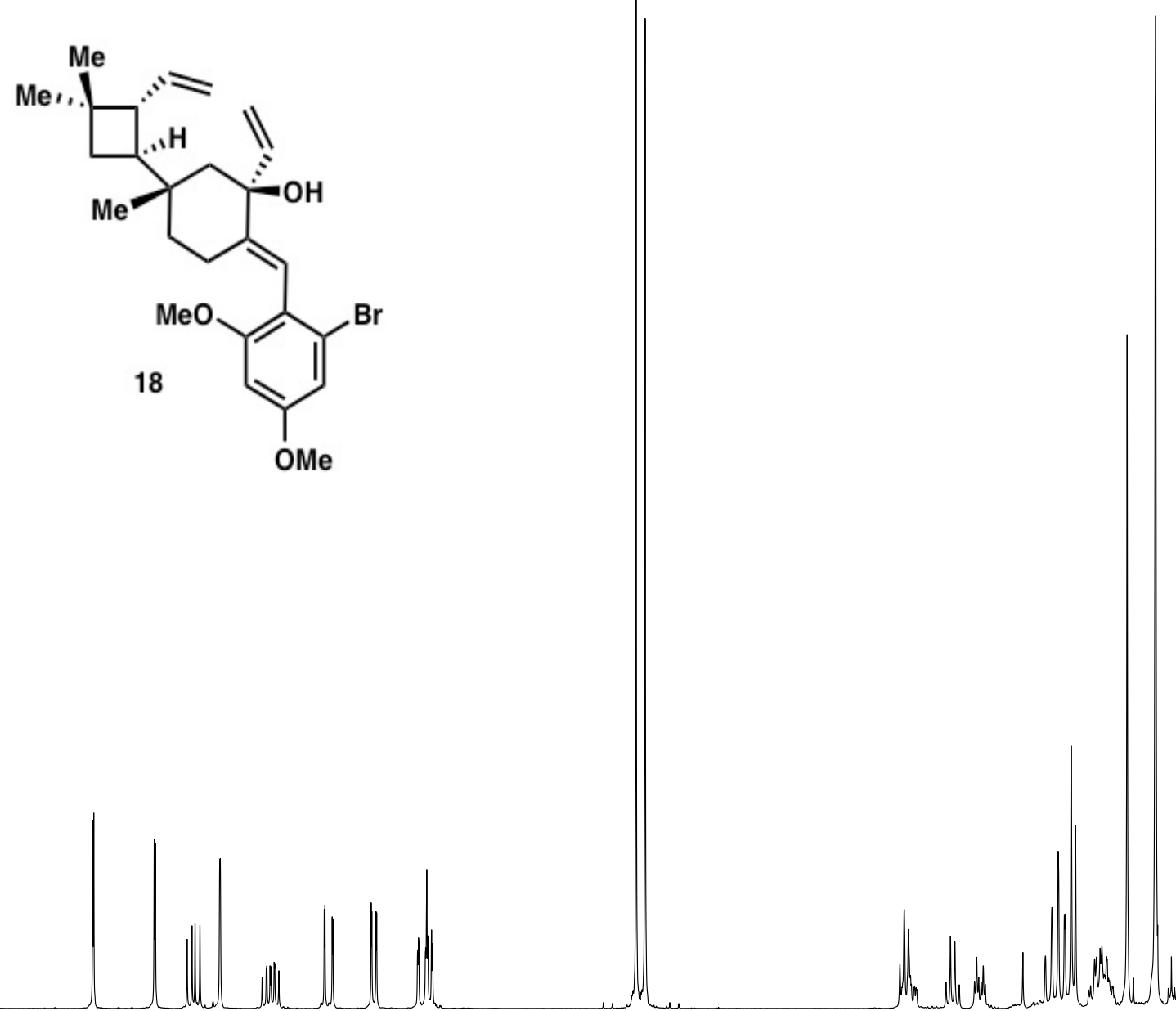

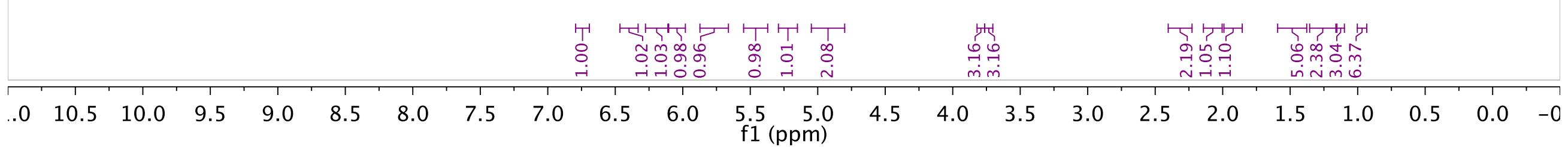




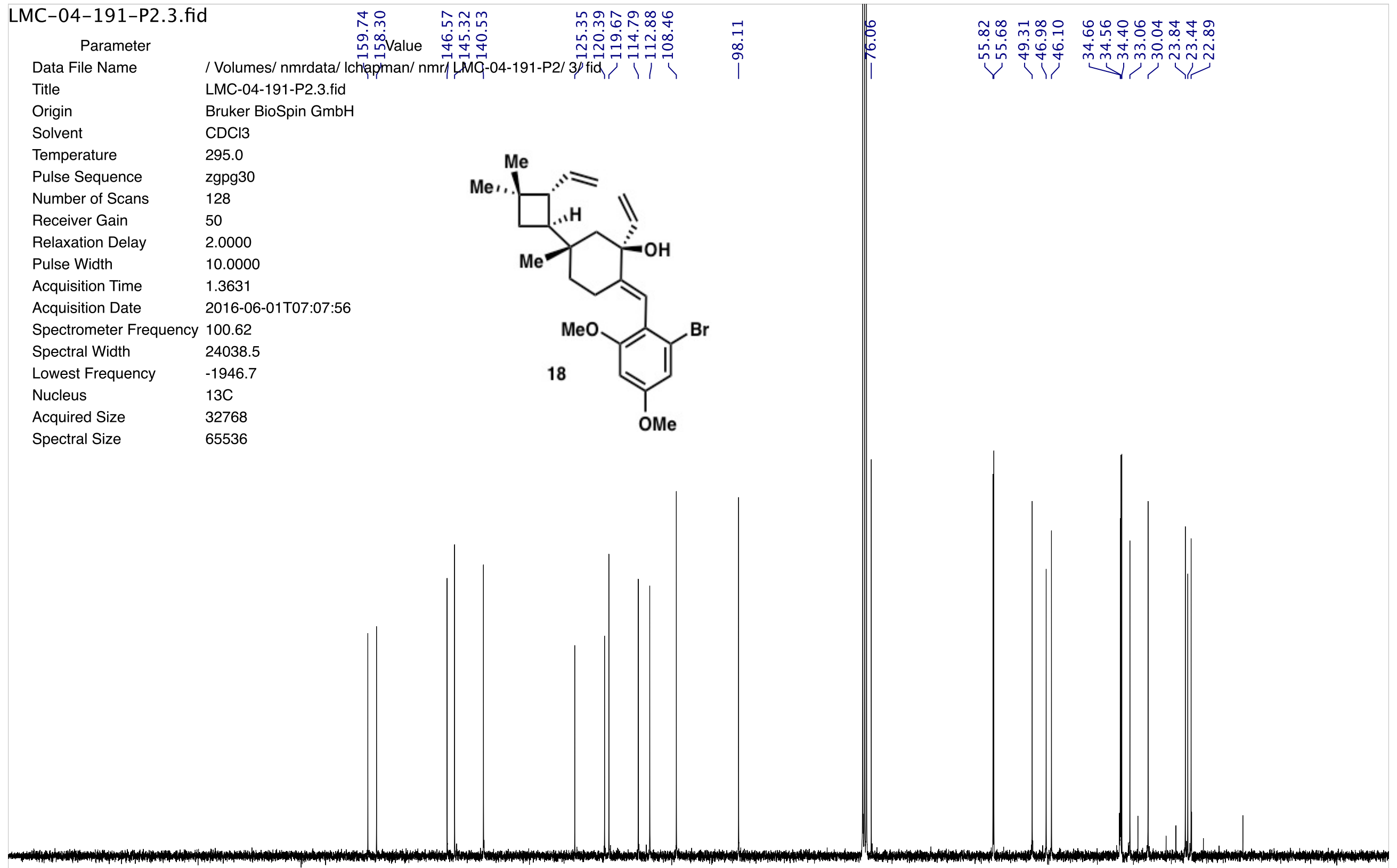

$\begin{array}{lllllllllllllllllllll}210 & 200 & 190 & 180 & 170 & 160 & 150 & 140 & 130 & 120 & \begin{array}{c}110 \\ \mathrm{f} 1(\mathrm{ppm})\end{array} & 90 & 80 & 70 & 60 & 50 & 40 & 30 & 20 & 10 & 0\end{array}$


LMC-04-191-P1.1.fid

Parameter

\section{Value}

Data File Name / Volumes/ nmrdata/ Ichapman/ nmr/ LMC-04-191-P1/ 1/ fid

Title LMC-04-191-P1.1.fid

Origin Bruker BioSpin $\mathrm{GmbH}$

Solvent $\quad \mathrm{CDCl} 3$

Temperature 294.9

Pulse Sequence $\quad \mathrm{zg} 30$

Number of Scans 1

Receiver Gain $\quad 30$

Relaxation Delay $\quad 1.0000$

Pulse Width $\quad 11.7000$

Acquisition Time $\quad 4.0894$

Acquisition Date $\quad$ 2016-06-01T15:46:17

Spectrometer Frequency 400.13

Spectral Width $\quad 8012.8$

Lowest Frequency $\quad-1545.6$

Nucleus $\quad 1 \mathrm{H}$

Acquired Size $\quad 32768$

Spectral Size $\quad 65536$
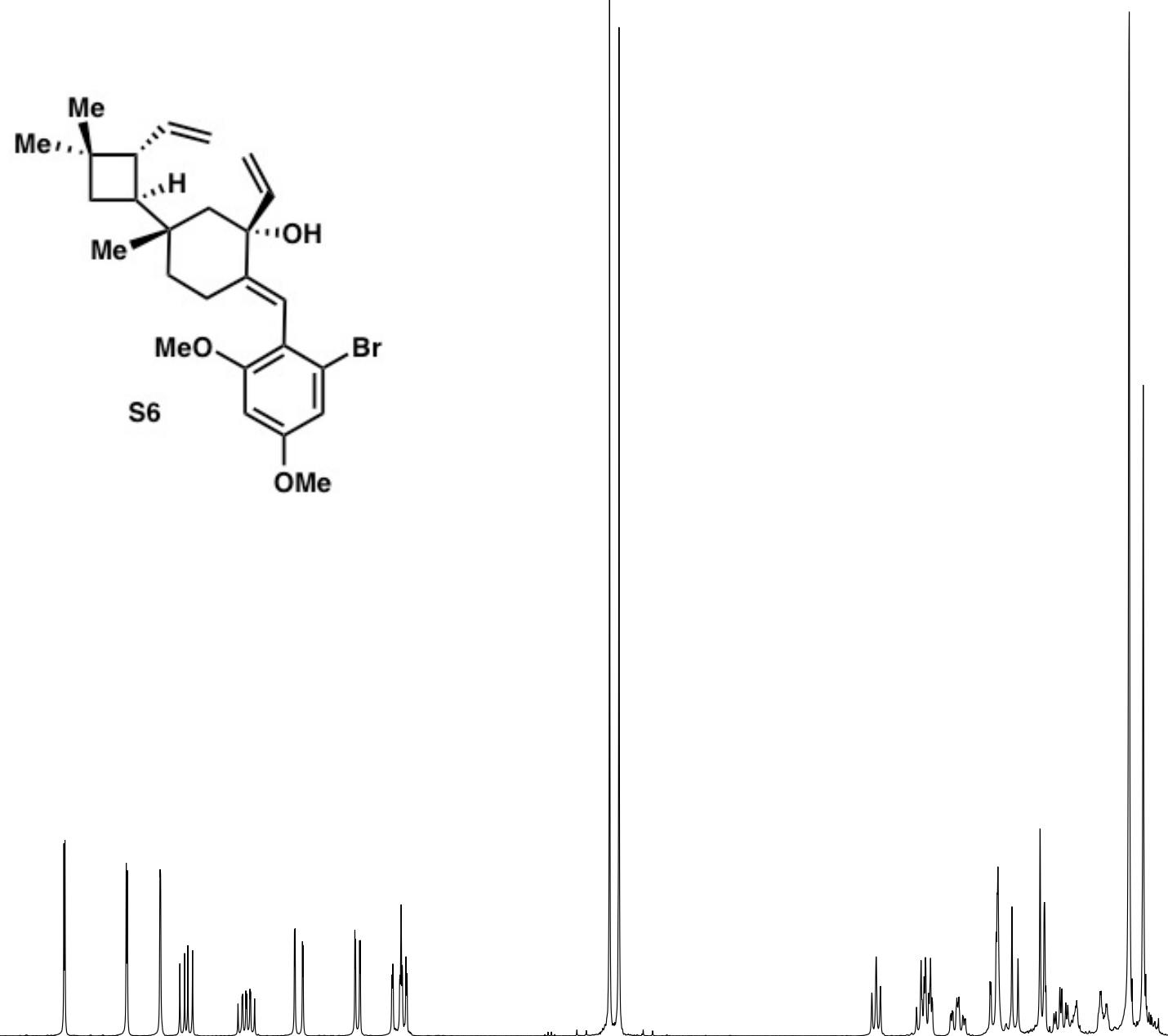

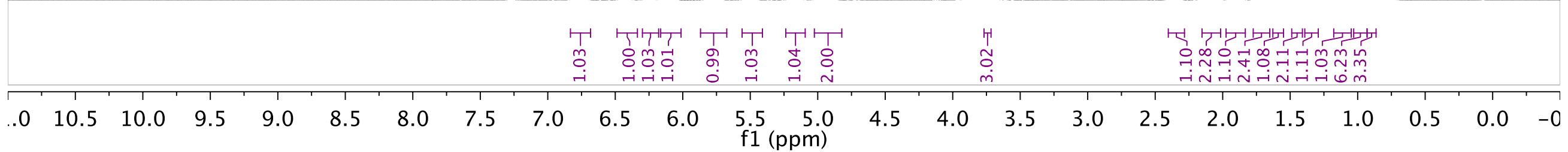




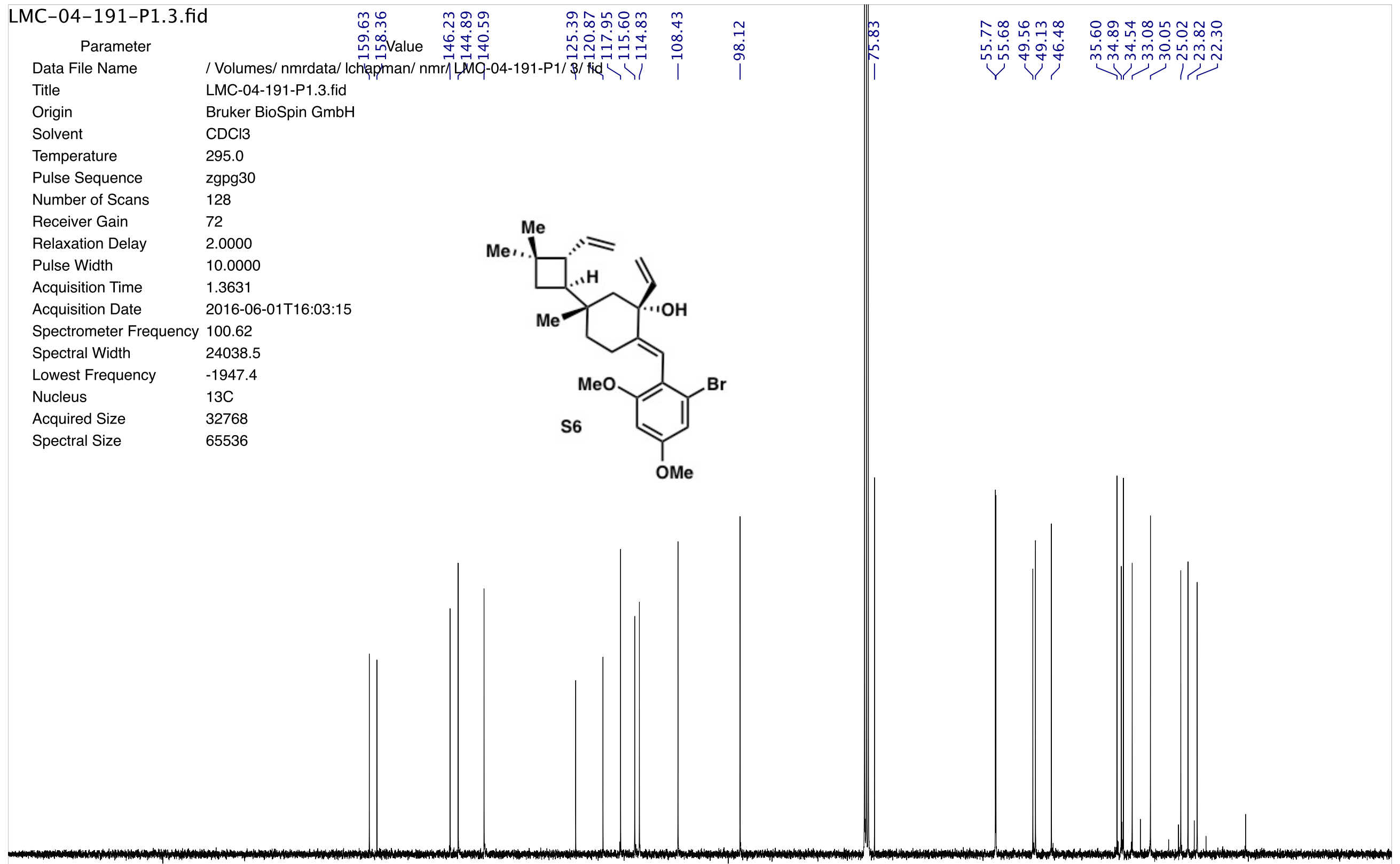

$\begin{array}{llllllllllllllllllllll}210 & 200 & 190 & 180 & 170 & 160 & 150 & 140 & 130 & 120 & \begin{array}{c}110 \\ \mathrm{f} 1(\mathrm{ppm})\end{array} & 90 & 80 & 70 & 60 & 50 & 40 & 30 & 20 & 10 & 0 & -1\end{array}$


LMC-04-192-RCM.1.fid

Parameter

Data File Name

Title

Origin

Solvent

Temperature

Pulse Sequence

Number of Scans

Receiver Gain

Relaxation Delay

Pulse Width

Acquisition Time

Acquisition Date

Spectrometer Fr

Spectral Width

Lowest Frequency

Nucleus

Acquired Size

Spectral Size

Value
/ Volumes/ nmrdata/ Ichapman/ nmr/ LMC-04-192-RCM/ 1/ fid

LMC-04-192-RCM.1.fid

Bruker BioSpin GmbH

$\mathrm{CDCl} 3$

295.0

zg30

1.0000

11.7000

4.0894

2016-06-02T11:26:56

400.13

8012.8

$-1545.6$

$1 \mathrm{H}$

32768

65536
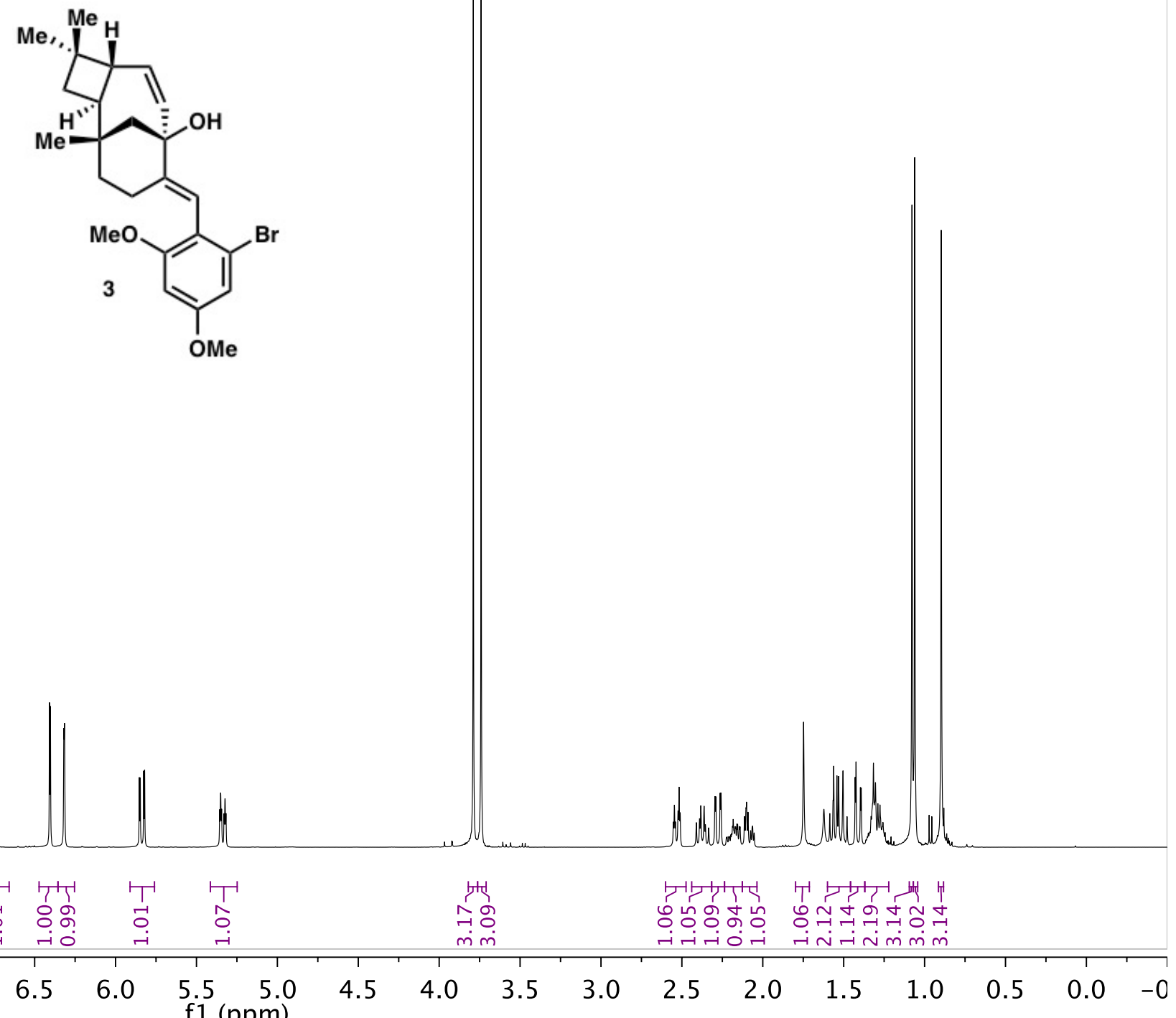

$\begin{array}{llll}0 & 10.5 & 10.0\end{array}$

$9.5 \quad 9.0$

8.5

$\begin{array}{lll}8.0 & 7.5 & 7.0\end{array}$

6.5

$6.0 \quad 5.5 \quad 5.0$

f1 (ppm)

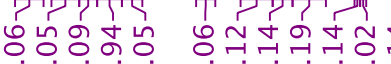

-iंir rintrimm 


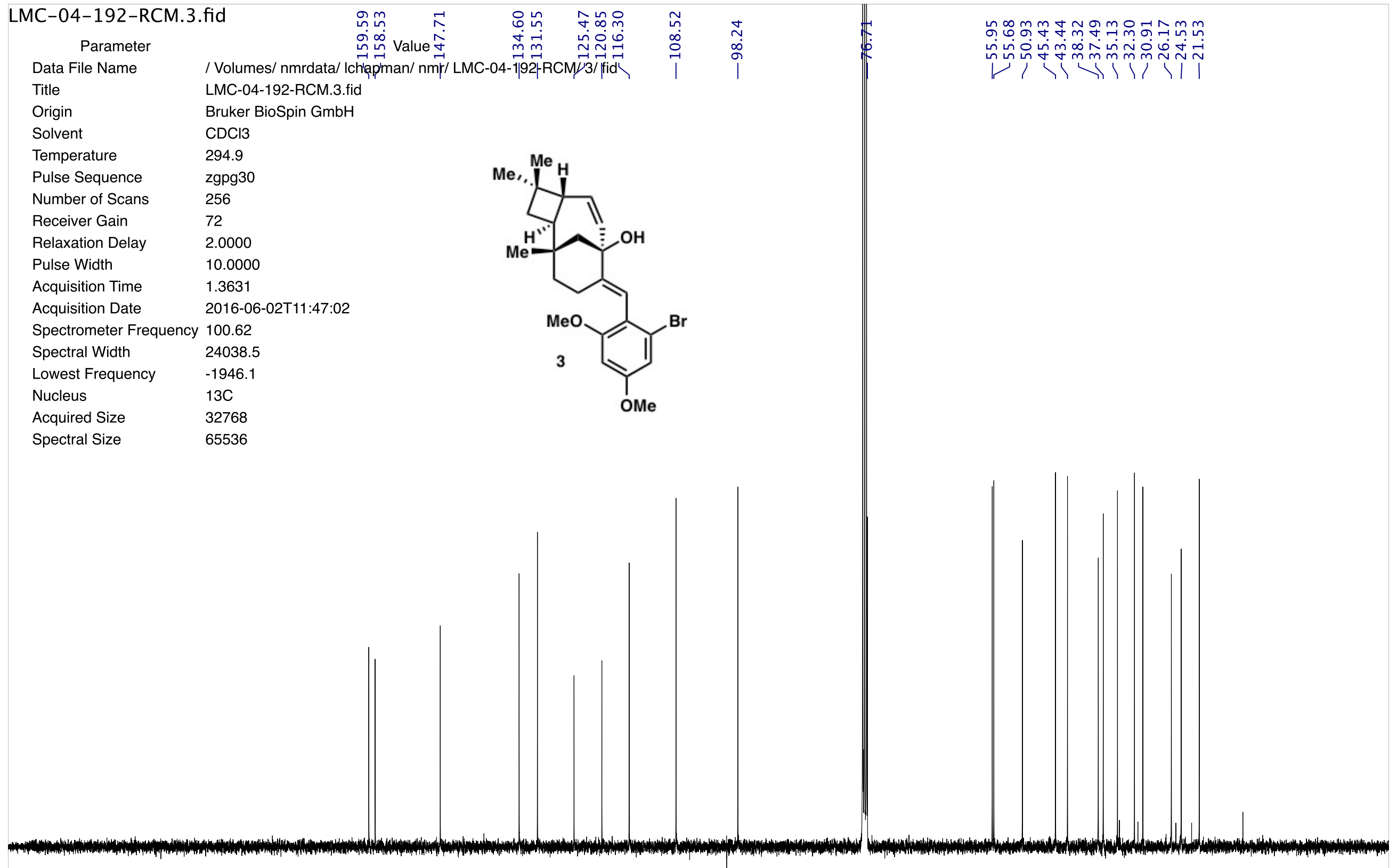

$\begin{array}{lllllllllllllllllllllll}210 & 200 & 190 & 180 & 170 & 160 & 150 & 140 & 130 & 120 & \underset{f 1}{110} \begin{array}{l}100 \\ (\mathrm{ppm})\end{array} & 90 & 80 & 70 & 60 & 50 & 40 & 30 & 20 & 10 & 0 & -1\end{array}$


LMC-04-193-hydrog.4.fid

Parameter

Data File Name

Title

Origin

Solvent

Temperature

Pulse Sequence

Number of Scans

Receiver Gain

Relaxation Delay

Pulse Width

Acquisition Time

Acquisition Date

Spectrometer Fr

Spectral Width

Lowest Frequency

Nucleus

Acquired Size

Spectral Size

\section{Value}

/ Volumes/ nmrdata/ Ichapman/ nmr/ LMC-04-193-hydrog/ 4/ fid

LMC-04-193-hydrog.4.fid

Bruker BioSpin GmbH

$\mathrm{CDCl} 3$

295.0

zg30

16

10.0000

11.7000

4.0894

2016-06-03T11:09:04

400.13

8012.8

$-1545.6$

$1 \mathrm{H}$

32768

65536
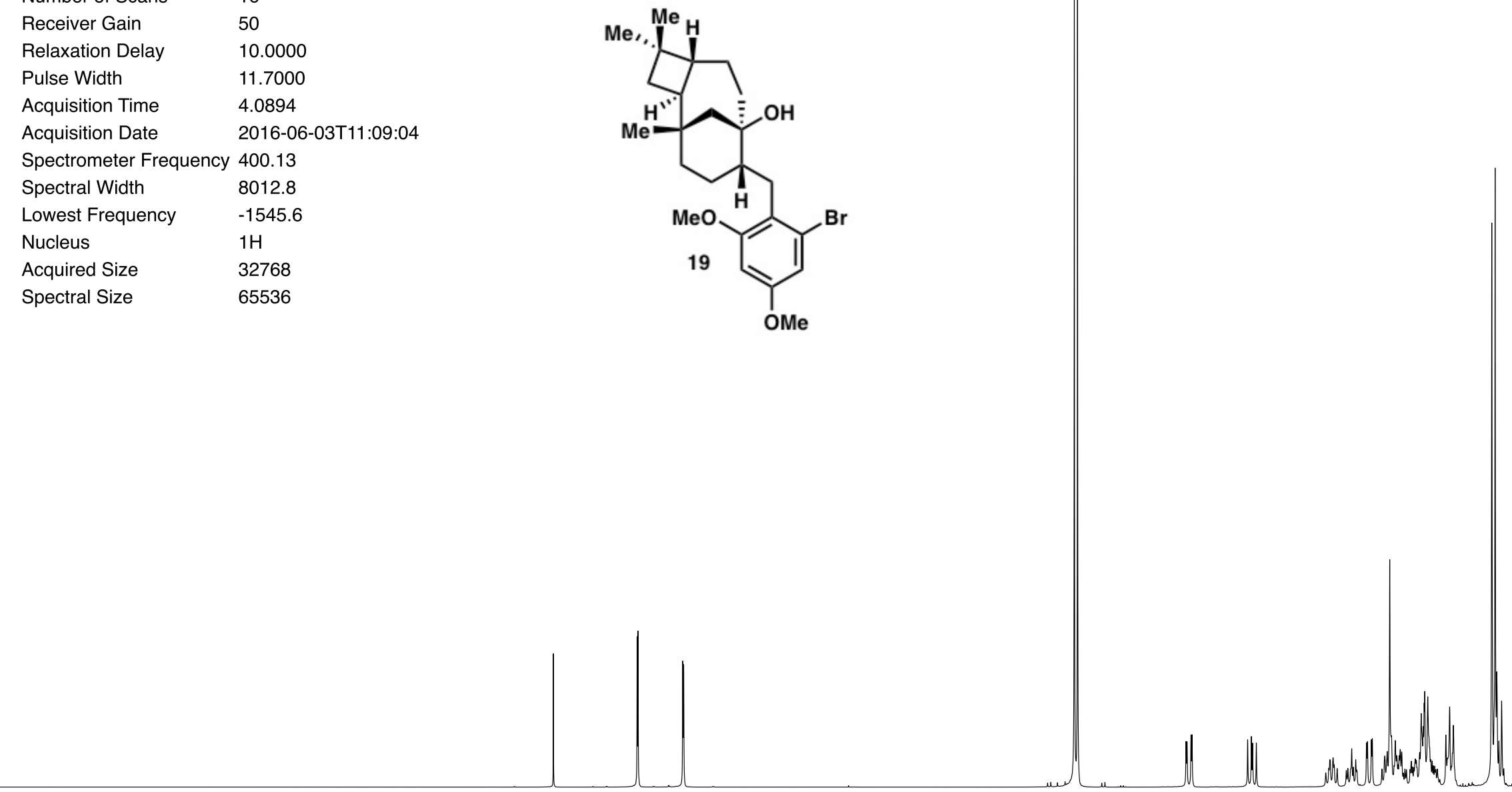

\begin{tabular}{|c|c|c|c|c|c|c|c|c|c|c|c|c|c|c|c|c|c|c|c|c|c|c|c|}
\hline & & & & & & & & & $\begin{array}{ll}T & 1 \\
1 & 0 \\
0 & 8 \\
- & - \\
-1 & -1\end{array}$ & & & & & \begin{tabular}{l}
$f$ \\
\multirow{2}{0}{} \\
$\dot{m}$
\end{tabular} & & $\begin{array}{l}\text { 'T } \\
\text { बे } \\
0 \\
0\end{array}$ & $\begin{array}{l}\text { †' } \\
\text { ळे } \\
0 \\
0\end{array}$ & 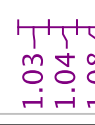 & 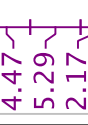 & 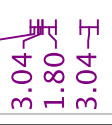 & & & \\
\hline .0 & 10.5 & 10.0 & 9.5 & 9.0 & 8.5 & 8.0 & 7.5 & 7.0 & 6.5 & 6.0 & $\begin{array}{c}5.5 \\
\mathrm{f} 1\end{array}$ & $\begin{array}{l}5.0 \\
\text { om) }\end{array}$ & 4.5 & 4.0 & 3.5 & 3.0 & 2.5 & 2.0 & 1.5 & 1.0 & 0.5 & 0.0 & -0 \\
\hline
\end{tabular}




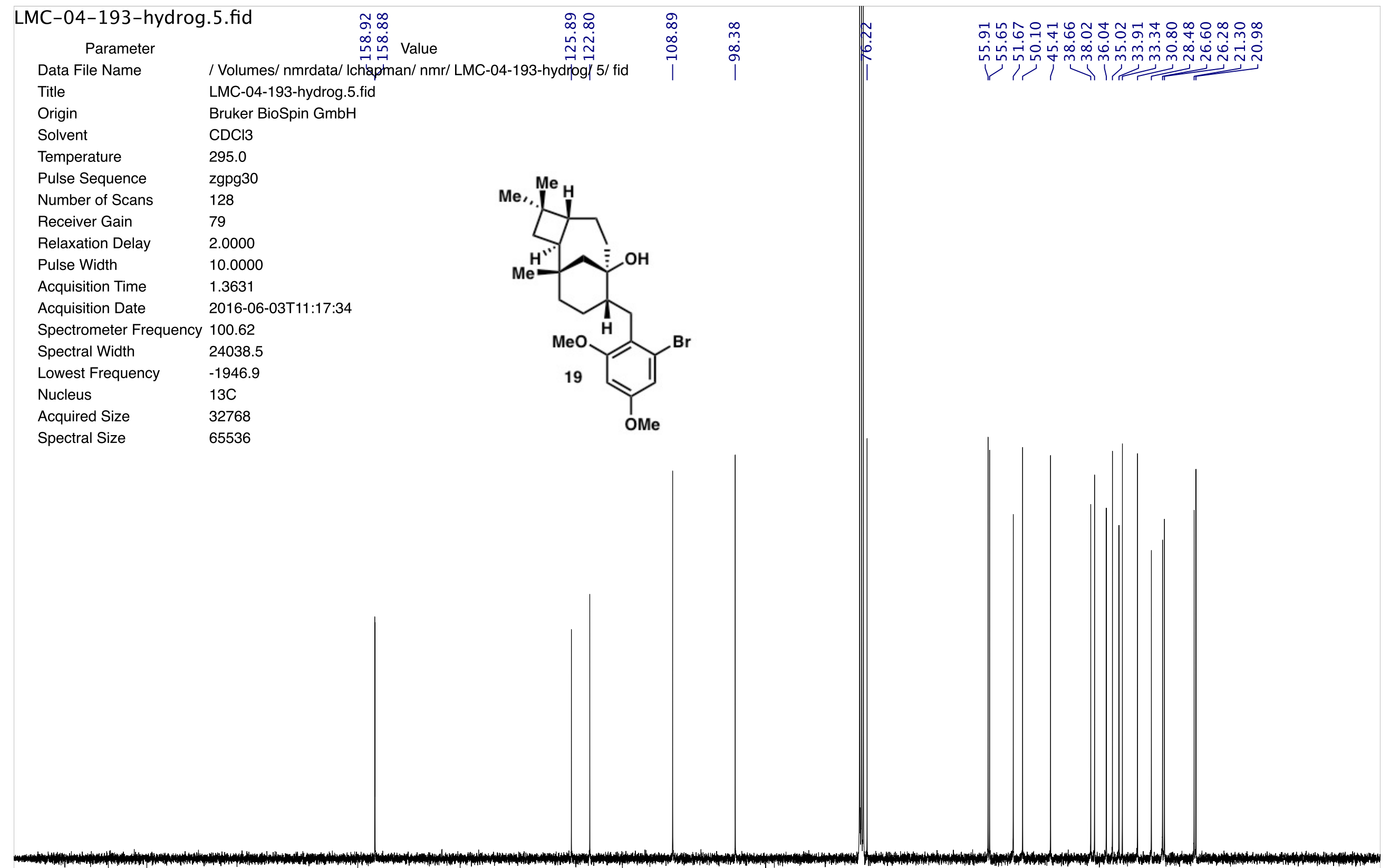

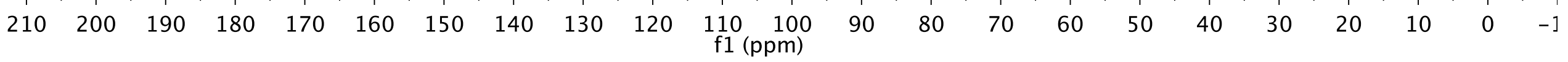


LMC-04-193-P1.2.fid

Parameter

Data File Name

Title

Origin

Solvent

Temperature

Pulse Sequence

Number of Scans

Receiver Gain

Relaxation Delay

Pulse Width

Acquisition Time

Acquisition Date

Spectrometer Freq

Spectral Width

Lowest Frequency

Nucleus

Acquired Size

Spectral Size
Volumes/ nmrdata/ Ichapman/ nmr/ LMC-04-193-P1/ 2/ fid

LMC-04-193-P1.2.fid

Bruker BioSpin GmbH

$\mathrm{CDCl} 3$

295.0

zg30

16

30

10.0000

11.7000

4.0894

2016-06-04T01:48:10

400.13

8012.8

$-1545.6$

$1 \mathrm{H}$

32768

65536
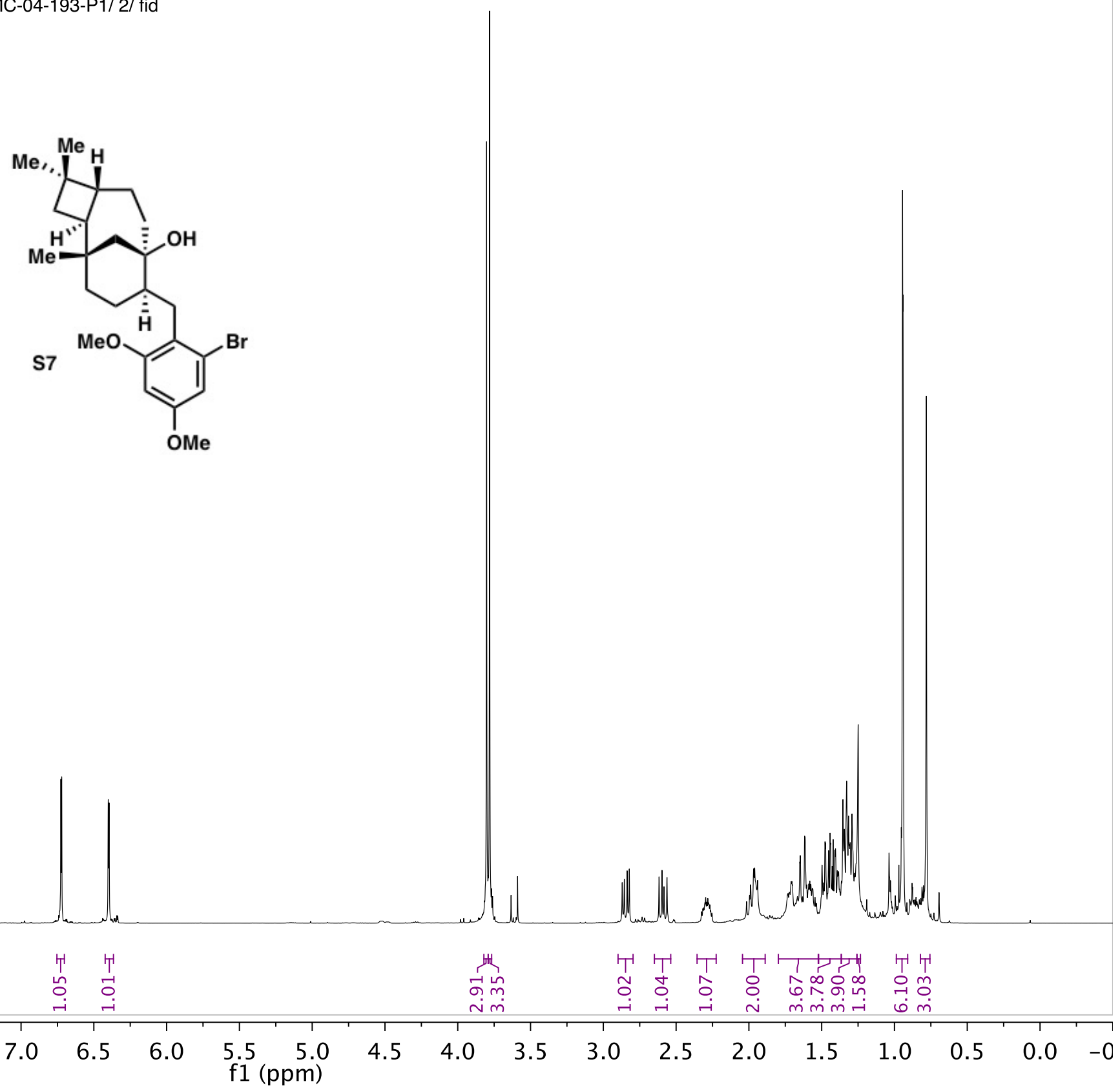

9.59 .0

8.5

$\begin{array}{ll}7.5 & 7.0\end{array}$

f1 (ppm) 


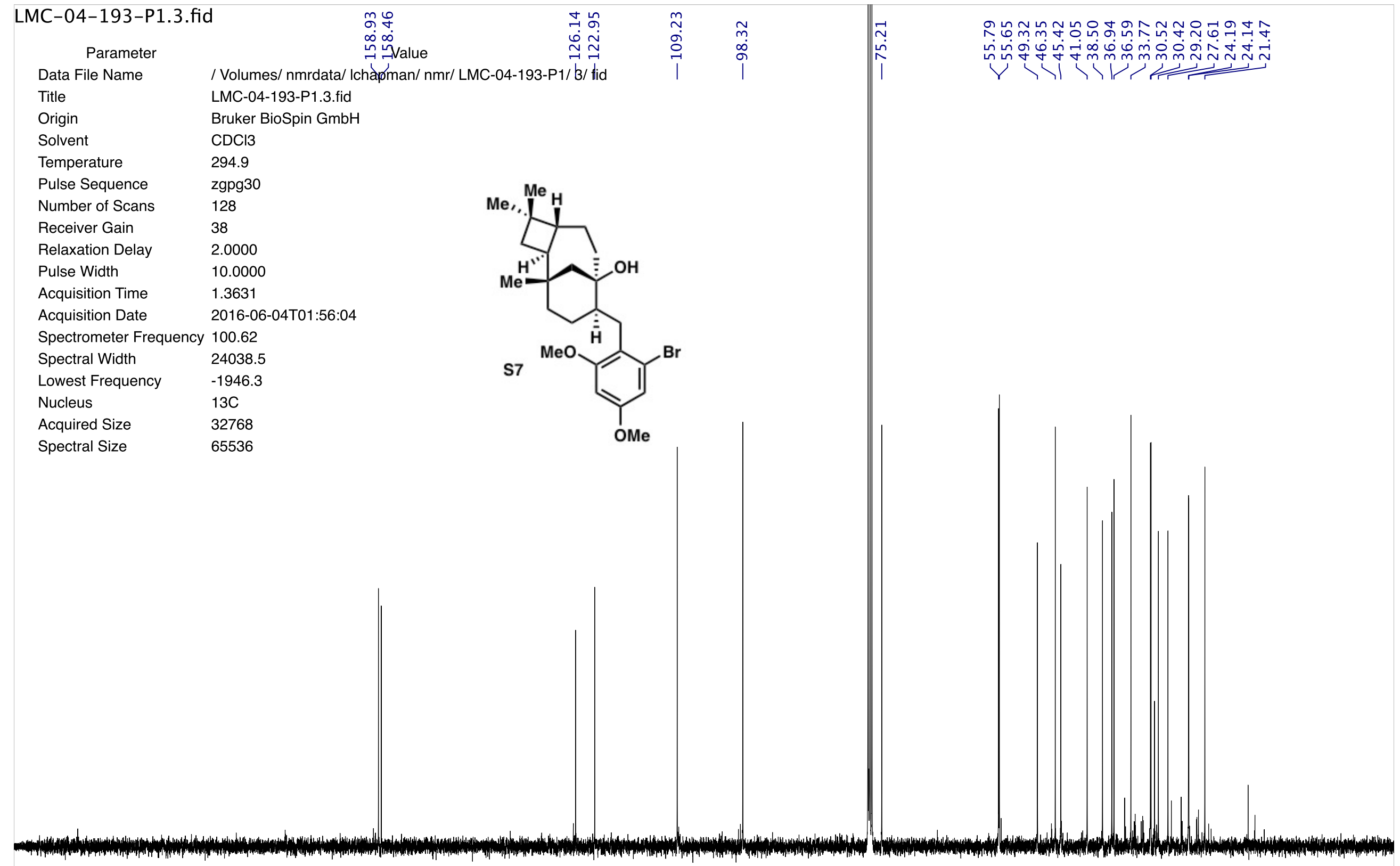

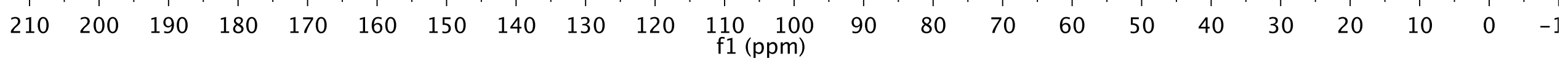


LMC-04-194-pentacy.1.fid

Parameter

Data File Name

Title

Origin

Solvent

Temperature

Pulse Sequence

Number of Scans

Receiver Gain

Relaxation Delay

Pulse Width

Acquisition Time

Acquisition Date

Spectrometer Frequ

Spectral Width

Lowest Frequency

Nucleus

Acquired Size

Spectral Size

MC-04-194-pentacy.1.fid

Bruker BioSpin GmbH

$\mathrm{CDCl} 3$

294.9

zg30

1.0000

11.7000

4.0894

2016-06-04T02:00:16

400.13

8012.8

$-1545.6$

$1 \mathrm{H}$

32768

65536

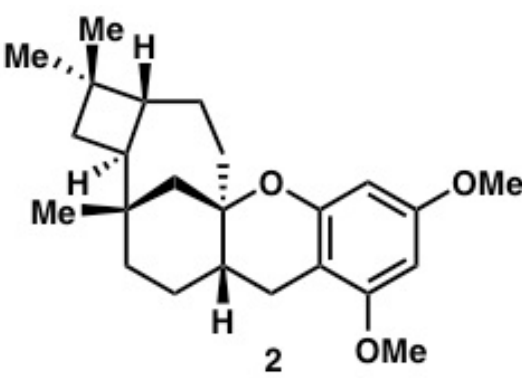

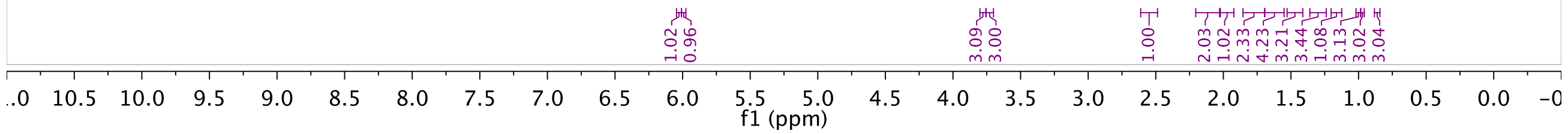




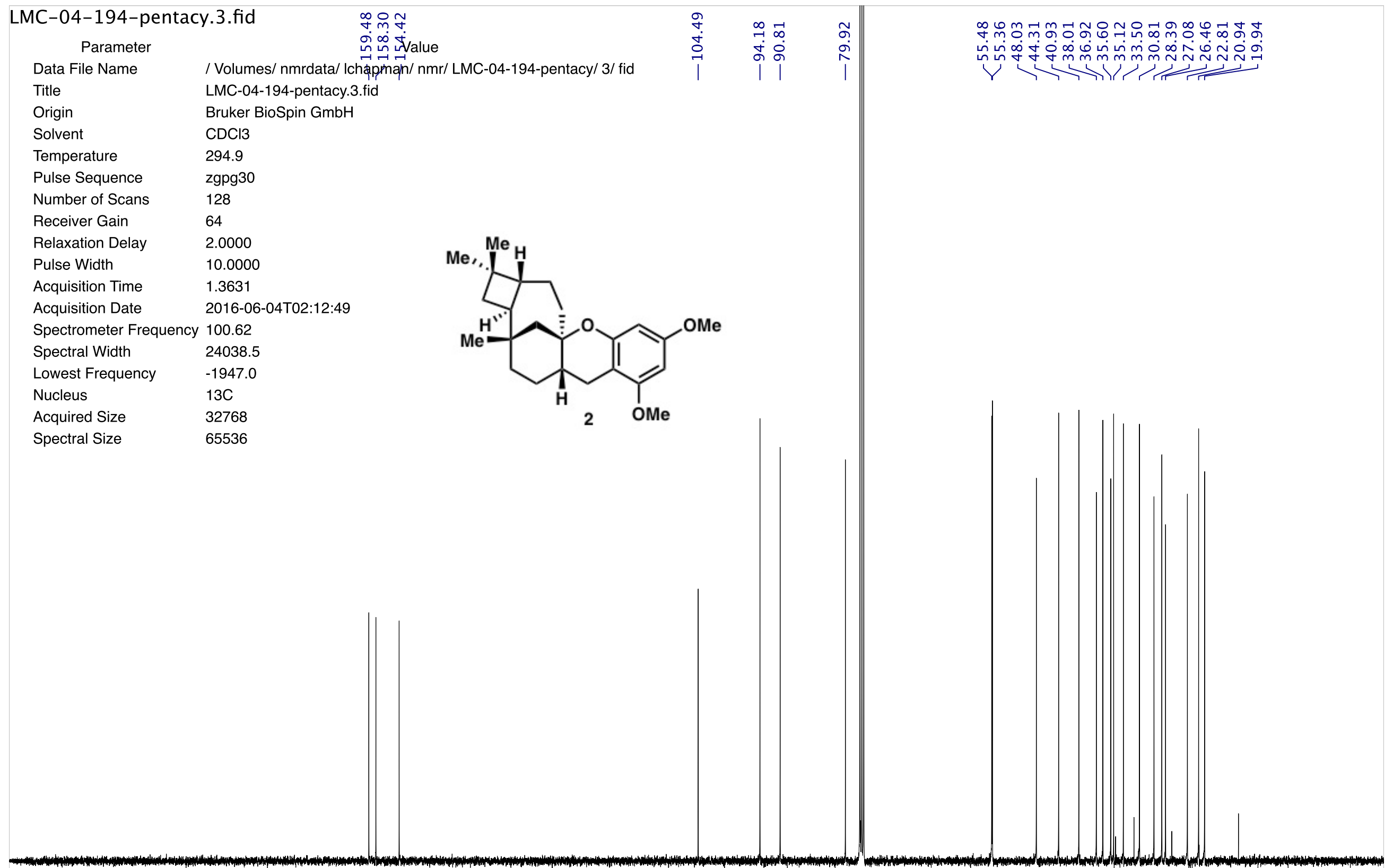

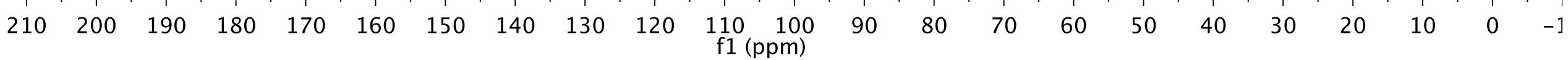


LMC-04-199-pTLC-1.4.fid

Parameter

Data File Name

Title

Origin

Solvent

Temperature

Pulse Sequence

Number of Scans

Receiver Gain

Relaxation Delay

Pulse Width

Acquisition Time

Acquisition Date

Spectrometer Frequ

Spectral Width

Lowest Frequency

Nucleus

Acquired Size

Spectral Size

Value

(199-pTLC-1/ 4/ fid

MC-04-199-pTLC-1.4 fid

Bruker BioSpin GmbH

$\mathrm{CDCl} 3$

294.9

zg30

1.0000

11.7000

4.0894

2016-06-08T21:11:24

400.13

8012.8

$-1545.6$

$1 \mathrm{H}$

32768

65536
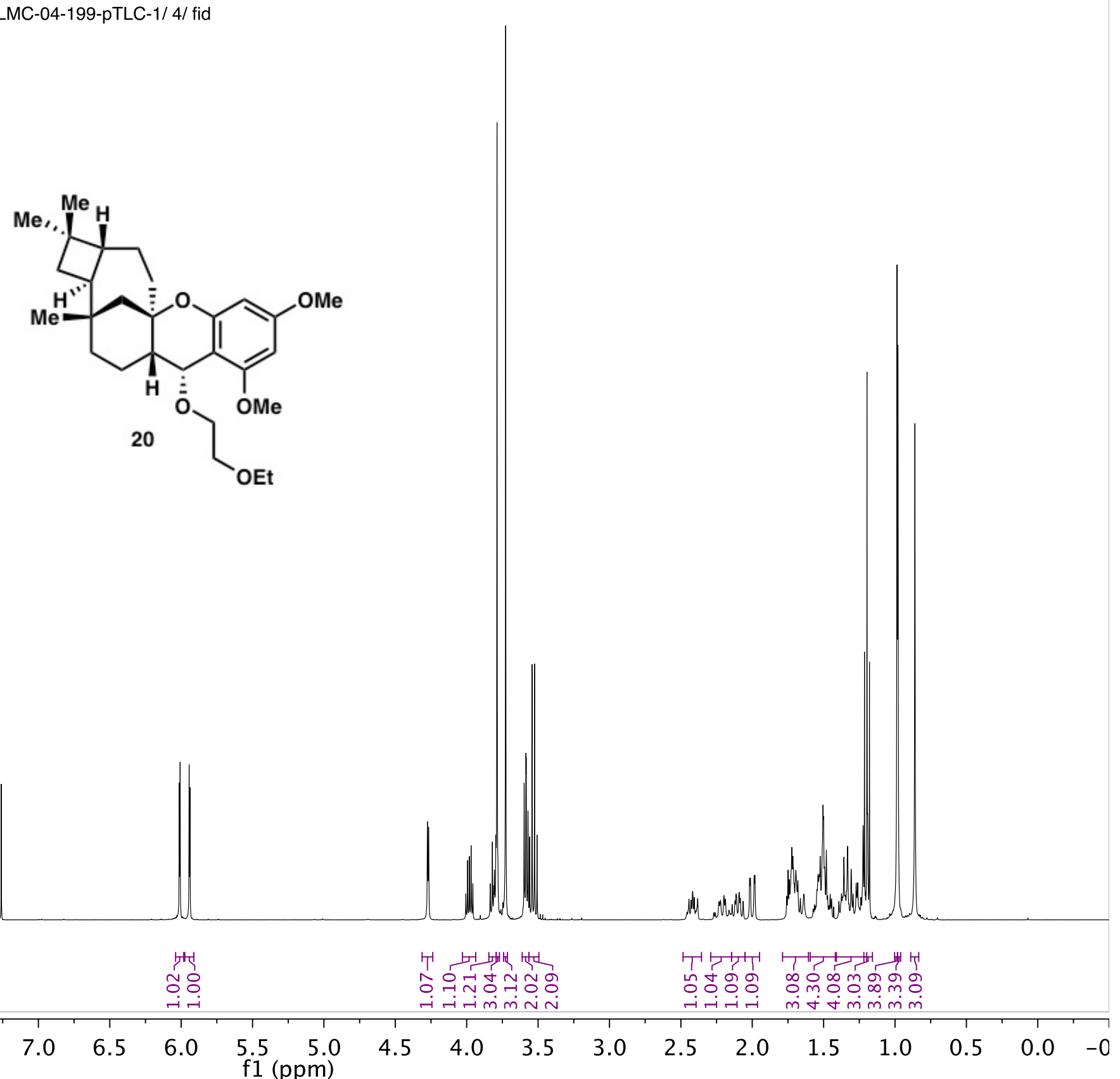

$9.5 \quad 9.0$

8.5

8.0

$\begin{array}{ll}7.5 & 7.0\end{array}$

$\begin{array}{llr}6.0 & 5.5 & 5.0\end{array}$

f1 (ppm) 


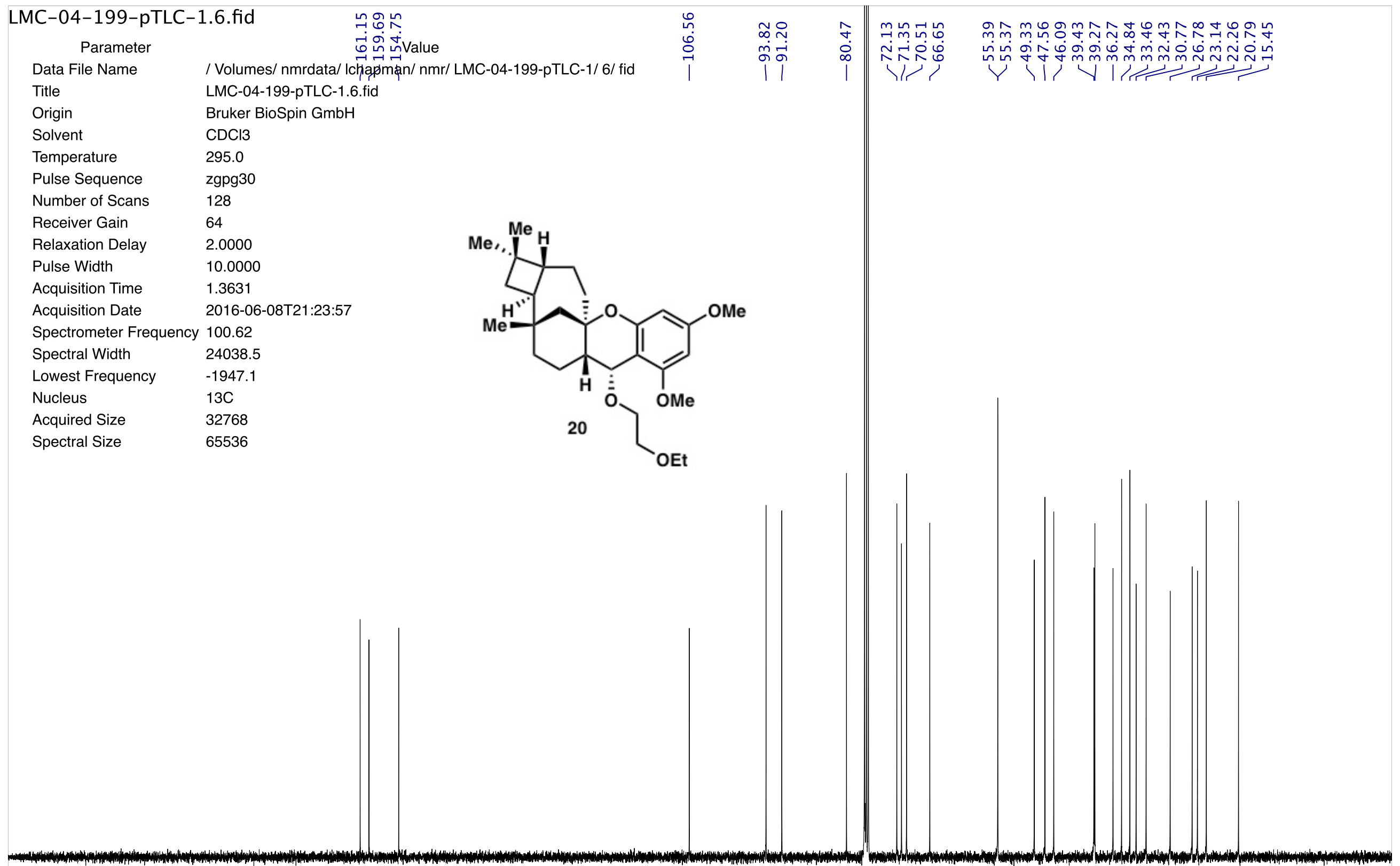

$\begin{array}{llllllllllllllllllllll}210 & 200 & 190 & 180 & 170 & 160 & 150 & 140 & 130 & 120 & \begin{array}{c}110 \\ \mathrm{f} 1(\mathrm{ppm})\end{array} & 90 & 80 & 70 & 60 & 50 & 40 & 30 & 20 & 10 & 0 & -1\end{array}$


LMC-04-199-pTLC-2.1.fid

Parameter

Data File Name

Title

Origin

Solvent

Temperature

Pulse Sequence

Number of Scans

Receiver Gain

Relaxation Delay

Pulse Width

Acquisition Time

Acquisition Date

Spectrometer Frequ

Spectral Width

Lowest Frequency

Nucleus

Acquired Size

Spectral Size

Value

(19)-pTLC-2/ 1/ fid

LMC-04-199-pTLC-2.1.fid

Bruker BioSpin GmbH

$\mathrm{CDCl} 3$

295.0

zg30

127

1.0000

11.7000

4.0894

2016-06-08T21:28:51

400.13

8012.8

$-1545.6$

$1 \mathrm{H}$

32768

65536
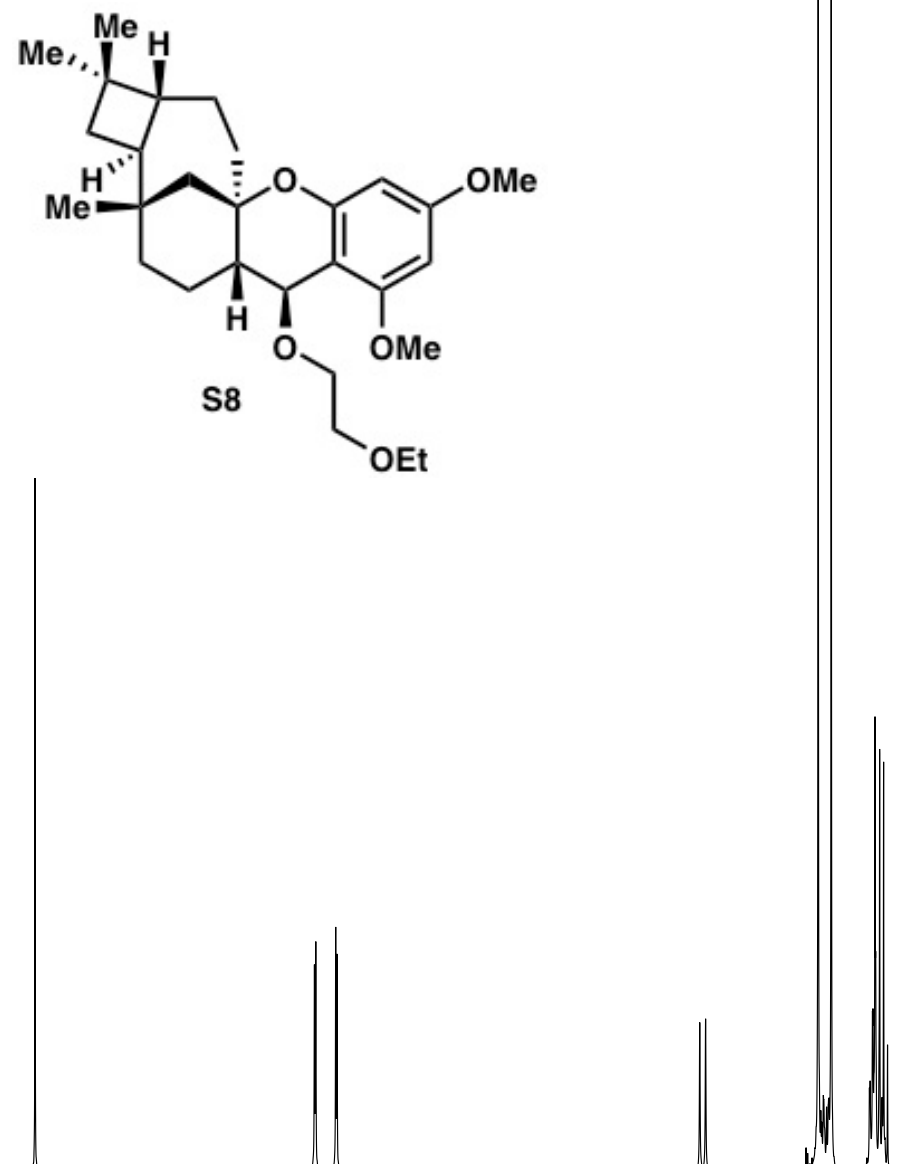

以下!十正田

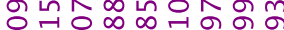

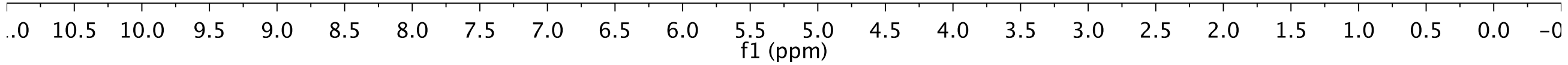




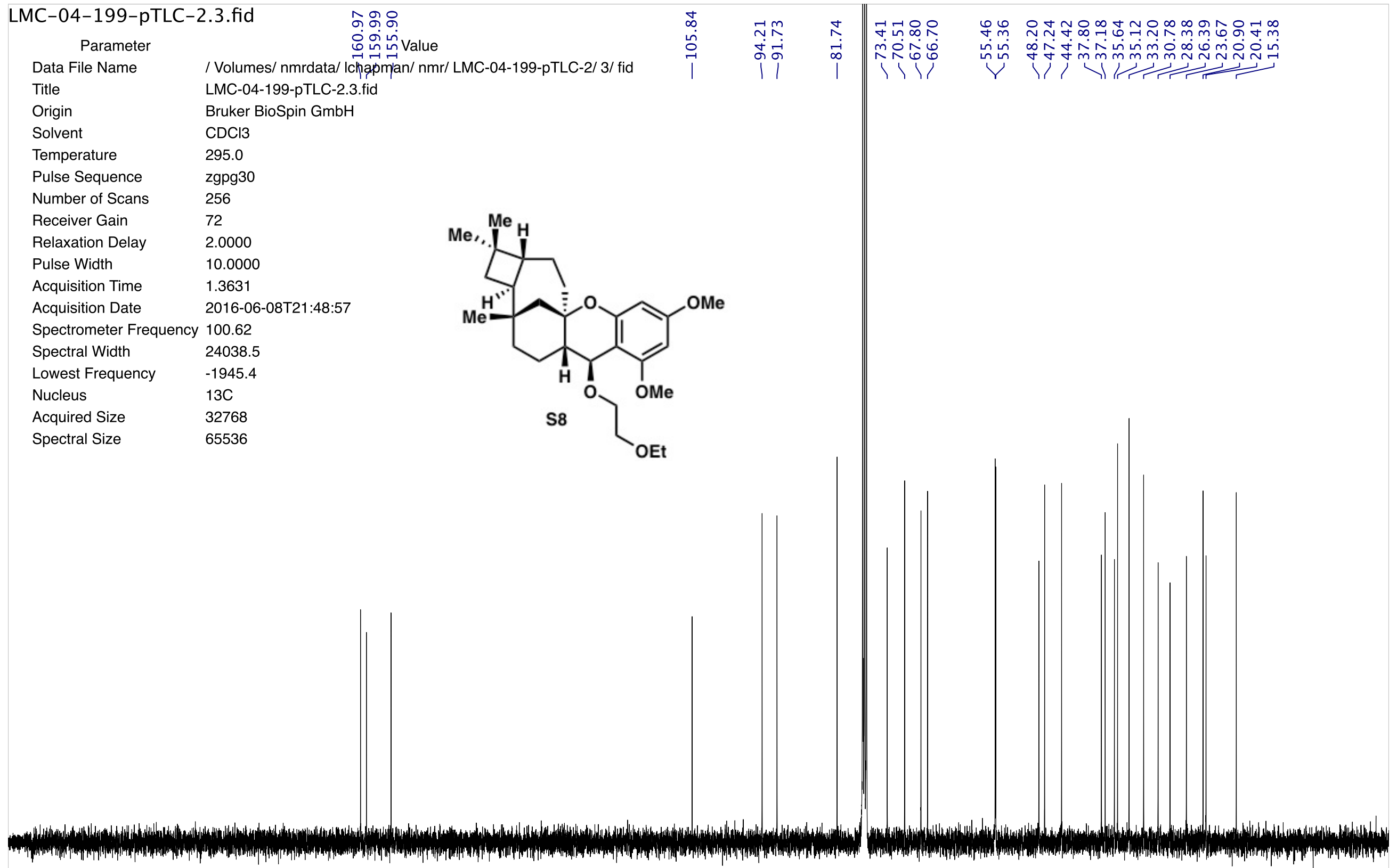

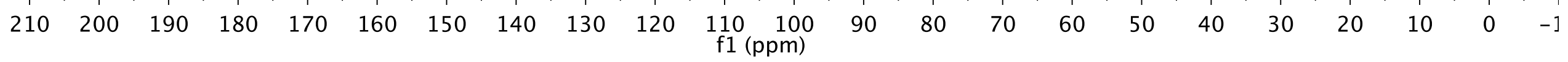




\section{PROTON01}

LMC-9a4-205-flash

Value

Data File Name / Volumes/ nmrdata-1/ Ichapman/ vnmrsys/ data/ LMC-04-205-flash/ PROTON01.fid/ fid Title PROTON01

Comment LMC-04-205-flash

Origin Varian

Spectrometer inova

Solvent cdcl3

Temperature $\quad 25.0$

Pulse Sequence s2pul

Experiment 1D

Probe autox7991

Number of Scans 1

Receiver Gain 24

Relaxation Delay 1.0000

Pulse Width

0.0000

Acquisition Time 3.0000

Acquisition Date 2016-06-11T18:39:19

Nucleus $\quad 1 \mathrm{H}$
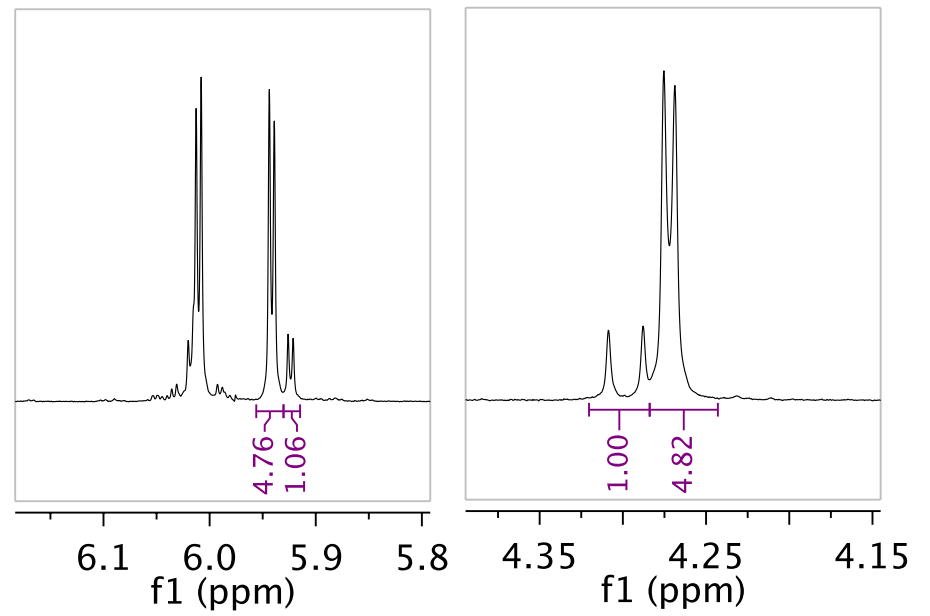

mixture as used in phenylation
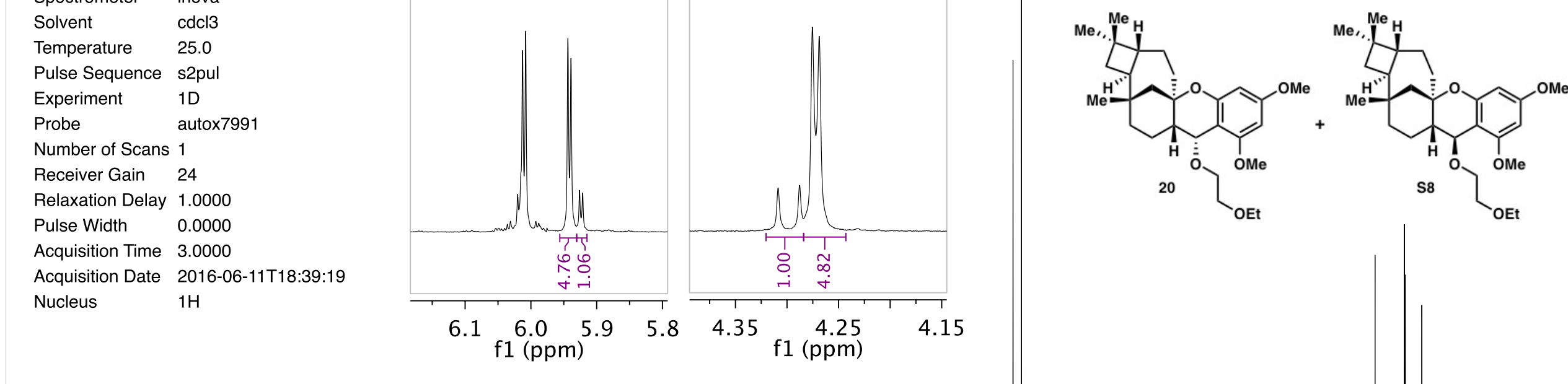

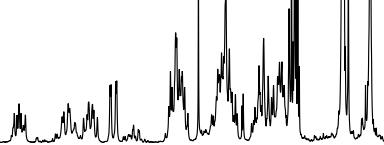

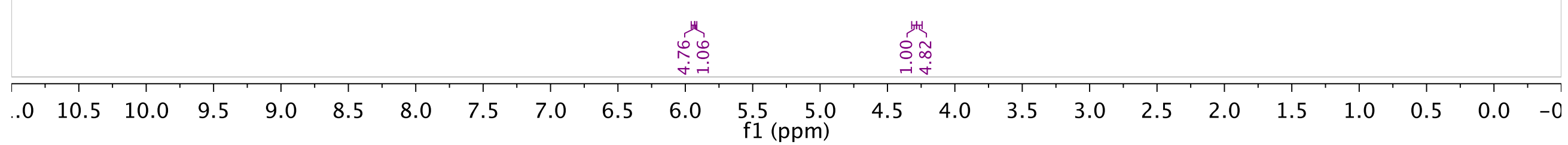




\section{CARBON01}

LMC-Patantretef-flash-c13

Data File Name

Title

Comment

Origin

Spectrometer

Solvent

Temperature

Pulse Sequence s2pul

Experiment

Probe

$$
\text { 1D }
$$

Number of Scans 1000

Receiver Gain 30

Relaxation Delay 1.0000

Pulse Width

0.0000

Acquisition Time 1.0420

Acquisition Date 2016-06-11T18:49:53

Nucleus

$13 \mathrm{C}$

Volumes/

MC-04-205-flash-c13

Varian

$0 \times 7991$

ํํํํํำ

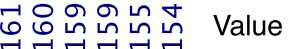

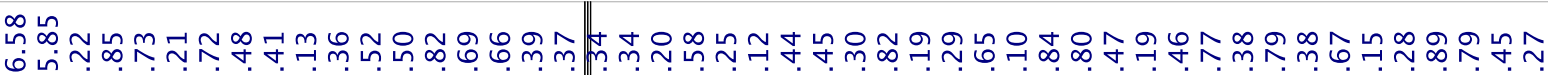

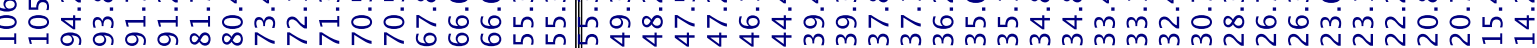
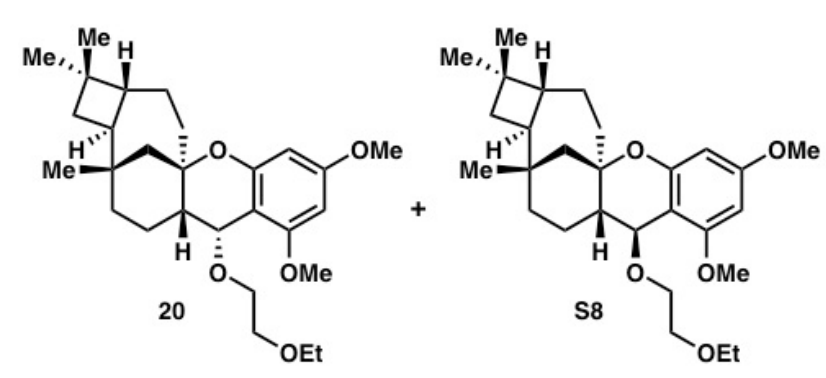

mixture as used in phenylation

OEt

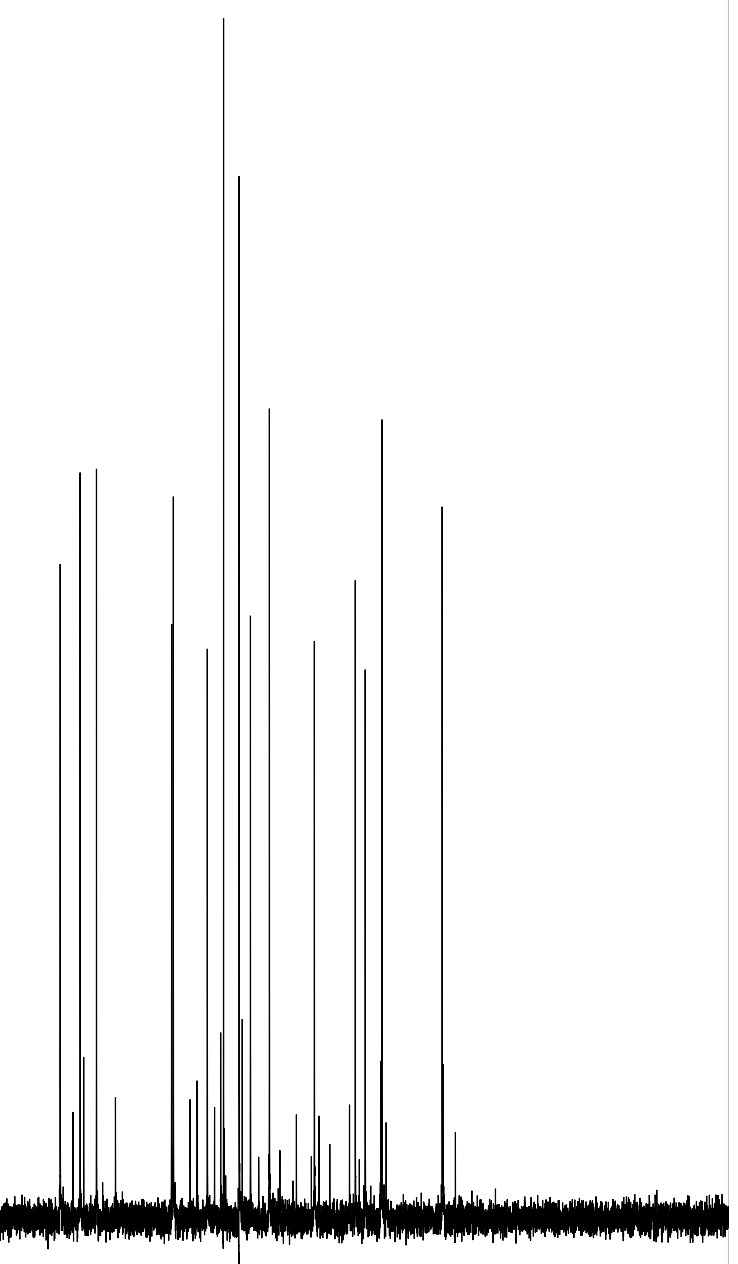

?

$20 \quad 210$

200

190

180

170

160

150

140

130

120

$110 \underset{\text { f1 }}{10 p m)}$

90

80

70

60

50

40

30

20

$\left.\begin{array}{lll}10 & 0 & -\end{array}\right]$ 
Parameter

Data File Name

Title

Origin

Solvent

Temperature

Pulse Sequence

Number of Scans

Receiver Gain

Relaxation Delay

Pulse Width

Acquisition Time

Acquisition Date

Spectrometer Fr

Spectral Width

Lowest Frequency

Nucleus

Acquired Size

Spectral Size
Value

/ Volumes/ nmrdata/ Ichapman/ nmr/ LMC-04-208/ 1/ fid

LMC-04-208.1.fid

Bruker BioSpin GmbH

$\mathrm{CDCl} 3$

mixture as used in demethylation

zg30

1.0000

11.7000

4.0894

2016-06-13T13:14:19
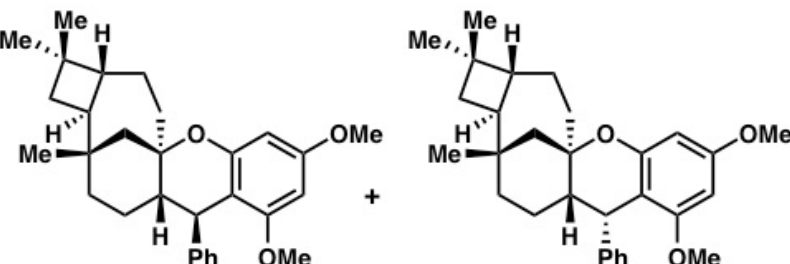

s9

8012.8

$-1545.2$

$1 \mathrm{H}$

32768

65536

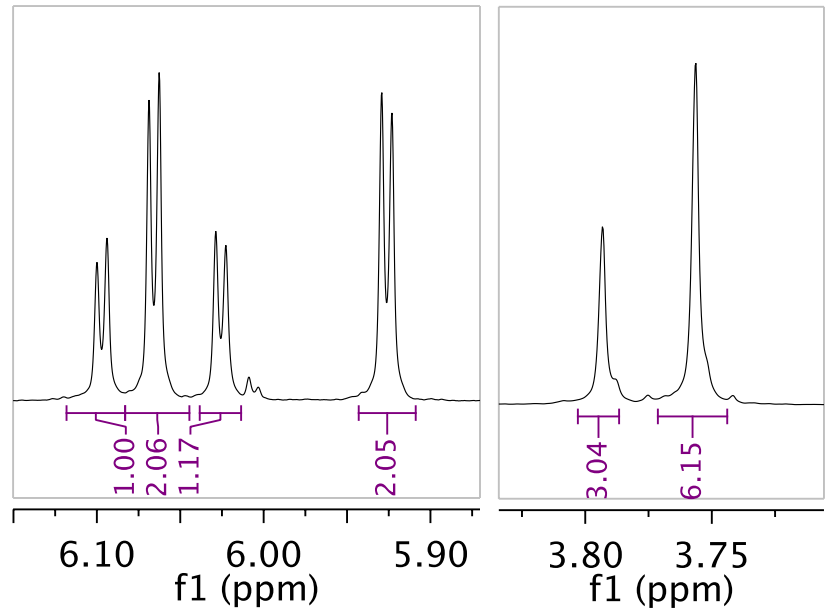

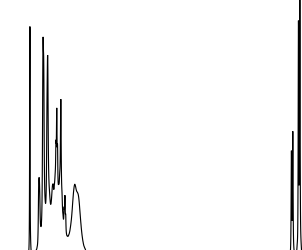
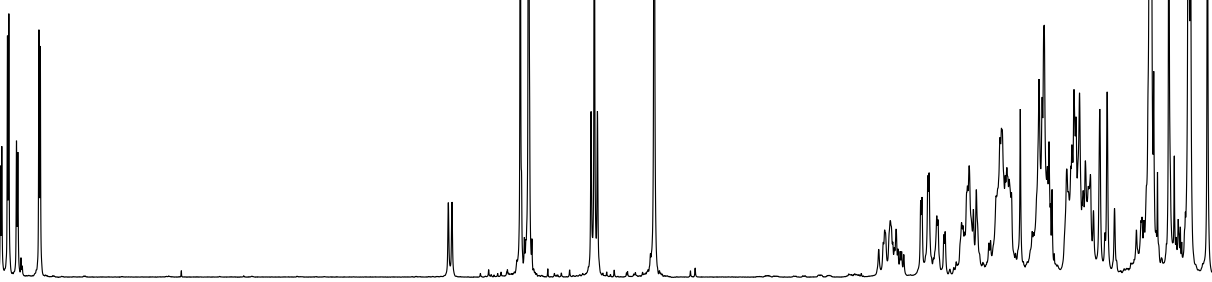

\begin{tabular}{|c|c|c|c|c|c|c|c|c|c|c|c|c|c|c|c|c|c|c|c|c|c|c|c|}
\hline & & & & & & & & $\begin{array}{l}1 \\
\vdots \\
8 \\
\text { in }\end{array}$ & & 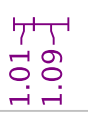 & & & & $\begin{array}{c}T_{1} \\
\tilde{n} \\
0\end{array}$ & $\begin{array}{l}-1 \\
\infty \\
\stackrel{1}{m} \\
m\end{array}$ & $\begin{array}{l}T \\
\top \\
\stackrel{1}{0} \\
\stackrel{-}{-}\end{array}$ & 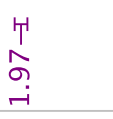 & & 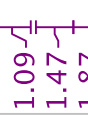 & 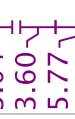 & 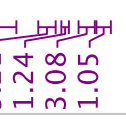 & & \\
\hline 0 & 10.5 & 10.0 & 9.5 & 9.0 & 8.5 & 8.0 & 7.5 & 7.0 & 6.5 & 6.0 & 5.5 & 5.0 & 4.5 & 4.0 & & 3.5 & 3.0 & 2.5 & 2.0 & 1.5 & 1.0 & 0.5 & 0.0 \\
\hline
\end{tabular}




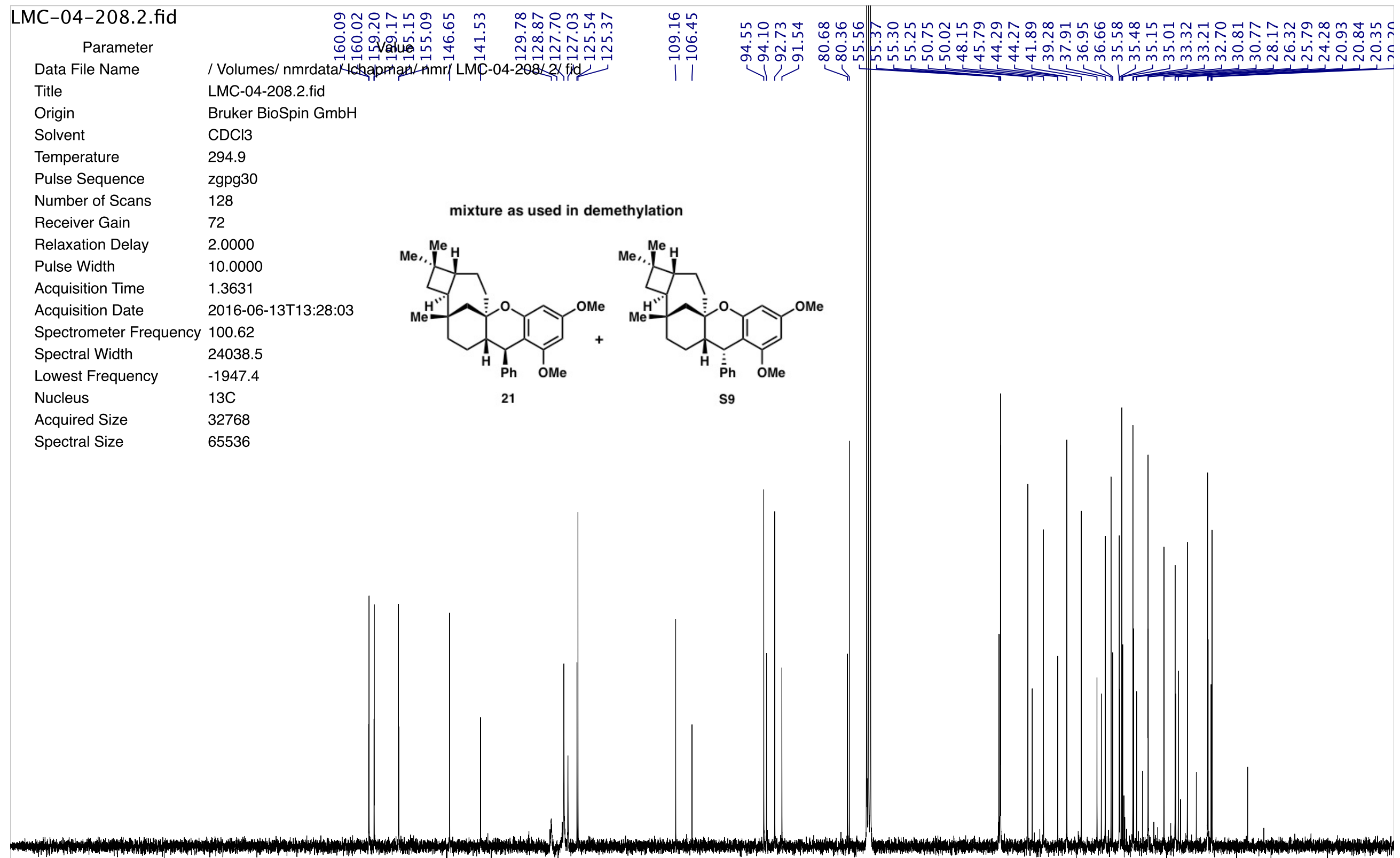

$\begin{array}{llllllllllllllllllllll}210 & 200 & 190 & 180 & 170 & 160 & 150 & 140 & 130 & 120 & \begin{array}{c}110 \\ \mathrm{f} 1(\mathrm{ppm})\end{array} & 90 & 80 & 70 & 60 & 50 & 40 & 30 & 20 & 10 & 0 & -1\end{array}$


LMC-04-213-P1.2.fid

Parameter

Data File Name

Value

/ Volumes/ nmrdata/ lchapman/ nmr/ LMC-04-213-P1/ 2/ fid

Title

LMC-04-213-P1.2.fid

Comment

Origin Bruker BioSpin GmbH

Spectrometer spect

Solvent $\quad \mathrm{CDCl} 3$

Temperature 295.0

Pulse Sequence $\quad \mathrm{zg} 30$

Experiment 1D

Number of Scans 16

Receiver Gain

Relaxation Delay

Pulse Width

Acquisition Time

Acquisition Date

10.0000

11.7000

4.0894

2016-06-30T20:54:19

Spectrometer Frequency 400.13

Spectral Width $\quad 8012.8$

Nucleus $\quad 1 \mathrm{H}$

Acquired Size $\quad 32768$

Spectral Size $\quad 65536$

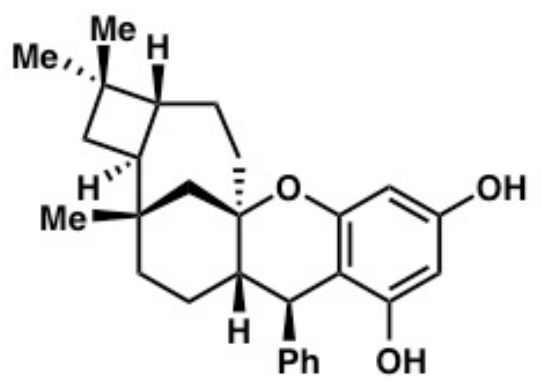

$\mathrm{S} 10$

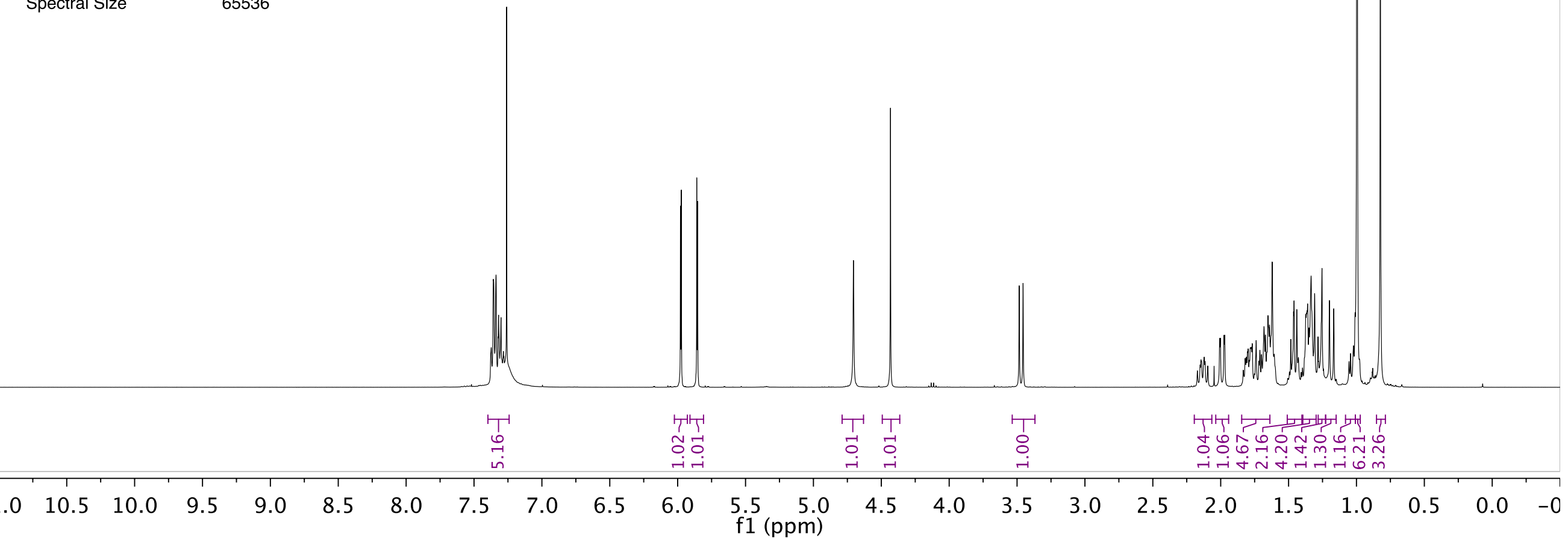




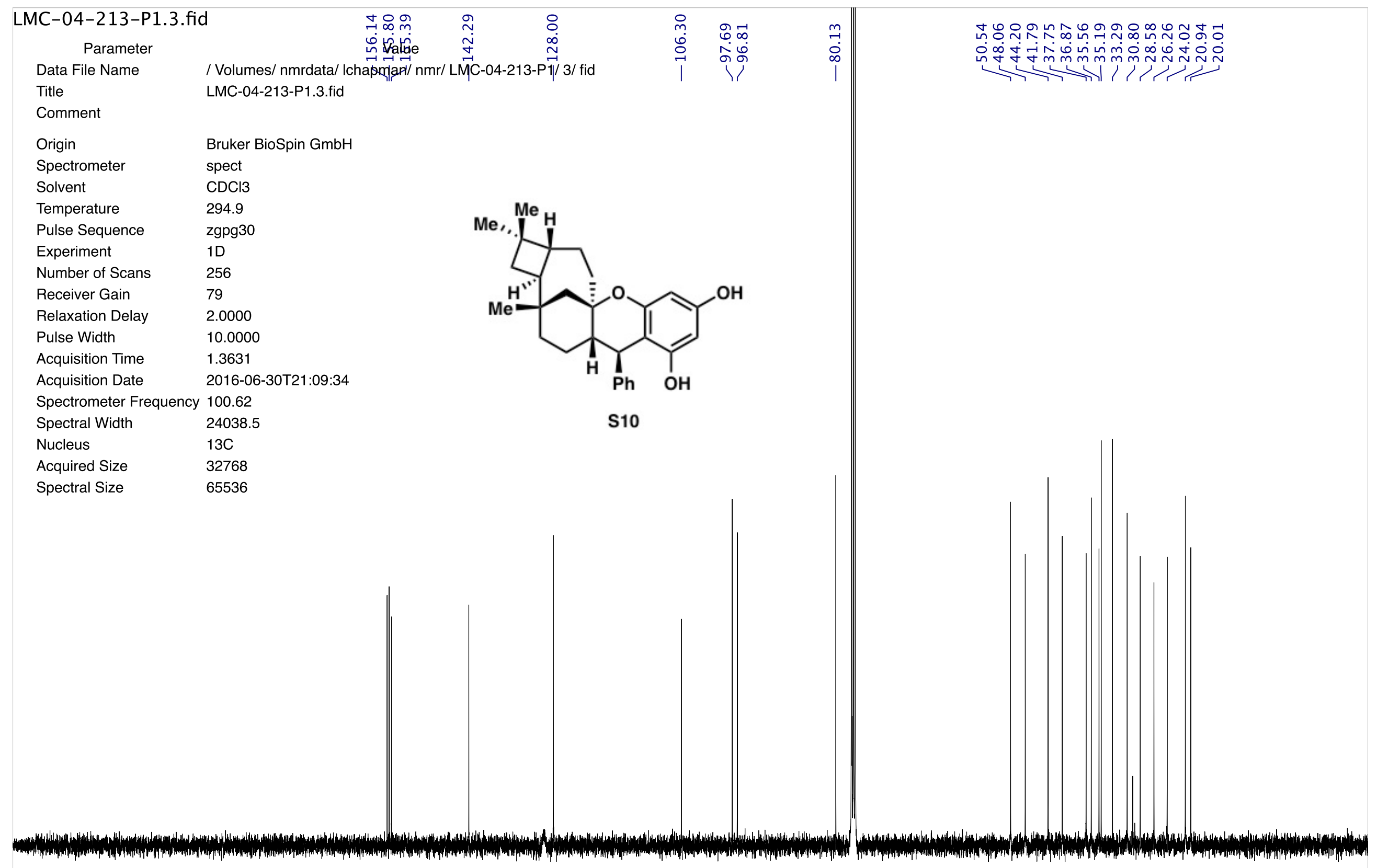

$\begin{array}{lllllllllllllllllllllll}210 & 200 & 190 & 180 & 170 & 160 & 150 & 140 & 130 & 120 & \begin{array}{c}110 \\ \mathrm{f} 1(\mathrm{ppm})\end{array} & 90 & 80 & 70 & 60 & 50 & 40 & 30 & 20 & 10 & 0 & -1\end{array}$


LMC-04-213-P2-flash.1.fid

Parameter

Data File Name

Title

Comment

Origin

Spectrometer

Solvent

Temperature

Pulse Sequence

Experiment

Number of Scans

Receiver Gain

Relaxation Delay

Pulse Width

Acquisition Time

Acquisition Date

Spectrometer Frequ

Spectral Width

Nucleus

Acquired Size

Spectral Size
/ Volumes/ nmrdata/ Ichapman/ nmr/ LMC-04-213-P2-flash/ 1/ fid

LMC-04-213-P2-flash.1.fid

Bruker BioSpin GmbH

spect

$\mathrm{CDCl} 3$

295.0

$\mathrm{zg} 30$

1D

16

156

10.0000

11.7000

4.0894

2016-07-01T14:00:23

400.13

8012.8

$1 \mathrm{H}$

32768

65536

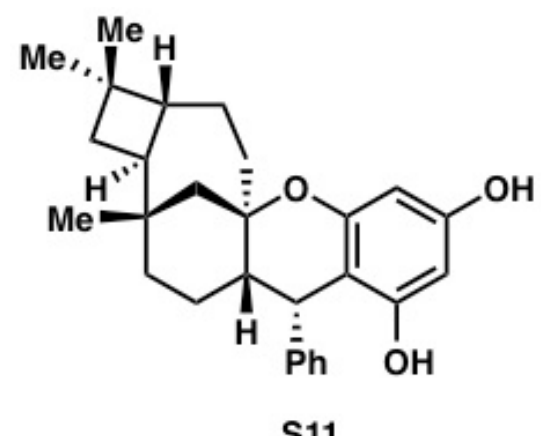

S11

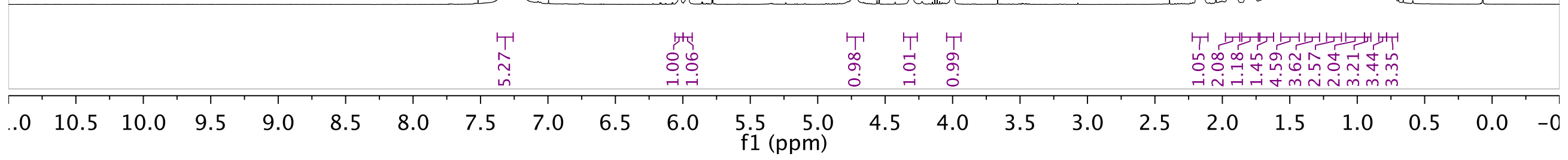




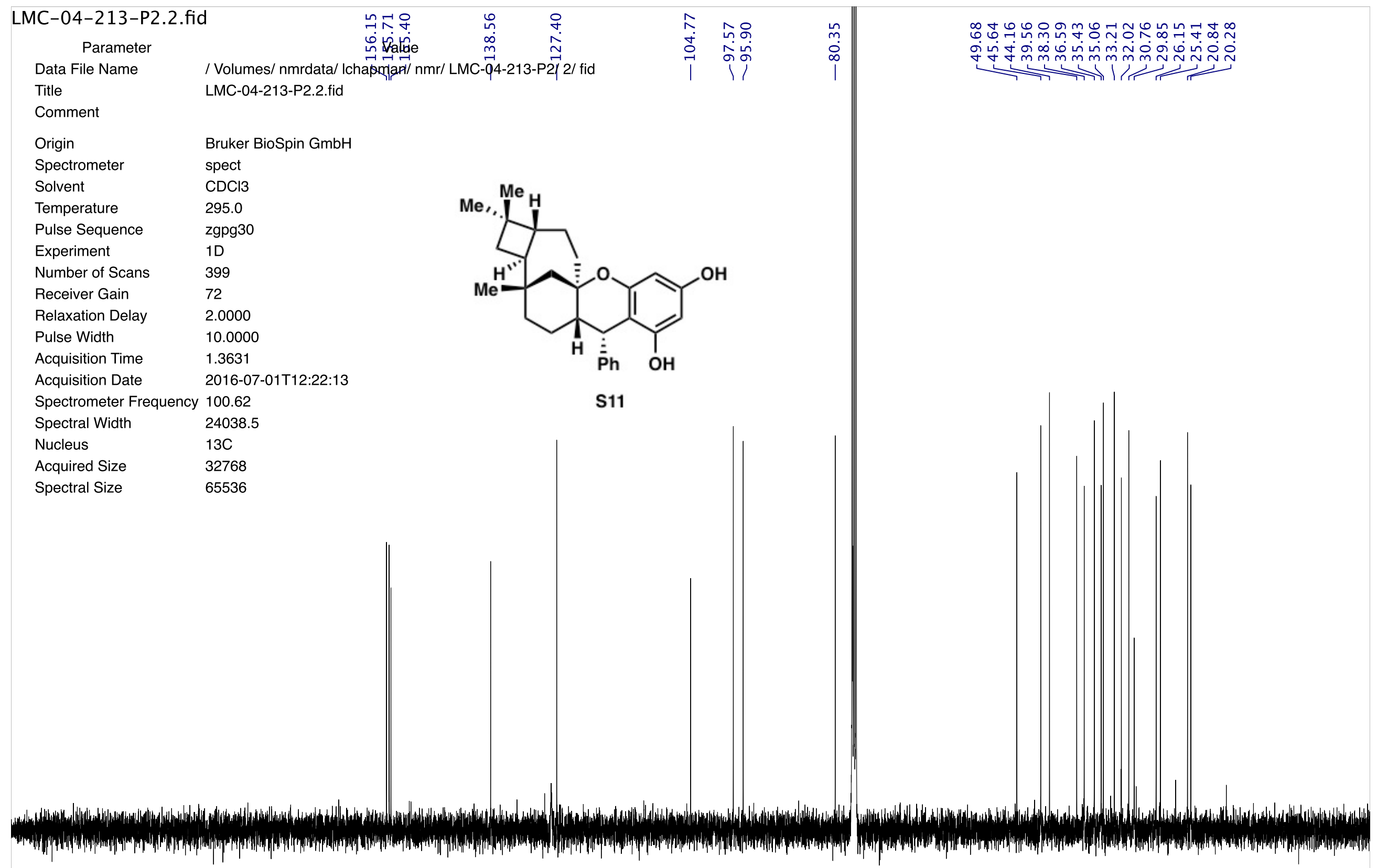

$\begin{array}{lllllllllllllllllllllllll}210 & 200 & 190 & 180 & 170 & 160 & 150 & 140 & 130 & 120 & \underset{\mathrm{f} 1}{110}(\mathrm{ppm}) & 100 & 90 & 80 & 70 & 60 & 50 & 40 & 30 & 20 & 10 & 0 & -1\end{array}$


LMC-04-214-flash.2.fid

\section{Parameter}

Data File Name

Title

Comment

Origin

Spectrometer

Solvent

Temperature

Pulse Sequence

Experiment

Number of Scans

Receiver Gain

Relaxation Delay

Pulse Width

Acquisition Time

Acquisition Date

Spectrometer Frequ

Spectral Width

Nucleus

Acquired Size

Spectral Size
Value

/ Volumes/ nmrdata/ Ichapman/ nmr/ LMC-04-214-flash/ 2/ fid

LMC-04-214-flash.2.fid

Bruker BioSpin GmbH

spect

$\mathrm{CDCl} 3$

295.0

zg30

$1 \mathrm{D}$

16

143

10.0000

11.7000

4.0894

2016-07-02T16:29:45

400.13

8012.8

$1 \mathrm{H}$

32768

65536

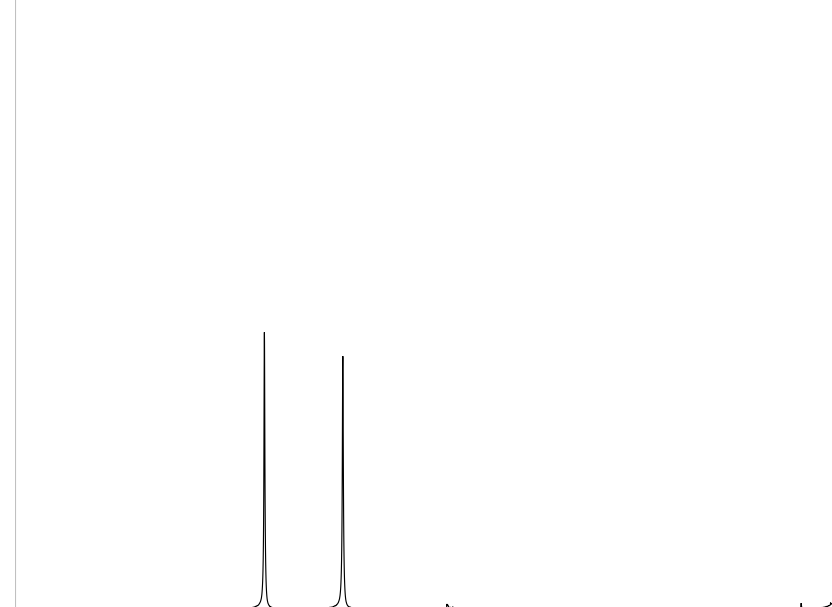

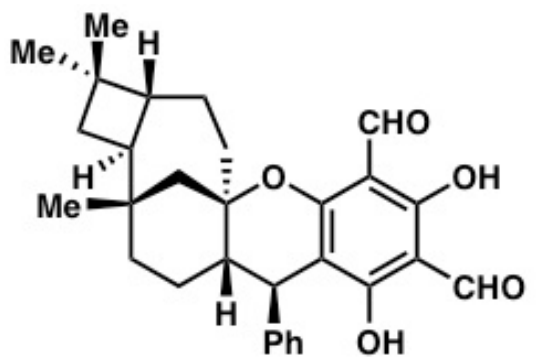

(+)-psiguadial B (1)

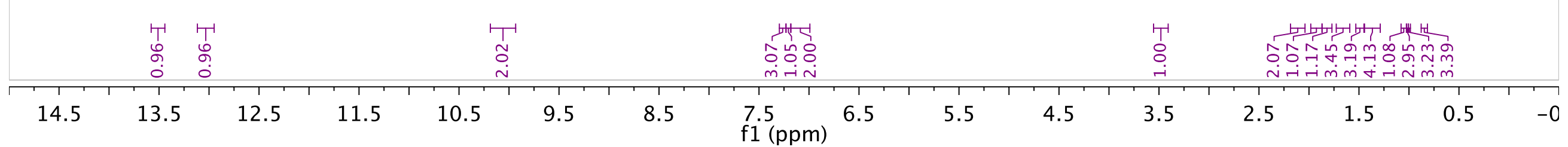




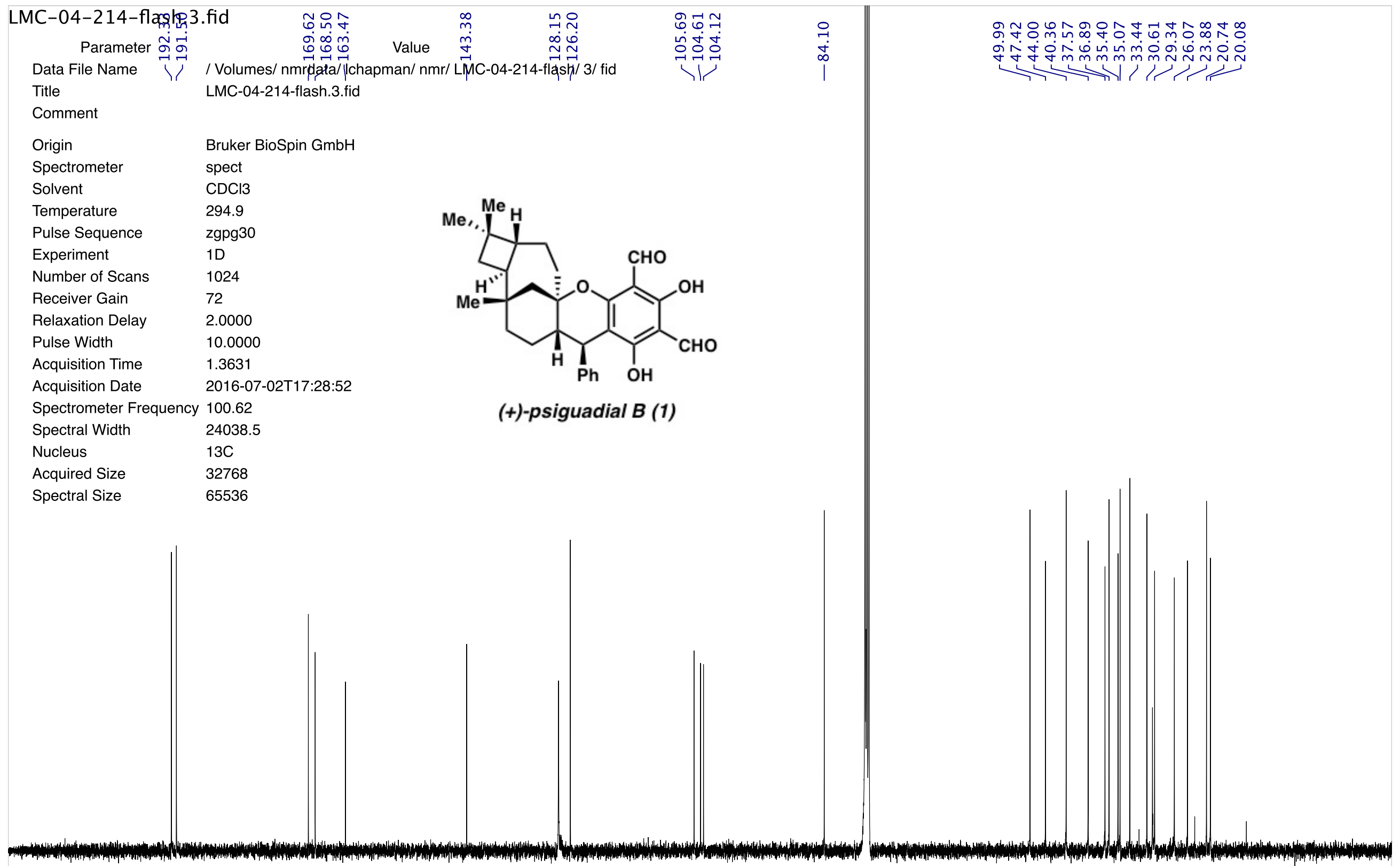

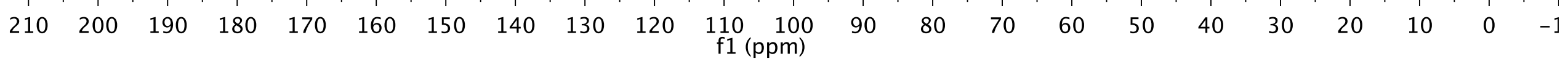

